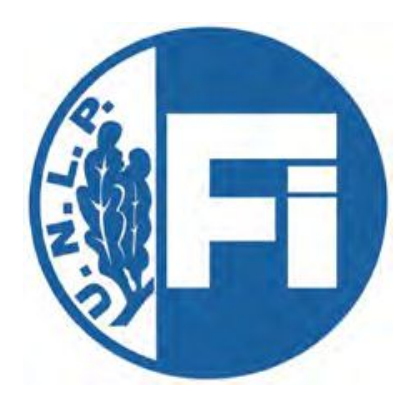

\title{
Nuevas Tecnologías para el Alumbrado Vial
}

Tesis de maestría presentada por Ing. Pablo Ixtaina

ante la Facultad de Ingeniería de la Universidad Nacional de La Plata para optar por el grado académico de

\section{MAGISTER EN INGENIERÍA}

Dirección de Tesis:

Dr. Fausto O. Bredice 


\section{Contenido}

Resumen................................................. 1

Introducción............................................... 2

1. Antecedentes........................................ 4

Cap. 1.- Fundamentos de Luminotecnia...................... 11

1. Nociones de fisiología.............................. 12

2. Magnitudes fotométricas............................ 18

3. Fotometría......................................... 30

Cap. 2.- Alumbrado público y vial.............................. 47

1. Definiciones....................................... 48

2. Evaluación de instalaciones....................... 55

3. Recapitulando................................... 60

Cap. 3.- Caracterización de las propiedades reflectivas de las calzadas...................................... 62

1. Coeficiente de luminancia........................... 63

2. Obtención de la tabla-r............................... 66

3. Aplicación de la clasificación de calzadas.............. 72

4. Sistema de descripción LAL-CIC ...................... 73

Cap. 4.- Medición de las propiedades reflectivas de las calzadas...........................................

1. Medición de los parámetros de descripción............. 77

2. Procedimiento de medición.......................... 82

3. Obtención de la tabla-r.............................. 83

4. Aplicaciones del reflectómetro........................ 84 
Cap. 5.- Evolución de las propiedades reflectivas

de las calzadas.

1. La importancia del estudio............................ 93

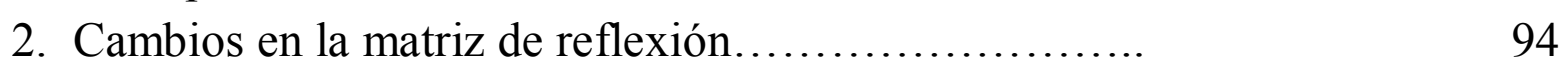

3. Estudios locales................................... 97

4. Medición de calzadas................................. 98

5. Resultados........................................... 100

6. Comentarios finales................................ 102

Cap. 6.- Eficiencia, ahorro de energía y su vínculo con las propiedades de las calzadas............................... 104

1. Caracterización del consumo energético............... 105

2. Eficiencia en instalaciones argentinas................. 110

3. Influencia del pavimento........................... 114

4. Algunas conclusiones.............................. 117

Cap. 7.- Reflexión en calzadas y espectro de la fuente luminosa....................................... 118

1.- Coeficiente de luminancia........................... 119

2.- Grado de claridad bajo iluminación led................ 120

3.- Estudio espectral................................. 124

4.- Conclusiones...................................... 129

Cap. 8.- Análisis ulterior y conclusiones..................... 130

1.- Marco actual: la iluminación LED.................... 131

2.- Producto de la tesis................................. 133

Bibliografía.............................................. 135

Anexo I..................................................... 139 


\section{Resumen}

Esta Tesis desarrolla una propuesta para la mejora de los sistemas de alumbrado vial, tomando como eje la optimización de los parámetros lumínicos de calidad y la eficiencia energética. Para tal fin se realiza el estudio y ajuste del método de cálculo, diseño y evaluación conocido como Técnica de Luminancia. Esta se aplica a sistemas de alumbrado de instalaciones viales y tiene como rasgo central el análisis de la luz reflejada por la superficie de las calzadas hacia el sistema visual del conductor (observador). Su objetivo es predecir cómo refleja la luz la superficie del camino, buscando mensurar un fenómeno complejo, dada la multiplicidad de factores que definen las propiedades ópticas de la superficie de las calzadas: tipo de compuesto asfáltico, material, granulometría, forma de construcción, entre otros.

Se postula que la mejora de los sistemas de alumbrado vial diseñados por la Técnica de Luminancia solo es posible logrando un acabado conocimiento de las propiedades reflectivas del pavimento de la vía de tránsito, que permita incluso la selección de los compuestos asfálticos más favorables para la instalación del alumbrado. Se demuestra que las propiedades reflectivas dependen fuertemente de condiciones locales o regionales, como son las canteras de donde se extrae el material para la construcción de los concretos, la composición del aglutinante o la técnica de construcción. La extrapolación de datos obtenidos en investigaciones realizadas en otros países o zonas (tablas-r) no es posible y dificulta la eficiencia energética. Se analizan, en este sentido, tres aspectos centrales: la reflexión media de la superficie (su "claridad"), el desgaste por el uso y la selectividad espectral (cambio en la reflexión según el color de la fuente luminosa).

Así se demuestra que las calzadas locales son más especulares que las tabla-r estandarizadas; también se desarrolla un método -validado en eventos internacionales- que incluye un equipo de medición para obtener aproximaciones a la tabla-r real a partir de registros en el lugar; y se genera una base de datos con información real de las propiedades reflectivas de las calzadas de la región. 
Introducción 
El objetivo del presente trabajo de Tesis es desarrollar una propuesta para la mejora de los sistemas de alumbrado vial, tomando como eje la optimización de los parámetros lumínicos de calidad y la eficiencia energética. Para tal fin se realiza el estudio y ajuste del método de cálculo, diseño y evaluación conocido como Técnica de Luminancia. Este método se aplica a sistemas de alumbrado de instalaciones viales y tiene como rasgo central el análisis de la luz reflejada por la superficie de las calzadas hacia el sistema visual del conductor (observador). Como se ve en detalle en los capítulos 2 y 3 , el objetivo central de la aplicación de esta técnica es poder predecir cómo refleja la luz la superficie del camino. No obstante, busca mensurar un fenómeno complejo, dada la multiplicidad de factores que definen las propiedades ópticas de la superficie de las calzadas: tipo de compuesto asfáltico, material, granulometría, forma de construcción, por nombrar solo algunos de los aspectos más importantes.

La hipótesis central de esta Tesis puede resumirse de la siguiente manera: la mejora de los sistemas de alumbrado vial diseñados por la Técnica de Luminancia solo será posible logrando un acabado conocimiento de las propiedades reflectivas del pavimento de la vía de tránsito, que permita incluso la selección de los compuestos asfálticos más favorables para la instalación del alumbrado. Se quiere demostrar además, que las propiedades reflectivas dependen fuertemente de condiciones locales o regionales, como son las canteras de donde se extrae el material para la construcción de los concretos, la composición del aglutinante o la técnica de construcción; todos ellos, factores que tornan difícil la extrapolación de datos obtenidos en investigaciones realizadas en otros países o zonas. Finalmente, la hipótesis se completa con tres aspectos que, según se sostiene, afectan y determinan el desempeño de la instalación tanto desde el punto de vista perceptivo como del energético: reflexión media de la superficie (su "claridad"), desgaste por el uso y selectividad espectral (cambio en la reflexión según el color de la fuente luminosa).

El desarrollo de esta Tesis se basa principalmente en resultados de mediciones de iluminación, experiencias y datos relevados en autopistas de la región: de acceso a la Ciudad de Buenos Aires (CABA) (acceso Norte, con sus ramales Tigre, Pilar y Campana; Avenida General Paz; acceso Oeste; corredor Ezeiza-Cañuelas y La Plata-Buenos Aires) y las autopistas urbanas de CABA: 25 de Mayo, Perito Moreno, Illia y 9 de Julio Sur. En este punto debe mencionarse que el acceso a las vías de tránsito para realizar experiencias constituyó una situación compleja, dado que se debió obtener la autorización del concesionario o responsable del corredor vial, coordinar y lograr el permiso del sector Seguridad Vial, respetar zonas horarias con menor carga de tránsito (por lo general entre 1:00 am y 4:00 am) y cumplir con los esquemas de cortes regulados por el contralor (gobierno de CABA, Vialidad Nacional, Vialidades provinciales). En este sentido, resultó imposible la liberación total de la traza y fueron limitados los carriles que pudieron cortarse simultáneamente para efectuar pruebas (por lo general no más de dos).

Dadas estas condiciones, gran parte de la investigación pudo realizarse gracias a un convenio firmado entre la Comisión de Investigaciones Científicas (CIC) y el Órgano de Control de Concesiones Viales (OCCOVI), hoy absorbido por Vialidad Nacional. Este acuerdo, cuya versión original data del año 2000, tiene como objeto realizar evaluaciones de luminancia e iluminancia sobre las calzadas de las autopistas concesionadas a razón de 20 a 30 áreas evaluadas por año y por acceso; viene siendo 
instrumentado por el Laboratorio de Acústica y Luminotecnia (LAL), Centro de Investigación propio de la Comisión de Investigaciones Científicas (CIC), ${ }^{1}$ que está a cargo de las dichas evaluaciones. Asimismo, y con similar esquema, se efectuaron transferencias tecnológicas con la empresa Autopistas Urbanas S.A. (AUSA) (a cargo de las autopistas dentro de la ciudad de Buenos Aires), con Vialidad de la provincia de San Luis (estudios realizados en el tramo iluminado de la RN 7), Vialidad de Córdoba (avenida de circunvalación) y la empresa Puentes del Litoral (a cargo originariamente del corredor Rosario - Victoria). Como se comprende, los análisis, que permitieron realizar investigaciones sobre trazados de autopistas habilitadas al tránsito, debieron adecuarse al ritmo de estas demandas que, si bien ofrecieron una posibilidad casi única de acceso al objeto de estudio, establecieron un regularidad tal que explicó la duración de la investigación (más de 15 años) y la necesidad de establecer etapas que se adecuaran a los distintos estadios de las instalaciones: evaluaciones y pruebas sobre la obra nueva, mediciones con la instalación depreciada, recambios, testeos de nuevos artefactos, hasta la etapa actual de reconversión a tecnología LED.

El autor de la presente Tesis, investigador CIC, participó directamente de las actividades mencionadas realizadas por el LAL. Primeramente, se desempeñó como miembro del personal científico de la institución y, desde 2012, como director del LAL y está a cargo de los equipos de medición que trabajan en las autopistas. La totalidad de los estudios aquí presentados forma parte de los trabajos realizados en el marco de los Planes de Investigación oportunamente presentados y evaluados por el Directorio de la CIC. Se han extraído avances de Informes Científicos y publicaciones internas del $\mathrm{LAL},{ }^{2}$ así como de presentaciones en congresos y publicaciones científicas del autor. Parte del material se corresponde también con informes tecnológicos elaborados a organismos o empresas contratantes, lo que impone políticas de reserva enmarcadas en el sistema de Calidad del LAL. Por estos motivos, se han eliminado los vínculos o referencias directas a resultados de mediciones, mencionándose solamente parámetros globales y limitando la identificación cuando se entendió que afectaba intereses de los contratantes.

\section{1.- Antecedentes}

\section{1.- El Alumbrado Vial y la Técnica de Luminancia}

El objetivo de un sistema de iluminación artificial para alumbrado vial es proporcionar las condiciones visuales necesarias para un tránsito seguro y confortable, con un mínimo costo total de la instalación del alumbrado. Los estudios de la percepción han demostrado que los mejores escenarios se logran cuando los obstáculos son vistos por los conductores como siluetas oscuras contra un fondo

\footnotetext{
${ }^{1}$ Laboratorio de Acústica y Luminotecnia - Comisión de investigaciones Científicas de la provincia de Buenos Aires, [en línea] URL: http://lal.cic.gba.gob.ar/ [Consulta: 2 de abril de 2019].

2 "Informes Científicos del Investigador", Repositorio Digital CIC, [en línea] URL: https://digital.cic.gba.gob.ar/discover?query=informes + cient $\%$ C3\%ADficos\&lr $=$ [Consulta: 2 de abril de 2019].

"Memorias del LAL. Memorias CIC PBA", Repositorio Digital CIC, [en línea] URL: https://digital.cic.gba.gob.ar/handle/11746/1812 [Consulta: 2 de abril de 2019].
} 
más brillante, que es la propia calzada. ${ }^{3}$ Aquí surge el concepto central de la Técnica de Luminancia: el contraste se cuantifica a partir de la diferencia entre la luz reflejada por la calzada, que ingresa al sistema visual del conductor, y la reflejada por el objeto a detectar. Dado que esta diferencia se reproduce directamente en la retina del ojo, es posible lograr una buena aproximación a la percepción visual (puramente subjetiva) e intentar cuantificarla. Si bien este tema se verá con más detalle en el capítulo 1, aquí se anticipa que, en base a esta relación entre luminancia y percepción, es factible regular la cantidad de luz sobre la calzada de modo de llegar a lo justo y necesario para lograr un tránsito seguro con un mínimo consumo de energía.

La puesta en práctica de estos del concepto de la Técnica de Luminancia, a partir del modelado de la instalación vial, el estudio de la percepción del observador desde la posición de conductor y, principalmente, el fenómeno de reflexión en la calzada, fueron objeto de investigación desde mitad del siglo pasado. ${ }^{4}$ A mediados de los '70, los estudios convergieron hacia la técnica de aplicación que aún se mantiene vigente. Además del trabajo de De Boer, Erbay primero y Sorensen unos meses después, publicaron sendas recopilaciones de la técnica de aplicación y los muy importantes "Atlas de Pavimentos". 5 Unos años después, la Comisión Internacional de Alumbrado CIE estandarizó la técnica a partir de estas investigaciones en su recomendación $30 .^{6}$

Los "Atlas de Pavimentos" consistieron en recopilaciones de mediciones estandarizadas de muestras de pavimentos, cuyas características reflectivas -como se verá en el capítulo 3- pueden representarse por una matriz numérica. Erbay reunió una colección de 240 superficies medidas por la Universidad de Berlín, obtenidas de varias regiones de Alemania. Sorensen, por su parte, realizó un trabajo similar en pavimentos daneses y suecos. En función de las necesidades de su región (clima con una alta tasa anual de días lluviosos o con condiciones de calzada húmeda), este segundo autor incorporó las situaciones experimentales de humedad en sus mediciones de muestras de calzada. Así logro reunir 347 matrices, clasificando pavimentos secos y mojados según las condiciones estandarizadas para esa fecha. La importancia de estas recopilaciones radicó en que por primera vez se pudo tener una idea acabada de la extrema dispersión de las formas de reflexión. Se encontraron desde superficies "difusas" (más adelante definiremos cuantitativamente el concepto de "difuso", pero podríamos imaginarlo como la superficie de un hormigón claro) hasta otras con direcciones de reflexión muy selectivas. Estas últimas, por la tendencia a reflejar en forma similar a un espejo, se denominan "superficies especulares". Tanto Erbay como Sorensen presentaron sus resultados ordenando sus matrices desde las más difusas hacia las más especulares, tratando de mantener un orden creciente de selectividad de la reflexión. Esta idea de "ordenar" presupone algún número único o combinación de

\footnotetext{
3 "Memorias del LAL. Memorias CIC PBA", op. cit.; Blackwell H. R., "Contrast thresholds of the human eye". Journal of the Optical Society of America, 36(11), 1946.

${ }^{4}$ Ver, por ejemplo: Blackwell H. R., "Contrast thresholds...”, op. cit.; De Boer, J., Cohu, M., Schreuder, D., Public Lighting. Philips technical Library, Países Bajos, 1967; De Boer J., Burghout F., Van Heemskerck Veeckens, J. F. T., "Appraisal of the quality of public lighting based on road surface luminance and glare", 14va Sesión del CIE, Bruselas, P59-23, 1959.

${ }^{5}$ Erbay A., Der Reflexionseigenschaften von Fahrbahndecken. University of Technology, Berlin, 1974; Sørensen K., Road Surface reflection data. Lysteknisk Laboratorium, The Danish illuminating Engineering Laboratory. Lyngby, Dinamarca, Reporte $\mathrm{N}^{\circ} 10,1975$.

${ }^{6} \mathrm{CIE}$, Calculation and measurement of luminance and illuminance in road lighting, Publicación 30, 1976.
} 
índices que permiten detectar dentro de los aproximadamente 400 coeficientes que componen cada tabla de reflexión, su grado de especularidad. Como veremos posteriormente, esto no es simple y tampoco lo es la comparación de superficies entre sí.

En nuestro país, los trabajos con la Técnica de Luminancia se iniciaron entre 1978 (iluminación de la Avenida del Libertador, en Vicente López) y 1980 (autopista urbana 25 de Mayo). Las empresas dominantes en el mercado local de iluminación (Philips, Siemens y Strand) tenían un vínculo europeo importante, y como vimos, justamente allí se estaban desarrollando en ese momento, las investigaciones sobre el tema. De este modo y a pesar de que en nuestro país no había aún una norma local sobre alumbrado público, los pliegos de licitación hicieron referencia a valores de luminancia. Fue el Área Luminotecnia del Laboratorio de Entrenamiento Multidisciplinario para la Investigación Tecnológica (LEMIT), ${ }^{7}$ que posteriormente derivaría en el LAL, el encargado de realizar las mediciones de campo, sin antecedentes en la región. Lamentablemente, quedan escasos registros escritos de esos trabajos; solo se recupera el antecedente, rescatado de informes de medición, de valores de luminancias puntuales medidos con discrepancias con las luminancias de cálculo y proyecto. Debe remarcarse que, en ese tiempo, los cálculos se efectuaban por métodos gráficos, sin la ayuda de ordenadores y por ende eran limitados. Además, se desconocían las propiedades reflectivas de los pavimentos locales, usándose en su lugar matrices de reflexión seleccionadas de los estudios europeos.

Las discrepancias encontradas en estas primeras evaluaciones, entre valores de luminancia de proyecto y los resultados de la medición en el lugar, dieron origen a una línea de investigación que tuvo como eje tratar de medir muestras de calzadas locales. El trabajo fue realizado justamente por el Área Luminotecnia del LEMIT, que estaba consolidando ya los grupos de investigación del recientemente fundado LAL. Los investigadores trataron, por un lado, de construir un equipo para evaluación de muestras a partir del uso de un luminancímetro según la propuesta de Pritchard ${ }^{8}$ (se verán detalles de tales instrumentos en el capítulo 1 y 2). El otro objetivo fue ajustar los métodos de extracción para llegar a probetas de tamaño estandarizado, minimizando las alteraciones en la superficie debidas a los cortes necesarios para retirarlas de las calzadas. Los primeros resultados de esta investigación, cuya conclusión central fue que los pavimentos estudiados (provenientes de rutas de la provincia de Buenos Aires) eran muy distintos en cuanto a sus propiedades reflectivas de los publicados en la bibliografía europea, fueron expuestos en las $V$ Jornadas Argentinas de Luminotecnia, en Rosario (1982). ${ }^{9}$

\footnotetext{
${ }^{7}$ Laboratorio de Entrenamiento Multidisciplinario para la Investigación Tecnológica (LEMIT), [en línea] URL: http://www.lemit.gov.ar/ [Consulta: 2 de abril de 2019].

8 "Company", Photo Research. A Line of Light \& Color Measurement Soutions, [en línea] URL: http://www.photoresearch.com/content/company [Consulta: 2 de abril de 2019].

${ }^{9}$ Alcalde, H., Bazalar Vidal, P., "Investigación de pavimentos de la provincia de Buenos Aires", Memorias de las $V$ Jornadas Argentinas de Iluminación, Rosario, 1982.
} 

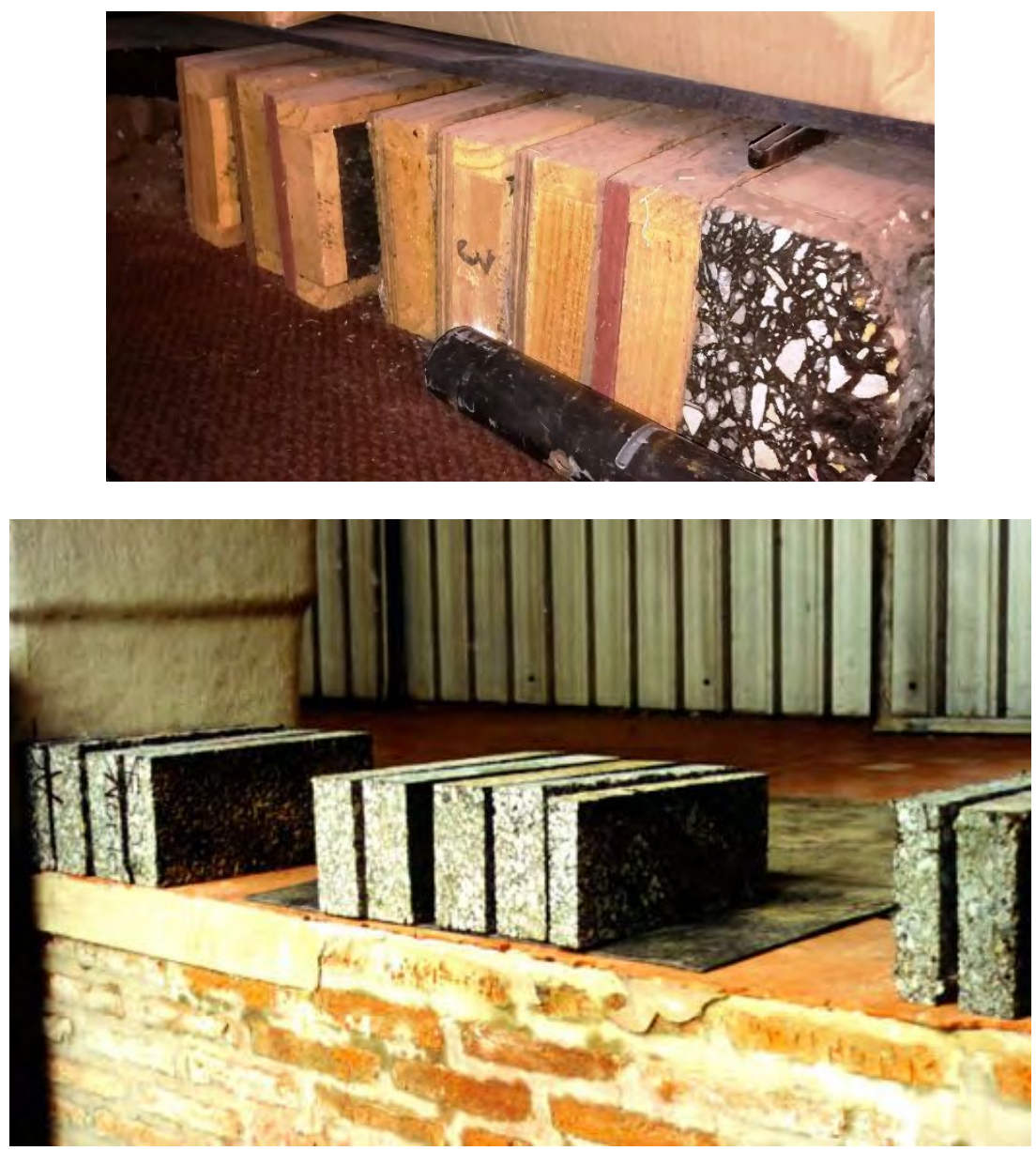

Figuras 1 y 2. Muestras de calzadas acondicionadas para medir (fotos del archivo del LAL).

En el marco del estudio citado, se perfeccionó la medición de muestras. También se evidenciaron las diferencias en cuanto a la reflexión que introducía el uso (carga de tránsito) y la intemperie, frente a una calzada recién construida. Esto motivó el desarrollo de un método experimental de pre acondicionamiento de las muestras, para simular los primeros meses de uso, y el diseño de un equipamiento especial. 


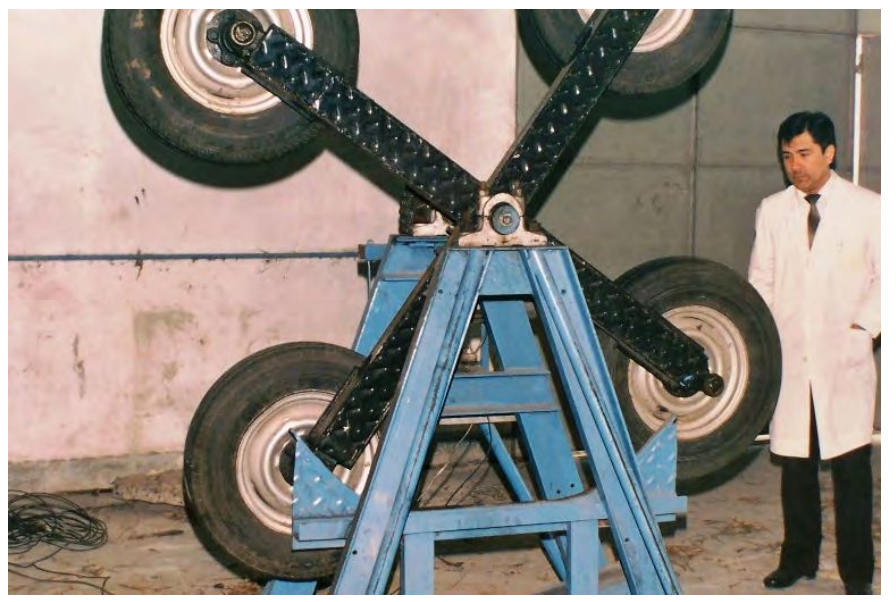

Figura 3. Equipo experimental para el desgaste de probetas diseñado y construido en el LAL (foto del archivo del LAL).

\section{2.- El uso de pavimentos “drenantes" y la formulación de las hipótesis de trabajo}

Los diseños de instalaciones de alumbrado por la Técnica de Luminancia en el país se interrumpieron a mediados de los ' 80 , luego de la construcción de las autopistas urbanas de la ciudad de Buenos Aires. Las pruebas de campo se limitaron y las investigaciones se centraron en estudios teóricos y en el análisis de datos basados en mediciones ya realizadas. Recién para fines de los ' 90 , con las obras de ampliación y remodelación de los principales corredores de acceso a CABA (Acceso Norte, Oeste y posteriormente Ezeiza - Cañuelas), se pudo volver a tener acceso a autopistas iluminadas y continuar con las investigaciones experimentales. El autor de la presente Tesis se incorpora y posteriormente dirige los estudios de esta nueva etapa, que tuvo como rasgo significativo la aparición de las calzadas del tipo "drenante" que poseían una granulometría y aglutinante tal que generaron matrices de reflexión muy distintas a las de la década previa. Estos pavimentos agudizaron las discrepancias entre proyecto y evaluación de la instalación y, fundamentalmente, comenzaron a evidenciar que nuestras calzadas no eran asimilables a los estándares europeos de reflexión citados por la bibliografía internacional. ${ }^{10}$

\footnotetext{
${ }^{10}$ CIE, Road surface and road marking reflection characteristics, publicación TC 4.24 y 4.25-144, Francia, 2001.
} 


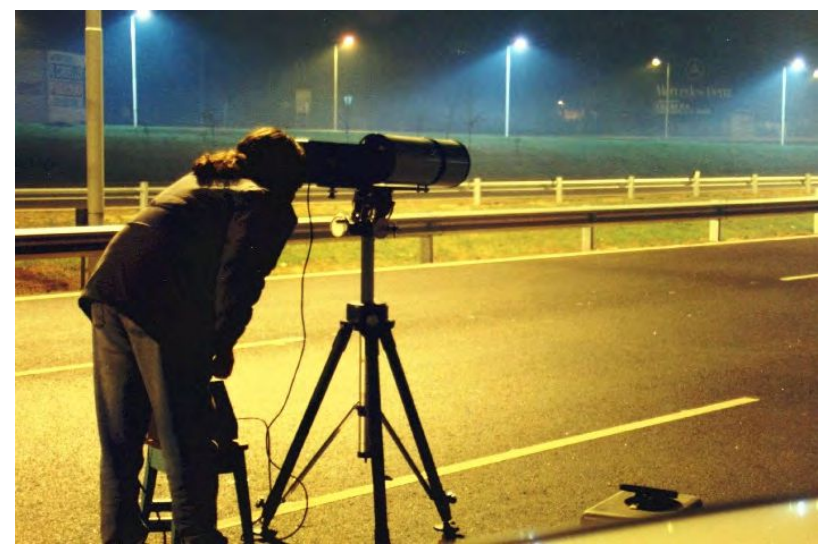

Figura 4. Imagen de una medición de luminancia en calzada realizada por el autor (1998, Au. del Oeste -Foto del autor-).

Entre los rasgos característicos de esta etapa se encuentra el elevado número de instalaciones nuevas que no verificaban los valores proyecto, ajustados a lo normalizado por IRAM para la clase de vía de tránsito calificada como "autopistas". ${ }^{11}$ Atendiendo a estas discrepancias y tratando de mejorar los proyectos, los concesionarios viales junto con los encargados de la obra de iluminación realizaron numerosas consultas al laboratorio que dieron impulso a la línea de investigación vinculada con la Técnica de Luminancia, generando nuevas transferencias de tecnología. Sobre esta base comenzó la definición de las hipótesis de trabajo que guiaron los Planes de Investigación CIC del autor y que finalmente se plasmaron en la presente Tesis, a saber:

a.- Las propiedades reflectivas de los pavimentos de nuestra región no pueden asimilarse a los estándares europeos. La correcta aplicación de la Técnica de Luminancia requiere entonces de un método práctico para la caracterización de las carpetas asfálticas.

El desarrollo de esta hipótesis se inicia en el capítulo 2, en donde se resumen los conceptos generales del alumbrado vial, lo central de la Técnica de Luminancia y las prácticas recomendadas. En el capítulo 3, y sobre la base de trabajos de investigación originales del autor, se verifica por varios caminos, lo central de la hipótesis. Finalmente, y a modo de resultado innovador de este estudio, se describe el método y equipo diseñado por el grupo de investigación que dirige el autor, para la caracterización (medición) de calzadas. Este instrumento original fue presentado en el Simposio International de la Comisión Internacional de Alumbrado CIE: Symposium on road surface photometric characteristics: Measurement Systems and Results, que tuvo lugar Torino, Italia, en julio de 2008. En este evento, último organizado por la CIE específicamente sobre el tema de caracterización lumínica de calzadas, el autor expuso los detalles del equipo, su construcción, calibración y experiencias de aplicaciones concretas realizadas en autopistas de la zona. Se verificó además que, si bien existían un par de propuestas de instrumental experimental para el mismo fin, nuestro desarrollo era el único

\footnotetext{
${ }^{11}$ Instituto Argentino de Racionalización de Materiales, IRAM AADL J 2022-2, Alumbrado Público, Vías de Tránsito Clasificación y Niveles de Iluminación, Buenos Aires, 1995.
} 
implementado y con verificaciones y experiencias reales de uso en autopistas. Los detalles de este estudio se dan en el capítulo 3.

La hipótesis se completa con el análisis de los siguientes aspectos:

b.- Las propiedades reflectivas del pavimento no pueden considerarse una constante, por el contrario, evolucionan con el uso y el tiempo de instalación de la carpeta, hasta llegar a un estado estacionario. En el capítulo 4 se desarrolla y se demuestra este postulado, a partir del seguimiento del desempeño de instalaciones de alumbrado, sin cambios de superficie o recapados, por largos períodos de tiempo.

c.- Existe un vínculo directo entre reflexión en calzada y la eficiencia energética lograda en la instalación de alumbrado, punto desarrollado en el capítulo 5, en donde se demuestra la posibilidad cierta de mejorar sustancialmente la eficiencia energética mediante la selección adecuada de compuestos asfálticos.

d.- Existe un vínculo entre el espectro de la fuente luminosa y la reflexión, tema analizado en el capítulo 6, mediante la comparación fotométrica y espectral de lo que hoy podemos considerar las fuentes tradicionales (lámparas de sodio alta presión) y las nuevas tecnologías, principalmente luminarias LED. 
Capítulo 1 - Fundamentos de luminotecnia 
Cómo se anticipó, el núcleo de esta Tesis es la mejora de los sistemas de alumbrado vial, tomando como eje la optimización de los parámetros lumínicos de calidad y la eficiencia energética. Para tal fin se propone el estudio y desarrollo de la Técnica de Luminancia, orientado a la caracterización de las propiedades reflectivas de los compuestos asfálticos y su interacción con las fuentes luminosas de nueva tecnología (LEDs). La mencionada técnica forma parte de la luminotecnia, entendida ésta como un conjunto de prácticas cuyo objetivo es la producción, adecuación y uso eficiente de la iluminación artificial, su control y aplicación para fines específicos.

En este sentido, se considera central iniciar con una presentación de la luminotecnia y un recorrido por sus fundamentos, comenzando por los modos en que impacta la luz en los individuos, a través de las nociones de fisiología de la visión, sensibilidad espectral y percepción del color. Posteriormente, se referirá a las llamadas magnitudes fotométricas, forma específica de caracterizar la radiación electromagnética que se conoce como luz, de modo de describir los fenómenos que hacen a la luminotecnia: su cuantificación, el vínculo con la percepción, las relaciones fundamentales y los cálculos básicos. Finalmente, se efectuará una introducción al campo de la medición de la luz: la llamada fotometría.

\section{1.- Nociones de fisiología de la visión, sensibilidad espectral y color}

En el proceso de visión intervienen dos conceptos que conviene definir y diferenciar como primer paso en el estudio: ${ }^{1}$

- Un estímulo luminoso.

- La sensación o percepción visual.

El estímulo luminoso, o en forma más simple la luz, rige en gran medida todas las actividades humanas. El sol es la fuente principal de luz, aunque no debe sorprender que desde tiempos remotos el hombre haya intentado producirla por medios "artificiales": fuego, antorchas, velas, lámparas eléctricas.

Resulta posible ensayar una definición de luz, desde el punto de vista de la tecnología de la iluminación, como la energía radiante que puede evaluarse visualmente, en otras palabras, aquella energía que produce percepción visual. Acercándonos más a la física, encontramos que la luz (radiación visible) es una porción del espectro electromagnético, con longitudes de onda limitadas entre aproximadamente 380 y 760 nanómetros [nm]. Las ondas electromagnéticas, con sus propias frecuencias reflejadas o emitidas por los diversos objetos, activan el sistema visual humano y los tornan visibles.

Definida en términos de radiación electromagnética, la luz puede ser convenientemente medida. En función de los intereses aquí planteados, se habla de intensidad luminosa, flujo, densidad de flujo, dirección de los rayos luminosos, etc., todos parámetros posibles de medir o calcular en forma más o menos exacta. En este sentido, se afirma, por ejemplo, que la cantidad de luz sobre una determinada superficie es de $x$ lux, aseveración ésta que puede ser verificada por diversos observadores, siempre que sus herramientas de cálculo y medición sean las adecuadas.

\footnotetext{
${ }^{1}$ Moon, P., The Scientific Basis of Illuminating Engineering, McGraw Hill Book Company, Londres, 1936; Bardier, D., De la Visión al Conocimiento, Tradinco editores, Montevideo, 2001.
} 
No sucede lo mismo con la percepción visual, el otro concepto involucrado en la visión. Ésta es cien por ciento subjetiva; no hay manera de registrarla o medirla, más allá de la sensación de las propias personas. Tomando como base que el lenguaje es el único medio que posee un individuo para comunicar sus experiencias visuales, éste impondrá el límite de conocimiento al que podrá llegar un observador externo. Cuando se exploran lo limites o umbrales de percepción, la subjetividad se pone de manifiesto: la escena claramente visible para unos sujetos, se torna apenas discernible para otros; las expresiones de "nivel cómodo y agradable", "confortable", "justo lo necesario", aparecen, en términos de estímulo luminoso, como variables con muy alto rango de incertidumbre para su definición.

Podemos concluir este primer punto afirmando que no existe una relación lineal y biunívoca entre estímulo luminoso y percepción visual. Sí advertimos aproximaciones que, en determinadas circunstancias, permiten asegurar a qué nivel de iluminación una tarea visual podrá ser realizada correctamente por un muy alto número de personas. Para conocer más sobre los límites de tales planteos, es que se torna necesario introducirnos al sistema visual humano.

\section{1.- Fisiología de la visión}

Para que podamos percibir una imagen, los rayos luminosos que penetran en el ojo deben ser enfocados sobre su pared posterior, que se encuentra recubierta por una delicada membrana llamada retina. El ojo se comporta como una "cámara oscura", dado que la luz es refractada por la lente (el "cristalino") y proyecta una imagen nítida sobre la pared posterior.

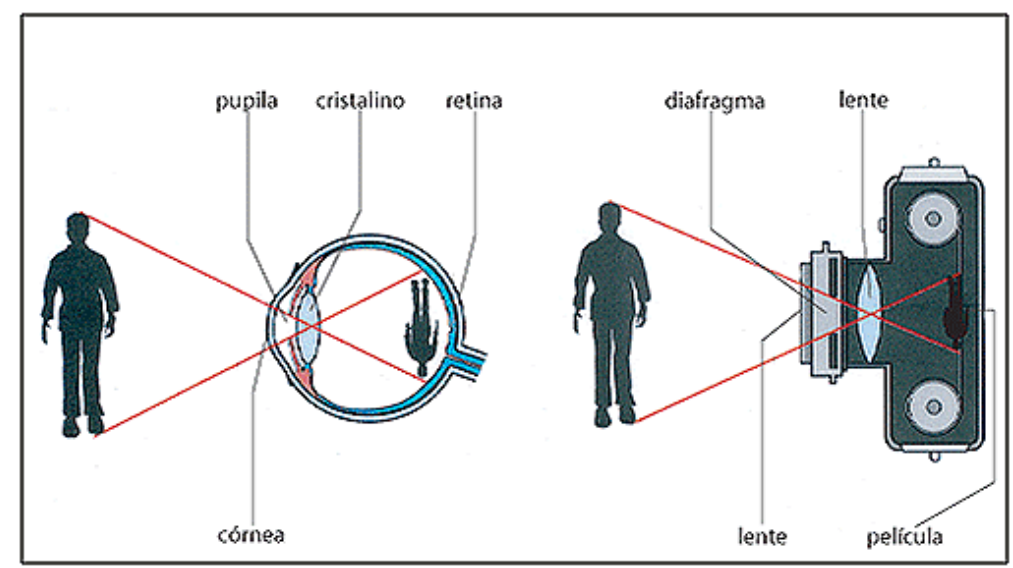

Figura 5. Formación de imágenes en el ojo. ${ }^{2}$

En la retina existen dos tipos de células especializadas, sensibles a los rayos luminosos: los conos y los bastones. En ellas, la incidencia de luz induce reacciones químicas generadoras de estímulos nerviosos. En el mismo espacio, también actúan otros grupos de células nerviosas, que se encargan de transmitir los impulsos originados por los conos y los bastones hacia el nervio óptico, el cual abandona la región posterior del globo ocular un poco hacia el lado nasal de su centro.

\footnotetext{
2 Imagen tomada de: Narváez, A., "La microscopía", Nociones básicas de óptica (capítulo 2), [en línea] URL: http://www.medic.ula.ve/histologia/anexos/microscopweb/ MONOWEB/capitulo2 5.htm [Consulta: 9/4/19].
} 
La imagen que corresponde a la línea central de visión se forma en la zona foveal de la retina. Allí es descompuesta en decenas de miles de impulsos nerviosos por los conos que, en dicha región, se encuentran altamente concentrados. El área cubierta se conoce como campo detallado de visión, que es la pequeña porción que queda definida cuando el observador "fija" la vista dentro de una escena visual. Es un cono realmente estrecho que cubre un ángulo de alrededor de un minuto (aproximadamente la superficie de uno de los pulgares visto a la distancia del brazo extendido). Se tiene sobre esta zona visión de detalle con diferenciación de colores. ${ }^{3}$

El campo detallado es interior a otro cono más amplio, que es el campo general o periférico de visión. Éste es un cono irregular, que alcanza en términos medios $30^{\circ}$ hacia arriba, $45^{\circ}$ hacia abajo y $65^{\circ}$ a ambos lados. La forma del rostro humano enmarca estos límites. La percepción en este cono está dada por los estímulos generados principalmente por bastones y los conos situados fuera de la zona foveal. Como la concentración de éstos disminuye rápidamente en la medida en que se aleja hacia la periferia de la retina, la visión que se obtiene en la zona periférica será de siluetas o de conjunto, sin detalle y con escasa sensación de colores. A pesar de ello esta percepción global es extremadamente importante ya que permite al individuo tener un conocimiento general de la escena que lo rodea. ${ }^{4}$

Lo anterior es válido para la llamada visión diurna o fotópica, que ocurre para niveles "altos" de iluminación, ya que los conos son relativamente poco sensibles al estímulo luminoso. Tales niveles son los propios de la casi totalidad de las actividades humanas: diurnas en espacios abiertos (que se caracteriza por la percepción de detalles y colores) y en interiores con luz natural o iluminación artificial.

Para niveles "bajos" de iluminación (por ejemplo, una habitación en penumbra), la luz que llega a la fóvea resulta insuficiente para activar a los bastones, por lo que se pierde la imagen allí formada. La situación es conocida como visión nocturna o escotópica ${ }^{5}$ y es la que se tendría, por ejemplo, al contemplar un paisaje nocturno a la luz de la luna. No hay percepción de detalles ni sensación de colores, la imagen es tan solo de siluetas en una escala de grises, que corresponde a los estímulos nerviosos generados por los bastones. Entre ambas regiones (fotópica y escotópica) hay una zona de transición, para la cual el nivel de detalle y color se incrementa en la medida en que aumenta la iluminación.

\section{2.- Sensibilidad espectral. Color}

La longitud de onda, o rango de longitudes de onda de un rayo luminoso determina la propiedad a la que se denomina color. La capacidad de ver colores es exclusiva de la visión diurna y se debe a que los conos generan estímulos nerviosos que el sistema psíquico es capaz de interpretar como distintos, de acuerdo la frecuencia de la luz. Evidentemente, esta propiedad es común para la mayoría de las personas, de modo que, por ejemplo, casi todos asocian el rojo con frecuencias cercanas al límite superior del espectro $(760 \mathrm{~nm})$. Obviamente, la excepción a este enunciado es el conocido daltonismo;

\footnotetext{
${ }^{3}$ Bardier, D., De la Visión al Conocimiento, op. cit.

${ }^{4}$ Bardier, D., De la Visión al Conocimiento, op. cit.; De Boer, J., Fischer, D., Interior Lighting, Philips Technical Library, Deventer, 1978.

${ }^{5}$ Moon, P., The Scientific Basis... op. cit.; Holladay, L., "The fundamentals of glare and visibility", Journal of the Optical Society of America, 12(4), 1926.
} 
quienes lo sufren poseen una sensibilidad distinta y una consecuente perturbación en la visión del color. $^{6}$

La luz considerada como blanca está compuesta por radiaciones de múltiples longitudes de onda dentro del espectro visible. Cuando esta ilumina un objeto de color (por ejemplo, verde), éste reflejará las longitudes de onda correspondientes a dicho color, absorbiendo todas las restantes. De este modo se verá verde, puesto que esta luz será la que llegará al ojo.

La cualidad de diferenciar distintas longitudes de onda es propia de los conos y no de los bastones. En función de la intensidad de luz, los bastones generarán estímulos solo interpretables como distintos niveles de grises, de allí que la llamada visión nocturna sea en blanco y negro.

La intensidad de los estímulos generados por conos y bastones depende también de la longitud de onda (o frecuencia) de la luz. Cabe aquí una nueva mención a lo dicho en el punto anterior: la percepción es puramente subjetiva, por lo que no puede cuantificarse. Sin embargo, presentando a sujetos luces con determinada distribución espectral (color) y variando su intensidad hasta que el observador considerara que es igual a la de otro color tomado como referencia, puede "cuantificarse" la sensibilidad a la luz coloreada del sujeto. Experimentos como el descrito fueron realizados sobre un número grande de personas, en diversos centros de estudio, con lo que pudo concluirse que tanto para visión nocturna como para diurna existió un pico de sensibilidad, aproximadamente en el centro del espectro visible. La sensibilidad espectral decaía, luego, hacia ambos extremos: los rojos y azules. Se descubrió además una diferencia entre la sensibilidad de conos y bastones: estos últimos se mostraron ligeramente más sensibles a los azules y violetas y prácticamente insensibles a los rojos. ${ }^{7}$

El conocimiento de la sensibilidad espectral es de máxima importancia para los objetivos de esta Tesis, ya que define lo que consideramos como luz, esto es, la región visible del espectro. No todos los sistemas visuales individuales son iguales, por lo que existe un alto grado de dispersión en sus respuestas espectrales, hecho que puede considerarse como lógico si se piensa en el carácter subjetivo de la percepción. De esta manera, y con el objetivo de uniformar la definición de "luz", la Comisión Internacional de Alumbrado (CIE) estableció en 1924 una estandarización de la visión diurna (fotópica) y nocturna (escotópica) a partir de definir las curvas de sensibilidad espectral normalizadas del ojo, comúnmente conocidas como curva $\mathrm{V} \lambda$ (visión diurna) y $\mathrm{V} \lambda$ ' (nocturna). ${ }^{8} \mathrm{La}$ última revisión de estas estandarizaciones data de $2004 .^{9}$

\footnotetext{
${ }^{6}$ Conti, F., "Fisiología de la Visión”, Fisiología Médica (capítulo 18), Mc Graw Hill, 2010.

${ }^{7}$ Conti, F., "Fisiología de la Visión", op. cit.; Tomsak, R., Levine, M., Handbook of neuro-ophtalmology, ButterworthHeinemann, Oxford, 2003; Keitz, H. A. E, Light calculations and measurements, N. V. Philips, Holanda, 1974.

${ }^{8}$ Commission Internationale de l'Eclairage (CIE), [en línea] URL: http://www.cie.co.at [Consulta: 2 de abril de 2019].

${ }^{9}$ ISO 23539, 2005 (E)/CIE S 010, Photometry - The CIE system of physical photometry, 2004.
} 


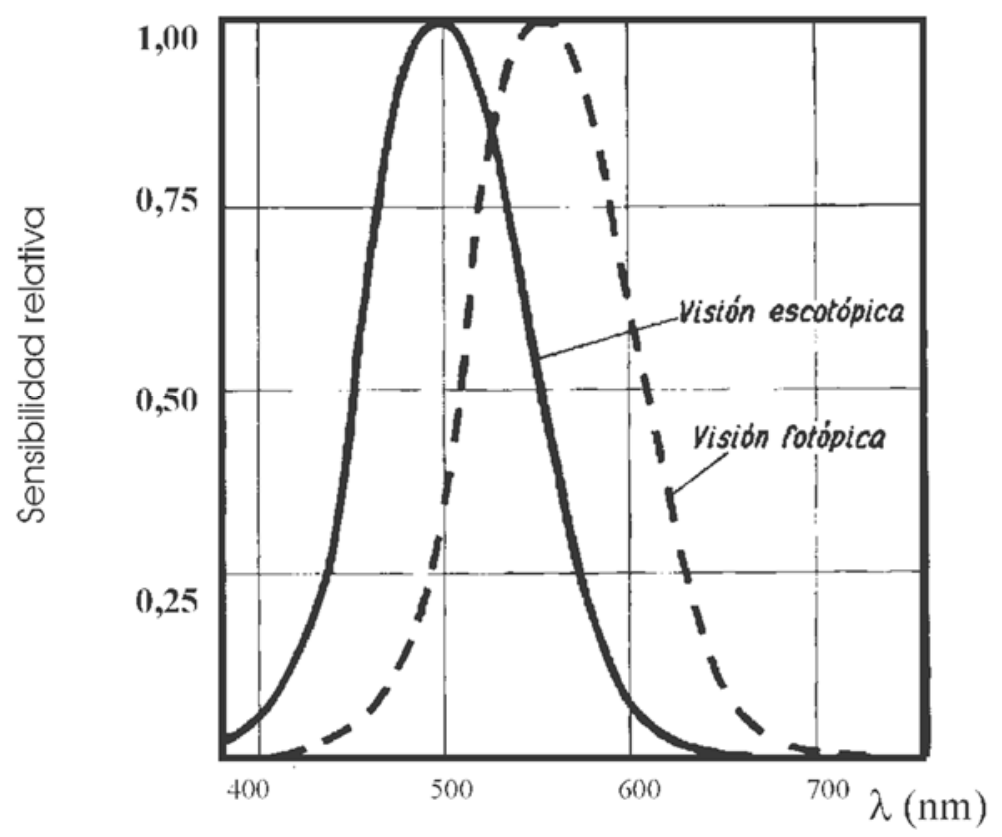

Figura 6. Sensibilidad estandarizada de conos y bastones. ${ }^{10}$

Como puede observarse en la figura 6, la sensibilidad máxima para conos se encuentra en $555 \mathrm{~nm}$ (amarillo - verdoso), con límites de visión en $380 \mathrm{~nm}$ (violeta) y aproximadamente $760 \mathrm{~nm}$ (rojos).

La curva de sensibilidad para bastones adopta la misma forma, pero se encuentra desplazada $55 \mathrm{~nm}$ hacia la zona de los azules violáceos. La figura 7 muestra en detalle la sensibilidad para la visión diurna. La curva $\mathrm{V} \lambda$ determina la cuantificación del estímulo luminoso y la definición de lo que se considera luz. Puede interpretarse de la siguiente manera: se supone una lámpara monocromática, que emite una determinada energía a $555 \mathrm{~nm}$; si dentro del mismo campo visual se coloca otra fuente, también monocromática, radiando por ejemplo a $470 \mathrm{~nm}$ (azul) y con la misma potencia, el observador la notará como 10 veces menos intensa. Dicho en otras palabras, la luz azul debería tener 10 veces más potencia que la de $555 \mathrm{~nm}$ para que sea vista con la misma intensidad.

\footnotetext{
${ }^{10}$ Imagen tomada de: Keitz, H. A. E, Light calculations... op. cit.
} 


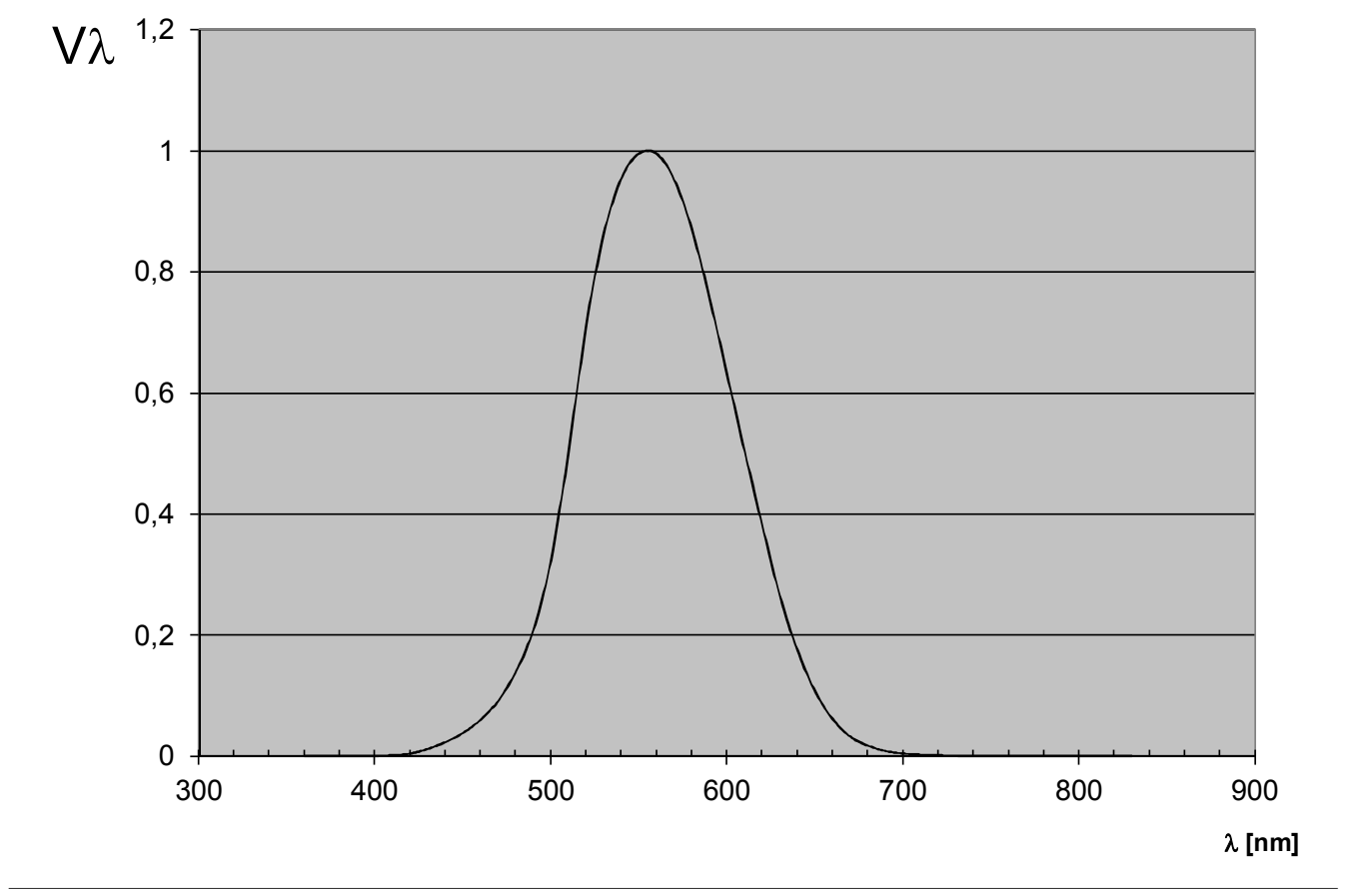

Figura 7. Curva $V \lambda .^{11}$

\footnotetext{
${ }^{11}$ Imagen basada en: ISO 23539, 2005 (E)/CIE S 010, op. cit.
} 


\section{2.- Magnitudes fotométricas}

La luminotecnia contempla una particular caracterización del estímulo luminoso y de la percepción, de modo que puedan ser modeladas, cuantificadas y predichas sus interrelaciones. Estos conceptos se definen en las llamadas magnitudes fotométricas.

Si se postula que uno de los puntos centrales de este trabajo es la caracterización de los sistemas de alumbrado vial, resulta necesario definir las distintas alternativas que se presentan al momento de cuantificar el estímulo luminoso y sus efectos, (magnitudes fotométricas), junto con sus relaciones fundamentales y su vínculo con el observador y los parámetros elegidos para calificar la calidad de una instalación de alumbrado.

Se introducen entonces, un grupo de magnitudes concebidas para cuantificar los diversos aspectos involucrados en el proceso de visión. Se presentan, por un lado, dos estrechamente vinculadas con el estímulo luminoso: Flujo e Intensidad. Por otro, un par que, de alguna manera, ponderarán la sensación visual: Iluminancia y Luminancia. Como se dijo, en tanto fenómeno subjetivo, la sensación es imposible de cuantificar o medir, sin embargo, iluminancia y luminancia se aproximarán (en unas situaciones más, en otras menos) a la percepción y serán normalmente empleadas cuando se necesiten establecer o definir niveles o límites de visibilidad.

\section{2.- Flujo luminoso}

El flujo luminoso es la cantidad de luz que emite una fuente. La unidad de medición es el lumen ( $\operatorname{lm}$ ) $\mathrm{y}$, dado los valores usuales en lámparas y luminarias, es común en la técnica el uso del múltiplo klm $\left(10^{3} \mathrm{~lm}\right)$. El flujo es una magnitud escalar, ya que solo indica la cantidad de luz generada por un dispositivo (caso típico, una lámpara), reflejada o transmitida por un cuerpo (un espejo, un vidrio refractor). No brinda información espacial, es decir, hacia dónde se dirige o de dónde proviene tal cantidad de luz. Una lámpara incandescente suspendida sobre un escritorio puede tener el mismo flujo luminoso que un artefacto de iluminación (un velador, por ejemplo). A pesar de ello, y aunque estén ubicados en la misma posición, los niveles de iluminación generados, las sombras, brillos, etc., que determinan las condiciones de visión, pueden ser completamente distintos en ambas situaciones.

Para precisar un poco más qué se entiende por cantidad de luz, puede imaginarse una fuente ideal, concentrada en un punto, que irradia una cierta potencia G. Además, esta potencia tiene una distribución espectral como la indicada, a modo de ejemplo, en la figura 8.
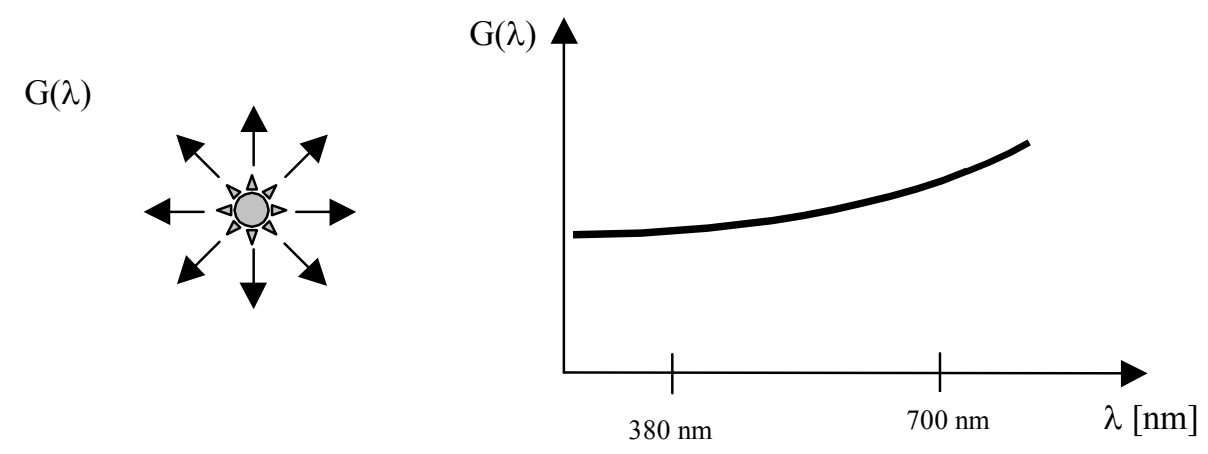

Figura 8. Fuente que irradia una potencia $G(\lambda)$ (dibujo del autor). 
Una fuente como la indicada en el diagrama emitirá en la región visible del espectro (longitudes de onda comprendidas entre aproximadamente 380 y $700 \mathrm{~nm}$ ), pudiendo también irradiar longitudes mayores a $700 \mathrm{~nm}$ (infrarrojo, ondas de radio, etc.) y menores a $380 \mathrm{~nm}$ (ultravioleta, rayos x, etc.). Si efectuáramos la sumatoria de estas radiaciones cubriendo la totalidad del espectro, obtendríamos la potencia emitida, medida en Watt (W):

$$
\mathbf{G}=\int_{0}^{\infty} G(\lambda) d \lambda \quad[W]
$$

Esta potencia no puede considerarse como "cantidad de luz", ya que incluye radiaciones no visibles. Para cuantificar el flujo luminoso debería entonces restringirse la integral solo al espectro visible. Más aún, se sabe que las radiaciones dentro de esta región $(\sim 380$ a $700 \mathrm{~nm})$ producen percepción visual, pero el ojo humano no tiene la misma sensibilidad para todas las longitudes de onda allí comprendidas. Esta sensibilidad, para niveles "altos" de iluminación (visión diurna o fotópica) ha sido estandarizada con la curva V $\lambda$, diagrama con forma de campana de Gauss, con un máximo para $555 \mathrm{~nm}$ (color verde - amarillo), y decreciente tanto para los rojos como para la zona azul del espectro.

De modo que, para obtener de todo el espectro radiado lo que se considera luz, se debería, no solo restringir la sumatoria, sino ponderar cada frecuencia con su correspondiente sensibilidad V $\lambda$.

Con esta idea, se define el flujo luminoso $\mathbf{F}$ de la fuente como la potencia radiante evaluada con respecto a la sensibilidad estándar:

$$
\mathbf{F}=\int_{0}^{\infty} v \lambda G(\lambda) d \lambda \quad[\text { vatios }- \text { luz }]
$$

La unidad resultante para el flujo es el Watt (W); para evidenciar que se trata de potencia luminosa, se emplea la denominación vatios - luz. Sin embargo y como ya se mencionó, la unidad usual de flujo luminoso es el lumen, de origen anterior a la definición de flujo dada. Considerando para la curva V $\lambda$ la estandarización de la Comisión Internacional de Alumbrado CIE (1929), la constante que surge para adaptar unidades es $683 \mathrm{~lm} / \mathrm{W}$, con lo cual:

$$
\phi=683 \int_{0}^{\infty} \operatorname{Vi} G(\lambda) d \lambda \quad[\operatorname{lm}]
$$


A riesgo de reiterar, se remarca que el flujo no es una medida de la sensación o percepción visual. Por el contrario, tal como se desprende de su definición, es una forma de cuantificar el estímulo luminoso.

A modo de ejemplo, se calcula el flujo de un led rojo, irradiando una potencia de $1 \mathrm{~W}$, cuyo espectro (simplificado) se muestra junto con la curva $V \lambda$ en el figura 9. El led, al que se considera monocromático, emite en $660 \mathrm{~nm}$, longitud de onda para la cual la sensibilidad obtenida de la curva $\mathrm{V} \lambda$ es aproximadamente 0,06 . El flujo luminoso será entonces:

$$
\phi=6830,061 \mathrm{~W}=41 \mathrm{~lm}
$$

El "rendimiento" (la denominación correcta de este concepto es eficacia) lumínico del led será de 41 $\mathrm{lm} / \mathrm{W}$. Resulta fácil deducir que la máxima eficacia teórica la tendría una fuente, también monocromática, emitiendo en $555 \mathrm{~nm}$, con $683 \mathrm{~lm} / \mathrm{W}$. Esa luz sería de color amarillo verdoso y, aunque económica desde el punto de vista del consumo energético, resultaría no muy confortable para iluminación. Como dato comparativo, una lámpara incandescente posee una eficacia de alrededor de $12 \mathrm{~lm} / \mathrm{W}$.

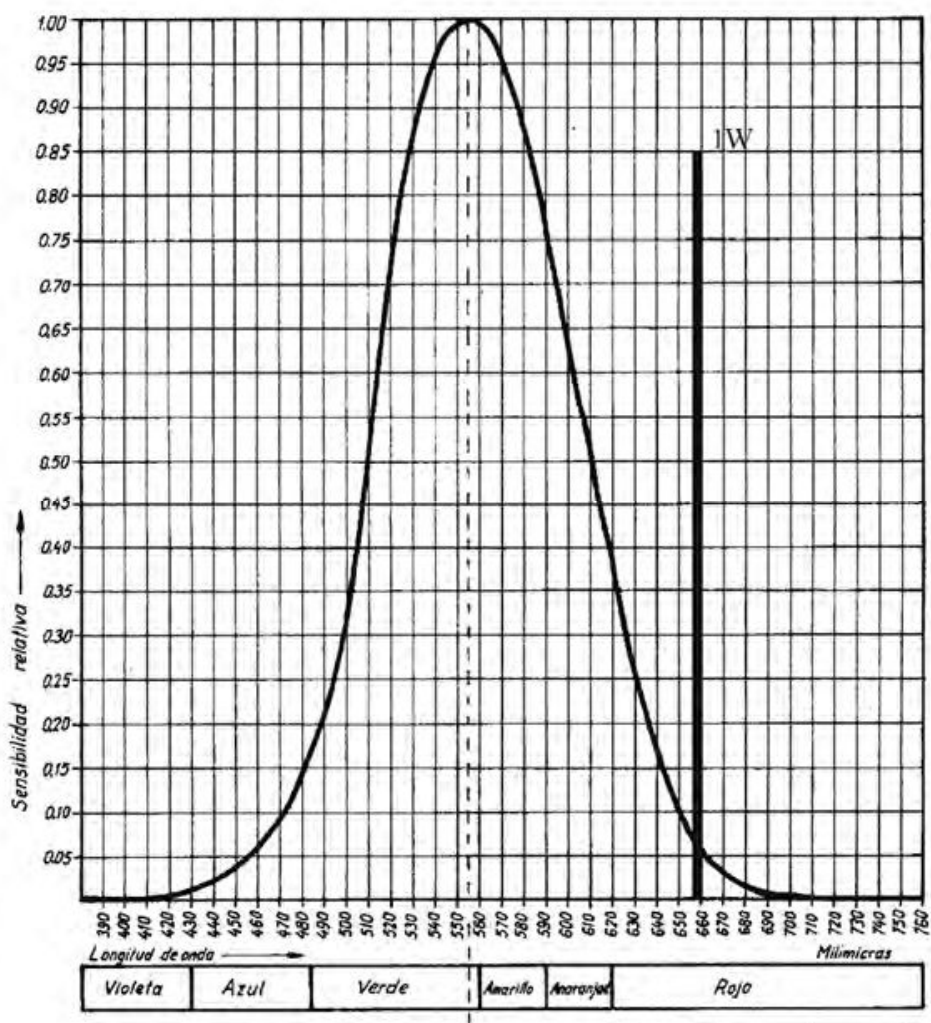

Figura 9. Emisión aproximada de un led rojo, junto con la curva V $\lambda$ (dibujo del autor).

En relación con los propósitos de investigación planteados, es conveniente considerar al flujo, medido en lumen, como una cantidad puramente física, a pesar de su dependencia de una función adoptada como estándar; este sentido será el marco para lo que sigue. No debe perderse de vista que tal definición tendrá validez solo para "altos" niveles de iluminación y para la denominada visión fotópica. 


\section{3.- Intensidad luminosa}

Si bien el flujo aporta información sobre la potencia luminosa emitida por la fuente, no dice nada sobre hacia dónde se dirige esa luz. Por esta razón, para evaluar la trayectoria de los rayos luminosos se emplea la intensidad luminosa, definida como el flujo por unidad de ángulo sólido:

$$
\mathrm{I}=\frac{\mathrm{d} \phi}{\mathrm{d} \omega} \quad[\mathrm{cd}]
$$

La unidad de medida es la candela (cd), lumen/estereorradián. La intensidad tiene sentido si además de su valor indicamos su dirección, a partir de un punto de referencia espacial situado en la fuente de luz. Por ello es normal que las intensidades de, por ejemplo, una luminaria, se informen en diagramas, del tipo mostrado en la figura 10.
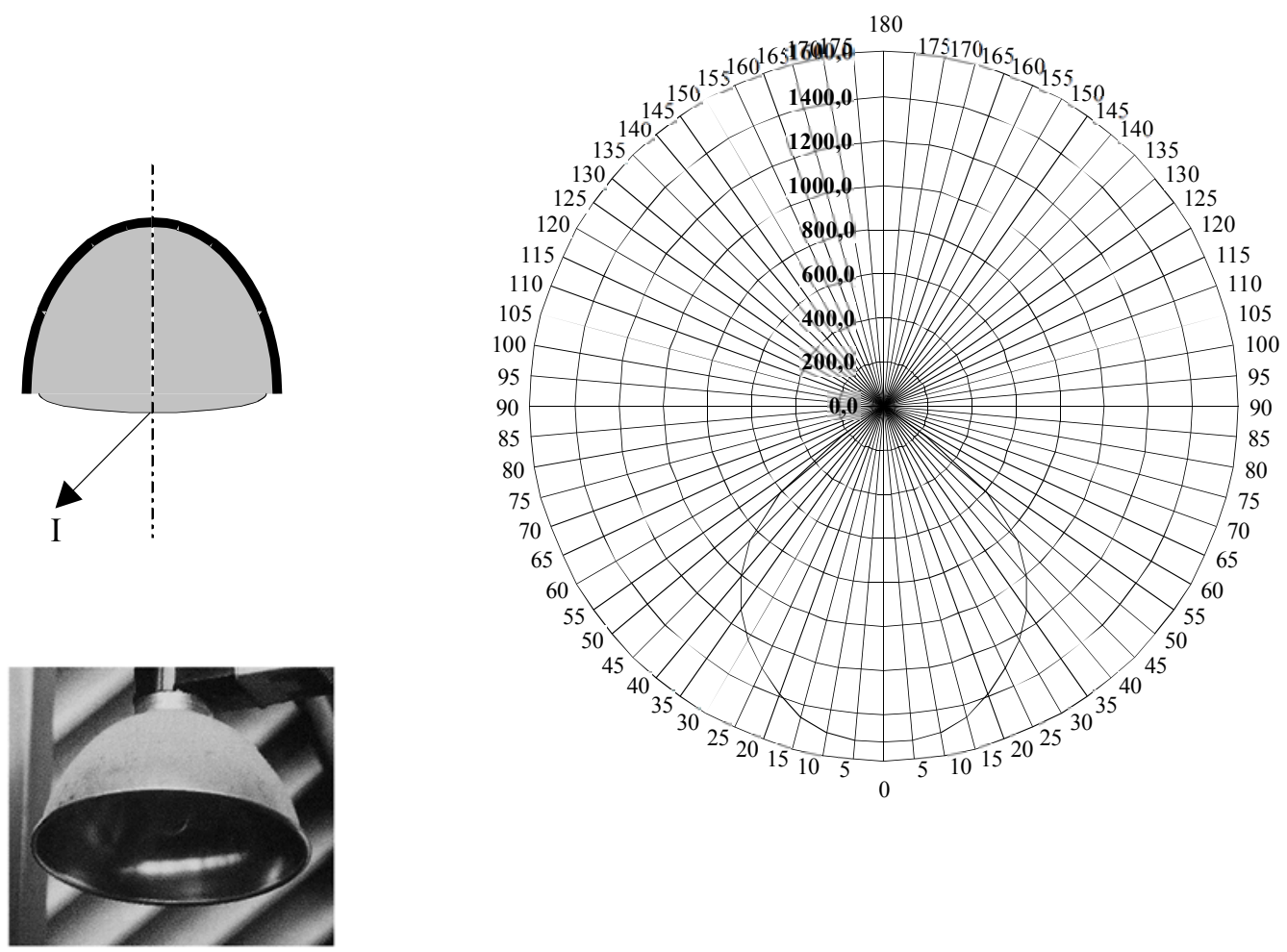

Figura 10. Diagrama polar de intensidades e imagen de la luminaria (dibujos del autor).

La curva se corresponde con una luminaria tipo "pantalla industrial", esquematizada también en la figura. Las intensidades emitidas por el artefacto pueden considerarse simétricas con respecto a un eje vertical (de rotación). Por tal motivo, solo es suficiente una curva para representarlas. En un caso más general, sería necesario contar con una familia de diagramas para describir completamente las emisiones lumínicas.

La forma de interpretar la figura 10 es la siguiente: por ejemplo, la intensidad emitida a $45^{\circ}$ con respecto a la vertical será: 


$$
\mathrm{I}=850 \mathrm{~cd}
$$

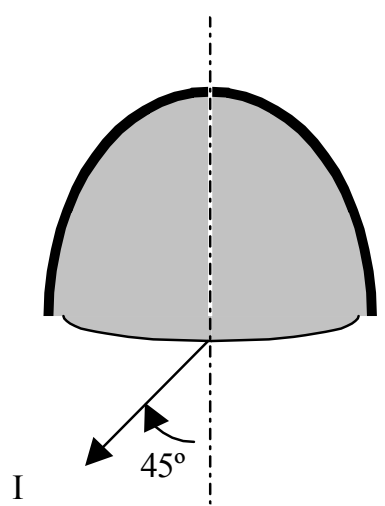

Figura 11. Interpretación del diagrama polar de intensidades (dibujo del autor).

Debe tenerse en cuenta que si bien la dirección de la intensidad es un dato necesario para su completa definición, no se trata de una magnitud estrictamente vectorial, ya que no verifica las leyes generales de, por ejemplo, adición de vectores. Sirve para interpretar una forma de indicar el rayo luminoso, considerado desde el punto de vista de la óptica geométrica, para poder realizar posteriormente cálculos o estimaciones de niveles de iluminación.

\section{3.- Relación flujo - intensidad}

Conocido el diagrama total de intensidades de una fuente luminosa, queda determinado en forma unívoca su flujo, el que puede calcularse a partir de:

$$
\phi=\int \mathrm{I} d \omega
$$

La inversa no es válida: hay infinitas combinaciones de intensidad luminosa para un mismo flujo emitido o, dicho de otra manera, el conocimiento del flujo luminoso nada dice sobre su distribución de intensidades.

Para un caso simple, una fuente que emite uniformemente, o sea, con una intensidad constante para cualquier dirección, el flujo vale:

$$
\phi=\int \text { Io } \mathrm{d} \omega \quad \phi=\operatorname{Io} \int \mathrm{d} \omega \quad \phi=\text { Io } 4 \pi
$$

De la misma manera, si la fuente emite de modo uniforme solo en un hemisferio (por ejemplo, hacia arriba), el flujo valdrá:

$$
\phi=2 \pi \mathrm{Io}
$$


Ahora bien, en la casi totalidad de los casos la intensidad no es constante, aun cuando es usual encontrar algún tipo de simetría. De todas formas, es siempre posible expresar la intensidad como función de un par de ángulos (por ejemplo, uno contenido en planos verticales como el de la figura 10, y otro para identificar cada plano). El cálculo del flujo puede expresarse entonces como:

$$
\phi=\int_{0}^{2 \pi} \int_{0}^{\pi} I(\gamma, c) d \gamma d c
$$

En la práctica, dado que normalmente I no se conoce como una función continua, sino que sus valores se obtienen (por medición) para ciertos ángulos discretos, la expresión anterior se transforma en sumatoria, existiendo métodos estandarizados para la obtención del flujo luminoso, de aplicación especialmente para el análisis de luminarias.

Finalmente, se remarca que tanto flujo luminoso como intensidad son conceptos destinados a describir y cuantificar el estímulo luminoso. De esta manera, su conocimiento no permite de por sí inferir condiciones de visibilidad. Si se tuviera el dato de la intensidad emitida por una señal luminosa (v.g., un semáforo), esa información no sería suficiente para asegurar si será o no visible, ya que la percepción no depende solo de aquel estímulo. Existen otros aspectos tales como la ubicación respecto al observador, el color, la intensidad del fondo sobre el que se ve, etc., que en conjunto con su propia intensidad posibilitarán predecir de algún modo, el grado de visibilidad.

Lo expresado en el párrafo anterior ha motivado la definición de dos magnitudes más estrechamente vinculadas con la percepción: iluminancia y luminancia.

\section{4.-Iluminancia}

Es la cantidad de luz por unidad de superficie que recibe un cuerpo. Para la superficie elemental de la figura 12, definimos la iluminancia E como:

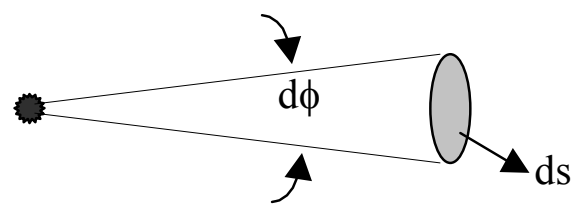

$$
\mathrm{E}=\frac{\mathrm{d} \phi}{\mathrm{ds}} \quad[\mathrm{lux}]
$$

Figura 12. Definición de iluminancia (dibujo del autor).

La iluminancia se calcula, se mide o se especifica sobre una determinada superficie o plano tomado como referencia. Así, es usual hablar de la iluminancia sobre el piso, sobre una pared, en un plano 
horizontal a determinada altura con respecto al piso. Tal plano es, obviamente, el que recibe el flujo luminoso. ${ }^{12}$

El rango usual de valores de iluminancia en la vida cotidiana es bastante amplio. Por ejemplo, sobre el piso en una noche con luna (y sin iluminación artificial), los niveles rondarán algunos décimos de lux (lx). Con algunas unidades de lux se podría caminar o trotar esquivando obstáculos, e incluso ciertas personas podrán efectuar algún deporte con poca exigencia visual, tal como jugar fútbol. Un gran porcentaje de individuos podrá leer (quizá con cierta dificultad) con poco más de 50 lx y sin inconvenientes, con más de 400 o 500 lx. En exteriores, sobre el piso, en un día soleados en horas cercanas al mediodía, la iluminación podrá superar los 50.000 lx.

A pesar de estar definida en términos de estímulo luminoso (flujo), es claro el vínculo entre iluminancia y percepción. Si pudiéramos experimentar con un gran número de individuos, un muy alto porcentaje admitiría poder leer con comodidad el diario, por ejemplo, con un nivel de $500 \mathrm{~lx}$. Asimismo, serían muy pocos los conformes si la iluminancia fuera de 50 lx y casi nadie podría ejecutar esta tarea visual con menos de 1 lx. Ahora bien, la relación es de tipo estadístico, no directa o lineal. Considerando que la iluminancia depende de la cantidad de luz que llega al cuerpo a iluminar y no la que éste refleja hacia el observador, puede concluirse que no es el mejor indicador para aproximarnos a la cuantificación de la percepción visual. Un claro ejemplo se da en la figura 13, en la que ambas letras no son igualmente visibles a pesar de iluminarse con el mismo nivel (la iluminancia actual sobre la hoja).

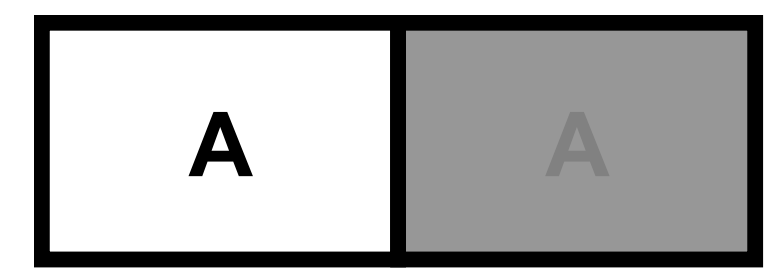

Figura 13. La igualdad en iluminancias no asegura la misma percepción. ${ }^{13}$

El sentido común indica que la percepción se relacionaría más estrechamente con una magnitud que, en lugar de cuantificar la luz que incide sobre el objeto, evaluara la luz que éste refleja hacia el observador. Tal magnitud existe y se hablará de esta en los siguientes apartados. Se adelanta aquí que esta luz reflejada es mucho más difícil de calcular o medir, porque depende de múltiples factores: tipo de superficie, rugosidad, material, color, etc., además de los ángulos de incidencia y reflexión de la luz. Es por estos motivos (simplicidad en el cálculo y medición) que en la mayoría de los casos se prefiere la iluminancia al momento de tener que especificar o definir niveles de iluminación para las diversas actividades visuales.

\section{5.- Relación Intensidad luminosa - Iluminancia. Ley de la inversa de los cuadrados}

\footnotetext{
${ }^{12}$ De Boer, J., Fischer, D., Interior Lighting, op. cit.

${ }^{13}$ Basado en: Bardier, D., De la Visión al Conocimiento, op. cit.
} 
Se supone una fuente puntual que ilumina un área elemental ds (figura 14). Se entiende por puntual a aquella fuente cuya mayor dimensión es mucho más pequeña que la distancia que la separa de la superficie que ilumina.

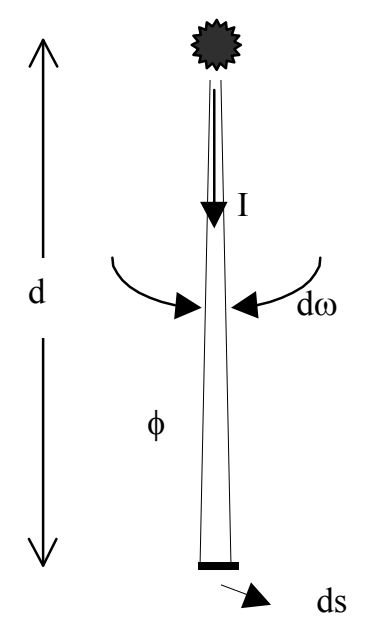

Figura 14. Ley de la inversa de los cuadrados (dibujo del autor).

En estas condiciones, la iluminancia en dicha área valdrá:

$$
E=\frac{d \phi}{d s}
$$

Y el flujo comprendido en el ángulo sólido que abarca a la superficie será:

$$
\mathrm{d} \phi=\mathrm{I} \mathrm{d} \omega
$$

Para el esquema de la figura, el ángulo sólido es el cociente entre la superficie del casquete esférico cubierto $(\approx \mathrm{ds})$ y el radio al cuadrado:

$$
d \omega=\frac{d s}{d^{2}}
$$

Con lo cual:

$$
E=\frac{I}{d^{2}}
$$

Esta ecuación expresa que la iluminancia sobre un plano perpendicular a la línea de incidencia de la luz decrece con el cuadrado de la distancia que lo separa de la fuente luminosa. 
La ley de inversa del cuadrado es de gran importancia en la luminotecnia, tanto en lo referente a cálculos como en la especificación de sistemas y métodos de medición. Más aun, es conceptualmente útil re-definir la intensidad luminosa como capacidad de una fuente de generar niveles de iluminación, como modo de independizarla de la noción, no siempre simple de visualizar, de ángulo sólido. Matemáticamente, esta nueva concepción de intensidad se expresa como: ${ }^{14}$

$$
I=\lim _{d \rightarrow \infty} E d^{2}
$$

\section{6.- Ley del coseno}

Se considera la situación planteada en la figura 11, en donde la luz incide en forma oblicua al plano que ilumina.

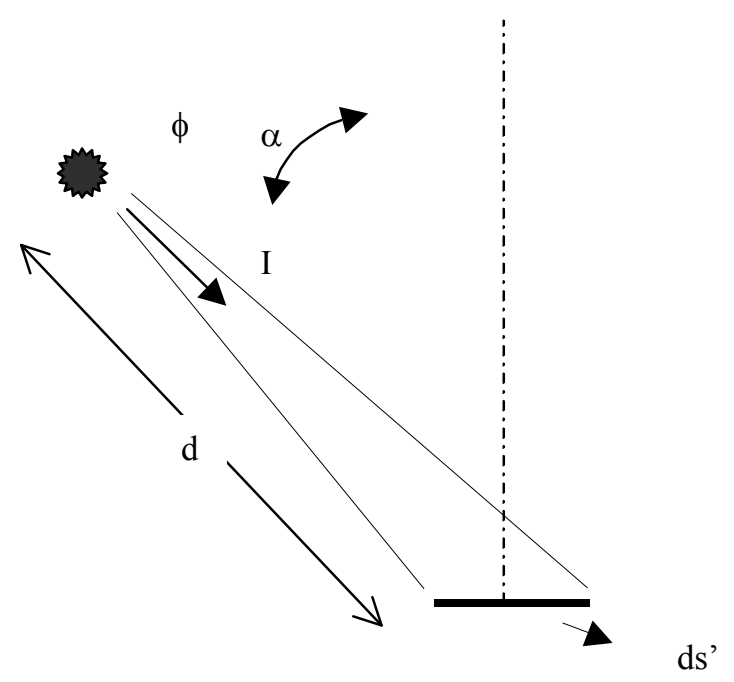

Figura 15. Rayo luminoso que incide en forma oblicua (figura del autor).

El flujo luminoso contenido en el mismo ángulo sólido de la situación anterior (figura 15) se distribuye ahora sobre una superficie mayor ds'. Su relación con ds es:

$$
\mathrm{ds}^{\prime}=\frac{\mathrm{ds}}{\cos \alpha}
$$

Por lo que la iluminancia valdrá:

$$
E=\frac{\phi}{d s}=\frac{I}{d^{2}} \cos \alpha
$$

\footnotetext{
${ }^{14}$ Moon, P., The Scientific Basis..., op. cit.
} 
Esta es la llamada ley del coseno. ${ }^{15}$ Comúnmente las luminarias (fuentes luminosas) se ubican a una altura constante con respecto al nivel del piso. Si llamamos $\mathrm{H}$ a tal distancia medida en forma vertical, la ecuación anterior puede transformarse en:

$$
E=\frac{I}{H^{2}} \cos ^{3} \alpha
$$

Esta es la expresión más práctica de la ley para las aplicaciones usuales.

\section{7.- Luminancia}

Cuando dos fuentes de la misma intensidad luminosa, una de las cuales tiene un área mayor que la otra, se miran sucesivamente, la menor se percibe como más "brillante". Esta noción de brillo aparece muy ligada a la percepción: recortados sobre un mismo fondo, un objeto "brillante" será más fácil de detectar que otro calificado como "opaco". En tanto aproximación a esta idea de brillo, se ha definido la luminancia L como la intensidad luminosa emitida por unidad de superficie:

$$
L=\frac{\mathrm{dI}}{\mathrm{ds}}[\mathrm{cd} / \mathrm{m} 2-\text { nit }]
$$

En la ecuación anterior, "dI" representa la intensidad luminosa emitida por el elemento de superficie "ds", por lo que L remite a la luminancia en un punto (infinitesimal). Al igual que el caso de la intensidad luminosa, la luminancia, si bien es una magnitud escalar, requiere de una posición (de observación) de referencia. La figura 16 aclara este punto: para una observación normal a la superficie, la luminancia media es el cociente entre la intensidad emitida en promedio (Io) y la superficie que la emite.

\footnotetext{
${ }^{15}$ Moon, P., The Scientific Basis..., op. cit.; Keitz, H. A. E, Light calculations..., op. cit.
} 


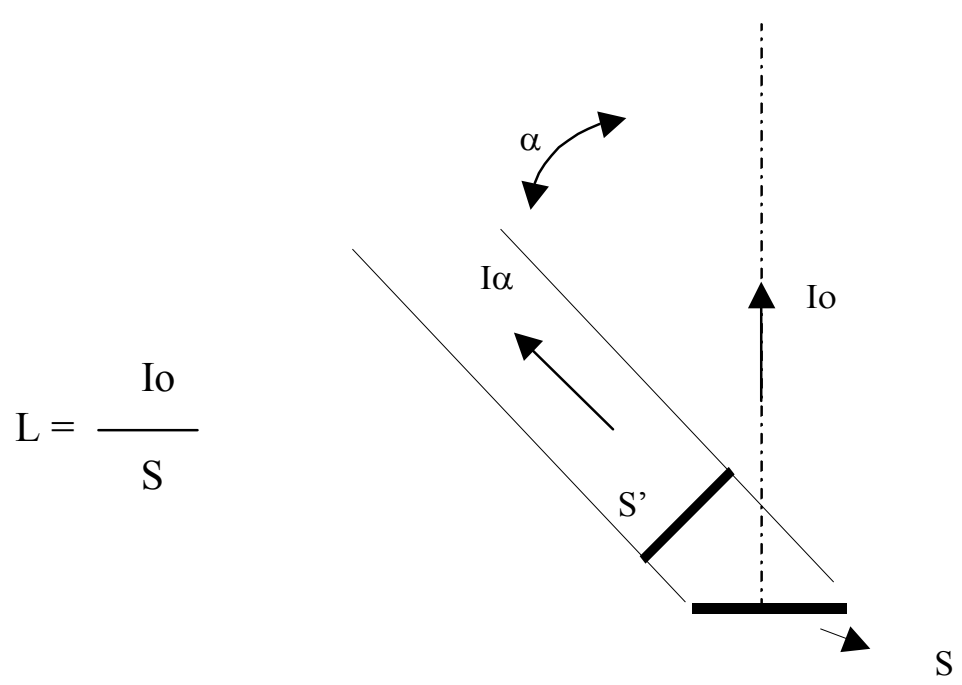

Figura 16. Definición de luminancia (dibujo del autor).

La superficie a considerar es siempre la que ve el observador o superficie aparente de la fuente. En la figura 16, para una dirección de observación oblicua en un ángulo $\alpha$ con respecto a la vertical, la luminancia sería:

$$
\mathrm{L}=\frac{\mathrm{I} \alpha}{\mathrm{S}^{\prime}}
$$

\section{8.- Ley de Lambert}

Cuando un cuerpo emite o refleja luz de modo tal que su luminancia es constante independientemente del ángulo de observación, se dice que emite (o refleja) en forma uniforme o según la ley de Lambert. ${ }^{16}$

Retomando el esquema de la figura 16, si la emisión fuera uniforme se estaría ante:

$$
\mathrm{L}=\frac{\mathrm{I} \alpha}{\mathrm{S},}=\frac{\mathrm{Io}}{\mathrm{S}}
$$

debiéndose entonces verificar que,

$$
\mathrm{I} \alpha=\text { Io } \cos \alpha
$$

${ }^{16}$ Keitz, H. A. E, Light calculations..., op. cit.; Illuminating Enineering Society (IES), The Lighting Handbook, 9na. edición, McGraw-Hill, Estados Unidos, 2011. 
El sol es un ejemplo de cuerpo emisor según la ley de Lambert. Se advierte comúnmente que su imagen es la de un disco plano, con un brillo prácticamente homogéneo, pero en realidad, se está observando una semiesfera, o sea, una superficie desde variados ángulos $\alpha$ y para todos con igual luminancia.

Para ángulos de incidencia y observación no muy rasantes $\left(\alpha<60-50^{\circ}\right)$, las superficies consideradas "mates" (un cielorraso de yeso, una pared pintada satinada) se aproximan bastante a la reflexión uniforme.

\section{9.- Reflexión - Reflexión uniforme}

Si cierto flujo luminoso $\phi i$ incide sobre una superficie, parte de éste se reflejará ( $\phi r)$ al medio. Es posible expresar entonces la siguiente relación:

$$
\phi \mathrm{r}=\rho \phi \mathrm{i}
$$
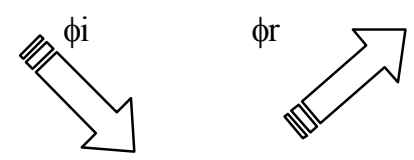

Figura 17. Reflexión de la luz.

El factor $\rho$ se denomina coeficiente de reflexión o reflectancia y obviamente es menor que uno. Para la gran mayoría de las superficies, $\rho$ no es una constante, sino que depende de los ángulos de incidencia y reflexión de la luz. Tan solo puede considerarse independiente de éstos para el caso de superficies que cumplen con la ley de Lambert, conocidas como perfectamente difusoras o reflectoras uniformes.

En el caso de superficies perfectamente difusoras, se establece la siguiente relación:

$$
\phi r=\int \mathrm{I} \alpha \mathrm{d} \omega
$$

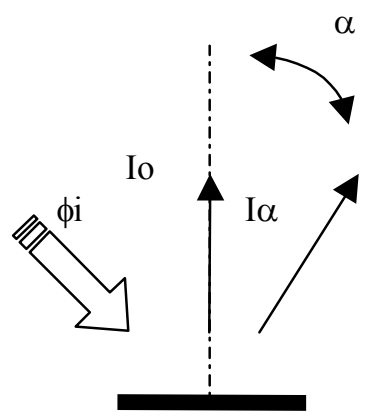

$\mathrm{S}$

Figura 18. Relación intensidad - flujo en reflectores perfectos (figura del autor).

Como la luminancia es constante, debe verificarse la relación entre Io (constante) e I $\alpha$, por lo que la integral se transforma en: 


$$
\int \operatorname{Cos} \alpha d \omega=\pi
$$

Con lo que:

$$
\phi \mathrm{r}=\mathrm{Io} \pi=\rho \phi \mathrm{i}
$$

Si se dividen ambos términos de la igualdad por la superficie $\mathrm{S}$ que recibe (y refleja) la luz se obtendrá, por un lado, flujo incidente sobre superficie (iluminancia); por otro, intensidad emitida en dirección perpendicular a S, por unidad de área (luminancia):

$$
\mathrm{L}=\frac{\rho}{\pi} \mathrm{E}
$$

La relación anterior es de gran utilidad puesto que relaciona una magnitud fácil de calcular y medir (iluminancia), con otra muy vinculada a la visión pero compleja de obtener, cuya medición involucra equipos de alto costo. No debe perderse de vista su campo de aplicación: solo superficies que puedan asimilarse a difusores o reflectores perfectos.

\section{3.- Fotometría}

Con el término fotometría se designa a la medición de las magnitudes que caracterizan la emisión de luz visible por parte de una fuente luminosa (flujo e intensidad luminosa), y a la medición de aquellas que de alguna manera describen la sensación visual (iluminancia y luminancia).

Como ya se comentó, el aspecto clave las instalaciones de alumbrado vial es la luminancia sobre la calzada, su distribución, su medición y cálculo. De modo que el estudio de las diversas alternativas de medición (la fotometría) y en especial la medición de luminancias (que se abordará en el último punto) resultan necesarios para avanzar en el objeto de la presente Tesis.

En fotometría, se acostumbra a hacer una distinción entre fotometría visual y fotometría física.$^{17}$ En la primera de ellas, prácticamente en desuso hoy en día, se empleaba la gran capacidad del ojo humano para detectar diferencias de luminancias. Utilizando métodos comparativos, se podía medir de este modo la totalidad de las magnitudes fotométricas. ${ }^{18}$

La fotometría física se basa en el uso de transductores, que transforman la luz en alguna magnitud eléctrica de fácil medición. Las fotocélulas de barrera constituyen los transductores empleados en la casi totalidad del instrumental fotométrico actual, aunque para ciertas aplicaciones especiales se utilizan los llamados tubos fotomultiplicadores. También se puede mencionar a los bolómetros, tubos de vacío en los cuales la energía radiante calienta un conductor delgado, haciendo variar su resistencia, y los termopares, que generan una fuerza electro motriz (f.e.m.) cuando su temperatura

\footnotetext{
${ }^{17}$ Moon, P., The Scientific Basis..., op. cit.; Keitz, H. A. E, Light calculations..., op. cit.

${ }^{18}$ Walsh, J., Photometry, Constable \& Company Ltd., Londres, 1958.
} 
se eleva por efectos de la radiación. Finalmente, existen dispositivos que si bien no poseen características de estabilidad tales que permitan ser empleados en instrumental de medida, sí se usan ampliamente como elementos de control o conmutación electrónica (por ejemplo los fotodiodos, los fototransistores y las fotorresistencias).

\section{1- Fotocélulas de barrera}

Las fotocélulas de barrera constan de una unión metal-semiconductor, construida de modo tal que la luz puede incidir sobre la juntura formada (figura 19). Ante la incidencia de luz, se generan portadores libres que, si el circuito exterior lo permite, producen una corriente eléctrica. Inicialmente (década del '30), las fotoceldas se construían empleando selenio como semiconductor, aunque paulatinamente fueron reemplazadas por silicio, un material de mejores características.

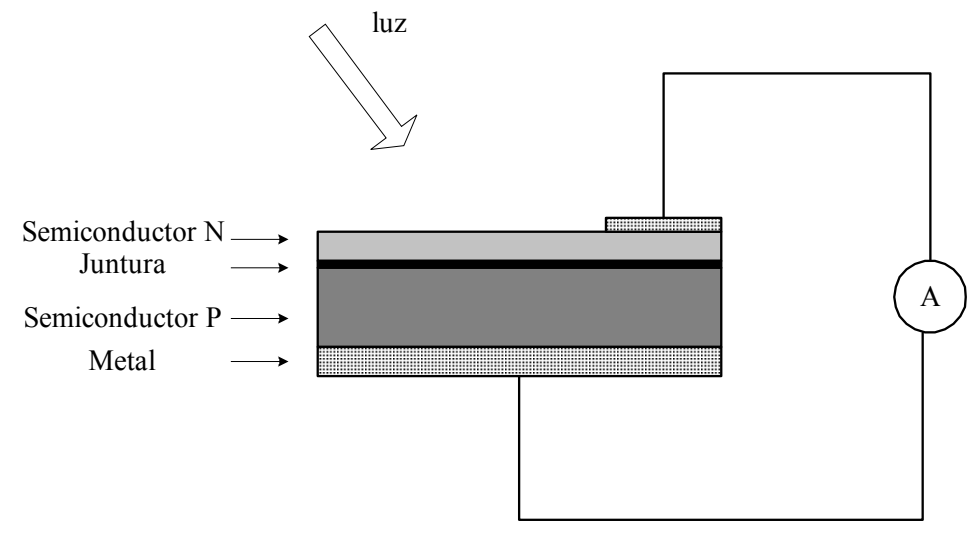

Figura 19. Esquema de la unión (dibujo del autor).

La corriente generada por este transductor resulta proporcional al flujo luminoso que incide sobre la celda por unidad de superficie, en otras palabras, la corriente de salida es proporcional a la iluminancia sobre el elemento. El instrumento básico resulta entonces un medidor de iluminancias o luxímetro, cuyo esquema elemental se muestra en la figura 20:

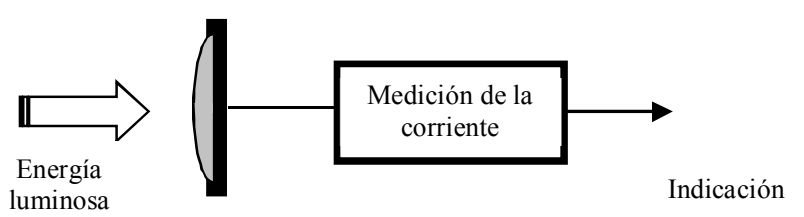

Figura 20. Esquema elemental de un luxímetro (figura del autor). 
La medición del resto de las magnitudes fotométricas (intensidad y flujo luminoso, luminancia) se realiza luego de transformarlas en una cantidad proporcional a la iluminancia sobre el elemento fotosensible.

La corriente que genera la fotocelda depende del valor de la resistencia conectada en el circuito exterior. En la figura 21 se muestran algunas curvas típicas. La corriente aumenta en forma perfectamente lineal con el nivel de iluminación sólo si la fotocelda se encuentra en corto circuito. Para las exactitudes típicas de instrumentos portátiles $(3 \%-5 \%)$, suelen conseguirse linealidades aceptables si la resistencia de carga es menor que algunas centenas de ohm. En cuanto a los valores de corriente generada, depende del área de la fotocelda que ha sido expuesta a la luz. Para los tamaños usuales, la corriente puede alcanzar algunos mA para iluminaciones del orden de 500 a 1000 lx. En estos casos, la medición de la corriente puede efectuarse directamente con un miliamperímetro, que incluso provee la baja impedancia necesaria para lograr linealidad en la respuesta.

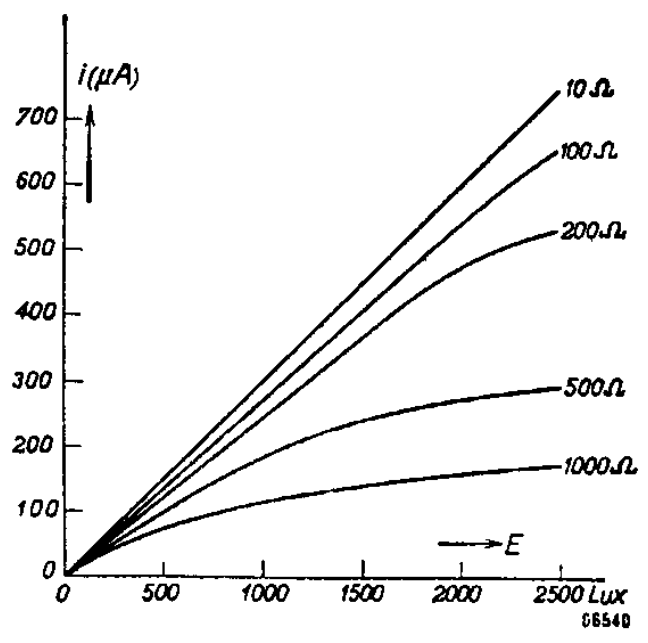

Figura 21. Respuesta de una fotocelda. ${ }^{19}$

Otro punto de interés en la fotometría es la estabilidad de la relación iluminancia - corriente de salida. En este sentido, las fotoceldas de barrera mantienen sus curvas de respuesta por largos períodos. Para las exactitudes usuales del instrumental fotométrico, suele ser suficiente realizar calibraciones anuales luego de la calibración inicial. Entre los factores que pueden alterar esta estabilidad se mencionan, la temperatura, la fatiga del elemento fotosensible y las condiciones de uso (en especial la humedad). En cuanto al primer punto, basta pensar que la energía térmica genera en la juntura portadores libres, que se sumarán a la foto corriente. Lo común es que esta "corriente térmica" se haya incluido en la calibración del instrumento, indicándose (en la hoja de datos del mismo) un determinado rango de temperatura en el que valen los errores especificados, junto con factores para la corrección en otras condiciones de uso. En instrumentos más sofisticados (de laboratorio), se consigue una mayor estabilidad mediante la llamada "termostatización" del elemento fotosensible. La fotocelda se aloja

\footnotetext{
${ }^{19}$ Imagen tomada de: Walsh, J., Photometry, op. cit.
} 
en un pequeño recinto calefaccionado, en el que la temperatura se mantiene constante y ligeramente superior a la ambiente. ${ }^{20}$

Con el término "fatiga" se hace referencia a la paulatina pérdida de sensibilidad del elemento fotosensible. Ésta es más acentuada en la medida en que la fotocelda se expone a altos niveles de iluminación, aunque los niveles propios del uso del instrumento también la provocan. Una buena práctica para minimizar este efecto es mantener cubierto al elemento cuando el instrumento no está siendo usado. ${ }^{21}$

Finalmente, condiciones tales como golpes, suciedad, etc., pueden alterar la calibración del sistema de medida. En especial la humedad ambiente, durante el uso del aparato o cuando éste se encuentra guardado, puede llegar a dañar al elemento fotosensible o al filtro con él provisto (las características y función del mismo se mencionarán en los puntos siguientes).

\section{2- Fotomultiplicadores}

Los tubos fotomultiplicadores son válvulas de vacío cuyo cátodo emite electrones ante la incidencia de luz. Poseen una serie de grillas auxiliares que, gracias al fenómeno conocido como emisión secundaria, incrementan la cantidad de electrones emitidos inicialmente, de modo de obtener en el ánodo una corriente que resulte fácil de medir.

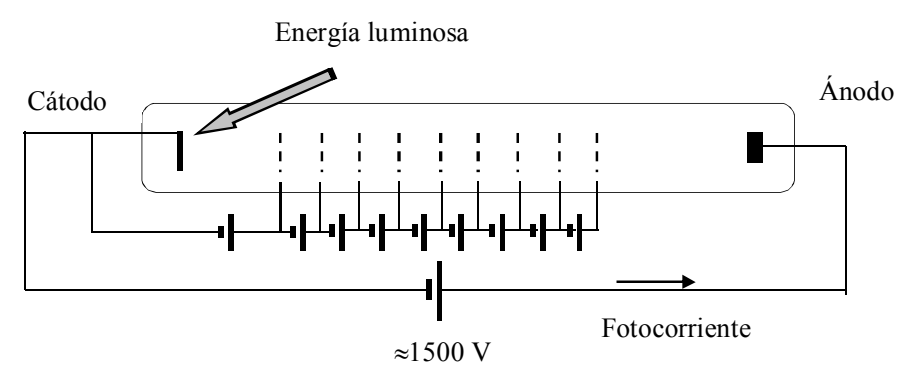

Figura 22. Esquema del fotomultiplicador (dibujo del autor).

La corriente generada resulta, al igual que las fotoceldas de barrera, directamente proporcional al nivel de iluminancia sobre el cátodo, pudiéndose conseguir una mayor linealidad. La principal desventaja de este tipo de transductores es que necesitan una fuente de alta tensión (del orden de los $1500 \mathrm{~V}$ ) para su alimentación, que sea muy estable, puesto que afecta directamente a la fotocorriente. Además, tienen muy poca estabilidad, por lo que el instrumento debe poseer algún sistema que

\footnotetext{
20 "Photometric Sensor Catalog", Gamma Scientific, [en línea] URL: http://www.gamma-sci.com/products/photometricsensors/ [Consulta: 2 de abril de 2019].

"Photometer Heads", LMT Lichtmesstechnik GMB, [en línea] URL: http://www.lmt-berlin.de/en/pheads.html [Consulta: 2 de abril de 2019]. http://www.everfine.net/en/products.php?fid=1\&cid=8 [Consulta: 10 de abril de 2019]

${ }^{21}$ Keitz, H. A. E, Light calculations..., op. cit.; De Boer, J., Van Bommel, W., Road Lighting, Philips technical Library, Países Bajos, 1980.
} 
permita calibrarlo antes de cada medición. Ambos factores contribuyen a incrementar notablemente el costo de los sistemas de medición que emplean fotomultiplicadores. Como contrapartida, se logra con ellos una muy alta sensibilidad, quedando de esta forma su uso reservado a equipos que deban medir niveles de iluminación muy pequeños (casi exclusivamente luminancímetros).

\section{3- Medición de iluminancia}

El medidor de iluminancias (luxímetro o luxómetro) es el equipo básico en fotometría ya que como se verá, en él se basa la medición del resto de los parámetros fotométricos (intensidad, flujo y luminancia).

El luxímetro es el instrumento fotométrico más simple, puesto que se aplica sólo para medir adecuadamente la corriente generada por el transductor. Si se piensa en un instrumento analógico, el esquema planteado en la figura 23 puede mejorarse de la siguiente manera:

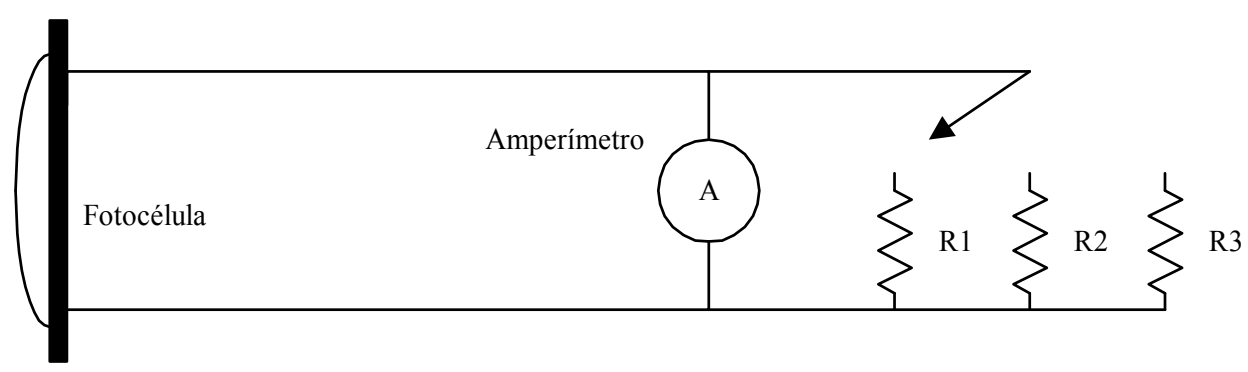

Figura 23. Luxímetro analógico (dibujo del autor).

El miliamperímetro proporciona la condición de baja impedancia a la salida del transductor, necesaria para lograr una buena linealidad. En la medida que la luz incidente aumenta, será necesario derivar parte de la corriente generada por la fotocelda, para no sobrepasar el rango de medida del miliamperímetro. Esto se logra con el uso de los derivadores marcados como R1, R2, etc., que contribuyen por otra parte a disminuir la impedancia conectada al elemento fotosensible.

En un luxímetro digital debe conectarse a la salida de la fotocelda un circuito que convierta corriente en tensión. Esto es así ya que los conversores analógico digitales (que constituyen la primera etapa de los instrumentos digitales) miden esta última magnitud. El conversor corriente - tensión provee además la baja impedancia a la salida de la celda. 


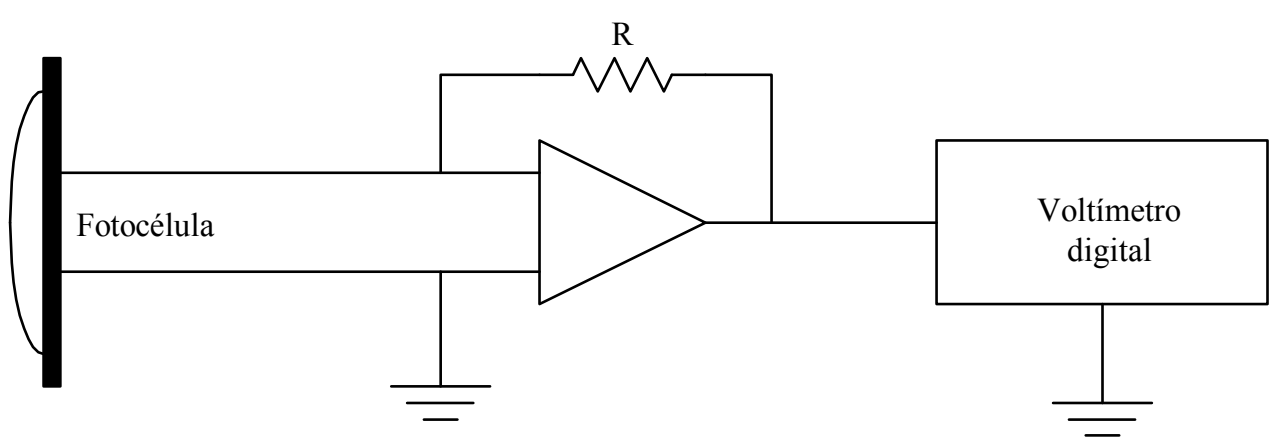

Figura 24. Luxímetro digital (dibujo del autor).

Los esquemas de conexión mencionados se emplean en la casi totalidad de los luxímetros portátiles, diseñados para medir adecuadamente niveles de iluminancia por encima de 50 o $100 \mathrm{~lx}$. Para la medición de niveles menores (en instrumentos con escalas de 1 lx o menos), suele ser necesario agregar una etapa que amplifique el corriente, previo a su medición. La justificación se encuentra en la baja sensibilidad de los transductores de silicio o selenio y en el hecho de que no es conveniente que el elemento fotosensible sea de gran tamaño.

La elección entre un instrumento analógico o uno digital pasa exclusivamente por las ventajas que estos últimos implican en cuanto a la facilidad en la lectura y posterior tratamiento de los valores medidos (por ejemplo guardarlos en una memoria digital o transferirlos a una computadora).

En cuanto a la exactitud, los errores de las fotoceldas empleadas en los instrumentos portátiles rondan el \pm 5 o $\pm 10 \%$. Si se tiene en cuenta que, con instrumentos de bajo costo (tanto analógicos como digitales) una corriente puede medirse con errores menores al $\pm 1 \%$, se concluye que la elección entre un luxímetro digital o analógico no pasa por la exactitud.

\section{4- Calibración}

Para calibrar o tarar al instrumento es preciso generar sobre el elemento fotosensible niveles conocidos de iluminancia, adaptando en forma conveniente la escala de corriente del amperímetro de modo que los valores se registren directamente en lux.

Para tal fin se utiliza un equipo denominado banco fotométrico, que consta de un soporte largo en forma de riel, con posibilidad de instalar en uno de sus extremos una lámpara patrón de intensidad luminosa. Sobre el riel se monta la fotocelda, de modo tal que la intensidad conocida de la lámpara patrón incida sobre ella en forma normal. El equipo se completa con un sistema que permite medir la distancia entre la lámpara y el elemento fotosensible (figura 25). 


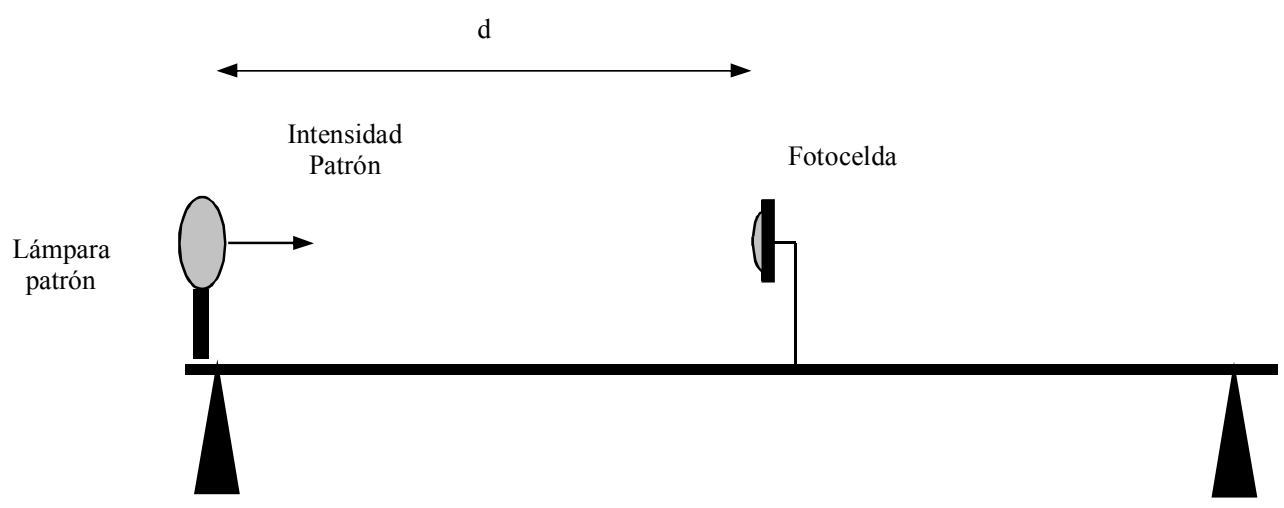

Figura 25. Banco fotométrico (dibujo del autor).

En estas condiciones, el nivel de iluminancia que podemos considerar como patrón, se obtiene a partir de:

$$
\mathrm{E}=\frac{\mathrm{I}}{\mathrm{d}^{2}}
$$

La distancia d puede variarse (desplazando sobre el riel al montaje del elemento fotosensible), con lo que se logran varios niveles de iluminancia para una misma intensidad patrón. Hay que remarcar que la ecuación anterior (denominada ley de la inversa de los cuadrados) vale en la medida que la separación entre el elemento a calibrar y la fuente luminosa sea suficientemente mayor al tamaño de los mismos, lo que generalmente se logra con distancias d mayores a 1,5 o $2 \mathrm{~m}$.

En cuanto a la lámpara patrón, las normas indican que debe ser incandescente, con una temperatura de color de $2860 \mathrm{~K}^{22}$

\section{5- Adaptación a la respuesta espectral normalizada $V(\lambda)$}

Luego de su calibración, es de esperar que el instrumento mida correctamente iluminancias, independientemente de las características (distribución espectral) de la fuente luminosa que las genera. Para lograr este propósito es necesario que la respuesta espectral del elemento fotosensible sea la curva normalizada $\mathrm{V}(\lambda)$. Tanto las fotoceldas de silicio o selenio, como los fotomultiplicadores, tienen sensibilidades espectrales que van más allá del espectro visible, por lo que es posible adaptar su respuesta mediante el uso de filtros coloreados. Hay distintas tecnologías para la construcción de estos filtros, pudiendo ser de vidrio, estar conformados por la superposición de láminas delgadas de material plástico, e incluso ser de líquidos de alta densidad o gel. Además, dado que la sensibilidad espectral de cada elemento fotosensible es una característica particular, el filtro de adaptación debe ajustarse en forma individual, no siendo intercambiables.

\footnotetext{
${ }^{22}$ ISO/CIE 19476, 2014 (E), Characterization of the Performance of Illuminance Meters and Luminance Meters, 2014.
} 
El filtro de adaptación a la curva $\mathrm{V}(\lambda)$ es una de las partes más delicadas de cualquier instrumento fotométrico, incluyendo los luxímetros. La transmitancia del mismo puede modificarse ante cambios bruscos de temperatura, exposición a niveles muy altos de iluminación o la humedad ambiente, alterando no solo la respuesta espectral del instrumento, sino también su calibración. Se puede incluso decir que la falta de adaptación (por un deterioro o mala calidad del filtro) es la principal fuente de error de un luxímetro, con el agregado adicional de que es una falla difícil de detectar. En efecto, dado que el instrumento se calibra con luz incandescente, cuando se miden fuentes de este tipo es muy probable que los errores caigan dentro de lo tolerable, aunque la adaptación no sea buena. Sin embargo, para lámparas de descarga (de espectro no continuo), la exactitud puede bajar a límites inadmisibles, según sea el espectro de radiación de la misma. ${ }^{23}$

Finalmente, los grados de adaptación varían, dependiendo de la calidad y del uso previsto para el luxímetro. Los hay simplemente adaptados (figura 26), que incluso pueden requerir del uso de factores de corrección para la medición de cierto tipo de fuentes luminosas; con adaptación fina y muy fina, en donde la curva del elemento fotosensible se acerca mucho o coincide casi perfectamente con la respuesta normalizada $\mathrm{V}(\lambda)$. Lógicamente los costos se incrementan notablemente en la medida que la adaptación mejora, reservándose el último grado para instrumentos de laboratorio.

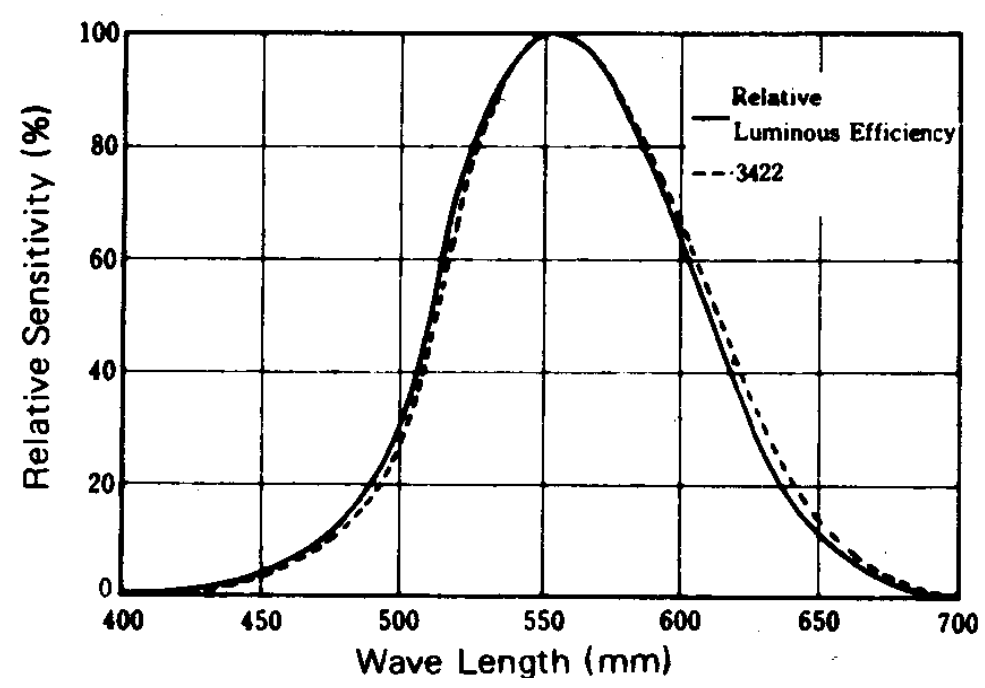

Figura 26. Adaptación a la curva $V(\lambda)$ para un luxímetro portátil (línea de trazos). ${ }^{24}$

\section{6- Adaptación a la ley coseno}

Otra de las características que debe poseer un luxímetro es medir correctamente la iluminancia independientemente de la ubicación de la fuente de luz que la genera. Como se sabe, si la luz incide sobre una superficie en forma oblicua, la iluminancia sobre la misma es proporcional al coseno del ángulo de incidencia (figura 27).

\footnotetext{
${ }^{23}$ Keitz, H. A. E, Light calculations..., op. cit.; Illuminating Enineering Society (IES), The Lighting..., op. cit.

24 "Lux meter FT3424", HIOKI, [en línea], URL: https://www.hioki.com/en/products/detail/?product key=5659 [Consulta: 2 de abril de 2019].
} 


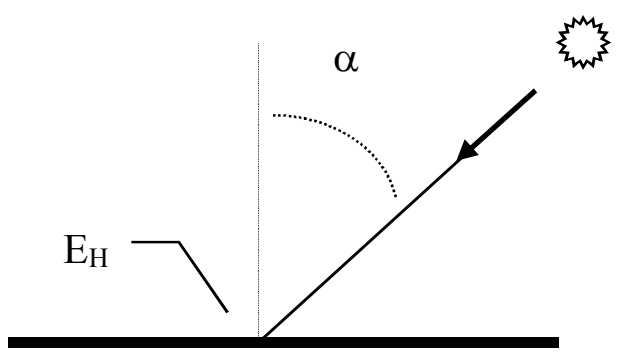

$\mathrm{E}_{\mathrm{H}}=\mathrm{E}_{\alpha} \cos \alpha$

Figura 27. Ley del coseno. E⿺: iluminancia en un plano perpendicular al rayo luminoso (Dibujo del autor).

La fotocelda por sí misma dista mucho de cumplir con esta ley, llegando incluso a reflejar la luz en caso de incidencia muy oblicua. La corrección se consigue adicionando una cubierta a modo de sistema óptico, calculada especialmente para lograr la respuesta buscada. En la mayoría de los casos es de material opalino, de forma aproximada a un casquete esférico, aunque la corrección también puede obtenerse con una o varias capas de vidrio transparente plano.

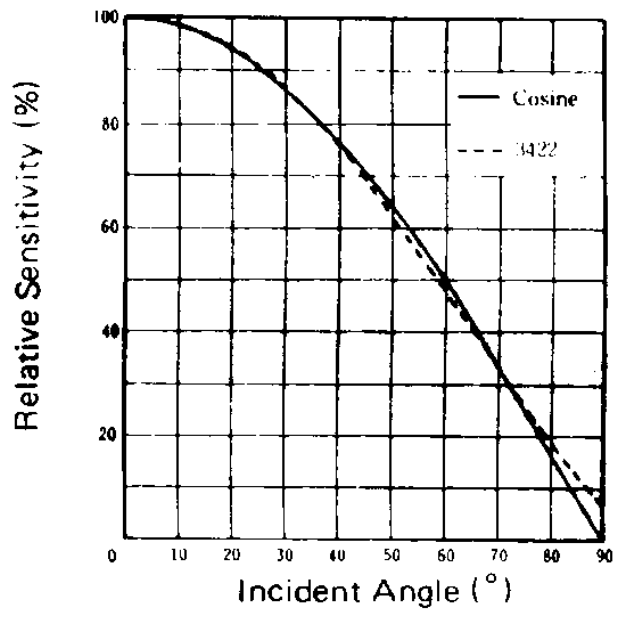

Figura 28. Adaptación a la ley coseno para un luxímetro portátil (línea de trazos). ${ }^{25}$

25 "Lux meter FT3424", op. cit. 


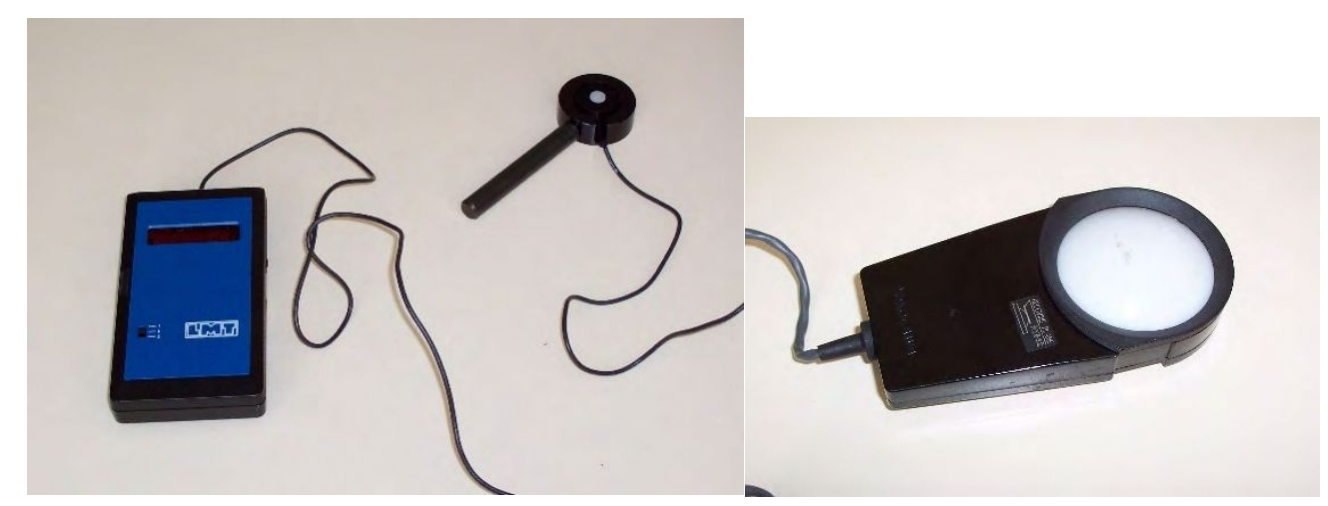

Figura 29. Tipos de cubiertas del fotodetector (fotos del autor).

Con estas cubiertas se logran buenas respuestas angulares para incidencias de hasta 60 o $70^{\circ}$; para ángulos mayores (salvo luxímetros muy sofisticados) lo normal es tener apartamientos de la ley coseno del orden del 20 o 30\%. Esta fuente de error debe ser correctamente evaluada en aquellos casos en los que la iluminancia a medir es producida exclusivamente por fuentes situadas en tales direcciones, siendo de menor importancia cuando, por ejemplo, se mide la iluminación de un interior. En este caso, la luz provendrá de varias direcciones (oblicuas, pero también cercanas a la normal), por lo que el error se minimizaría.

\section{7- Medición de iluminancias}

Cuando se efectúa un relevamiento de niveles de iluminación a partir de la medición de iluminancias, es conveniente tener en cuenta los puntos siguientes: ${ }^{26}$

- El luxímetro debe estar correctamente calibrado. Prácticamente la totalidad de los fabricantes de instrumentos indican una calibración anual, la que debe incluir el control de la respuesta espectral y la corrección de acuerdo con la ley coseno.

- El instrumento debe ubicarse de modo que registre la iluminancia que interesa medir. Ésta puede ser horizontal (v.g. para determinar el nivel de iluminancia media en un ambiente) o estar sobre una superficie inclinada (un tablero de dibujo). Debe tenerse siempre presente cuál es el plano de referencia del instrumento, el que suele marcarse directamente sobre la fotocelda o indicarse en su manual.

- Se debe tener especial cuidado en excluir de la medición aquellas fuentes de luz que no sean de la instalación. Asimismo, es necesario evitar producir sombras sobre el sensor del luxímetro.

- En el caso de instalaciones con lámparas de descarga, es importante que éstas se enciendan al menos veinte minutos antes de realizar la medición, para permitir una correcta estabilización. Suele ser importante registrar el valor de la tensión de alimentación de estas lámparas.

- En instalaciones con lámparas de descarga nuevas, éstas deben estabilizarse antes de la medición, lo que se logra luego de entre 100 y 200 horas de funcionamiento.

\footnotetext{
${ }^{26}$ De Boer, J., Fischer, D., Interior Lighting, op. cit.; Tomsak, R., Levine, M., Handbook of neuro-ophtalmology, op. cit.; Keitz, H. A. E, Light calculations..., op. cit.; Illuminating Enineering Society (IES), The Lighting..., op. cit.
} 
- Cuando se evalúa iluminancia media, debe cuidarse la elección de la cantidad y la ubicación de los puntos de medición. Por lo general se trata de definir una cuadrícula o grilla, distribuyendo los puntos en forma regular. En el caso de mediciones en interiores, suele ser suficiente con que la distancia entre puntos sea tres o cuatro veces menor que la separación entre luminarias.

\section{8- Medición de la intensidad luminosa}

La intensidad luminosa se mide siguiendo el principio de funcionamiento del banco fotométrico. La fuente cuya intensidad se quiere medir se coloca a suficiente distancia de una fotocelda, que mide la iluminancia producida sobre un plano normal al rayo luminoso. La intensidad se calcula entonces como:

$$
\mathrm{I}=\mathrm{E} * \mathrm{~d}^{2}
$$

Por lo general interesa medir las intensidades emitidas en todas las direcciones por lámparas o luminarias (su distribución de intensidades), por lo que éstas se colocan sobre un soporte que permite abatirlas espacialmente (goniómetro). Los hay de varios tipos: directos, con espejos, con distinta ubicación de los ejes, etc., pero todos trabajan sobre la misma idea. El goniómetro debe permitir el movimiento de la luminaria de modo que todos los rayos luminosos emitidos por la fuente puedan, en algún momento, incidir normalmente en la fotocelda, indicando además las coordenadas angulares de cada rayo.

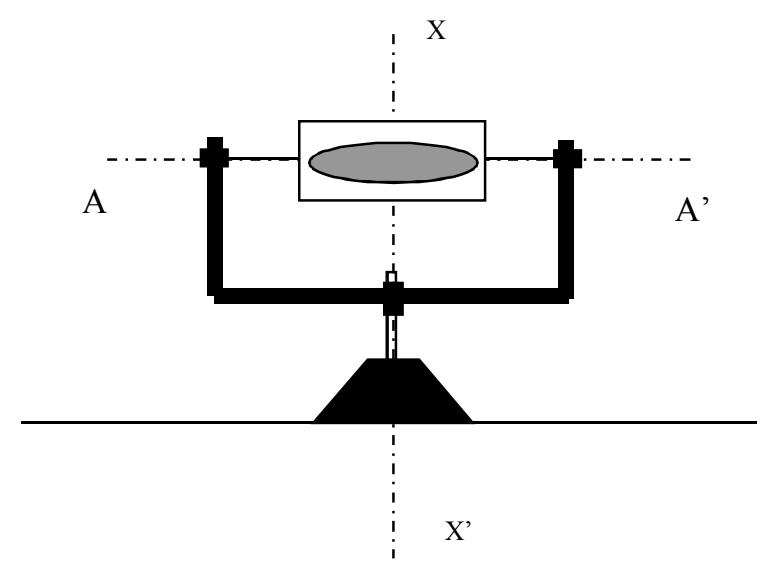

Figura 30. Esquema de un goniómetro (dibujo del autor).

En la figura 30 se ha esquematizado un ejemplo de goniómetro directo. La luminaria puede girarse sobre el eje XX' (mediante el arco soporte) y sobre el eje AA' situado sobre el arco. Para este ejemplo, la fotocelda se ubicaría sobre una tercera dimensión, saliendo del dibujo y apuntando a la intersección de los ejes XX' y AA'.

La distancia entre el goniómetro y la fotocelda (brazo fotométrico) depende del tamaño de la fuente a medir, debiendo ser suficientemente mayor, según lo que indica la ley de la inversa de los cuadrados. Lo usual es que el brazo fotométrico sea mayor a $15 \mathrm{~m}$, llegando hasta 30 o $40 \mathrm{~m}$ cuando se miden 
luminarias de gran tamaño (por ejemplo, proyectores para iluminación de estadios deportivos o luminarias dobles para alumbrado vial).

En cuanto a la fotocelda, debe poseer la adaptación a la curva $\mathrm{V}(\lambda)$ (para evaluar correctamente intensidades provenientes de fuentes con distinta distribución espectral) y dado que siempre recibirá incidencia normal, no es necesaria la adaptación a la ley del coseno. La distribución luminosa de una luminaria es un dato que interesa para posteriores cálculos (por ejemplo, de niveles de iluminación), por lo que debe obtenerse con adecuada exactitud. Esto impone determinadas características a la fotocelda: estabilidad (debe ser termostatizada), adecuada sensibilidad (para medir intensidades pequeñas) y bajo error. Los elementos fotosensibles que mejor se adaptan para esta función son los de silicio, y suelen alojarse dentro de un tubo apantallado con el objeto de evitar la medición de luz dispersa (figura 31 ). El conjunto se denomina cabezal fotométrico. ${ }^{27}$

El equipo compuesto por cabezal, brazo fotométrico y goniómetro se conoce como goniofotómetro.

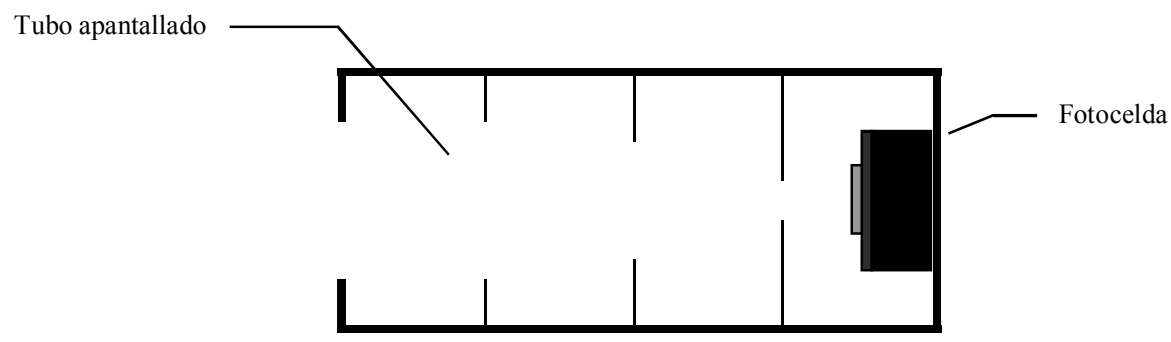

Figura 31. Cabezal fotométrico (dibujo del autor).

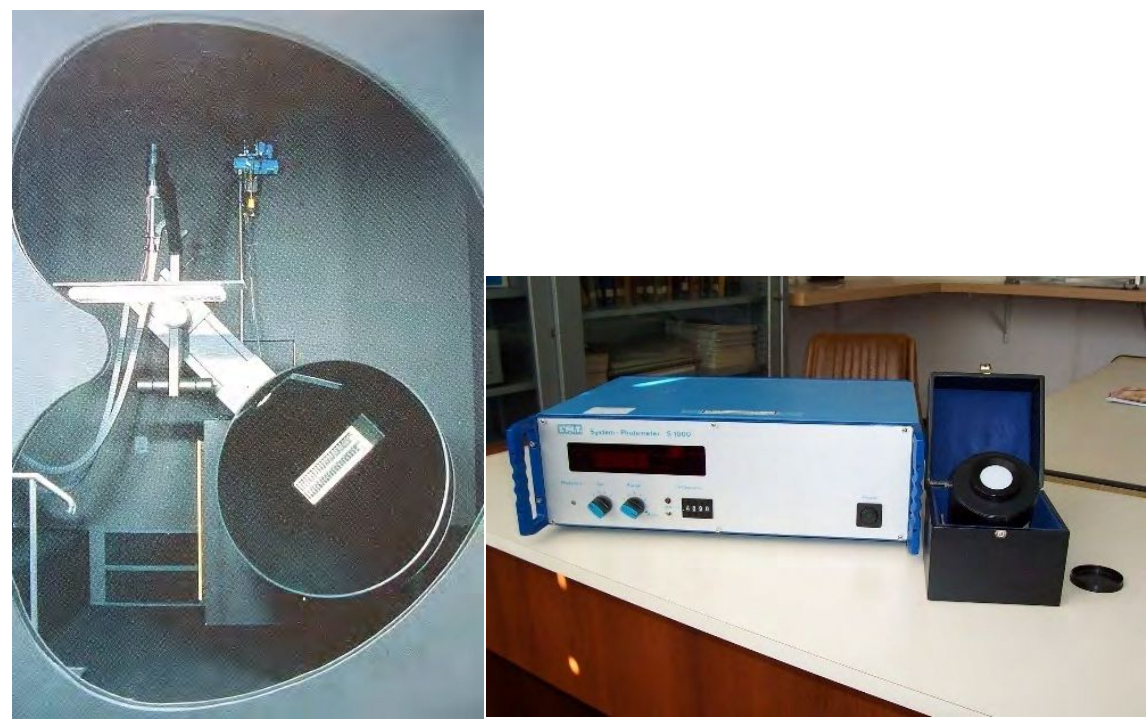

Figura 32. Goniómetro de espejo, medidos y cabezal fotométrico. ${ }^{28}$

\footnotetext{
${ }^{27}$ Keitz, H. A. E, Light calculations..., op. cit.; Walsh, J., Photometry, op. cit.

${ }^{28}$ Imágenes tomadas de: "Photometer Heads", op. cit.
} 


\section{9- Medición de flujo luminoso}

El flujo luminoso emitido por una lámpara o luminaria puede obtenerse por cálculo, partiendo de su distribución luminosa. Para ello hay que montar el equipo en un goniofotómetro, midiendo luego las intensidades luminosas. Con estos datos se efectúa la integración necesaria para obtener el flujo luminoso. A pesar que el proceso puede automatizarse, es relativamente lento y demora, como mínimo, un par de horas (incluyendo la tarea de centrado de la lámpara o luminaria en el goniómetro, que suele ser laboriosa).

Existe un método alternativo, más simple, y que se adapta especialmente a la medición del flujo luminoso de lámparas. Se basa en el uso de la llamada esfera integradora, que es una cavidad cuya particularidad es que su superficie interior puede considerarse un difusor perfecto.

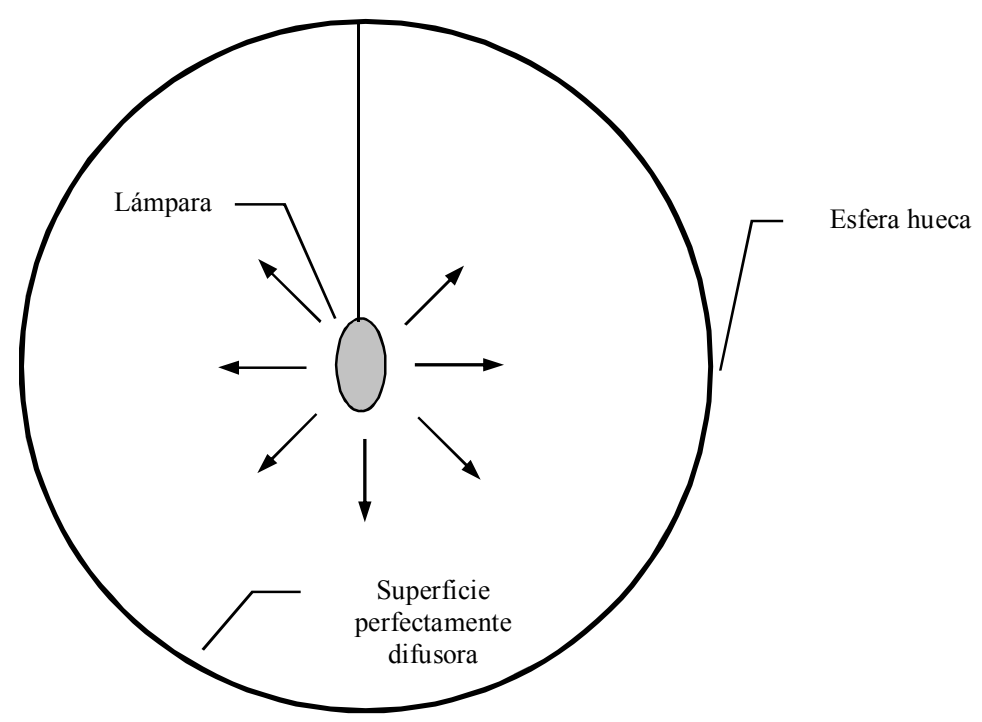

Figura 33. Esfera integradora (dibujo del autor).

La iluminancia en el interior de la esfera, producida por el flujo directo de la lámpara, puede calcularse como:

$$
E_{1}=\frac{\phi}{4 \cdot \pi \cdot R^{2}}
$$

$\mathrm{R}$ es el radio de la esfera. Luego de incidir sobre la pared, la luz se refleja, incidiendo nuevamente sobre la misma. La iluminancia producto de esta primera reflexión valdrá:

$$
E_{2}=\frac{\rho \cdot \phi}{4 \cdot \pi \cdot R^{2}}
$$

Allí, $\rho$ es la reflectancia de la superficie interior de la esfera. La luz se refleja nuevamente en la pared, y así continua indefinidamente. La iluminancia total del interior será: 


$$
E=\frac{\phi}{4 \cdot \pi \cdot R^{2}}+\frac{\rho \cdot \phi}{4 \cdot \pi \cdot R^{2}}+\frac{\rho^{2} \cdot \phi}{4 \cdot \pi \cdot R^{2}}+\ldots . .=\frac{\phi}{4 \cdot \pi \cdot R^{2}} \cdot \frac{1}{1-\rho}
$$

De la ecuación anterior se deduce que podemos conocer el flujo luminoso de la lámpara a partir de la medición de la iluminancia en la pared de la esfera. Para ello, se realiza un orificio en el que se coloca el elemento fotosensible, para el que vale todo lo dicho en el punto sobre medición de intensidad luminosa, con la salvedad que debe poseer corrección cosenoidal.

La iluminancia directa $\left(E_{l}\right)$ puede estar afectada por irregularidades propias de la fuente de luz (sombras del soporte del tubo de descarga, falta de simetría del filamento, etc.), por lo que es conveniente evitar su medición. Este es el propósito de una pantalla que se coloca entre la fuente luminosa y el orificio de medición (figura 19). Con este agregado, la relación entre iluminancia y flujo se transforma en:

$$
E=\frac{\phi}{4 \cdot \pi \cdot R^{2}} \cdot \frac{\rho}{1-\rho}
$$

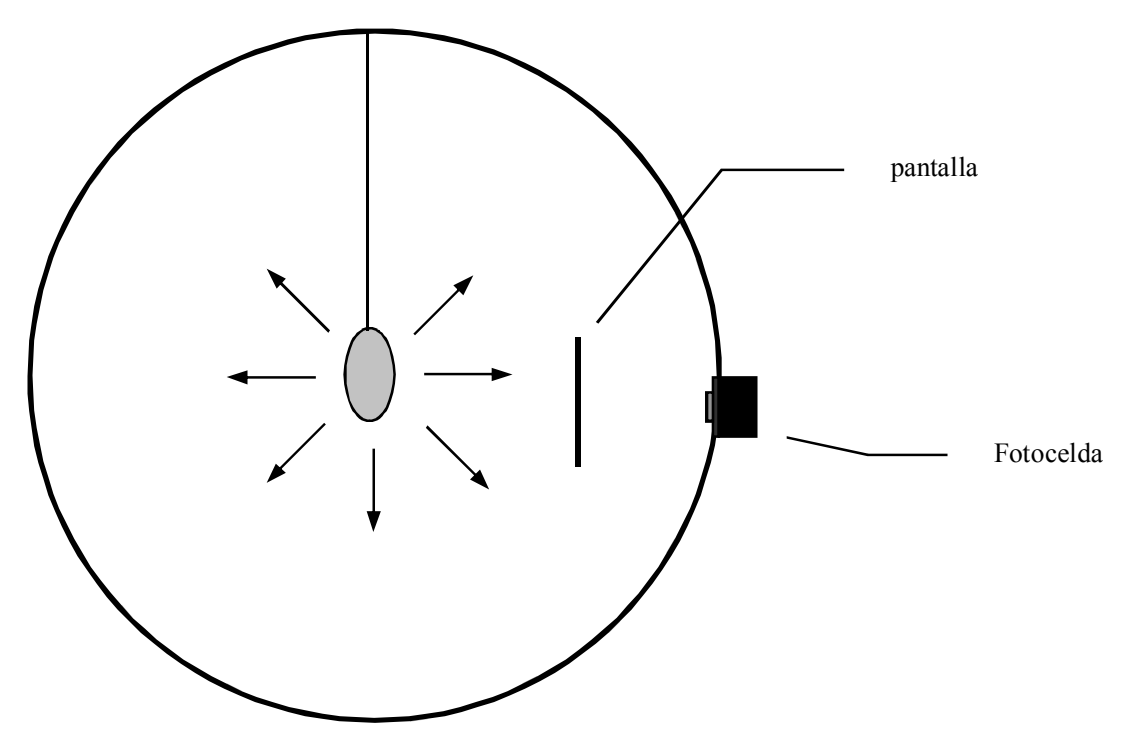

Figura 34. Esfera integradora. Ubicación de la pantalla y la fotocelda (dibujo del autor). 


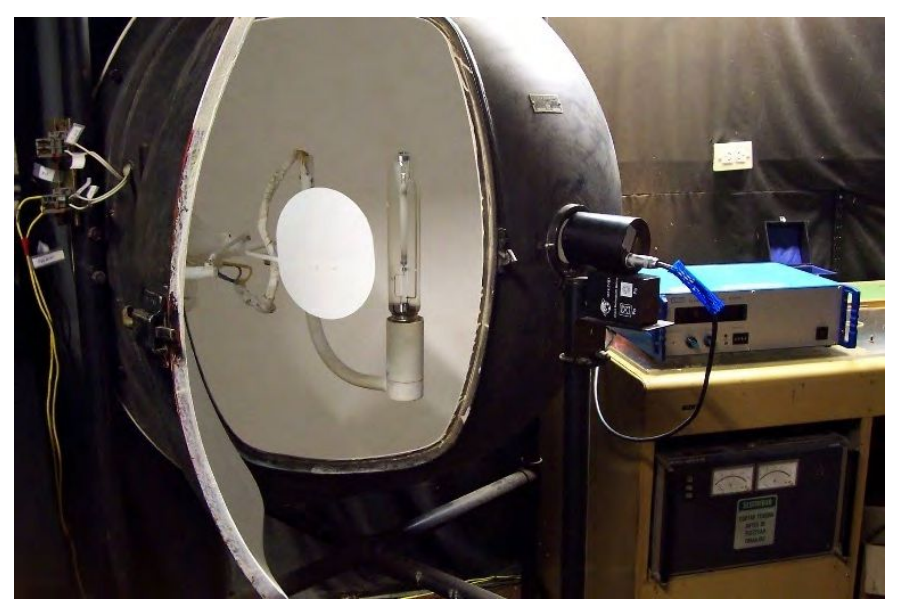

Figura 35. Esfera integradora (foto archivo LAL).

En un integrador real aparecen numerosas fuentes de error, a saber: falta de homogeneidad del recubrimiento interior, la selectividad cromática del mismo, la absorción por parte de los elementos que se encuentran en el interior (cables, soportes, etc.), etc. Todos ellos deben tenerse en cuenta y compensarse cuando se calibra el instrumento.

\subsection{0- Medición de luminancia}

Para el caso de rutas rápidas y autopistas, la normativa nacional sigue a las Recomendaciones de la Comisión Internacional de Alumbrado CIE. Éstas emplean como parámetro de calidad del alumbrado a la luminancia sobre la calzada, basada justamente en su estrecho vínculo con la percepción visual (el tema se desarrollará en extenso en el próximo capítulo). De este modo, la medición de luminancias es un punto central para el trabajo en esta Tesis y gran parte de los estudios posteriores tienen su base en los fundamentos que se discuten a continuación.

En la figura 36 se esquematiza una fotocelda, enfrentada a una superficie (S) de luminancia $L$. Entre ambas se ha intercalado un diafragma (D), con un ángulo de abertura $(\alpha)$ :

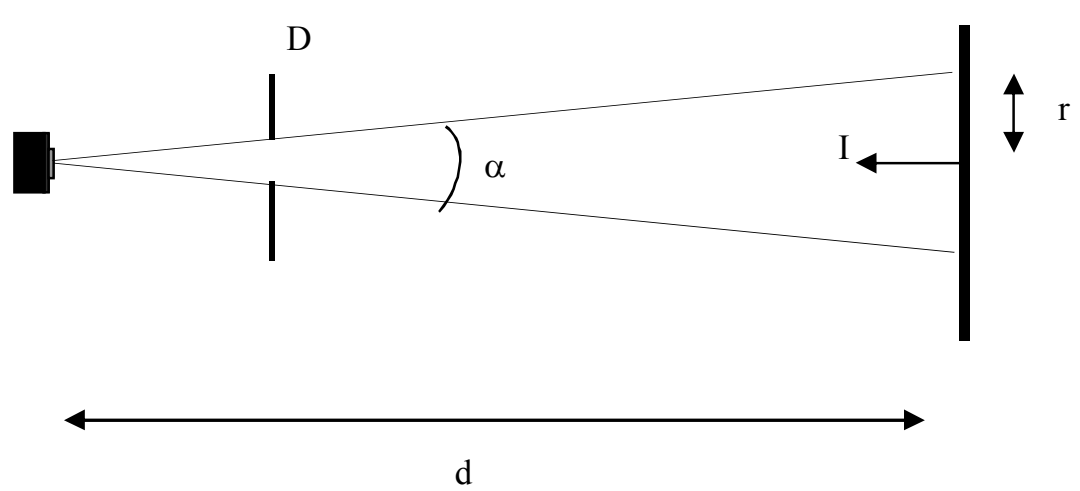

Figura 36. Principio del luminancímetro (esquema del autor). 
El diafragma limita la luz que ingresa al elemento fotosensible, de modo que éste "ve" una superficie iluminada cuya área vale $\pi r^{2}$, y cuya luminancia será:

$$
L=\frac{I}{\pi \cdot r^{2}}
$$

Por otra parte, la fotocelda medirá una iluminancia $E$ que valdrá:

$$
E=\frac{I}{d^{2}}
$$

Como:

$$
\operatorname{tg}(\alpha / 2)=\frac{r}{d}
$$

Obtenemos que:

$$
L=\frac{E}{\pi \cdot \operatorname{tg}(\alpha / 2)}
$$

En otras palabras, el hecho de limitar el campo de la fotocelda hace que su indicación sea proporcional a la luminancia de la porción de superficie "visible" a través del diafragma. En un instrumento real, se agrega un sistema óptico con el cual es posible obtener una imagen de la zona cubierta por el diafragma. El conjunto recibe el nombre de luminancímetro y medirá la luminancia promedio de dicha superficie visible.

Cuando se toman lecturas con un luminancímetro, es importante cubrir con el diafragma una superficie ligeramente menor que la de la fuente de luminancia que se pretende medir. Con este propósito, los equipos se diseñan con varios diafragmas de distintas aberturas angulares, los que pueden intercambiarse fácilmente. Las aberturas típicas van desde 2 o 3 grados, hasta el orden de los minutos.

Si se piensa en un luminancímetro usado con el diafragma pequeño (por ejemplo, 2'), se comprenderá por qué es necesario que la fotocelda posea elevada sensibilidad. Si bien existen modelos que emplean fotoceldas de silicio, los luminancímetros más poderosos son los que utilizan tubos fotomultiplicadores. 


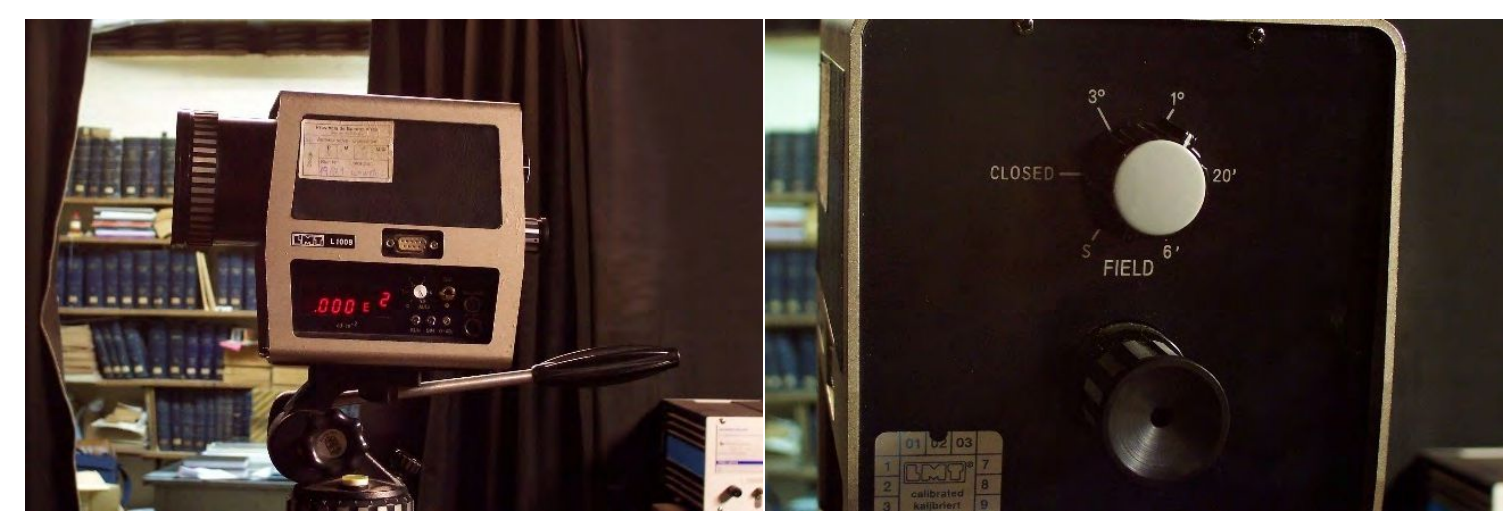

Figura 37. Luminancímetro. Detalle del ajuste de aperturas (Fotos del autor). 
Capítulo 2 - Alumbrado Público y Vial 
Luego de plantear en el capítulo precedente los fundamentos de la luminotecnia, que abarcan nociones de fisiología de la visión, magnitudes fotométricas y fotometría, se avanza ahora hacia la presentación de las modelizaciones del alumbrado vial.

Dado que el objetivo central de esta Tesis es la mejora de estos sistemas tomando como eje la optimización de los parámetros lumínicos de calidad y la eficiencia energética, resulta indispensable exponer previamente las definiciones y detalles que caracterizan al alumbrado vial, así como también la normativa vigente, detalles de cálculo y diseño, junto con las nociones básicas para su evaluación (medición).

Como se viene planteando, la caracterización de las propiedades reflectivas de los compuestos asfálticos y su interacción con las fuentes luminosas de nueva tecnología (lámparas de Sodio Compactas - "sodio blanco"- y leds) conforman el núcleo de esta investigación. Se postula entonces que, para poder implementar una metodología de trabajo que permita cuantificar en forma precisa las características reflectivas de las calzadas (tablar) y analizar su vínculo con la eficiencia energética de la instalación, es indispensable conocer antes los fundamentos del alumbrado vial.

\section{Alumbrado vial. Definiciones}

El objetivo de una instalación de alumbrado vial es proporcionar las condiciones visuales necesarias para un tránsito seguro y confortable, facilitando el reconocimiento de la calzada, de los obstáculos, accesos y cruces, de modo de lograr un desplazamiento nocturno rápido y con mínimo riesgo. ${ }^{1}$

Los objetivos anteriores deben, además, alcanzarse con un mínimo costo total de la instalación de alumbrado que contempla, por un lado, el consumo de energía de las luminarias y, por otro, los costos vinculados a la instalación: costo inicial, montaje, mantenimiento, etc. ${ }^{2}$

\section{2- Calidad del alumbrado}

Antes de detallar los métodos o técnicas (Luminancia / Iluminancia) que se emplean en el cálculo o evaluación de una instalación de alumbrado vial, resulta conveniente definir los factores que califican el alumbrado de una vía de tránsito: ${ }^{3}$

\footnotetext{
${ }^{1} \mathrm{CIE}$, Calculation and measurement of luminance and illuminance in road lighting, Publicación 30-2 - TC 4.6, Francia, 1982; Instituto Argentino de Racionalización de Materiales, IRAM AADL J 2022-2, Alumbrado Público, Vías de Tránsito - Clasificación y niveles de iluminación, Buenos Aires, 2009.

${ }^{2}$ Instituto Argentino de Racionalización de Materiales, 2009, op. cit.; De Boer, J., Cohu, M., Schreuder, D., Public Lighting. Philips technical Library, Países Bajos, 1967.

${ }^{3}$ Se siguen las definiciones indicadas en: Instituto Argentino de Racionalización de Materiales, 2009, op. cit.
} 
- Nivel medio del alumbrado.

- Uniformidades.

- Control del deslumbramiento.

\subsection{1- Nivel medio del alumbrado}

Como se comprende, es imposible lograr en una instalación niveles comparables a los obtenidos con luz diurna, los cuales asegurarían las mejores condiciones de visión. Sin embargo, numerosos estudios han permitido obtener valores de iluminación que, con un costo racional de la instalación, permiten alcanzar condiciones de percepción adecuadas. ${ }^{4}$ Dichos niveles se encuentran especificados en las distintas normas o recomendaciones, internacionales o nacionales, expresándose como valores promedios, sobre una determinada área de cálculo o medición. ${ }^{5}$

Es importante destacar que la mayoría de las investigaciones realizadas ${ }^{6}$ concluyen que superar en exceso estos niveles recomendados, no trae aparejado un sustancial incremento en la seguridad en el tránsito, aunque sí aumenta notoriamente el costo de la instalación. En consecuencia, en alumbrado correctamente diseñado se deberá cuidar de cumplir con los niveles medios especificados, sin superarlos excesivamente.

\subsection{2- Uniformidades}

Un adecuado nivel medio no asegura por si sólo las mejores condiciones perceptivas. A pesar de cumplir con este requisito, pueden presentarse sobre el camino zonas con valores locales bajos (sectores oscuros que, desde el punto de vista del conductor, se perciben como manchas o franjas), que pueden enmascarar los obstáculos allí presentes, reduciendo la seguridad en el tránsito. Es por ello que la instalación debe asegurar una cierta relación entre los niveles locales mínimos, máximos y el nivel promedio; estas relaciones se conocen como uniformidades.

Las uniformidades son tan o más importantes que el nivel medio en la calidad del alumbrado. Piénsese, por ejemplo, en conducir por una ruta no iluminada una noche con luna llena. Si bien el nivel medio será muy bajo, la alta uniformidad producida por la "luz lunar", permitiría reconocer bastante bien los límites del camino e incluso detectar ciertos obstáculos.

\subsection{3-Deslumbramiento}

Se conoce con este nombre al efecto que producen fuentes luminosas ubicadas fuera del campo de visión, cuyos rayos luminosos inciden indirectamente en los ojos del observador.

\footnotetext{
${ }^{4}$ De Boer, J., Cohu, M., Schreuder, D., Public Lighting, op. cit.; De Boer, J., Van Bommel, W., Road Lighting, Philips technical Library, Países Bajos, 1980; Illuminating Enineering Society (IES), The Lighting Handbook, 9na. edición, McGraw-Hill, Estados Unidos, 2011; van Bommel, W., Road Lighting - Fundamentals, Technology and Apllication, Springer, 2015.

${ }^{5}$ Por ejemplo en: Instituto Argentino de Racionalización de Materiales, 2009, op. cit.; CEN/TR 13201-1 Technical Report, Road Lighting - Part1: Selection of lighting classes, Bruselas, 2004.

${ }^{6}$ De Boer, J., Van Bommel, W., Road Lighting, op. cit.; van Bommel, W., Road Lighting..., op. cit.; Wordenweber, B., Boyce, P., et al, Automotive Lighting and Human Vision, Springer, 2007.
} 


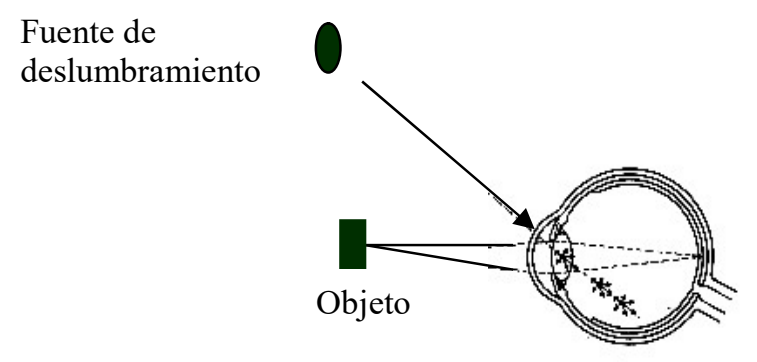

Figura 38. Deslumbramiento. ${ }^{7}$

En lo que respecta al alumbrado vial, son las propias luminarias de la instalación las que podrían llegar a producir deslumbramiento. En un caso extremo, hay riesgo de que la visión quede totalmente "velada" por la mencionada fuente, fenómeno que se conoce como deslumbramiento perturbador. Otro tipo, conocido como deslumbramiento molesto, se produce cuando el conductor recibe en forma constante estos rayos no deseados de una intensidad tal que, aunque no producen efectos perturbadores, reducen el confort en el manejo. ${ }^{8} \mathrm{Si}$ estas condiciones persisten pueden incluso disminuir las aptitudes visuales. ${ }^{9}$

El deslumbramiento es, entonces, el tercer aspecto importante en la calidad del alumbrado. Este debe ser adecuadamente cuantificado y limitado, lo que se logra, en líneas generales, controlando las emisiones de las luminarias para ángulos verticales elevados (cercanos a $\left.90^{\circ}\right){ }^{10}$

\section{3- Condiciones de visión en rutas. La técnica de Luminancia}

Como se abordó en el capítulo anterior, una superficie es visible debido a que la luz que se refleja en ella es detectada por el ojo del observador. Obviamente, si dicha superficie se encuentra muy iluminada será por lo general visible, dependiendo de sus propiedades reflectivas. Esto último (altos niveles de iluminación) no se da, como vimos, en alumbrado vial, donde los objetivos son obtener niveles de visibilidad adecuados al mínimo costo.

\footnotetext{
${ }^{7}$ Imagen tomada de: De Boer, J., Van Bommel, W., Road Lighting, op. cit.

${ }^{8}$ En la bibliografía europea, se alude al término psicológico para el deslumbramiento molesto y fisiológico para el perturbador. En el texto se siguen las definiciones dadas por la norma nacional IRAM.

${ }^{9}$ Instituto Argentino de Racionalización de Materiales, 2009, op. cit.; CIE, International Lighting Vocabulary, publicación 017/E ILV, Austria, 2011; CIE, Discomfort Glare in the Interior Working Environment, publicación 55, Francia, 1983; Hopkinson, R. G., Discomfort glare in lighted streets, Transactions of the IES, 5(1), Londres, 1940; Holladay, L., "The fundamentals of glare and visibility", Journal of the Optical Society of America, 12(4), 1926.

${ }^{10} \mathrm{CIE}$, Calculation and measurement of..., 1982, op. cit.; Instituto Argentino de Racionalización de Materiales, 2009, op. cit.
} 
Erbay, ${ }^{11}$ Sorensen ${ }^{12}$ entre otros, han demostrado que las mejores condiciones de visión en rutas con sistemas de alumbrado artificial, se logran cuando los obstáculos son vistos como siluetas oscuras contra un fondo más brillante, que es la propia calzada. Lo anterior indica que una instalación de alumbrado vial solo puede juzgarse a partir de la distribución de luminancias (L) sobre la calzada: luz reflejada por el camino, que puede asimilarse al concepto perceptivo de "brillo". Como ya se explicó antes, la luminancia es una de las magnitudes (junto con la Iluminancia) que pondera la sensación visual y que permite establecer o definir niveles o límites de visibilidad. Esta permite observar que, cuando dos fuentes de la misma intensidad luminosa con áreas de distinto tamaño, se miran sucesivamente, la menor se percibe como más "brillante". Aparece así la noción de "brillo", muy ligada a la percepción: recortados sobre un mismo fondo, un objeto "brillante" será más fácil de detectar que otro calificado como "opaco".

Como se verá en detalle en el próximo capítulo, la luminancia sobre la calzada depende de tres grandes dominios:

a) Geometría de la instalación.

b) Luz emitida por las luminarias.

c) Propiedades reflectivas de la carpeta de rodamiento.

\subsection{1- Geometría de la instalación}

La posición y cantidad de luminarias por columna, su altura de montaje, la separación entre luminarias, etc. son aspectos que permiten obtener las coordenadas angulares necesarias para efectuar el cálculo de las luminancias en los distintos puntos del área de evaluación. Puede que algunos de estos parámetros se encuentren definidos antes de iniciar el proyecto, mientras que otros surgirán como resultado del proceso de optimización de la instalación.

En los diseños por luminancia es de fundamental importancia la posición del observador, ya que, las propiedades reflectivas de la superficie de la calzada dependen fuertemente de la misma. Por ello, esta ubicación ha sido estandarizada, teniendo en cuenta las posiciones y condiciones de visión que se dan en rutas. ${ }^{13}$

El observador debe ubicarse, para el cálculo y evaluación de instalaciones por la técnica de luminancia, 60 metros antes de la primera línea de luminarias incluidas dentro del área de evaluación. En cuanto a la posición transversal, ésta debe ser a un cuarto del ancho total de la calzada para la obtención de los niveles medios de luminancia y en el centro de cada carril para la determinación de las uniformidades longitudinales. El ángulo de visión estandarizado es de $1^{\circ}\left(\alpha=1^{\circ}\right)$, por lo que la altura del observador con respecto a la calzada, debe ser de 1,5 metros.

\footnotetext{
${ }^{11}$ Erbay, A., Atlas of the reflection properties of road surfaces, ILTUB, Berlin, 1974a.

${ }^{12}$ Sørensen K., Road Surface reflection data. Lysteknisk Laboratorium, The Danish illuminating Engineering Laboratory. Lyngby, Dinamarca, Reporte N 10, 1975.

${ }^{13}$ CIE, Calculation and measurement of..., 1982, op. cit.; Instituto Argentino de Racionalización de Materiales, 2009, op. cit.
} 


\subsection{2- Luminaria}

A la luminaria le cabe la importante función de distribuir el flujo luminoso de la lámpara sobre el camino. Fotométricamente, será mejor aquella luminaria que permita máximas separaciones entre columnas, con menor altura de montaje, cumpliendo lógicamente con los niveles y uniformidades requeridos. No menos importante es la función de proteger tanto a la lámpara como a los equipos auxiliares de la misma. Su hermeticidad, estanquidad y resistencia mecánica son relevantes puesto que de alguna manera determinan la vida útil de la instalación.

Debe evaluarse además la robustez del sistema óptico: una luminaria puede poseer excelentes características fotométricas, pero si su sistema de reflectores es excesivamente débil, es posible que sus buenas aptitudes de distribución de la luz, se pierdan a las pocas horas de uso.

Los factores anteriores deben evaluarse en forma conjunta con el precio del artefacto, en búsqueda de una solución técnico económica global, que incluya los gastos que ocasionará el mantenimiento, reemplazos y la posibilidad de fallas.

\subsection{3- Propiedades reflectivas de los pavimentos}

La superficie de la calzada es el medio reflectivo que genera las luminancias vistas por el observador (conductor). Sus propiedades físicas y químicas (granulometría, tipo de piedra en concretos asfálticos, aglutinante, técnica de aplicación, etc.) determinan el modo en que la luz, proveniente de las luminarias, se reflejará. La reflexión en pavimentos dista mucho de ser difusa, por el contrario, sus formas son variables y extremadamente dependientes de los ángulos de observación e iluminación. Por estos motivos, su caracterización es compleja y será objeto de estudio detallado en el próximo capítulo.

\section{4- La técnica de iluminancia}

En ciertas situaciones, las condiciones de visión se apartan de las adoptadas en la técnica de luminancia. Por ejemplo, en una calle urbana, con tránsito trabado, vehículos estacionados e intensa presencia de peatones, no puede considerarse válido que el observador (conductor) fije su visión sobre una zona situada a 60 metros de distancia. Más aún, no puede suponerse que la visión se mantenga fija en un cierto ángulo. Dado que, como vimos, la luminancia depende fuertemente del ángulo de observación, lo anterior implica que la técnica de luminancia será, cuanto menos, de muy difícil aplicación.

En casos como los anteriormente mencionados, y a pesar que la luminancia es el parámetro apropiado para evaluar lo que el ojo del conductor percibe, se opta por trabajar con la luz que llega a la superficie del camino en lugar de la luz reflejada por él: la iluminancia.

Tal como lo explicamos, se trata de una magnitud que mide la cantidad de luz por unidad de superficie que recibe un cuerpo. Esta se especifica sobre un determinado plano tomado como 
referencia, por lo que es usual hablar de la iluminancia sobre el piso, sobre una pared y en un plano horizontal a determinada altura con respecto al suelo.

La forma en que se aplica se denomina "técnica de iluminancia". En ella sólo se tiene en cuenta el flujo luminoso por unidad de superficie (la iluminancia, E) de la calzada, que puede calcularse a partir de:

$$
E=\frac{I(C, \gamma)}{h^{2}} \cos ^{3}(\gamma)
$$

Puede pensarse a la técnica de iluminancia como una simplificación de la de luminancia, válida para aquellas situaciones en las que la aplicación de la última sea excesivamente compleja. Los diseños y evaluaciones por la técnica de iluminancia son mucho más simples. En efecto, como se deduce de lo dicho, la iluminancia sobre la superficie del camino depende de la geometría de la instalación y de la luz emitida por las luminarias, lo que evita tener que caracterizar a las propiedades reflectivas de los pavimentos.

\section{5- Valores recomendados}

En nuestro país, la norma vigente para el alumbrado vial es la IRAM AADL J 2022-2 (2009), fue aprobada en originalmente en 1995 y prescribe la aplicación tanto de la técnica de luminancia como la de iluminancia, dependiendo de las características de la vía de tránsito a iluminar. Esta combinación de ambas técnicas es una novedad, puesto que la mayoría de las normas y recomendaciones internacionales adoptan sólo una, cualquiera sea la calzada (por lo general, la técnica de luminancia). ${ }^{14}$

En este texto, la técnica de iluminancia se prescribe para el caso de calles en las cuales el tránsito es lento o moderado (velocidades menores a $60 \mathrm{~km} / \mathrm{h}$ ), como son las arterias comerciales, avenidas secundarias, calles colectoras o residenciales. La técnica de luminancia se reserva para autopistas y rutas con tránsito rápido o muy rápido (velocidades mayores a $80 \mathrm{~km} / \mathrm{h}$ ).

Los valores recomendados se obtienen a partir de la clasificación de la arteria. Para ello, la norma establece una tipificación en seis grupos (Tipo "A", "B", "C", "D", "E" y "F"), dependiendo del carácter del tránsito. Para las calzadas "A" y "B", se prescribe la técnica de luminancia, mientras que para las "D", "E" y "F", la de iluminancia. Para las calles clasificadas como tipo " $\mathrm{C}$ ", la mencionada norma indica que el proyectista debe decidir la técnica más adecuada a emplear, por lo que se disponen valores recomendados para ambas técnicas.

\footnotetext{
14 Un completo resumen de la normativa internacional vigente puede encontrarse en: van Bommel, W., Road Lighting..., op. cit.
} 
La tabla siguiente resume los valores recomendados en IRAM AADL J 2022-2 (2009), cuando debe aplicarse la técnica de luminancia:

\begin{tabular}{|c|c|c|c|c|c|}
\hline CLASE & $\begin{array}{c}\text { Luminancias } \\
\text { promedio } \\
\text { iniciales } \\
\text { (cd/m2) }\end{array}$ & $\begin{array}{c}\text { Uniformidad } \\
\text { Uo }\end{array}$ & $\begin{array}{c}\text { Uniformidad } \\
\text { UI }\end{array}$ & $\begin{array}{c}\text { TI } \\
\mathbf{( \% )}\end{array}$ & G \\
\hline A & 2,7 & 0,4 & 0,7 & 10 & 6 \\
\hline B1 & 2,0 & 0,4 & 0,6 & 20 & 5 \\
\hline B2 & 1,3 & 0,4 & 0,6 & 15 & 6 \\
\hline C & 2,7 & 0,4 & 0,6 & 15 & 6 \\
\hline
\end{tabular}

Tabla 1. Valores recomendados en IRAM AADL J 2022-2 (2009), para la técnica de luminancia.

$\mathrm{B} 1$ es una ruta clase "B", con entornos iluminados, mientras que B2 es una ruta clase "B" con entornos no iluminados. Los parámetros TI y $G$, especifican el grado de deslumbramiento admitido: TI cuantifica el deslumbramiento perturbador, $\mathrm{G}$ al molesto. La definición de ambos valores fue extraída de la Recomendación CIE 30-2; ${ }^{15}$ las fórmulas y consideraciones para su cálculo se incluyen en la IRAM AADL J 2022-2. ${ }^{16}$

En cuanto a las uniformidades, en la norma se especifican dos: la uniformidad general Uo, y la uniformidad longitudinal Ul. La primera remite a la relación entre el valor mínimo de luminancia encontrado en la zona de evaluación y la luminancia media:

$$
\text { Uo = Lmín / Lmed }
$$

La uniformidad longitudinal se define como la relación entre las luminancias mínima y máxima halladas en la línea central del carril de observación:

$$
\text { Ul = Lmín / Lmáx }
$$

Este valor debe calcularse o evaluarse para todos los carriles de la calzada, debiendo superar siempre el valor indicado en la tabla.

En cuanto a la técnica de iluminancia, los valores especificados en IRAM AADL J 2022-2 17 son:

\begin{tabular}{|c|c|c|c|c|}
\hline CLASE & $\begin{array}{c}\text { Iluminancia } \\
\text { media inicial } \\
(\mathbf{l x})\end{array}$ & $\begin{array}{c}\text { Uniformidad } \\
\mathbf{G 1}\end{array}$ & $\begin{array}{c}\text { Uniformidad } \\
\mathbf{G 2}\end{array}$ & $\begin{array}{c}\text { Grado mínimo de } \\
\text { apantallamiento de } \\
\text { la luminaria }\end{array}$ \\
\hline $\mathrm{C}$ & 40 & $1 / 2$ & $1 / 4$ & Apantallado \\
\hline $\mathrm{D}$ & 27 & $1 / 3$ & $1 / 6$ & Semiapantallado \\
\hline $\mathrm{E}$ & 16 & $1 / 4$ & $1 / 8$ & Semiapantallado \\
\hline $\mathrm{F}$ & 10 & $1 / 4$ & $1 / 8$ & No apantallado \\
\hline
\end{tabular}

${ }^{15} \mathrm{CIE}$, Calculation and measurement of ..., 1982, op. cit.

${ }^{16}$ Instituto Argentino de Racionalización de Materiales, 2009, op. cit.

${ }^{17}$ Instituto Argentino de Racionalización de Materiales, 2009, op. cit. 
Tabla 2. Valores recomendados en IRAM AADL J 2022-2 (2009), para la técnica de iluminancia.

Las uniformidades establecidas son dos: G1 y G2. La primera se define como la relación entre la iluminancia mínima encontrada en el área de evaluación y el valor promedio:

$$
\text { G1 = Emín/Emed }
$$

G2 se manifiesta como la relación entre las iluminancias mínima y máxima halladas en el área de evaluación:

$$
\text { G2 = Emín/Emáx }
$$

En cuanto al control del deslumbramiento, se especifica el grado mínimo de apantallamiento que debe poseer la luminaria para poder emplearse, según lo establece la IRAM AADL J 2022-1 (2012).

\section{Evaluación de instalaciones de alumbrado vial}

Para la medición de los valores de iluminancia o luminancia en una instalación del alumbrado vial, la norma IRAM AADL J 2022-2 establece un área de evaluación y la define como un "tramo recto de calzada comprendido entre dos soportes de luminarias contiguos". 18 Dicha área se divide en diez puntos en sentido longitudinal y cinco por cada carril, en dirección transversal. Los valores de iluminancia o luminancia se miden en cada uno de estos puntos comprendidos dentro del área de evaluación.

\section{1- Medición de iluminancias}

El área especificada para evaluaciones de iluminancias se muestra en la figura 39:

${ }^{18}$ Ibídem. 


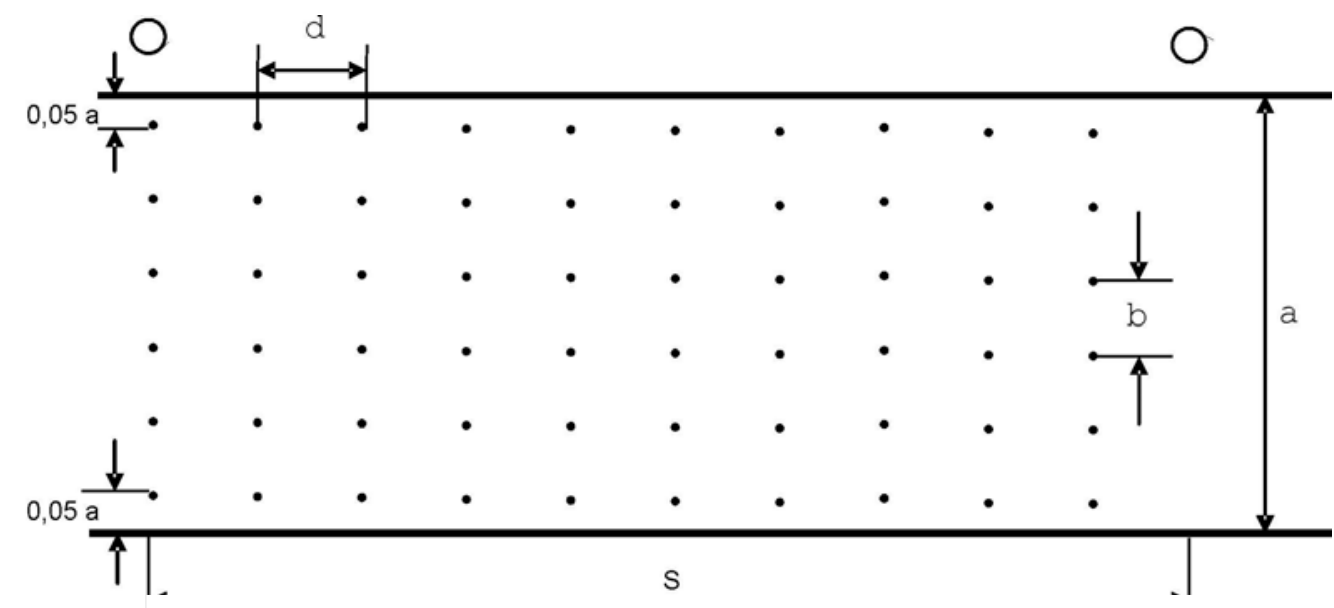

a el ancho de calzada, en metros;

d la distancia longitudinal (en metros), entre los puntos de evaluación $\mathrm{d}=\mathrm{S} / \mathrm{N}$;

$\mathrm{N}$ el número entero, que puede tomar los valores siguientes:

$\mathrm{N}=10$ para $\mathrm{S} \leq 50 \mathrm{~m}$

$\mathrm{N}=$ menor número entero que permite una $\mathrm{d} \leq 5 \mathrm{~m}$ para $\mathrm{S}>50 \mathrm{~m}$

$$
\mathrm{b}=0,9 \cdot \mathrm{a} / \mathrm{M}
$$

b la distancia transversal (en metros), entre los puntos de evaluación;

Figura 39. Área de evaluación para el cálculo de iluminancia. ${ }^{19}$

Los valores de iluminancia, en cada uno de los puntos mencionados, se miden con un luxímetro teniendo en cuenta las siguientes precauciones:

- El instrumento tiene que ubicarse de modo tal de medir iluminancias horizontales.

- El luxímetro deberá estar correctamente calibrado, tener filtro de adaptación a la curva V $\lambda$ y a la ley coseno, de modo de mantener acotados sus errores. En este sentido, la calibración del instrumento debe realizarse anual o a lo sumo bianualmente, de acuerdo a la recomendación de fabricantes y organismos de control. $^{20}$ La calibración puede ser realizada por el propio fabricante o por un laboratorio capacitado.

\footnotetext{
${ }^{19}$ Imagen extraída de: Instituto Argentino de Racionalización de Materiales, 2009, op. cit.

20 "Instruments for Photometry", LMT Lichtmesstechnik GMB, [en línea] URL: http://www.lmtberlin.de/en/ophoto.html [Consulta: 2 de abril de 2019]; Everfine [en línea] URL: http://www.everfine.net/ [Consulta: 2 de abril de 2019]; Superintendencia de Riesgos del Trabajo. Higiene y seguridad en el trabajo. Protocolo para la Medición de la Iluminación en el Ambiente Laboral. Resolución 84/2012. Ministerio de Justicia y Derechos Humanos. Presidencia de la Nación [en línea] URL: http://servicios.infoleg.gob.ar/infolegInternet/anexos/190000-194999/193616/norma.htm [Consulta: 2 de abril de 2019].
} 
- En el caso de instalaciones nuevas, deberá cuidarse que las lámparas se hayan estabilizado, con al menos 200 horas de uso, para que los valores medidos sean realmente representativos de la situación real.

Cabe destacar que, como en toda medición fotométrica, tendrá que procurarse no excluir las fuentes luminosas que no deben medirse, y evitar las sombras producidas por quienes operan el instrumento.

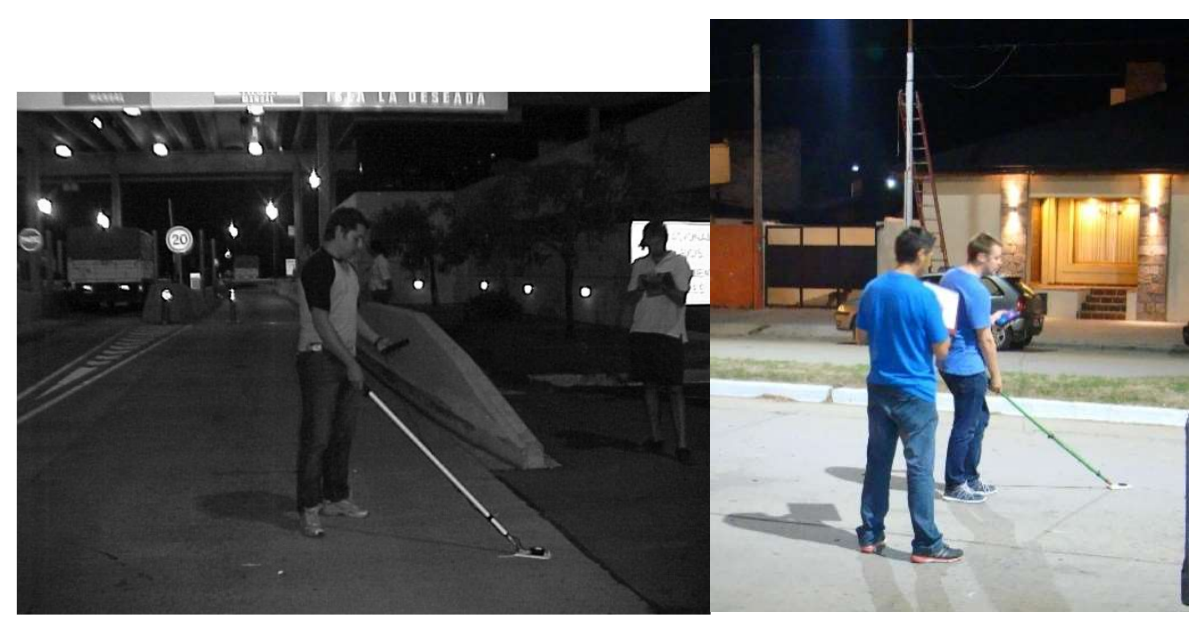

Figura 40. Aspectos de mediciones de iluminancias en calles (fotografías del autor, evaluación de un puesto de peaje -izq.-, medición en calles -der.-).

\section{2- Medición de luminancias}

La medición de luminancias es un poco más compleja que la de iluminancias. Para esto se utiliza un luminancímetro, compuesto por un fotodetector y un sistema óptico que permite enfocar pequeñas porciones del pavimento desde el punto de observación (que se encuentra situado a 60 metros del área de evaluación).

El área de evaluación estandarizada difiere de la empleada en mediciones de iluminancia, estableciéndose una partición por carril de circulación (figura 41). 


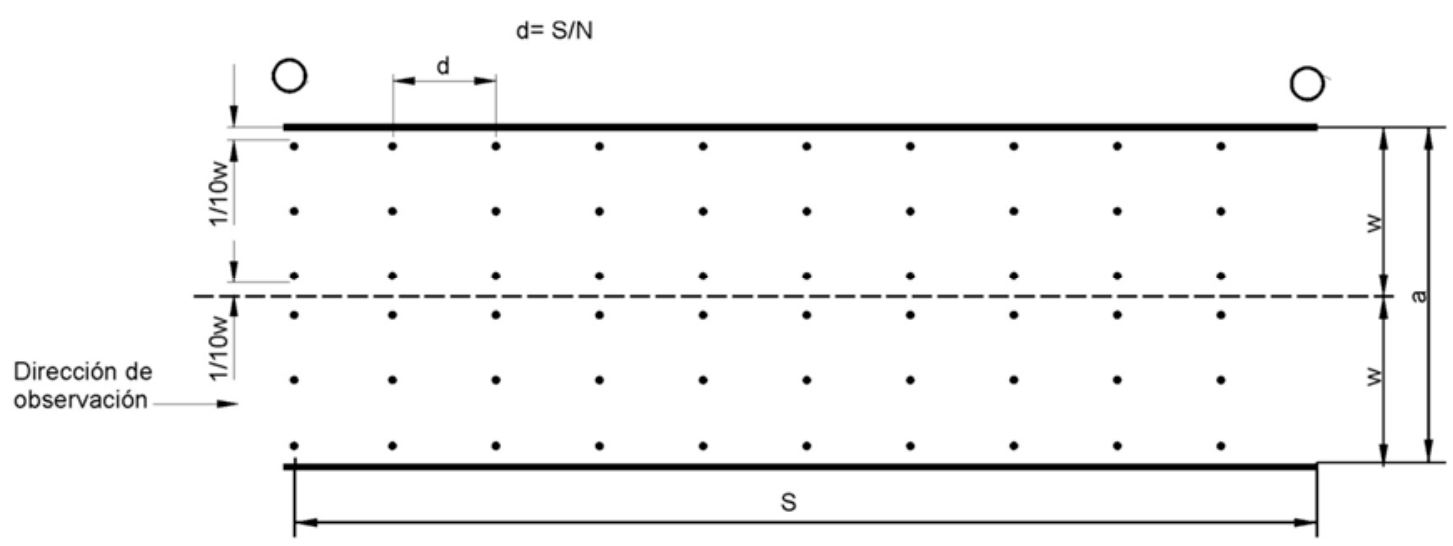

a el ancho de la calzada;
w el ancho del carril;
S el espaciamiento entre las luminarias;
d la distancia longitudinal entre los puntos de evaluación;
N el número entero que puede tomar los valores siguientes:
$\mathrm{N}=10$, para $\mathrm{S}<50 \mathrm{~m}$.
$\mathrm{N}=$ menor entero que permite una $\mathrm{d}<5 \mathrm{~m}$, para $\mathrm{S}>50 \mathrm{~m}$

Figura 41. Área de evaluación para mediciones de luminancia. ${ }^{21}$

Asimismo, en las mediciones debe respetarse la dirección de observación coincidente con el sentido del tránsito, por ejemplo:

a) Dos carriles por sentido con separador central

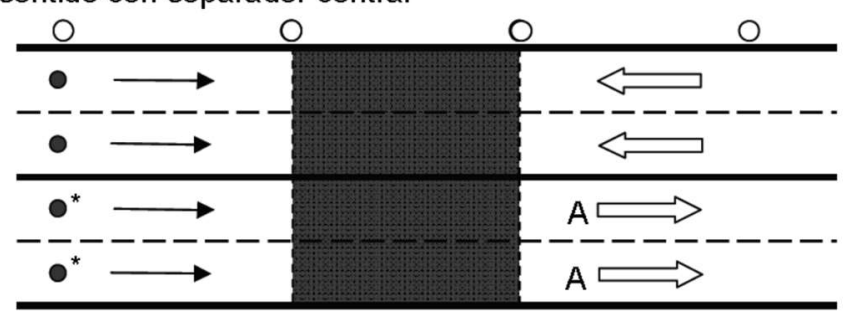

Figura 42. Dirección de observación y sentido del tránsito. ${ }^{22}$

En la medición, el operador enfoca con el luminancímetro el punto a evaluar, con un ángulo vertical de aproximadamente $1^{\circ}$. Esto lo logra, desde la posición de observación, a $60 \mathrm{~m}$ de la primera línea de puntos de medición, ubicando el luminancímetro a 1,50 $\mathrm{m}$ de altura.

\footnotetext{
${ }^{21}$ Imagen extraída de: Instituto Argentino de Racionalización de Materiales, 2009, op. cit.

${ }^{22}$ Ibídem.
} 

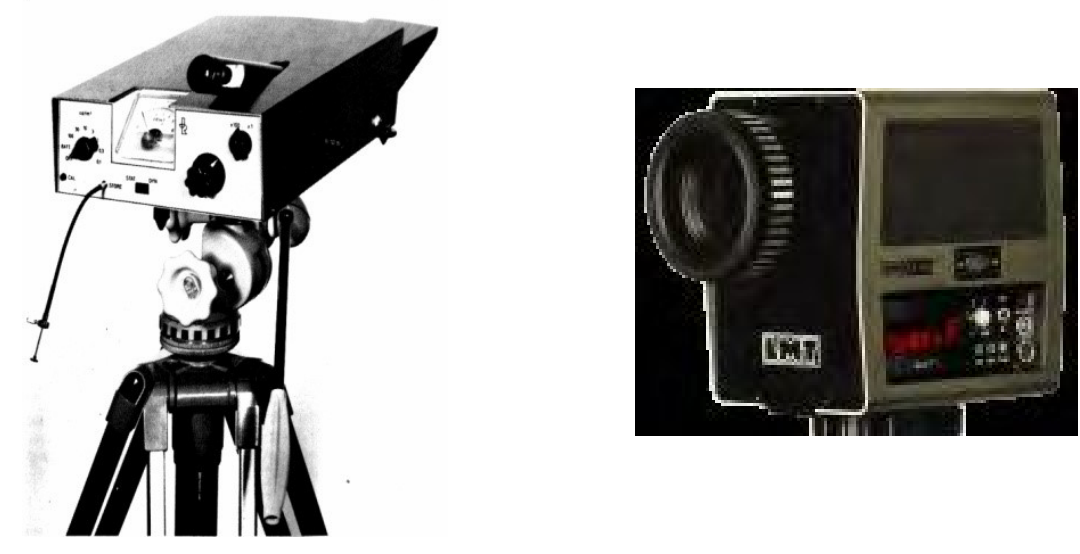

Figura 43. Luminancímetros utilizados en mediciones de luminancia en rutas. Instrumento analógico empleado en los ${ }^{~} 80 .^{23}$ Instrumento digital usado en el LAL. ${ }^{24}$

Las precauciones en este tipo de mediciones son similares a las tenidas en el caso de iluminancia, con la salvedad de que la calibración del luminancímetro, su posicionamiento sobre la calzada y su enfoque requieren más cuidados que en el uso de luxímetros. ${ }^{25}$

\footnotetext{
${ }^{23}$ Imagen extraída de: De Boer, J., Van Bommel, W., Road Lighting, op. cit.

${ }^{24}$ Imagen extraída de: "Instruments for Photometry", LMT ..., op. cit.

${ }^{25}$ Bazalar, P, Ixtaina, P., "Alumbrado vial en base al criterio de luminancia. Comentario sobre los errores en una instalación de alumbrado público unilateral real", Revista Luminotecnia, 48, 1995; Bazalar, P., Ixtaina, P., "Seguridad de las personas en rutas de tránsito rápido. Investigaciones en el Laboratorio de la CIC sobre reflectancia lumínica tendientes a mejorar los actuales métodos en uso", Anales del III Congreso Panamericano de Luminotecnia, 1995a.
} 


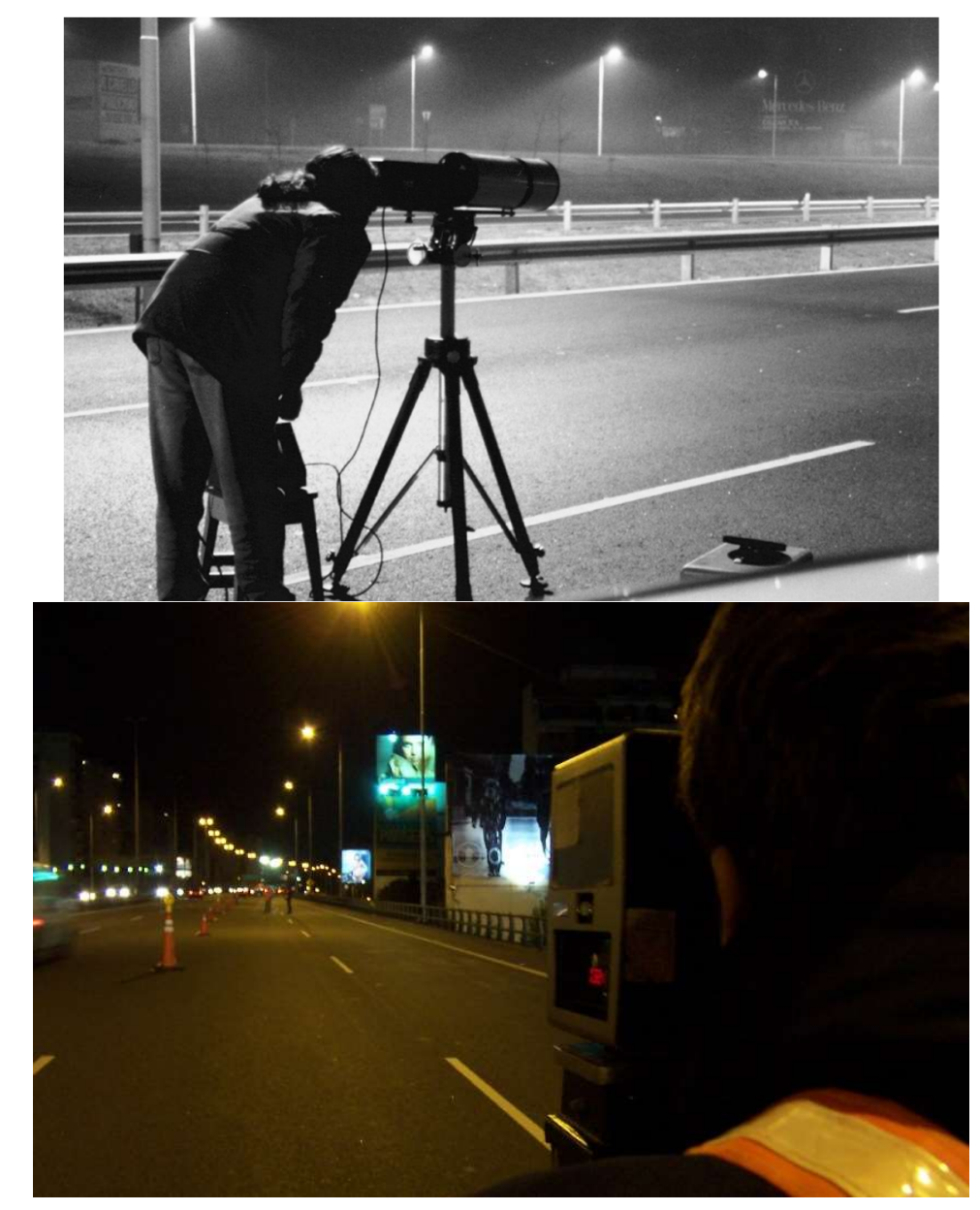

Figura 44. Aspectos de mediciones de luminancias (fotografías del autor).

\section{Recapitulando}

En este apartado se buscó completar las definiciones conceptuales junto con la presentación de una síntesis de las técnicas en uso y la normativa que rige el alumbrado vial. En conjunto con lo expuesto en el capítulo 1, se concluyen las bases necesarias para el abordaje de la línea central de la Tesis que es el estudio y desarrollo de la Técnica de Luminancia, orientado a la caracterización de las propiedades reflectivas de los compuestos asfálticos y su interacción con las fuentes luminosas de nueva tecnología.

Los apartados que siguen profundizarán sobre la Técnica de Luminancia y se centran en los estudios originales realizados por el autor como becario y, posteriomente, investigador en el Laboratorio de Acústica y Luminotecnia de la Comisión de Investigaciones Científicas de la provincia de Buenos Aires. En este punto cabe mencionar la complejidad inherentre a la caracterizacíon de la reflexión en superfices de calzadas. Los estudios originales de las décadas del ${ }^{\prime} 70$ y ${ }^{`} 80^{26}$ establecieron un conjunto de conceptos que dio lugar a una particular

${ }^{26}$ Entre otros, ver: van Bommel, W., Road Lighting..., op. cit.; Erbay, A., Atlas of the reflection..., op. cit.; Sørensen K., Road Surface..., op. cit. 
técnica de aplicación, pero no cerró completamente el tema. En efecto, como se verá a continuación, la reflexión en calzadas está fuertemente influenciada por cuestiones locales (formulación de compuestos asfálticos, materiales, granulometría, técnicas de aplicación, etc.) lo que junto con la complejidad de su medición, no ha permitido conseguir errores suficientemente acotados. Como resultado de esta baja exactitud, es frecuente que las instalaciones no verifiquen en la práctica los parámetros calculados, con la consiguiente complicación técnica y económica que implican las correciones, los cambios de luminarias, los enfoques, las ópticas, etc.

Este hecho se ha verificado en repetidas oportunidades a nivel local ${ }^{27}$ y resolverlo se ha vuelto un tema de interés a nivel internacional (ver como ejemplo las actividades del Comité específico de la Comisión Internacional de Alumbrado $\mathrm{CIE}^{28}$ ).

Las investigaciones originales que se detallarán en los capítulos siguientes abarcan:

- La Técnica de Luminancia. Problemática de la caracterización de calzadas.

- Desarrollo de un método original, bases teóricas e implementación.

- Aplicaciones del Reflectómetro de Calzadas LAL-CIC en la caracterización de pavimentos de la región.

- Estudios sobre el efecto del desgaste del pavimentos en la reflexión.

- Influencia del pavimento en la eficiencia enérgética de la instalción de alumbrado.

- Influencia del espectro de la fuente luminosa.

Los dós últimos ítems se analizan para las nuevas tecnologías de lámparas (LEDs).

\footnotetext{
${ }^{27}$ Bazalar, P, Ixtaina, P., “Alumbrado vial en base...”, op. cit.; Bazalar, P., Ixtaina, P., "Seguridad de las personas...", op. cit.

${ }^{28}$ Rossi, G., "Synthesis of the discussions on 'photometric measurements of Road surfaces", CIE symposium on road surface photometric characteristics - Measurement systems and results. Turin, 2008.

CIE, "Division 4 Technical Committees", Commission Internationale de l'Eclairage (CIE), [en línea] URL: http://cie.co.at/technical-work/division4/technical-committees [Consulta: 2 de abril de 2019].
} 
Capítulo 3 - Caracterización de las propiedades reflectivas de las calzadas 
Si -como dijimos- el foco de esta Tesis está puesto en producir una mejora de los sistemas de alumbrado vial, resulta clave un estudio pormenorizado de la Técnica de Luminancia y con ello, de la reflexión de la luz en el pavimento. Buscamos demostrar la siguiente hipótesis: las propiedades reflectivas de las calzadas dependen fuertemente de factores locales (materiales usados -piedras y aglutinante-), proporciones, granulometría, técnica de construcción y métodos de aplicación y conformación. Esto determina que los estudios realizados en una región difícilmente puedan extrapolarse a otra.

Sobre la base de esta hipótesis, se considera que no es correcto el uso en nuestro medio de datos de reflexión obtenidos a partir de estudios europeos, lo que constituía una práctica usual: los diseños locales de iluminación vial empleaban "formas de reflexión estándares" europeas, obtenidas de estudios realizados en los años ' 70 y ' 80 centralmente en Alemania, Dinamarca y Suecia. Por el contrario, y como se verá a continuación, el eje de la investigación es, a la par de la verificación de la hipótesis, el desarrollo de un nuevo método de trabajo.

En el punto que sigue, se hace referencia al modelado de la instalación de alumbrado vial, desarrollado para la implementación de la Técnica de Luminancia. Esto permitirá caracterizar las propiedades reflectivas de las calzadas a través de una matriz (la tabla-r). Se continua luego con la "técnica tradicional", desarrollada en los `70, y que se basa en la medición de muestras de pavimentos o probetas, el uso de un sistema de descripción y clasificación y la elección de una matriz estándar de acuerdo al tipo de superficie del camino.

Finalmente se comentan los estudios realizados en el Laboratorio de Acústica y Luminotecnia (LAL) que son antecedentes de los trabajos originales realizados por el autor y que se detallan en el capítulo siguiente. Estos trabajos, basados en el análisis de muestras de calzadas de la provincia de Buenos Aires, concluyen como se verá, en un sistema alternativo para la caracterización de calzadas, que sirve a su vez como base para el posterior desarrollo de un equipo de medición.

\section{1.- Coeficiente de luminancia}

Tomando como base el esquema clásico de la figura 45, la luminancia L de una superficie elemental $\Delta \mathrm{S}$ sobre la calzada, se calcula a partir de: ${ }^{1}$

$$
\mathrm{L}=\frac{\mathrm{I}(\mathrm{C}, \gamma)}{\mathrm{h}^{2}} \mathrm{q}(\alpha, \beta, \gamma, \delta) \cos ^{3}(\gamma)
$$

Decimos que "I $(\mathrm{C}, \gamma)$ " es la intensidad luminosa de la luminaria en dirección al punto en el que se calcula la luminancia, $h$ la altura de montaje de la luminaria y $q$ es el coeficiente

\footnotetext{
${ }^{1} \mathrm{CIE}$, Calculation and measurement of luminance and illuminance in road lighting, Publicación 30-2 - TC 4.6, Francia, 1982; De Boer, J., Cohu, M., Schreuder, D., Public Lighting. Philips technical Library, Países Bajos, 1967; De Boer, J., Van Bommel, W., Road Lighting, Philips technical Library, Países Bajos, 1980.
} 
de luminancia del pavimento. El uso de la luminancia sobre la calzada como parámetro de diseño y calidad se suele denominar Técnica de Luminancia. ${ }^{2}$

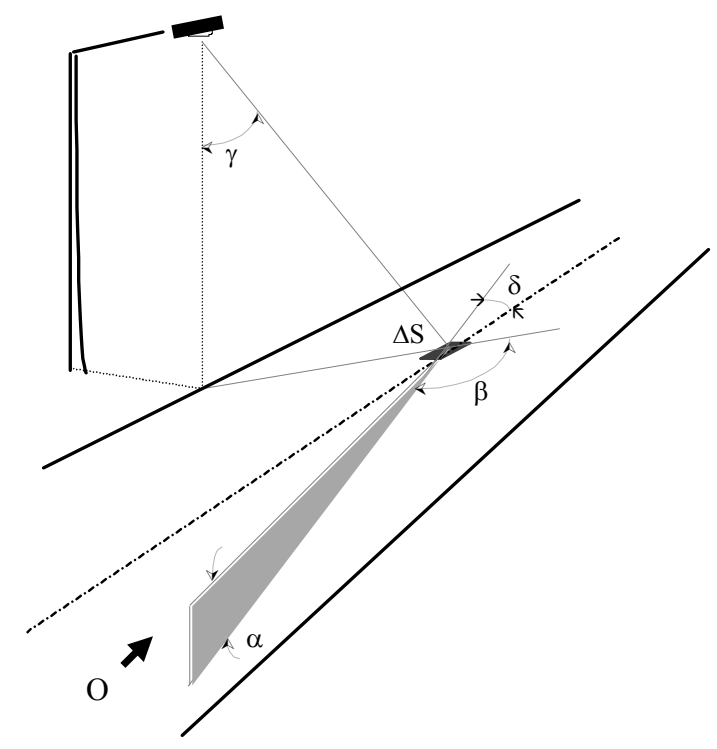

Figura 45. Geometría básica para el análisis de la visión en rutas. ${ }^{3}$

La característica distintiva de la Técnica de Luminancia es que contempla la luz reflejada por la calzada hacia el conductor y por lo tanto requiere conocer las características reflectivas del pavimento. Éstas son cuantificadas por medio del coeficiente de luminancia de la calzada $q$, que depende del material de la superficie del camino: su textura, composición, método de aplicación, tiempo de uso, etc. Lejos de ser una constante de la superficie, su valor se compone en función de las posiciones del observador y de la fuente luminosa con respecto al punto que se considera. En principio es una función de cuatro variables angulares: dos ángulos de observación $(\alpha$ y $\delta)$ y dos de incidencia del rayo luminoso $(\beta \mathrm{y} \gamma)$. Para las condiciones de observación pertinentes a conductores de automóviles en rutas, los ángulos $\alpha$ y $\delta$ no presentan grandes

2 CIE, Calculation and measurement..., 1982, op. cit.; Instituto Argentino de Racionalización de Materiales, IRAM AADL J 2022-2, Alumbrado Público, Vias de Tránsito - Clasificación y niveles de iluminación, Buenos Aires, 2009; Erbay, A., Atlas of the reflection properties of road surfaces, ILTUB, Berlin, 1974a.

${ }^{3}$ Imagen extraída de: CIE, Calculation and measurement..., 1982, op. cit. 
variaciones, por lo que se han adoptado valores fijos para ambos $\left(\alpha=1^{\mathrm{o}}\right.$ y $\left.\delta=0^{\circ}\right)$, de modo tal que puede considerarse a $q$ dependiente solo de $\beta$ y $\gamma .{ }^{4}$

Por limitaciones de los métodos de cálculo en la década de los ' 70 (métodos gráficos, sin auxilio de procesadores ${ }^{5}$ ), cuando se desarrollaron las bases de la Técnica de Luminancia, se estandarizó el uso de los llamados coeficientes de luminancia reducidos " $\mathrm{r}$ ":

$$
r_{(\beta, \tan \gamma)}=q(\beta, \gamma) \cdot \cos ^{3}(\gamma)
$$

Pese a que las limitaciones de cálculo no existen hoy en día, el uso del coeficiente reducido continúa vigente. ${ }^{6}$ De esa forma, la manera práctica de especificar completamente las características de reflexión de la superficie de un pavimento es mediante la llamada "tabla-r", matriz con un formato normalizado, que indica los valores del coeficiente " $r$ " para un conjunto de pares de ángulos de incidencia $\beta$ y $\gamma .{ }^{7}$

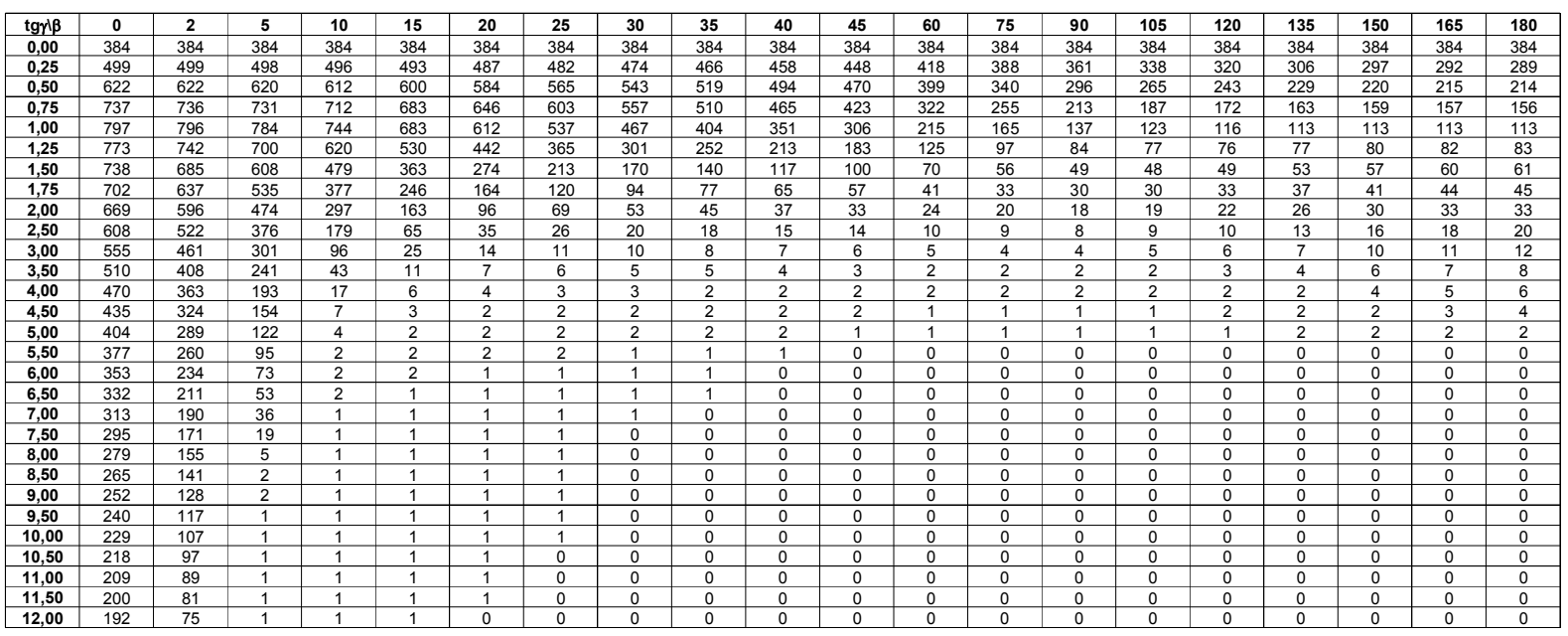

Figura 46. Formato estandarizado para especificar las características del pavimento (Tabla-r). ${ }^{8}$

El punto crítico de la Técnica de Luminancia es cómo obtener las características reflectivas del pavimento real de la instalación (su tabla-r). Sin este dato es prácticamente imposible lograr un buen diseño del sistema de alumbrado. El punto siguiente abordará

\footnotetext{
${ }^{4}$ Instituto Argentino de Racionalización de Materiales, 2009, op. cit.; CIE, Road surface and road marking reflection characteristics, publicación TC 4.24 y 4.25-144, Francia, 2001; Erbay, A., Atlas of the reflection..., op. cit.

5 Erbay, A., Atlas of the reflection..., op. cit.; Sørensen K., Road Surface reflection data. Lysteknisk Laboratorium, The Danish illuminating Engineering Laboratory. Lyngby, Dinamarca, Reporte N 10, 1975. ${ }^{6}$ Instituto Argentino de Racionalización de Materiales, 2009, op. cit.; CIE, Road surface and..., 2001, op. cit.

7 CIE, Calculation and measurement..., 1982, op. cit.; Instituto Argentino de Racionalización de Materiales, 2009, op. cit.

8 CIE, Calculation and measurement..., 1982, op. cit.; Instituto Argentino de Racionalización de Materiales, 2009, op. cit.
} 
justamente las soluciones ensayadas internacionalmente para alcanzar una buena aproximación a la tabla-r de la calzada de la instalación.

\section{2.- Obtención de la tabla-r}

Existen tres caminos para obtener la tabla-r de una calzada: ${ }^{9}$

a.- Medición de la tabla completa a partir de muestras o probetas.

b.- Caracterización del pavimento a partir del uso de un sistema de descripción.

c.- Elección de una matriz estándar a partir de la inspección del tipo de superficie del camino.

\section{1- Medición de probetas}

La medición de muestras o probetas cortadas del camino real puede considerarse el método más exacto, siempre que el fragmento de superficie de la calzada medido en el laboratorio sea realmente representativo de la carpeta asfáltica. Esta tarea se realiza con un equipo especialmente diseñado para tal fin (Reflectómetro de Muestras), que recrea en el laboratorio las condiciones naturales de observación propias de las rutas.

Existen varios modelos de dispositivos para medir estas muestras, que se basan en el mismo procedimiento: primero, una fuente luminosa se abate sobre la muestra, lo que posibilita describir las dos coordenadas angulares $(\beta$ y $\gamma)$. Luego, se aplica el sistema de observación: un luminancímetro que debe enfocarse hacia la muestra asegurando un ángulo de observación $\alpha=1^{\circ}$. A continuación, se ven algunos ejemplos de estos equipos.

La figura 47 muestra un esquema y una imagen del equipo empleado por Philips a mediados de los '80, cuyo rasgo central era que el luminancímetro se movía alrededor de la muestra, describiendo el ángulo $\beta$.

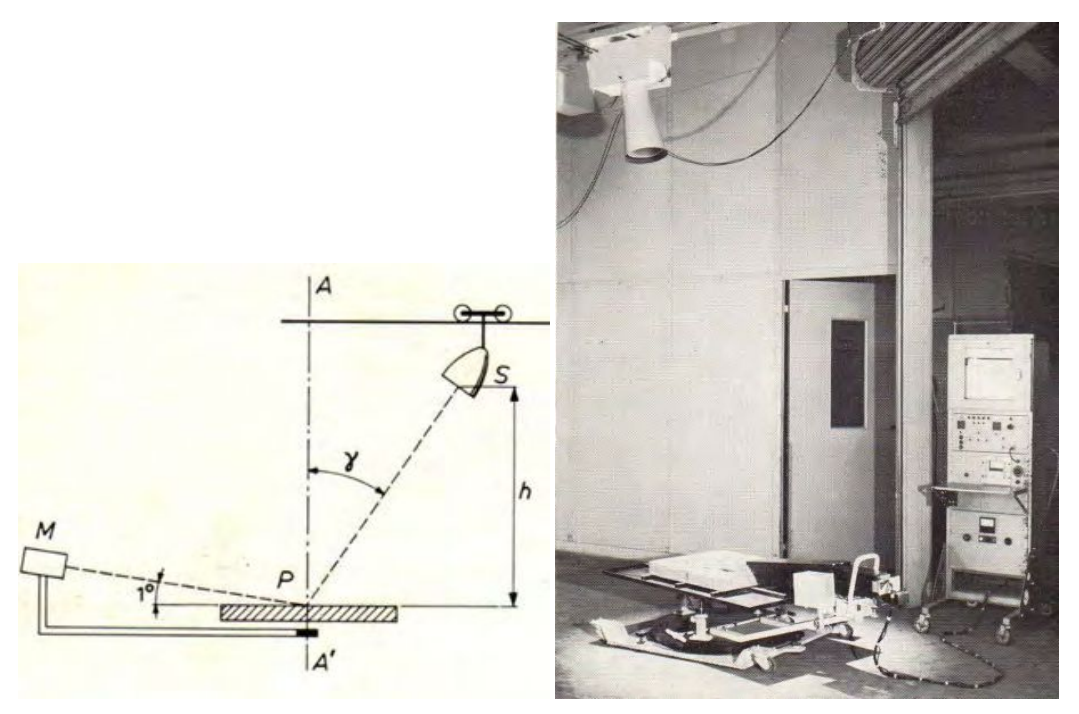

\footnotetext{
${ }^{9}$ CIE, Road surface and..., 2001, op. cit.
} 
Figura 47. Reflectómetro de Muestras. ${ }^{10}$

En la figura 48 se ve el equipo desarrollado en el LAL-CIC para medición de muestras que se aplicó en las primeras investigaciones sobre el tema, que estudiaron pavimentos de la provincia de Buenos Aires. La figura ilustra además muestras usadas en estos análisis, obtenidas de rutas del interior de la provincia en los ' 90.

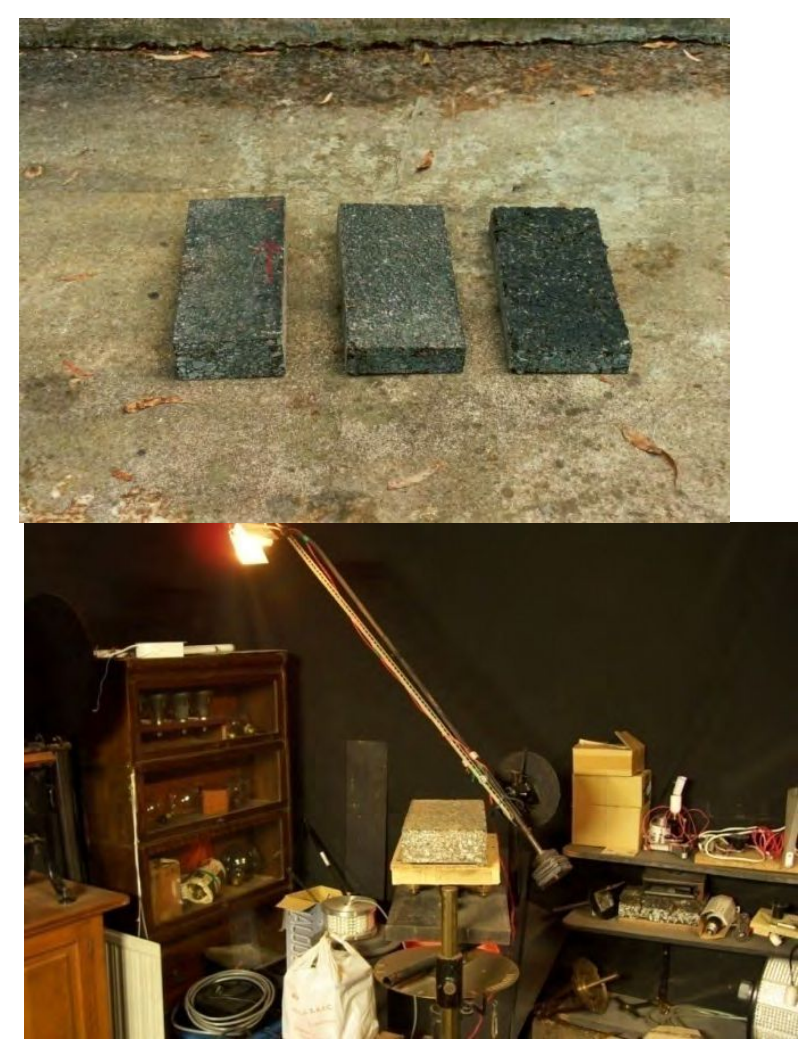

Figura 48. Reflectómetro de Muestras LAL-CIC (fotografías del autor).

Si bien se considera la medición de la tabla completa a partir de probetas como el método más confiable, cabe destacar que la extracción de muestras no siempre reproduce las características del pavimento real de la instalación. El sólo hecho de cortar la carpeta y trasladar la muestra implica muchas veces alteraciones y deformaciones que conducen a errores difíciles de evaluar. Por otra parte, existen dificultades prácticas (como la coordinación de la obra civil con el proyecto de alumbrado, o que la superficie que se mide no está generalmente rodada, etc.) que complican está opción, quedando reservada sólo para fines de investigación. ${ }^{11}$

\section{2- Métodos simplificados: sistemas de descripción}

Las dificultades de la obtención y posterior medición de muestras han motivado la búsqueda de métodos simplificados para obtener la tabla-r. La idea es definir unos pocos

\footnotetext{
${ }^{10}$ Imagen extraída de: De Boer, J., Van Bommel, W., Road Lighting, op. cit.

${ }^{11}$ CIE, Road surface and..., 2001, op. cit.
} 
parámetros, basados en relaciones entre coeficientes de luminancia reducidos de posiciones particulares, que sean suficientemente representativos de las propiedades reflectivas globales de la calzada. Estos parámetros deben además permitir su medición en el lugar (sobre el camino) con algún instrumento. El conjunto de condiciones que caracterizan las propiedades de las calzadas se denominan sistema de descripción. ${ }^{12}$

Sobre esta idea, dos calzadas con propiedades reflectivas similares tendrán análogos elementos de descripción. El método simplificado consiste entonces en medir estos parámetros sobre el pavimento a caracterizar y luego buscar una tabla-r ya completa (a partir de muestras) que contenga parámetros similares. Esta tabla-r será entonces representativa de la calzada de la instalación. Se obtiene así una tabla-r para el cálculo (aproximada) sin necesidad de extraer muestras del camino.

Dado que la tabla-r consta de alrededor de 400 coeficientes de luminancia reducidos, su representación en un sistema de descripción, por unos pocos valores (generalmente relaciones de tales coeficientes) adolecerá de errores y justamente la virtud del sistema de descripción será ajustarse lo mejor posible a las características del pavimento en estudio.

El desarrollo de un buen sistema de descripción fue un tema central al momento de modelar los conceptos de la Técnica de Luminancia y fueron propuestos muchos tipos. Finalmente, la Comisión Internacional de Alumbrado CIE adoptó el sistema de descripción desarrollado por Ahsen Erbay a mediados de los '70. Este constaba de tres parámetros de descripción:

$$
\begin{aligned}
& \mathrm{Q}_{0}=\int_{0}^{\Omega 0} \mathrm{q} \cdot \mathrm{d} \Omega \quad \text { (Coeficiente de luminancia media) } \\
& \mathrm{S} 1=\frac{\mathrm{r}(0,2)}{\mathrm{r}(0,0)} \quad \text { (Factor especular) } \\
& \mathrm{S} 2=\frac{\mathrm{Q}_{0}}{\mathrm{r}(0,0)}
\end{aligned}
$$

El coeficiente de luminancia media se define como la integral de los valores de coeficiente de luminancia sobre el ángulo sólido $(\Omega 0)$ con vértice en el punto de la calzada y que contiene las posibles direcciones de incidencia de la luz. En términos prácticos, se determina con los límites indicados en la figura 49.

\footnotetext{
${ }^{12}$ Erbay, A., Atlas of the reflection..., op. cit.; Sørensen K., Road Surface reflection..., op. cit.
} 


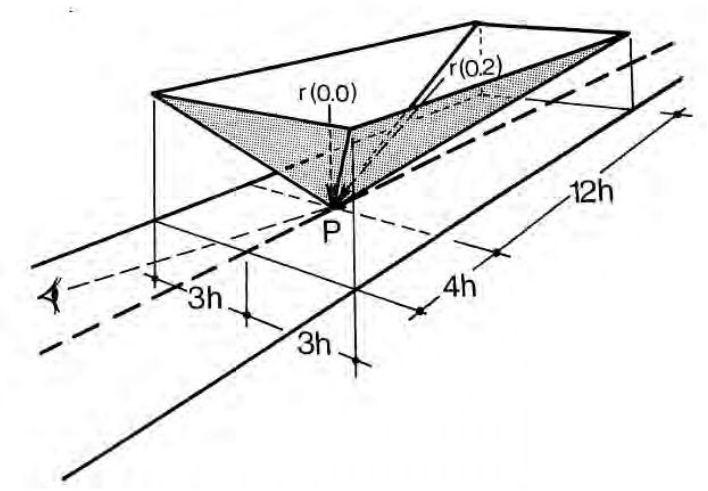

Figura 49. Ángulo sólido $\Omega$ y sus límites. ${ }^{13}$

El coeficiente Qo cuantifica de alguna manera el "grado de claridad" de la calzada y fue normalmente usado como factor de escala. Los coeficientes S1 y S2 fueron los responsables de caracterizar la "forma" de la matriz de reflectancia. ${ }^{14}$

La utilidad del sistema dependía de las posibilidades reales de medir los coeficientes en la calzada y sobre el pavimento real. Lamentablemente, el parámetro Qo incorporó una definición tal que limitó el desarrollo de instrumentos de medición. Si bien se han ensayado prototipos de instrumentos que "integraban" la iluminación de una porción del pavimento, la falta de exactitud de estos equipos limitó la difusión y aplicación del sistema de Erbay. Por ejemplo, el equipo de la figura 50, desarrollado por Philips Holanda en la década del '80, llegó a tener versiones comerciales, adquiridas incluso en nuestro país. Sin embargo, fueron escasos los antecedentes de aplicaciones reales, más allá de las pruebas del instrumento. ${ }^{15}$

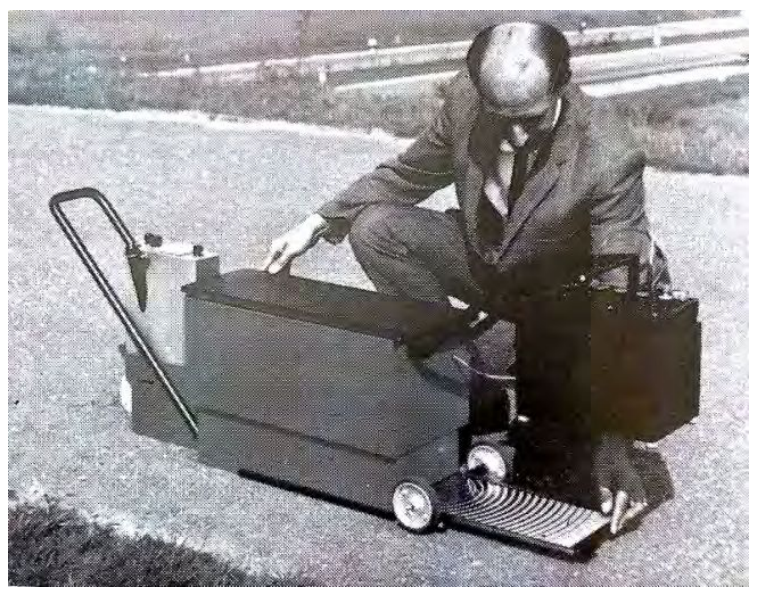

\footnotetext{
${ }^{13}$ Imagen extraída de: De Boer, J., Van Bommel, W., Road Lighting, op. cit.

${ }^{14}$ CIE, Calculation and measurement..., 1982, op. cit.; De Boer, J., Van Bommel, W., Road Lighting, op. cit.

${ }^{15}$ Vidal, P. A., Ixtaina, P. R., "New System of identification of de reflection indicatrices of pavements", note to CIE TC 4-25, Francia, 1996; Vidal, P. A., Ixtaina, P. R., "New method for characterising the reflection properties of road surfaces", CIE $25^{\text {th }}$ Session, San Diego, 2003.
} 
Figura 50. Prototipo para medir los coeficientes S1, S1 y Qo. ${ }^{16}$

Tratando de salvar esta cuestión, Burghout propuso un sistema de descripción, que evitaba la complejidad del coeficiente Qo. ${ }^{17}$ Los parámetros usados fueron:

$$
\begin{aligned}
& \mathrm{P} 1=\frac{\mathrm{r}(0,2)}{\mathrm{r}(0,0)}=\mathrm{S} 1 \\
& \mathrm{P} 2=\frac{\mathrm{r}(90,1)}{\mathrm{r}(0,0)}
\end{aligned}
$$

Aquí ambos valores podían obtenerse en forma simple, pero las inexactitudes del sistema de descripción, según lo evaluado por Burghout en su estudio, aumentaban.

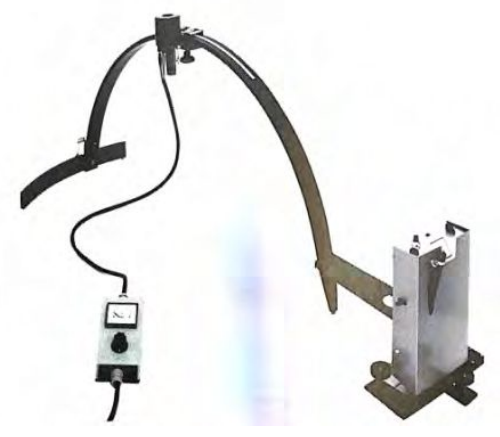

Figura 51. Equipo diseñado por Range para medir los coeficientes de Erbay o Burghout. ${ }^{18}$

\section{3- Métodos simplificados: uso de pavimentos estándar}

En la década del '70, el desarrollo del sistema CIE-Erbay estuvo acompañado por la medición de numerosas muestras de pavimento, en un intento por caracterizar los asfaltos de la región. ${ }^{19}$ Por un lado, el propio Erbay midió y clasificó 240 superficies, principalmente de la zona central de Alemania. Sorensen por su parte, realizó un trabajo similar para Dinamarca y Suecia. En ambos estudios, las tablas-r fueron ordenadas a partir

\footnotetext{
${ }^{16}$ Imagen extraída de: De Boer, J., Van Bommel, W., Road Lighting, op. cit.

${ }^{17}$ Burghout, F., "Two class system for the classification of dry road surfaces with regard to light reflection", CIE Journal 5(1), pp. 22-43, 1986.

${ }^{18}$ Imagen extraída de: Range, H., "Road Reflectometer for the simplified determination of the properties of lighted road surfaces", Lichttechnik, 25, p. 389, 1973.

${ }^{19}$ Erbay, A., Atlas of the reflection..., op. cit.; Sørensen K., Road Surface reflection..., op. cit.; Keitz, H. A. E, Light calculations and measurements, N. V. Philips, Holanda, 1974.
} 
de valores de S1 creciente con la idea (no totalmente demostrada) de que las superficies con S1 bajo se presentan como más "difusas" que las de S1 mayores.

De este modo, la aplicación del sistema de descripción a la base de datos de tablas-r medidas hasta ese momento, permitió realizar una clasificación general de calzadas. El paso siguiente fue la "estandarización" mediante la cual, cuatro tablas-r convenientemente definidas pasarían a representar la totalidad de los pavimentos estudiados. Este primer sistema de clasificación, denominado "serie R", se componía de cuatro pavimentos estándares, denominados: R1, R2, R3 y R4 y cuyas tablas-r se consideraban representativas de cuatro tipos o clases de calzadas contenidas en los "atlas" mencionados. Como ejemplo, la figura 52 muestra los diagramas espaciales de los pavimentos estándares R1 y R4.

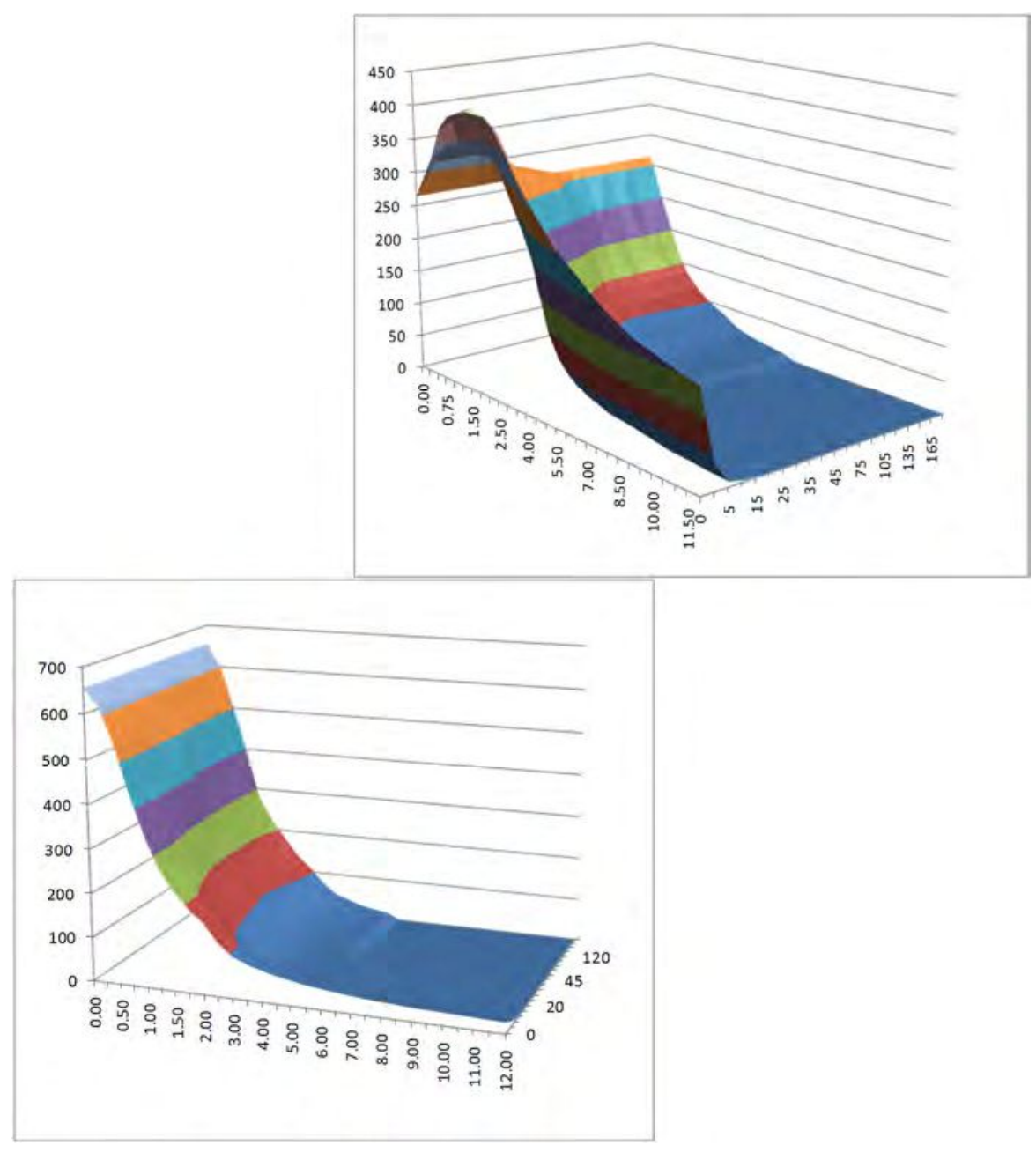

Figura 52. Pavimentos estándares R1 y R4. ${ }^{20}$

\footnotetext{
${ }^{20}$ CIE, Calculation and measurement..., 1982, op. cit.
} 
La figura 52 muestra en un formato más gráfico las tablas-r estandarizadas como R1 y R4. El eje vertical se corresponde con los valores de coeficientes reducidos, en los ejes horizontales se observan valores de ángulos $\beta$ y tg $\gamma$ (ver figura 46).

El estándar R1 se corresponde con una superficie difusa y puede fácilmente asimilarse a un hormigón. Diametralmente opuesto, el R4 representa una reflexión más selectiva, mostrando un pico típico de una superficie conocida como "especular", forma propia de los concretos asfálticos.

La serie R no fue la única propuesta de clasificación de calzadas. En su trabajo, Burghout admite que las inexactitudes propias de la descripción vuelven irrelevantes la excesiva clasificación de calzadas, y propone agruparlas en solo dos clases, a la que les asignó dos pavimentos estándares denominados "Serie C" (CI y CII). ${ }^{21}$ También desarrolló una serie $\mathrm{N}$, aplicable a las calzadas típicas de los países nórdicos. ${ }^{22}$ Las investigaciones en este campo se detuvieron desde fines de los ' 80 , lo que llevó a la CIE a expresar la necesidad de continuar con los estudios. ${ }^{23}$ En Argentina, y sobre la base de la experiencia nacional, la norma IRAM adoptó en su última versión, el sistema $\mathrm{R}$ de clasificación de calzadas. ${ }^{24}$

\section{3.- Aplicación de la clasificación de calzadas}

Como se dijo, el uso de una matriz estándar (opción "c") fue hasta hace unos años, la práctica habitual de diseño de sistemas de alumbrado vial en nuestro medio. Los concretos asfálticos se representaban usando el sistema de clasificación de la serie R, adoptándose por lo general en el cálculo la tabla-r del pavimento R3. Como es sabido, ${ }^{25}$ esta metodología de trabajo constituye una simplificación exagerada, dado que no media proceso alguno de medición, quedando librada la elección de un dato fundamental para el cálculo (la tabla-r) al criterio o a la experiencia del proyectista. Esto eleva notablemente la incertidumbre en la obtención de resultados luminotécnicos: los parámetros de calidad en luminancia proyectados para la calzada no se verifican en la práctica cuando la instalación de alumbrado es evaluada, lo que obliga a procesos del tipo prueba y error. Obviamente lo anterior implica mayores costos y tiempos hasta que las luminarias de la instalación son ajustadas.

La hipótesis que aquí se postula es que la medición "in-situ" de parámetros de descripción, como un camino intermedio, puede asegurar una mejorada exactitud y practicidad y, asimismo, conducir a una mejora en los diseños de instalaciones de alumbrado vial. Sin bien los equipos propuestos para el sistema de Erbay (arco de Range, reflectómetro de De Boer, etc. $)^{26}$ no pudieron conseguir una precisión tal que los hiciera viables, los estudios elaborados para la Tesis indican que el uso de un sistema de

\footnotetext{
${ }^{21}$ Burghout, F., "Two class system for...”, op. cit.

22 De Boer, J., Cohu, M., Schreuder, D., Public Lighting, op. cit.; De Boer, J., Van Bommel, W., Road Lighting, op. cit.

${ }^{23}$ CIE, Road surface and road marking..., 2001, op. cit.

${ }^{24}$ Instituto Argentino de Racionalización de Materiales, 2009, op. cit.

${ }^{25}$ CIE, Road surface and road marking..., 2001, op. cit.; Vidal, P. A., Ixtaina, P. R., "New System of...", op. cit.; Vidal, P. A., Ixtaina, P. R., "New method for characterising...", op. cit.

${ }^{26}$ Instituto Argentino de Racionalización de Materiales, 2009, op. cit.; Range, H., "Road Reflectometer for the...", op. cit.; Keitz, H. A. E, Light calculations and..., op. cit.
} 
descripción mejorado, junto con una técnica de medición adecuada que reduzca los errores, introducen notables progresos en la aplicación. ${ }^{27}$

\section{4.- Sistema de descripción LAL-CIC}

Las dificultades encontradas por los investigadores del LAL al intentar clasificar los pavimentos medidos (provenientes de la provincia de Buenos Aires) dentro del sistema CIE motivaron la orientación de la investigación hacia un sistema de descripción alternativo. En líneas generales, las calzadas medidas poseían índices S1 "bajos", que los clasificaban como "difusos", sin embargo, la inspección de sus tablas mostraba "picos" de reflexión asimilables con superficies más especulares.

Los detalles de esta investigación exceden el objeto de la Tesis, tan solo se destaca que como resultado se obtuvo un nuevo sistema de descripción, denominado Sistema LALCIC, como una alternativa al planteado por Erbay. El estudio fue discutido en la reunión anual del Comité TC 4-25 de la Comisión Internacional de Alumbrado CIE en octubre de $1996^{28}$ y fue posteriormente incorporado como nueva propuesta en la recomendación CIE $144 .^{29}$

El sistema LAL-CIC está compuesto por tres parámetros de descripción: $A_{D}, A_{I 1}$ y $A_{I 2}$, definidos siguiendo la idea de Burghout ${ }^{30}$ de cocientes de valores " $r$ ":

$$
\text { (1) } \mathrm{A}_{\mathrm{D}}=\frac{\mathrm{r}_{(0,0.625)}}{\mathrm{r}_{(0,0)}} \quad \mathrm{A}_{\mathrm{I} 1}=\frac{\mathrm{r}_{(0,3)}}{\mathrm{r}_{(0,0.625)}} \quad \mathrm{A}_{\mathrm{I} 2}=\frac{\mathrm{r}_{(90,1)}}{\mathrm{r}_{(0,0.625)}}
$$

Por un lado, los parámetros del nuevo sistema recogen información desde dos posiciones adicionales originales (pares angulares $\beta$, tg $\gamma 0,3$ y 90,1) que recogen información importante sobre las rugosidades propias de la superficie. ${ }^{31}$ Por otro, al no usar el parámetro Qo, pueden calcularse directamente a partir de mediciones de valores " $r$ ", que eventualmente, pueden realizarse sobre la propia calzada (sin necesidad de obtener muestras). Esto permite sortear las dificultades que presenta la medición del parámetro Qo del sistema de Erbay, que requiere de un dispositivo complejo y poco práctico para su medición "in situ". ${ }^{32}$

Tal como se mencionó anteriormente, los equipos propuestos para la medición "in situ" de los parámetros de descripción (Erbay, Burghout) no alcanzaron una exactitud tal que

\footnotetext{
27 Vidal, P. A., Ixtaina, P. R., “Application of a New Pavement Reflectometer”, 2nd Balkan Conference and Fair in Lighting Balkan-Light'02, Proceedings, Estambul, 2002; Ixtaina P., Vidal P., "Argentinean road surface characterization", International CIE Symposium on road surface photometric characteristics: Measurement Systems and Results, Torino, Italia, 2008; Ixtaina, P., Presso, "Estudio de Calzadas en las Autopistas Urbanas de la ciudad de Buenos Aires", Actas del X Congreso Panamericano de Iluminación, Valparaíso (Chile), Octubre de 2010, pp. 108-113.

${ }^{28}$ Vidal, P. A., Ixtaina, P. R., “New System of...”, op. cit. El autor de la presente Tesis se incorporó al grupo de trabajo del LAL como becario en 1992 y participó activamente en las experiencias que dieron lugar al trabajo expuesto en esta reunión de la CIE.

${ }^{29}$ CIE, Road surface and road marking..., 2001, op. cit.

${ }^{30}$ Burghout, F., "Two class system for...", op. cit.

${ }^{31}$ Según el desarrollo teórico planteado en: Vidal, P. A., Ixtaina, P. R., "New System of...”, op. cit.

${ }^{32}$ Range, H., "Road Reflectometer for...", op. cit.; Keitz, H. A. E, Light calculations and..., op. cit.
} 
permitiera mediciones confiables, por lo que son escasos los antecedentes de su utilización. Prueba de ello es que estos dispositivos prácticamente no avanzaron desde su faz de prototipos a modelos comerciales. 
Capítulo 4 - Medición de las propiedades reflectivas de las calzadas 
El presente capitulo aborda el tema central de la Tesis y tiene como eje los estudios realizados a pavimentos de instalaciones viales de la región en los últimos años. En la investigación pueden diferenciarse dos pasos o etapas:

a.- Medición de los parámetros del sistema de descripción. Este desarrollo original concluyó con el diseño y construcción de un equipo de medición (Reflectómetro de Calzadas) que puede trasladarse a la zona a evaluar en la autopista y que mide los parámetros de descripción con adecuada exactitud. La primera parte de este capítulo describe este sistema de medición, la base teórica que motivó su particular diseño y los métodos de calibración y prueba empleados. Se explica además el procedimiento aplicado para obtener finalmente una tabla-r a partir de los parámetros medidos sobre la calzada.

b.- Verificación experimental del equipo. La segunda parte del capítulo detalla las aplicaciones del equipo. Por un lado, describe un caso concreto de mejora en el diseño de la instalación: una instalación nueva, creada en base al pavimento estándar CIE R3 (como se dijo antes, esta era la práctica habitual en Argentina), cuya evaluación inicial de luminancias no fue coincidente con los datos del proyecto. Esta fue mejorada a partir de la caracterización de la calzada, re cálculo del sistema de alumbrado en base a este nuevo dato y una posterior modificación del enfoque de las luminarias. Similar tarea fue implementada en dos casos análogos de iluminaciones nuevas con la misma falla.

La segunda actividad, que surgió de los estudios anteriores, fue la transferencia tecnológica prestada a AUSA, a partir de la cual, se aplicó esta nueva tecnología en las autopistas bajo la concesión de esa empresa. Junto con lo anterior, este trabajo permitió lograr un registro global de las propiedades reflectivas de las calzadas en uso actualmente. ${ }^{1}$ El conjunto de tablas-r obtenidas fue posteriormente utilizado por concesionarios viales y empresas de iluminación para cálculos de obras nuevas, por ejemplo, la Autopista Acceso Oeste, el tramo cercano a Luján, la zona Ruta 5; la reconversión a LED de la Autopista 25 de mayo y Perito Moreno (AUSA) y La PataBuenos Aires. En todas estas obras, las evaluaciones de luminancia posteriores resultaron coincidentes (más allá de los lógicos errores de medición) con el proyecto.

Los estudios fueron expuestos en el simposio "Road Surface Photometric Characteristics: Measurements Systems and Results", convocado por la Comisión Internacional de Alumbrado CIE, División 4, en colaboración con INRIM (Istituto Nazionale di Ricerca Metrologica) y CERTU (Centre d'études sur les Réseaux, les Transports, l'Urbanisme et les constructions publiques). Este evento, realizado por fuera de las reuniones anuales de la CIE, fue organizado con la idea de generar un espacio para discutir la importancia de la caracterización fotométrica de las superficies de las carreteras. Allí se reunió a gran parte de los grupos de investigación que trabajan sobre el tema y las conversaciones evidenciaron la necesidad, a nivel mundial, de mejorar, extender y uniformar las prácticas relativas a la aplicación de la Técnica de Luminancia. ${ }^{2}$

Cabe destacar, finalmente, que las investigaciones fueron realizadas por el autor en el marco de su Plan de Trabajo como investigador de la Comisión de Investigaciones

\footnotetext{
${ }^{1}$ Las últimas aplicaciones del Reflectómetro de Calzadas datan de 2010/11. Pese a ello y de acuerdo con la información recabada de personal de las empresas concesionarias, se continúa empleando el mismo tipo de compuesto. La excepción serían las pavimentaciones detectadas en febrero de 2019 (ramal Tigre, Autopista Acceso Norte) que evidencian una tonalidad rojiza no presente en los ya tradicionales pavimentos del tipo drenante.

2 Rossi, G., "Synthesis of the discussions on "photometric measurements of Road surfaces", CIE symposium on road surface photometric characteristics - Measurement systems and results. Turin, 2008.
} 
Científicas (CIC), junto con el grupo Luminotecnia del Laboratorio de Acústica y Luminotecnia (LAL). Las posibilidades brindadas por el instrumento permitieron asimismo avanzar en otros aspectos más allá de la medición de las propiedades reflectivas, puntos que forman parte de la Tesis y se detallan en los capítulos 5, 6 y 7.

\section{1.- Medición de los parámetros del sistema de descripción}

La obtención de parámetros de descripción con instrumentos de campo se basa en la medición sobre la calzada de algunos coeficientes " $r$ ". Para ello, todos los equipos emiten luz hacia un sector de calzada (el área de medición) desde alguna dirección específica, que definirá luego el " $r$ " particular que se está midiendo. La luz reflejada por la calzada es medida por un detector, desde la posición de observación normalizada $\left(\alpha=1^{\circ}\right)$, rasante sobre el camino. ${ }^{3}$ Todos los instrumentos propuestos hasta la fecha para la medición de los parámetros del sistema de descripción (cualquiera sea este), se basaron en versiones portátiles compactas y debieron forzosamente ubicar al observador cercano a la porción de pavimento que se está evaluando.

Esto se observa tanto en los instrumentos desarrollados en la década del ' 80 (ver figuras 50 y 51, capítulo 3) como en las nuevas propuestas presentadas en el Simposio ya citado. De estas últimas, solo dos equipos fueron realmente implementados y probados "in situ", el desarrollado por Muzet, ${ }^{4}$ que se reproduce en la figura 53, y el de Laurent, ${ }^{5}$ en la figura 54.

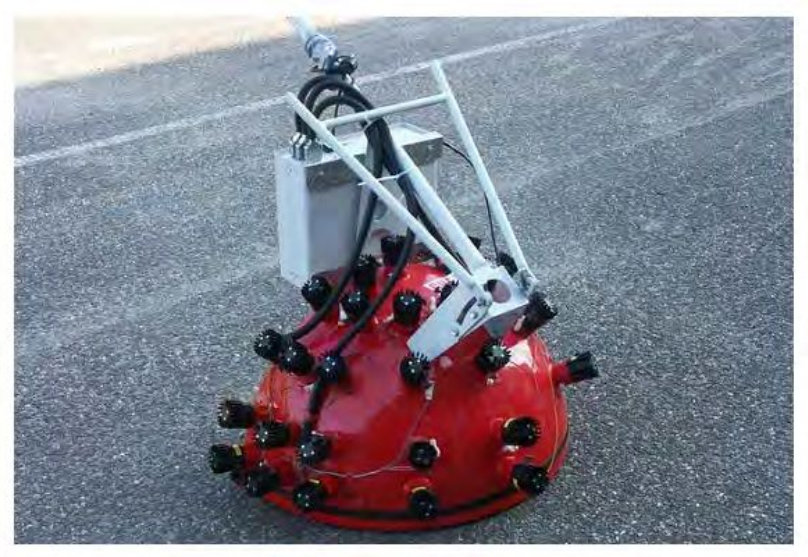

Figura 53. Prototipo de medidor compacto desarrollado por Muzet. ${ }^{6}$

\footnotetext{
${ }^{3}$ De Boer, J., Cohu, M., Schreuder, D., Public Lighting. Philips technical Library, Países Bajos, 1967.

${ }^{4}$ Muzet, V., "Coluroute: a mobile goniophotomer to characterize the road surface photometry", CIE International Symposium on road surface photometric characteristics: Measurement Systems and Results, Italia, 2008.

${ }^{5}$ Laurent, M., "Characterisation of Road Surfaces using Memphis mobile gonio reflectometer", CIE International Symposium on road surface photometric characteristics: Measurement Systems and Results, Italia, 2008.

${ }^{6}$ Imagen extraída de: Muzet, V., “Coluroute: a mobile...”, op. cit.
} 


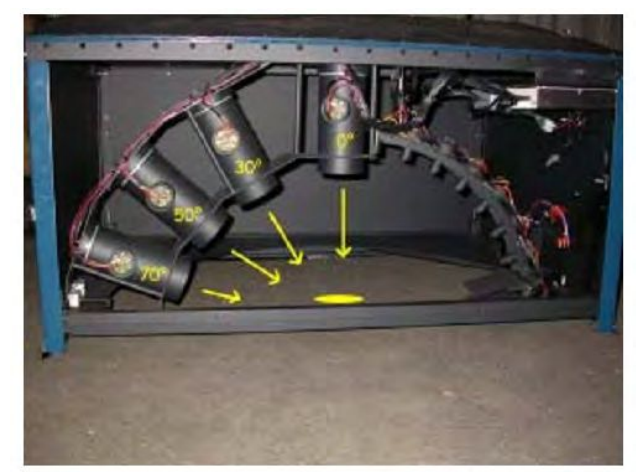

Figura 54. Prototipo de medidor compacto desarrollado por Laurent. ${ }^{7}$

Este último posee ángulos de observación mayores a $1^{\circ}$, entre $30^{\circ}$ y hasta $60^{\circ}$. Teniendo en cuenta lo ya dicho sobre la fuerte dependencia de las propiedades reflectivas con la posición de observación, el instrumento no arrojaría resultados compatibles con la aplicación estándar de la Técnica. De modo que el equipo citado ${ }^{4}$ sería la única propuesta acorde a la forma de medir la tabla-r estandarizada. Sobre este equipo y más allá de las pruebas citadas por Muzet respecto a la calibración del instrumento, no hay antecedentes de su uso concreto, al menos en la bibliografía consultada.

La idea central del desarrollo en esta Tesis es el enfoque a $1^{\circ}$, que resulta complejo si se pretende mantener una exactitud razonable. Refiriendo a la figura 55, un cálculo simple evidencia la dificultad de ajustar el ángulo $\alpha$, máxime si se contemplan las irregularidades propias de una calzada, que dista mucho de ser una superficie lisa.

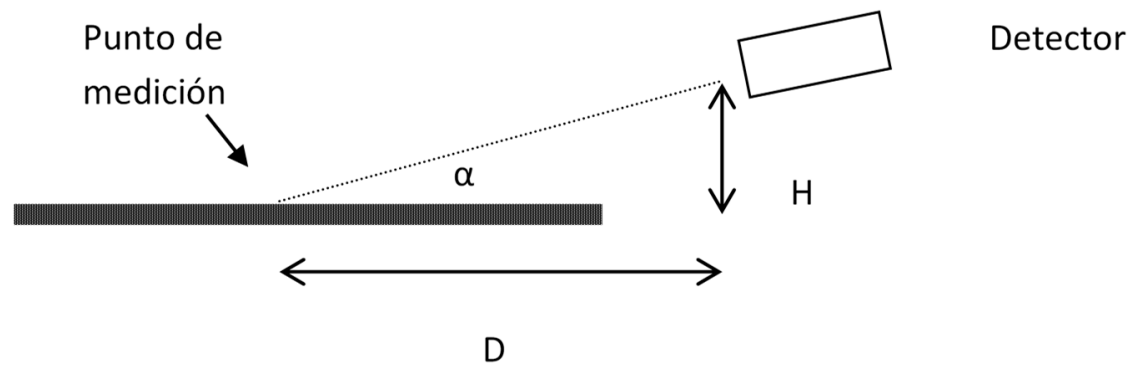

Figura 55. Esquema angular del enfoque (dibujo del autor).

Considerando:

$\mathrm{H}=\mathrm{D} . \operatorname{tg} \propto$ para $\mathrm{D} \approx 0.50 \mathrm{~m}, \mathrm{H}$ resulta $8,7 \mathrm{~mm}$

En términos aproximados, un equipo de $1 \mathrm{~m}$ de largo impone la ubicación del detector a menos de $10 \mathrm{~mm}$ de altura, lo que no es constructivamente sencillo. En los pavimentos del tipo drenante, las piedras superficiales sobresalen por lo general del nivel del aglutinante base entre 2 y $5 \mathrm{~mm}$. De este modo, considerando lo calculado en (1), y

\footnotetext{
${ }^{7}$ Imagen extraída de: Laurent, M., "Characterisation of Road...”, op. cit.
} 
suponiendo posible ajustar $\mathrm{H}$ a $8,7 \mathrm{~mm}$, las irregularidades introducirían un error al ubicarlo sobre la calzada que se traduciría en variaciones del ángulo $\alpha$ del orden $\pm 0,6^{\circ}$.

El instrumento implementado en las experiencias del autor usa un arco de circunferencia como guía de la fuente de luz, siguiendo la idea del prototipo de Range ${ }^{8}$ (ver capítulo 3 ). Sin embargo, se han modificado las escalas de modo de asegurar el ajuste de los ángulos de incidencia para las tolerancias inherentes al montaje del aparato sobre una superficie que no es perfectamente plana. Del mismo modo, la distancia entre el detector y la superficie de medición se incrementó considerablemente $(2,5 \mathrm{~m})$ lo que permitió asegurar con exactitud el ángulo de observación. La altura $\mathrm{H}$ de observación se incrementó así a $44 \mathrm{~mm}$, lo que permitió usar un detector comercial (luminancímetro) y generó una solución adicional, ya que, independizó al instrumento del tema central de la calibración.

La figura 56 muestra un esquema general del equipo. El prototipo construido consiste en dos sistemas independientes: la fuente luminosa y el detector. En el primero, se emplea un modelo de distancia fija a la superficie de medición; esta fuente describe un arco de circunferencia centrada en la zona del pavimento estudiada y soportada modo de riel, por el arco metálico.

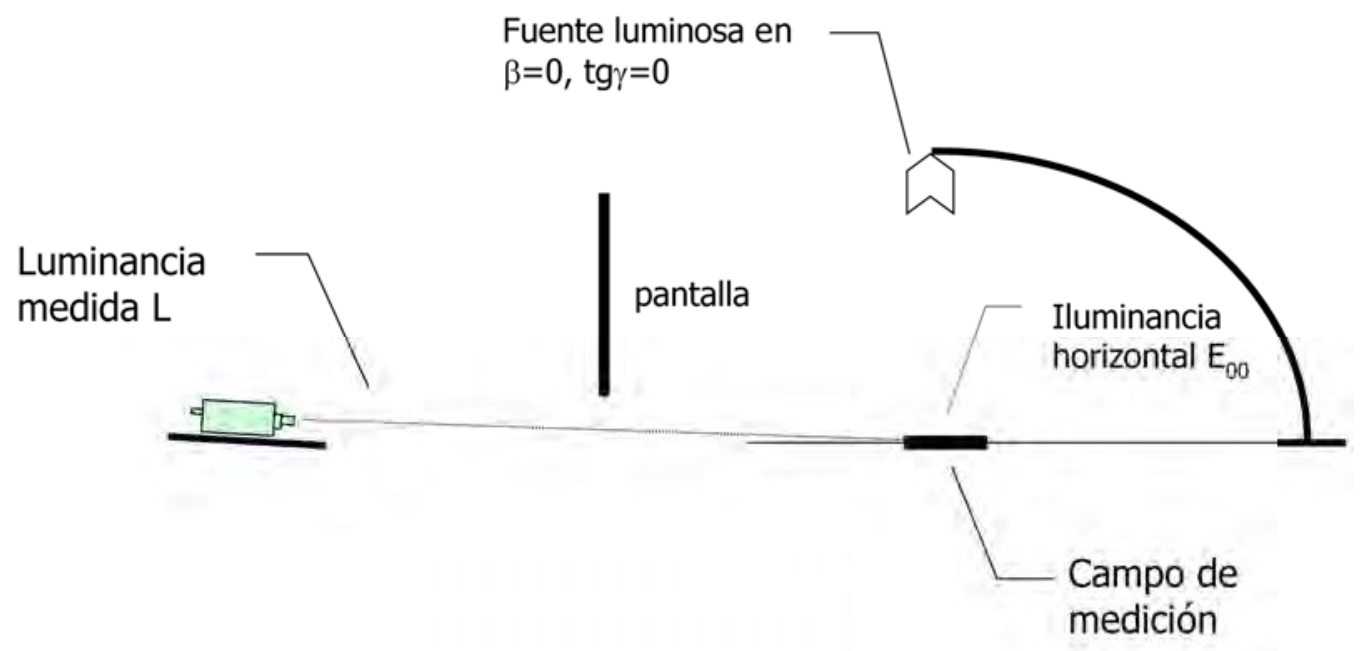

Figura 56. Esquema del Reflectómetro de Calzadas (dibujo del autor).

Como la idea es evaluar los valores "r" necesarios para el cálculo de los parámetros del sistema de descripción LAL-CIC (ver capítulo 3), ${ }^{9}$ la fuente debe poder barrer los planos $\beta=0^{\circ}$ y $\beta=90^{\circ}$, lo que se soluciona empleando un arco de un cuarto de circunferencia montado sobre un soporte, tal como indica la figura 57.

\footnotetext{
${ }^{8}$ Range, H., "Road Reflectometer for the simplified determination of the properties of lighted road surfaces", Lichttechnik, 25, p. 389, 1973.

${ }^{9}$ Vidal, P. A., Ixtaina, P. R., "New System of identification of de reflection indicatrices of pavements", note to CIE TC 4-25, Francia, 1996.
} 


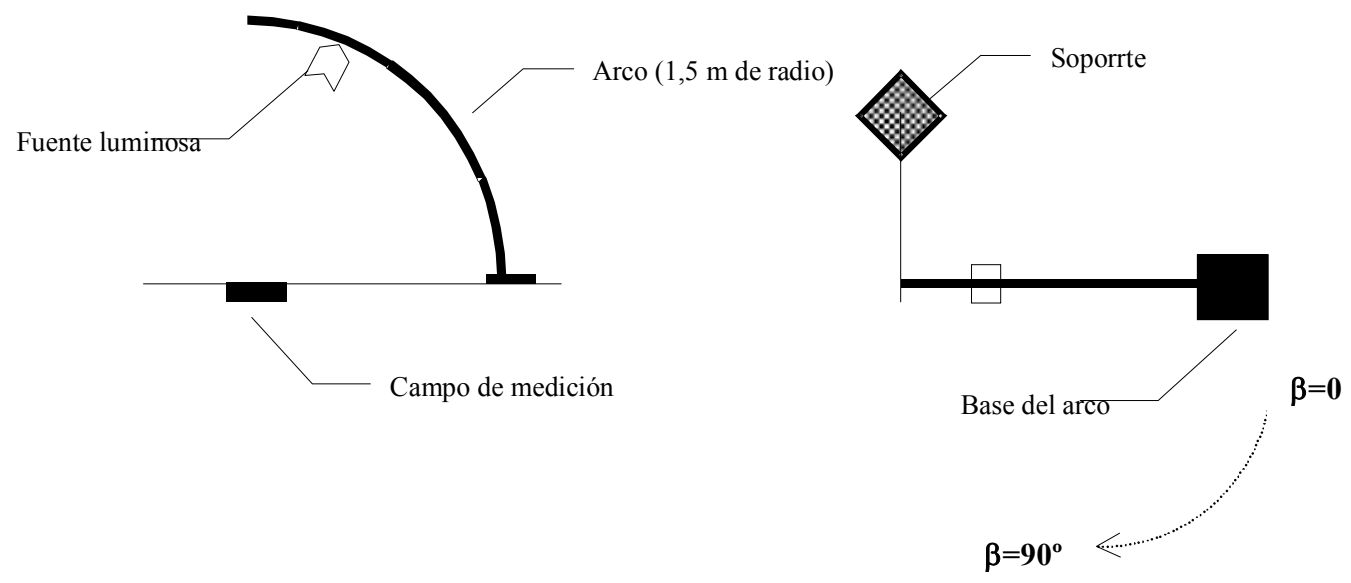

Figura 57. Esquema del sistema de iluminación de la zona a evaluar. ${ }^{10}$

Para solucionar los errores antes mencionados en la definición del ángulo de observación desde corta distancia, el sistema de detección empleó un luminancímetro comercial $\mathrm{LMT}^{11}$ ubicado sobre el piso a 2,50 $\mathrm{m}$ de distancia de la zona enfocada (área de medición), con un ángulo de enfoque de $1^{\circ}$. La apertura angular elegida para el luminancímetro permitió captar la luminancia de una porción de pavimento de aproximadamente $0,20 \mathrm{x}$ $0,40 \mathrm{~m}$, tamaño aproximado al normalizado para la medición de muestras de pavimento en laboratorio. ${ }^{12}$ El centro de la zona evaluada se hizo coincidir con la posición del nadir del montaje del arco (figura 58).

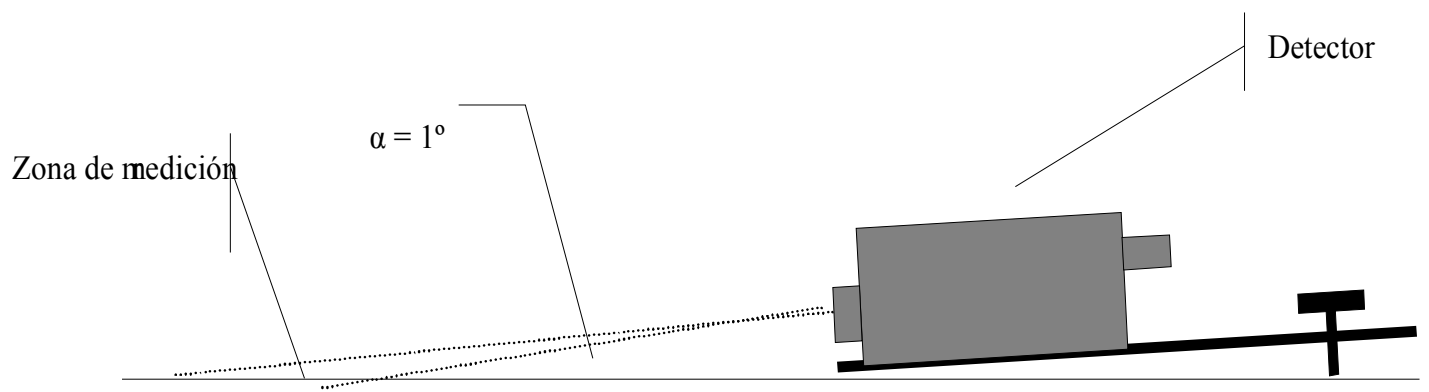

Figura 58. Montaje del detector. ${ }^{13}$

\footnotetext{
${ }^{10}$ Imagen extraída de: Ixtaina P., Vidal P., "Argentinean road surface characterization”, International CIE Symposium on road surface photometric characteristics: Measurement Systems and Results, Torino, Italia, 2008.

11 "Luminance Meter LMT 1009", LMT Lichtmesstechnik GMB, [en línea] URL: http://www.lmtberlin.de/en/11009.html [Consulta: 3 de abril de 2019].

${ }^{12}$ De Boer, J., Cohu, M., Schreuder, D., Public Lighting, op. cit.; CIE, Road surface and road marking reflection characteristics, publicación TC 4.24 y 4.25-144, Francia, 2001.

${ }^{13}$ Imagen extraída de: Ixtaina P., Vidal P., “Argentinean road surface...”, op. cit.
} 


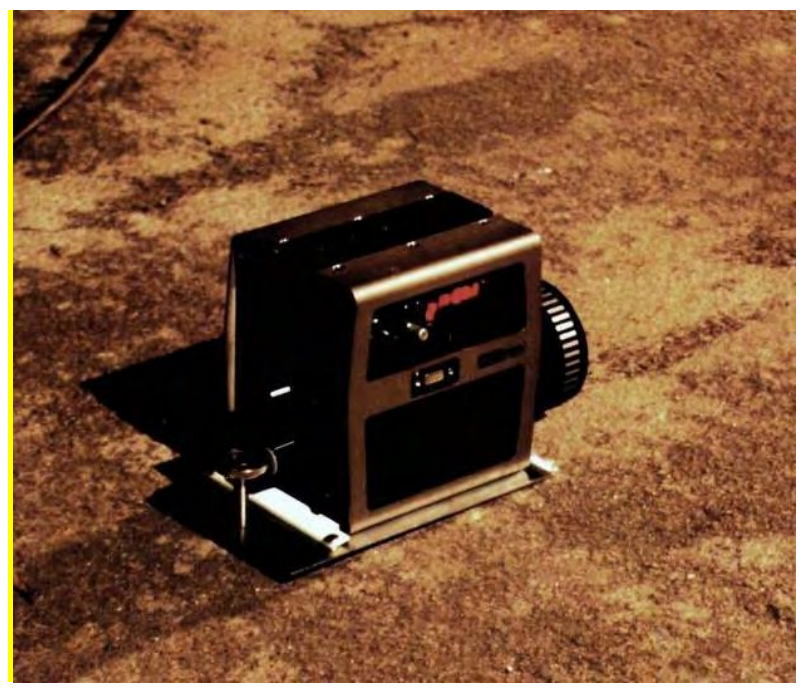

Figura 59. Aplicación del reflectómetro: detector de luminancia en posición de medición (foto del autor).

El luminancímetro se ubica sobre una pequeña placa metálica en cuya parte posterior se instala un tornillo fino. El mismo permite regular en forma estable el ángulo de observación y la zona enfocada.

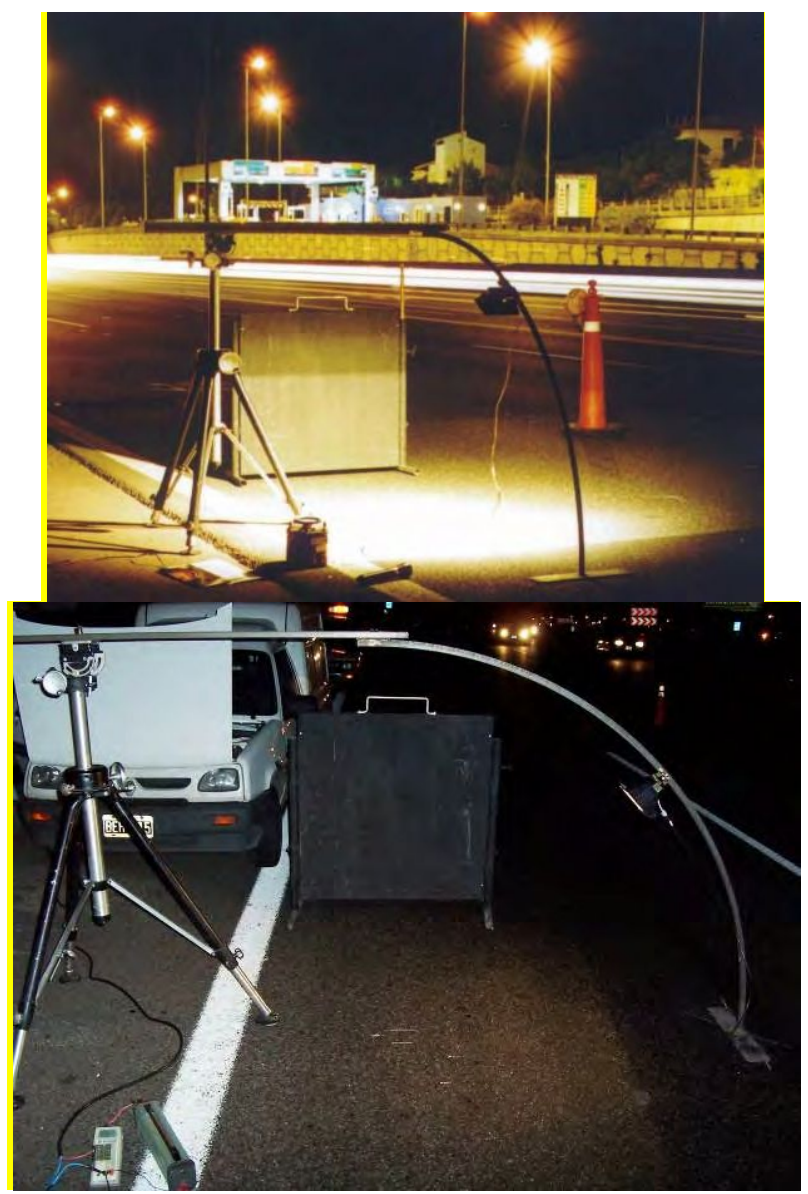

Figura 60. Aplicación del reflectómetro: sistema de iluminación (fotos del autor). 


\section{2.-Procedimiento de medición}

El arco soporte de la fuente luminosa se instala sobre una zona del camino convenientemente elegida para su caracterización. Debe ser representativa de la porción de ruta a evaluar, suficientemente plana y con pavimento homogéneo. El dispositivo de iluminación (arco) es nivelado para poder mantener constante la distancia entre la fuente y el centro del área de medición. Posteriormente, el detector es alineado con el arco, definiendo el plano $\beta=0^{\circ}-180^{\circ}$.

Con la fuente luminosa en la posición de zenit $(\beta=0, \operatorname{tg} \gamma=0)$ y utilizando pantallas, se verifica que el detector capture solo luz reflejada por el área bajo prueba (figura 61).

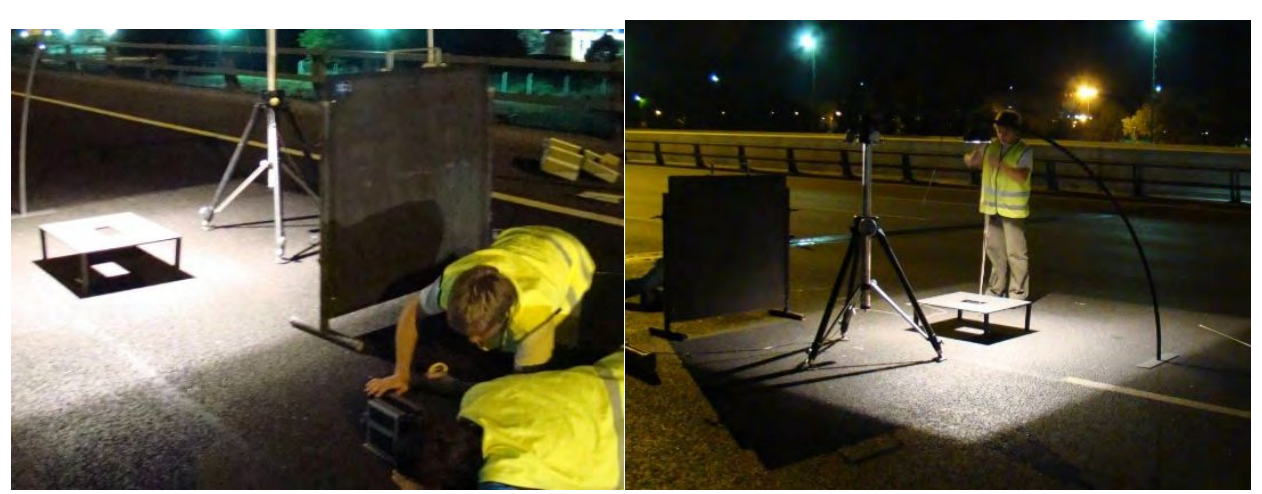

Figura 61. Aplicación del reflectómetro: alineación del detector (fotos del autor).

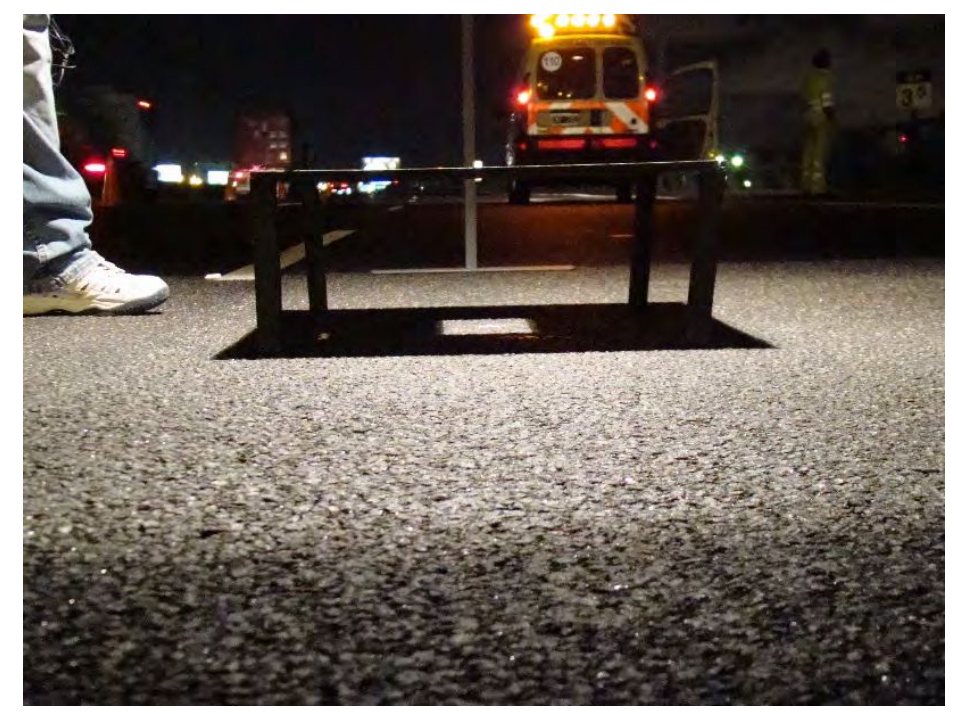

Figura 62. Ajuste del área de medición (fotos del autor).

En estas condiciones, el equipo es calibrado midiendo la iluminancia horizontal E 00 [lux], en el centro de la zona de prueba. La fuente luminosa (que se alimenta con tensión estabilizada para mantener constante su emisión) se ubica posteriormente en las posiciones $(\beta, \operatorname{tg} \gamma)$ definidas por los coeficientes del sistema de descripción ya mencionado y que repetimos: 


$$
\mathrm{A}_{\mathrm{D}}=\frac{\mathrm{r}_{(0,0.625)}}{\mathrm{r}_{(0,0)}} \quad \mathrm{A}_{\mathrm{I} 1}=\frac{\mathrm{r}_{(0,3)}}{\mathrm{r}_{(0,0.625)}} \quad \mathrm{A}_{\mathrm{I} 2}=\frac{\mathrm{r}_{(90,1)}}{\mathrm{r}_{(0,0.625)}}
$$

De este modo, los coeficientes de luminancia reducidos " $\mathrm{r}$ " se calculan a partir de la luminancia $\mathrm{L}_{(\beta, \mathrm{tg} \gamma)}[\mathrm{cd} / \mathrm{m} 2]$, medida por el detector para cada posición de la fuente de iluminación (ver capítulo 2):

$$
\mathrm{r}_{(\beta, \mathrm{tg} \gamma)}=\mathrm{L}_{(\beta, \mathrm{tg} \gamma)} / \mathrm{E}_{00} \cos ^{2} \gamma
$$

Por ejemplo, con el arco alineado $\left(\beta=0^{\circ}\right)$ y $\operatorname{tg} \gamma=3\left(\gamma=71,6^{\circ}\right)$ se mide la luminancia $\mathrm{L}_{(0,3)}$. Con ello se puede calcular el $\mathrm{r}_{(0,3)}$. El mismo procedimiento permite calcular el, por ejemplo, $\mathrm{r}_{(0,0,625)}$. Con ambos, es inmediato el cálculo de AI1. En total se necesitan cuatro posiciones de iluminación, lo que toma no más de 10 minutos de medición. Con ello se calculan posteriormente los parámetros de descripción $A_{D}, A_{I 1}, A_{I 2}$.

Antes de iniciar las pruebas de campo, el instrumento se ha ajustado y calibrado en el laboratorio, montándolo en una sala "oscura" del LAL, haciendo coincidir su centro con una muestra de pavimento de tabla-r conocida (muestra de referencia) (Figura 63). El procedimiento permitió verificar la exactitud en la determinación de los valores $r$ medidos. Asimismo, se repitió en varias oportunidades el armado y posterior medición, de modo de asegurar que las desviaciones propias del montaje queden enmarcadas en las incertidumbres admisibles para este tipo de mediciones. De este modo, se pudo verificar una incertidumbre expandida de $\pm 10 \%$ en la determinación de los coeficientes " $r$ ".
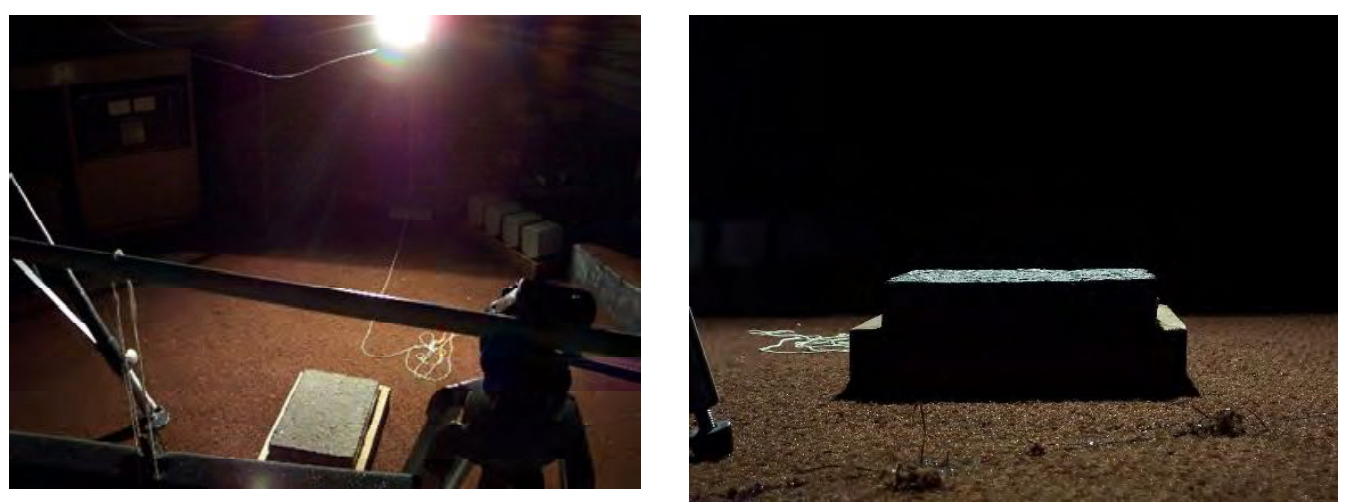

Figura 63. Calibración con muestras conocidas en el laboratorio (fotos del autor).

\section{3.- Obtención de la Tabla-r}

Para obtener una Tabla-r representativa del pavimento evaluado en la instalación y cuyos parámetros de descripción han sido medidos, se utilizó el procedimiento conocido como 
identificación. ${ }^{14}$ Este consiste en comparar los coeficientes de descripción medidos con los de las superficies estudiadas y almacenadas en bases de datos. Aquellas cuyos parámetros se aproximen a los medidos, tendrán características reflectivas similares, por lo que pueden utilizarse en los cálculos como representativas de la superficie real.

Se realizó la identificación, empleando las bases de datos de Erbay y Sorensen ya mencionadas en el capítulo $3 .{ }^{15}$ Estas compilan los resultados de más de 500 superficies medidas, agrupan distintos materiales y modalidades constructivas y contribuyen a identificar variadas formas de reflexión, que van de superficies muy difusas, a formas de alta especularidad. Si bien refieren a calzadas europeas, la cantidad y diversidad de tablas$r$ permite presuponer que dentro de la base sea altamente probable que exista alguna parecida a la del pavimento bajo estudio. Con el objeto de diversificar la fuente de comparación, la base de datos se completó con una veintena de pavimentos locales, medidos a partir de muestras extraídas de la provincia de Buenos Aires durante las décadas de los ' 80 y ' 90 .

\section{4.- Aplicaciones del Reflectómetro}

\section{1.- Pruebas iniciales sobre pavimentos nuevos o recapados}

\subsection{1.- Autopista Panamericana}

La autopista Panamericana, uno de los más importantes accesos a la Ciudad de Buenos Aires, fue escenario de la primera verificación del Reflectómetro. ${ }^{16}$ La prueba se basó en la caracterización de tres zonas "testigos" con distinto tipo de pavimento. Se empleó para ello el instrumento experimental, que permitió medir directamente sobre la calzada los parámetros $A_{D}, A_{I 1}$ y $A_{I 2}$. Posteriormente y mediante identificación, se asignaron tres tablas-r como aptas para representarlos. Al mismo tiempo, se extrajeron muestras de cada zona "testigo", efectuando cortes en el pavimento en exactamente el mismo lugar dónde se midieron los parámetros. Los materiales fueron llevados al laboratorio y allí se midieron en forma completa, empleando el método tradicional (reflectómetro de muestras).

La diferencia entre las tablas-r obtenidas por identificación y las medidas en forma completa fueron cuantificadas usando el método descrito en un trabajo propio ya citado, ${ }^{17}$ que se basa en la comparación directa de tablas-r según el siguiente razonamiento:

Se calculan los cocientes $Z_{(\beta, \text { tan } \gamma)}$ para todos los valores que componen ambas tablas-r (tabla-r $r_{i}$ y tabla- $\left.r_{j}\right)$.

\footnotetext{
${ }^{14}$ De Boer, J., Van Bommel, W., Road Lighting, Philips technical Library, Países Bajos, 1980; Burghout, F., "Two class system for the classification of dry road surfaces with regard to light reflection", CIE Journal 5(1), pp. 22-43, 1986.

${ }^{15}$ Erbay, A., Atlas of the reflection properties of road surfaces, ILTUB, Berlin, 1974a; Vidal, P. A., Ixtaina, P. R., "New method for characterising the reflection properties of road surfaces", CIE 25 th Session, San Diego, 2003.

${ }^{16}$ Vidal, P. A., Ixtaina, P. R., "New method for characterising...", op. cit.; Vidal, P. A., Ixtaina, P. R., "Application of a New Pavement Reflectometer", 2nd Balkan Conference and Fair in Lighting BalkanLight'02, Proceedings, Estambul, 2002.

${ }^{17}$ Vidal, P. A., Ixtaina, P. R., "New System of...”, op. cit.
} 


$$
Z(\beta, \tan \gamma)_{i j}=\frac{r(\beta, \tan \gamma)_{i}}{r(\beta, \tan \gamma)_{j}}
$$

Si ambas tablas-r tuvieran la misma forma pero distinto grado de "claridad", los coeficientes $r$ en la misma posición en ambas diferirían tan solo en un factor de escala, de modo que los cocientes $Z$ serían constantes (razonamiento ya empleado por Burghout. ${ }^{18}$ ). Dado que éste no es el caso que se presenta ( $Z$ no es constante), se calculó el valor medio de $Z$ y se empleó su desviación estándar para evaluar la diferencia entre ambas tablas-r.

Los resultados de este procedimiento, para cada una de las tres superficies estudiadas, se muestran en la tabla 3 .

\begin{tabular}{|c|c|c|c|c|}
\hline Superficie & $\begin{array}{c}\text { parámetros de } \\
\text { descripción } \\
\text { medidos "in situ" }\end{array}$ & $\begin{array}{c}\text { Identificada } \\
\text { como: }\end{array}$ & $\begin{array}{c}\text { Parámetros de } \\
\text { descripción } \\
\text { medidos en } \\
\text { muestras }\end{array}$ & $\begin{array}{c}\text { Diferencias } \\
\sigma_{\mathrm{n}-1} /\left(\mathrm{Z}_{\mathrm{ij}}\right) \text { av } \\
{[\%]}\end{array}$
\end{tabular}

Tabla 3. Sumario de la comparación entre tablas-r. ${ }^{19}$

La dispersión calculada para los cocientes $Z$, que promedia el $\pm 30 \%$, es consistente con las incertidumbres de medición, considerando que ambos métodos (medición con reflectómetro y sobre muestras) son independientes.

\subsection{2.- Autopista del Oeste}

En este segundo caso, se encontró la siguiente situación: transcurridos dos años de su inauguración (junio de 1998), y en pleno desarrollo de obras de mantenimiento, se reemplazó el pavimento en un tramo de la ruta, cambiando un asfalto tradicional por otro del tipo "drenante". La modificación alteró la calidad del sistema de iluminación, observándose en esta zona una drástica disminución de la uniformidad longitudinal Ul, que cayó de 0,7 a valores cercanos a 0,5 (0,7 es el valor límite mínimo según la norma IRAM AADL J 2022-2, ver capítulo 2).

A modo de solución, se implementó el siguiente plan de trabajo:

\footnotetext{
${ }^{18}$ Burghout, F., “Two class system...”, op. cit.

${ }^{19}$ Tabla extraída de: Vidal, P. A., Ixtaina, P. R., "New method for...”, op. cit.
} 
a.- medición de los parámetros de descripción del nuevo sistema con el nuevo equipo;

b.- obtención, vía identificación, de una tabla-r apta para representarlo;

c.- estudio de la posibilidad de adaptar (reenfocar) las luminarias de acuerdo con la matriz de reflectancia obtenida en el punto anterior, a fin de mejorar la uniformidad en la iluminación.

La matriz identificada (cuya tabla-r, dibujada en un diagrama espacial, se muestra en la figura 64) resultó ser una superficie de "alta especularidad", con un "pico" muy pronunciado. Como se comprende fácilmente, tal diagrama implica zonas en las que hay una importante reflexión de luz hacia el observador ("picos"), mientras que refleja muy poco para otros ángulos de incidencia. Se concluye entonces que esta superficie es completamente diferente al estándar R3 usado en el diseño inicial de la instalación (cuyo diagrama se incluye en la figura).
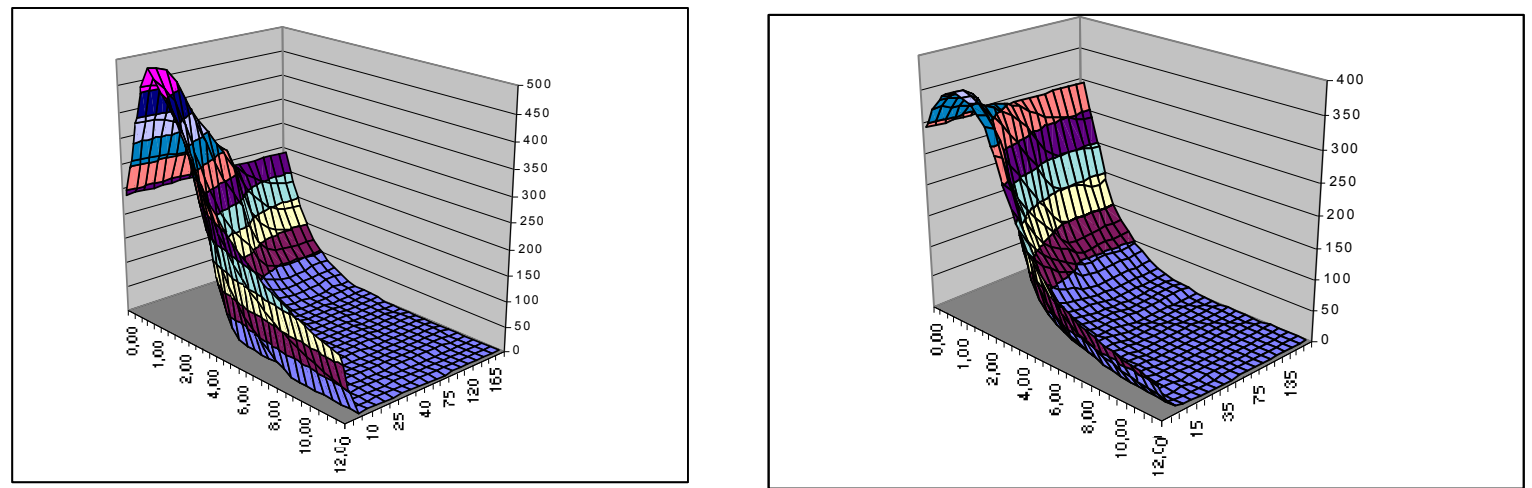

Figura 64. Diagrama espacial de la superficie estudiada (izquierda) y del estándar R3 (derecha). ${ }^{20}$

El paso siguiente fue enfocar las luminarias, de modo de dirigir la luz de acuerdo a las imposiciones de la matriz de reflexión hallada. Ésta tarea la realizó el fabricante de las luminarias, quien posteriormente y a modo de prueba final, reemplazó en la autopista un grupo de luminarias originales por las re-enfocadas. En esta zona tomada como testigo, se efectuó un relevamiento convencional de luminancias puntuales, cuyos resultados resumidos (junto con las mediciones originales) se presentan en la tabla 4.

\begin{tabular}{|c|c|c|c|c|c|c|c|c|c|c|c|c|c|c|}
\hline Test & \multicolumn{13}{|c|}{ Luminancias puntuales en la línea central del carril rápido $\left[\mathbf{c d} / \mathbf{m}^{2}\right]$} & UI \\
\hline $\begin{array}{c}\text { Medición original ('98 } \\
\text { - antes de los trabajos } \\
\text { de repavimentación) }\end{array}$ & 6,80 & 6,80 & 7,60 & 7,80 & 8,40 & 9,40 & 9,60 & 9,70 & 9,70 & 9,60 & 8,80 & 8,00 & 7,50 & 0,70 \\
\hline $\begin{array}{l}\text { Estado Actual } \\
\text { (después de la } \\
\text { repavimentación) }\end{array}$ & 4,30 & 4,50 & 5,10 & 6,30 & 7,60 & 8,70 & 8,60 & 8,40 & 8,10 & 7,10 & 6,40 & 5,60 & 4,90 & 0,49 \\
\hline $\begin{array}{l}\text { Mediciones sobre la } \\
\text { zona de prueba } \\
\text { (después de reenfocar } \\
\text { las luminarias) }\end{array}$ & 3,50 & 3,80 & 4,10 & 4,30 & 4,80 & 5,10 & 5,10 & 4,80 & 4,60 & 4,40 & 4,30 & 4,10 & 3,90 & 0,69 \\
\hline
\end{tabular}

Tabla 4. Luminancias puntuales en un vano típico, tomado como testigo. ${ }^{21}$

\footnotetext{
${ }^{20}$ Diagrama extraído de: Vidal, P. A., Ixtaina, P. R., “Application of a New...”, op. cit.

${ }^{21}$ Ibídem.
} 
La tabla-r obtenida mediante el proceso de descripción (con el reflectómetro) e identificación, constituye una mejora importante en la aproximación al pavimento real de la instalación. Por esta vía se evita la extracción y posterior medición de muestras, procedimiento útil para fines de investigación, pero difícil de implementar en el caso de obras concretas. ${ }^{22}$

\section{2.- Pruebas sobre pavimentos en uso}

Se describe aquí un estudio de las propiedades reflectivas de los pavimentos de las autopistas que integran la Red de Autopistas Urbanas de la Ciudad de Buenos Aires, realizado en el período 2008/2009. La actividad constituyó una transferencia tecnológica solicitada por la empresa AUSA que consideró necesario contar con tablas-r reales de las calzadas de su red, en vistas al futuro recambio del sistema de iluminación a LED. Se analizaron zonas típicas, cubriendo la totalidad de los ramales de la red y abarcando compuestos asfálticos tradicionales y macro texturados. Los resultados de esta investigación fueron presentados en el X Congreso Panamericano de Iluminación, Valparaíso (Chile), en octubre de $2010 .^{23}$

\subsection{1.- Autopista AU6 - "Perito Moreno"}

Esta medición trabajó sobre un pavimento con alta rugosidad, color oscuro (aspecto tipo calzada húmeda), con un ligero cambio de textura (menor rugosidad) desde aproximadamente la mitad derecha del ancho total de la calzada. Esta modificación era muy notoria en casi la totalidad del carril lento y de la banquina. Se efectuaron cuatro grupos de mediciones con el reflectómetro, verificándose una aceptable dispersión en los parámetros de descripción obtenidos en la zona central de la calzada. La dispersión fue mayor en la zona de banquina, lo que probablemente indicaba una falta de homogeneidad en esta porción de calzada. Como resultado, se obtuvieron dos tablas-r, cuyos diagramas espaciales se muestran en las figuras 65 y 66.

\footnotetext{
${ }^{22}$ Los detalles de las investigaciones cuya síntesis se muestra en los puntos 4.1.1 y 4.1.2 se encuentran en: Vidal, P. A., Ixtaina, P. R., “Application of a New...”, op. cit.; Vidal, P. A., Ixtaina, P. R., "New method for...", op. Cit.

${ }^{23}$ Ixtaina, P., Presso, "Estudio de Calzadas en las Autopistas Urbanas de la ciudad de Buenos Aires", Actas del X Congreso Panamericano de Iluminación, Valparaíso (Chile), Octubre de 2010, pp. 108-113.
} 


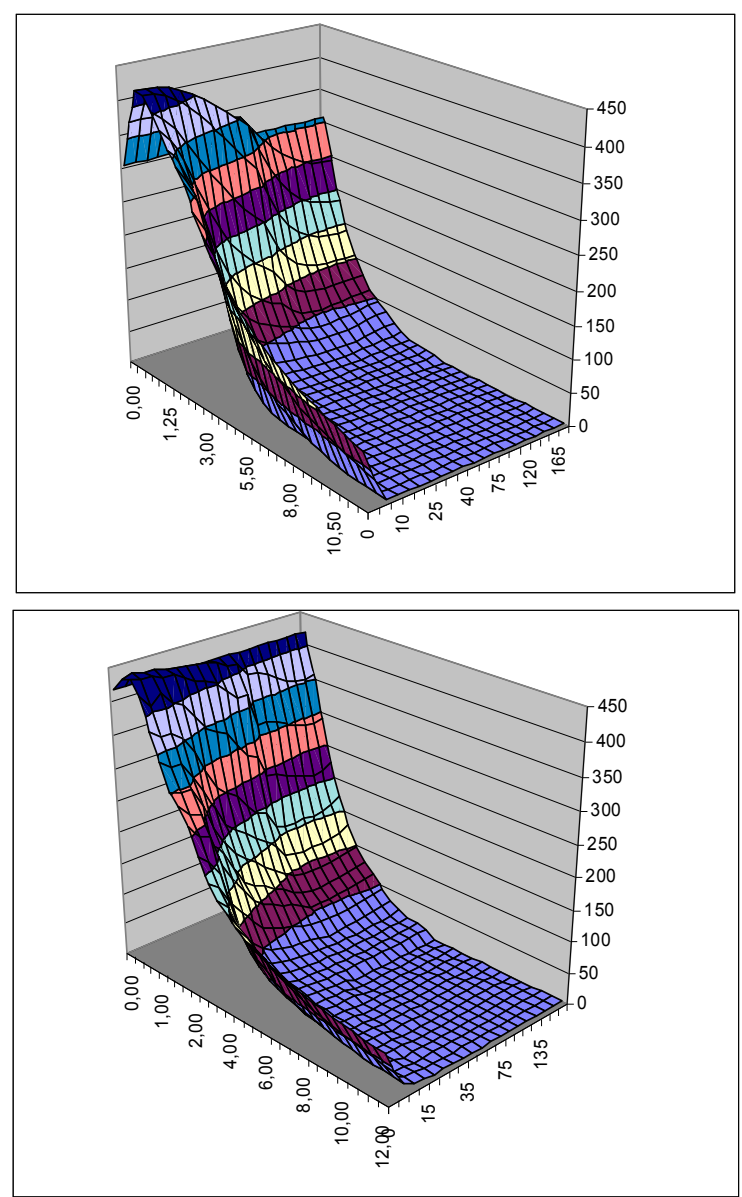

Figura 65. Autopista AU6. Zona central de la calzada. Figura 66. Autopista AU6. Carril lento y banquina. $^{24}$

\subsection{2.- Autopista "9 de Julio Sur"}

La inspección visual previa de esta autopista indicaba una superficie de alta rugosidad y oscura sin evidencias de cambio de color o textura. Al igual que en el caso anterior, la calzada poseía una elevada carga de tránsito, pudiendo en este caso constatarse más de siete años de uso sin alteraciones (recapados) de importancia. Como dato adicional de interés, el tipo de compuesto asfáltico había sido aplicado en forma casi simultánea en otra autopista de la red (autopista 25 de Mayo) cuyas secciones se habían caracterizado en un estudio previo (años 2005/2006) con resultados coincidentes. La figura 67 muestra el diagrama espacial de la tabla -r obtenida.

\footnotetext{
${ }^{24}$ Ibídem.
} 


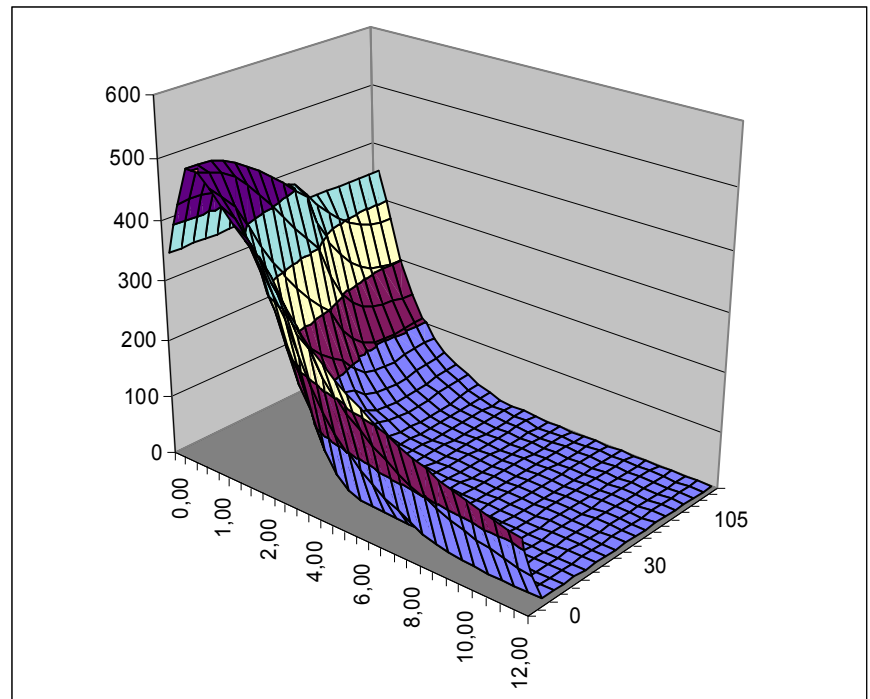

Figura 67. Tabla-r obtenida para representar las calzadas en Autopista 9 de Julio Sur. ${ }^{25}$

\subsection{3.- Autopista "Arturo Illia”}

En este caso, la inspección visual previa no resultó en un cambio de coloración o textura. Sin embargo, la dispersión en los parámetros de descripción obtenidos determinó la creación de dos tablas-r para representar la calzada de esta vía de tránsito. Aunque las matrices sean muy similares entre sí, una de ellas sería la más aconsejable para la zona central de la calzada (figura 68) mientras que la segunda (figura 69) sería la que más se aproxima al pavimento del carril exterior.

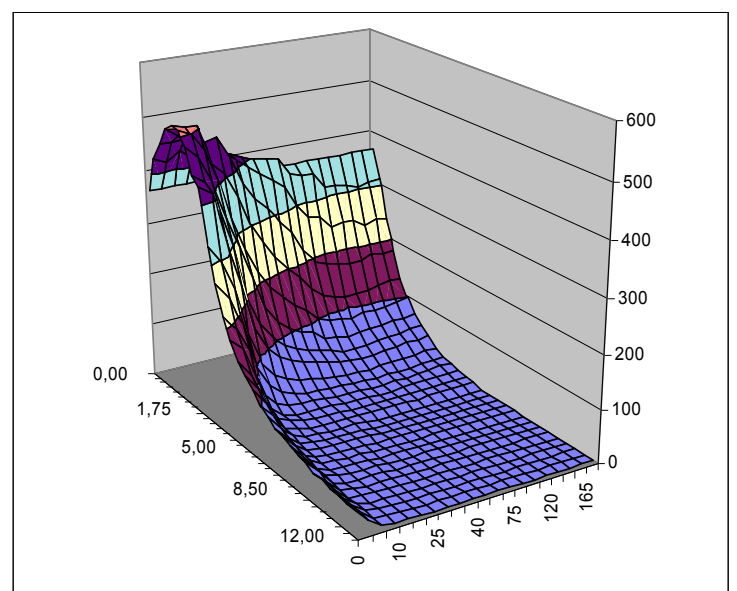

Figura 68. Autopista Ilia.

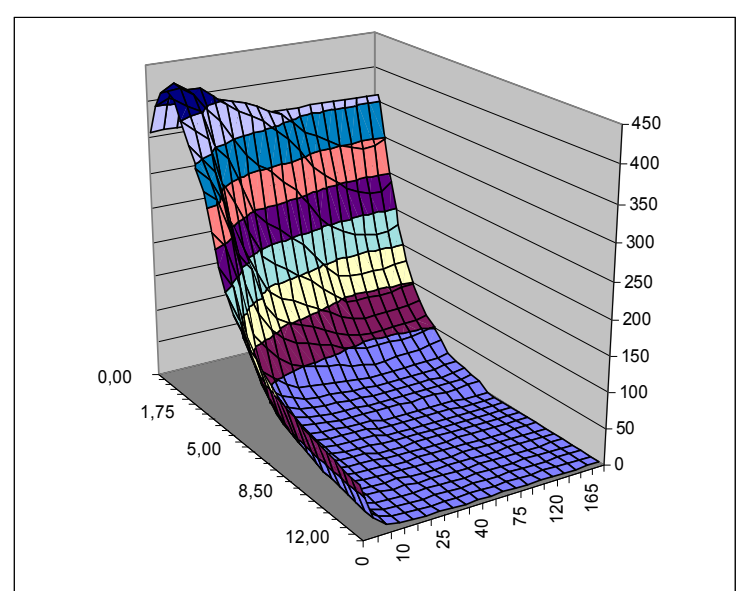

Figura 69. Autopista Ilia. ${ }^{26}$

Las formas de reflexión encontradas se corresponden con pavimentos de elevada especularidad con "picos" claramente definidos. No entrarían en este análisis las tablas correspondientes a carriles externos, aunque estos pavimentos distan de corresponderse con superficies difusas. Pese a que los índices de especularidad S1 (sistema CIE) se

\footnotetext{
${ }^{25}$ Ibídem.

${ }^{26}$ Ibídem.
} 
aproximan a los del estándar R3 $(\mathrm{S} 1=1,11)$ las tablas-r no evidencian una coincidencia, hecho que surge de compararlas con el diagrama espacial de dicho estándar. Este dato no es menor puesto que la superficie R3 era la típicamente usada por diseñadores y proyectistas en los diseños locales de sistemas de alumbrado.

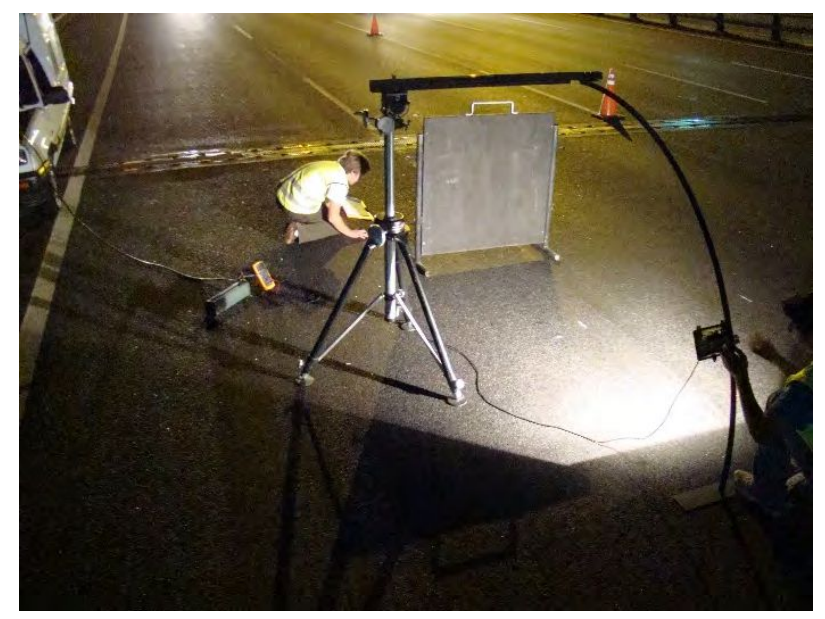

Figura 70. Caracterización de calzadas en la autopista Illia (Fotos del autor)

\section{3.- Caracterización de pavimentos locales}

A modo de síntesis de las evaluaciones efectuadas en el período 2001-2009, se presentan en la tabla 3 las características más relevantes de las superficies en uso en las principales autopistas argentinas. Se muestran los parámetros del sistema CIE y LAL-CIC, junto con el "perfil" de valores $r$, que resulta útil para una rápida comparación cualitativa. Cada juego de datos se corresponde con la tipificación de la calzada en un cierto tramo o sector. Los parámetros indicados son los considerados característicos y surgen de varias mediciones efectuadas con el reflectómetro en áreas aledañas. Por ejemplo, es natural encontrar diferencias (notorias incluso a simple vista) entre el centro del carril y sus bordes (huella), las cuales se repiten con diferentes matices en el sentido transversal de la vía de tránsito. Con ello, cada pavimento evaluado se traduce en un juego de parámetros de descripción y la matriz identificada "típica" se obtiene a partir de los parámetros medios.

\begin{tabular}{|c|c|c|c|c|c|c|c|}
\hline \multirow{2}{*}{ Pavimento } & \multirow{2}{*}{ Año } & \multicolumn{2}{|c|}{ Parámetros CIE } & \multicolumn{3}{|c|}{ Parámetros LAL } & \multirow{2}{*}{ Perfil } \\
\hline & & s1 & Qo & AD & Al1 & Al2 & \\
\hline HS1 & 2001 & 1,65 & 0,062 & 1,549 & 0,734 & 0,219 & Figura A1 \\
\hline HS2 & 2001 & 1,74 & 0,063 & 1,770 & 0,817 & 0,202 & Figura A2 \\
\hline HS3 & 2002 & 2,66 & 0,083 & 2,007 & 1,100 & 0,148 & Figura $A 3$ \\
\hline HS4 & 2002 & 1,47 & 0,095 & 1,666 & 0,286 & 0,359 & Figura A4 \\
\hline MS1 & 2003 & 0,44 & 0,076 & 1,096 & 0,235 & 0,414 & Figura A5 \\
\hline MS2 & 2005 & 1,14 & 0,074 & 1,422 & 0,669 & 0,228 & Figura A6 \\
\hline HS5 & 2005 & 1,73 & 0,084 & 1,599 & 0,805 & 0,217 & Figura A7 \\
\hline
\end{tabular}


Tabla 5. Características de reflexión de las superficies estudiadas. ${ }^{27}$

La denominación técnica de las superficies, junto con los datos relevantes desde el punto de vista macroscópico (aspecto, grado cualitativo de claridad y tiempo de rodamiento), se indican en la tabla 6.

\begin{tabular}{|c|c|c|c|}
\hline Pavimento & Denominación & $\begin{array}{l}\text { Tiempo de } \\
\text { rodamiento }\end{array}$ & Aspecto cualitativo \\
\hline HS1 & \multirow{4}{*}{$\begin{array}{c}\text { Micros concretos asfálticos discontinuos en } \\
\text { caliente, de elevada macro textura y con asfaltos } \\
\text { modificados }\end{array}$} & 5 meses & \multirow{2}{*}{$\begin{array}{l}\text { Elevada macro textura, piedras grises } \\
\text { visibles en superficie, muy oscuro } \\
\text { (aspecto mojado). }\end{array}$} \\
\hline HS2 & & Aprox. 6 meses & \\
\hline HS3 & & 1 año & \multirow{2}{*}{$\begin{array}{l}\text { Textura media, color gris claro, } 50 \% \\
\text { piedras visibles en superficie. }\end{array}$} \\
\hline HS4 & & 1 año & \\
\hline MS1 & $\begin{array}{l}\text { Concreto asfáltico en caliente, con asfaltos } \\
\text { modificados }\end{array}$ & 8 meses & $\begin{array}{c}\text { Baja macro textura, } 20 \% \text { piedras visibles } \\
\text { en superficie, oscuro }\end{array}$ \\
\hline MS2 & \multirow{2}{*}{ Micros concretos asfálticos discontinuos en caliente } & 1 1 1/2 año & \multirow{2}{*}{$\begin{array}{l}\text { Elevada macro textura, piedras grises } \\
\text { visibles en superficie, oscuro. }\end{array}$} \\
\hline HS5 & & $1 \frac{1}{2}$ año & \\
\hline
\end{tabular}

Tabla 6. Denominación técnica de superficies y aspecto. ${ }^{28}$

Es importante destacar que, prácticamente la totalidad de las superficies caracterizadas fueron empleadas en el re-diseño del sistema de alumbrado o bien directamente en el proyecto de la instalación. En todos los casos, los resultados finales fueron satisfactorios, lo que permite presuponer una cierta concordancia entre la tabla-r y el pavimento real de la instalación. La afirmación se deriva de una verificación práctica del método de trabajo basado en la caracterización "in situ" de la superficie y una sustancial mejora con respecto a la aplicación local de los estándares CIE.

Por otra parte, y atendiendo a las propiedades de las superficies caracterizadas, puede observarse un claro predominio de pavimentos altamente especulares, hecho que ya se había manifestado en las primeras aplicaciones del reflectómetro. De acuerdo a las mediciones realizadas, todo parece indicar que los compuestos "drenantes", de elevada macro textura, poseen un notorio "pico" de reflexión, independientemente de su "color" o "brillo" observado a simple vista (notar la descripción del tipo HS1/HS2 con respecto al HS3/HS4). Debe remarcarse que estos compuestos están siendo usados intensivamente, hecho que no debería descuidarse al momento del diseño de una nueva instalación, ya que evidentemente tales tablas-r dificultan notoriamente la obtención de buenos niveles de regularidad y exigen luminarias con cuidados diagramas de emisión.

Finalmente, lo expuesto en el presente punto constituye la primera caracterización de calzadas a nivel zonal. Las tablas-r encontradas en la evaluación descripta equivaldrían al sistema CIE R1 mencionado, pero adecuado espacialmente a las zonas aledañas a la ciudad de Buenos Aires y temporalmente a los pavimentos en uso en esta región en los últimos 10 años aproximadamente. El Anexo I muestra en forma gráfica las características reflectivas de las tablas-r de este sistema local de clasificación de calzadas.

\footnotetext{
${ }^{27}$ Ibídem.

${ }^{28}$ Ibídem.
} 
Capítulo 5 - Evolución de las propiedades reflectivas de las calzadas 
Luego de haber presentado lo esencial de la fisiología visión y los lineamientos generales de la luminotecnia (capítulo 1), las particularidades del alumbrado vial (capítulo 2) y la técnica de luminancia, su diseño e implementación (capítulo 3), se evidenciaron en el capítulo 4 las cuestiones no resueltas que justifican las hipótesis de la Tesis. A la luz de los resultados de las aplicaciones más recientes de la técnica de luminancia en Argentina, se verificó la hipótesis que remarca que los pavimentos locales son muy distintos de los estándares europeos, por lo que no debería usarse la serie $\mathrm{R}$ en los diseños de iluminación para la región. El capítulo finalizó exponiendo los detalles de un instrumento y método novedoso para obtener la tabla-r a partir de mediciones en el lugar, técnica que pudo ser validada con varias aplicaciones en autopistas de la región.

Los datos recabados en las experiencias anteriores posibilitaron dar cuenta de otros tres aspectos de la Técnica de Luminancia:

1. La tabla-r no es un dato "estático", por el contrario, presenta variaciones propias del uso y envejecimiento. Se afirma, de acuerdo a lo postulado en la introducción, que la evolución de los pavimentos locales no se da de la misma manera que lo informado en estudios (escasos) realizados en el extranjero sobre el tema. Esta constatación permite concretar un argumento importante: la modificación a la que se refiere influye mucho en la variación de los parámetros lumínicos durante la vida de la instalación y resulta necesario particularizarlos lo mejor posible, teniendo en cuenta los contextos de fabricación y aplicación.

2. En segundo lugar, el estudio de las particularidades de los pavimentos locales (sin equipararlos a los europeos) resulta clave, dado que el tipo reflexión que provoca la calzada tiene un importante efecto sobre la eficiencia energética de la instalación. Es así que la elección adecuada de la calzada incidirá directamente en el consumo de energía.

3. Por último, y luego del recorrido planteado en el estudio de los pavimentos, se plantea una tercera cuestión vinculada a la iluminación: la reflexión en la calzada depende del espectro de la fuente, y esta dependencia posee particularidades para la iluminación LED. Si bien existen antecedentes de dependencia de la tabla-r con el espectro, los estudios hallados en la bibliografía no hacen referencia a esta nueva forma de iluminar.

El presente apartado se focaliza el primer punto. Los otros dos serán trabajados en los capítulos 6 y 7.

\section{1.- La importancia del estudio de los cambios en las propiedades reflectivas}

En los últimos años, los análisis acerca del alumbrado público en calles y autopistas se han focalizado más en el tipo de luminaria utilizada, que en las cualidades reflectivas de las calzadas.

La irrupción de los leds en el alumbrado vial ha modificado las pautas tradicionales de diseño, configurando un cambio tecnológico que propone una instalación con mayor costo inicial y menor consumo de energía. Esto quiere decir que, por un lado, 
la relación de precios entre luminaria led y luminaria tradicional es cercana a 3 a $1^{1}$ pero, por otra parte, que la mayor eficiencia energética del primer tipo permite mantener adecuados niveles de iluminación con una menor potencia instalada. La tendencia es sin embargo, a equiparar costos en un futuro cercano lo que sin duda conllevará a una notable disminución del mercado de las lámparas de descarga e incluso su desaparición (al menos en el alumbrado público).

Es así que la clave del diseño actual de una instalación de alumbrado vial se concentra en cuestiones vinculadas al mencionado cambio tecnológico, como por ejemplo, la clase de led a emplear, la lente adecuada para las características de la calzada, la relación de eficacia con respecto a la fuente tradicional (sodio alta presión), la temperatura de color de la luz y el espectro, entre otras. En este sentido, el "impacto" (incluso desde un punto de vista comercial) asociado a la conversión a led relega y resta importancia a aspectos centrales de los diseños viales, como son el efecto de las propiedades reflectivas de las calzadas y la depreciación de la instalación.

El desgaste producido por el tránsito, así como las condiciones ambientales (radiación solar, lluvia o suciedad) modifican paulatinamente las características reflectivas (en otras palabras, la matriz de reflexión, su tabla-r) de los pavimentos. En una instalación de alumbrado vial, este cambio altera la distribución de luminancias sobre la calzada y con ello las condiciones de percepción. Se afirma, por consiguiente, que a pesar de la mejora que genera el uso de leds, este fenómeno, conocido como depreciación, afecta a los sistemas de alumbrado en general y también a las instalaciones viales diseñadas a partir de la técnica de luminancia.

Para constatar estas afirmaciones, se analizan aquí los cambios que produce el uso en la forma de las matrices de reflexión de calzadas en autopistas argentinas. Cabe destacar que las tablas-r analizadas se obtuvieron mediante mediciones "in-situ" con reflectómetro de calzadas. Un primer grupo se corresponde con las condiciones iniciales de pavimentos macro texturados y fueron mensuradas en el período 2001/2003. El segundo contiene tablas-r del mismo tipo, con un uso tal que pueden considerarse superficies en estado estacionario, evaluadas entre los años 2005 y 2009.

A partir de estas experiencias, se busca aportar información acerca de la forma de las matrices y los modos en que se alteran, la especularidad y el factor de escala, relacionando tales parámetros con los posibles efectos en la iluminación resultante.

\section{2.- Cambios en la matriz de reflexión}

Un posible modelo para explicar cualitativamente el fenómeno de reflexión en las calzadas asigna a la superficie dos tipos de textura: una gruesa, perceptible al tacto o vista (macro textura) y otra fina e imperceptible, la micro textura. ${ }^{2}$ Esta última sería responsable de una reflexión del tipo regular o difusa; mientras que, la interacción con la rugosidad gruesa produciría direcciones preferenciales de reflexión, asociadas con el mayor o menor

${ }^{1}$ La relación 3:1 fue válida al momento de redacción de la primera versión del capítulo (2016/17). Actualmente, los precios locales de las luminarias LED resultan casi equiparable a una luminaria con lámpara de descarga.

2 De Boer, J., Cohu, M., Schreuder, D., Public Lighting. Philips technical Library, Países Bajos, 1967; De Boer, J., Van Bommel, W., Road Lighting, Philips technical Library, Países Bajos, 1980. 
grado de especularidad. La figura 71 esquematiza la superficie del pavimento según esta concepción.

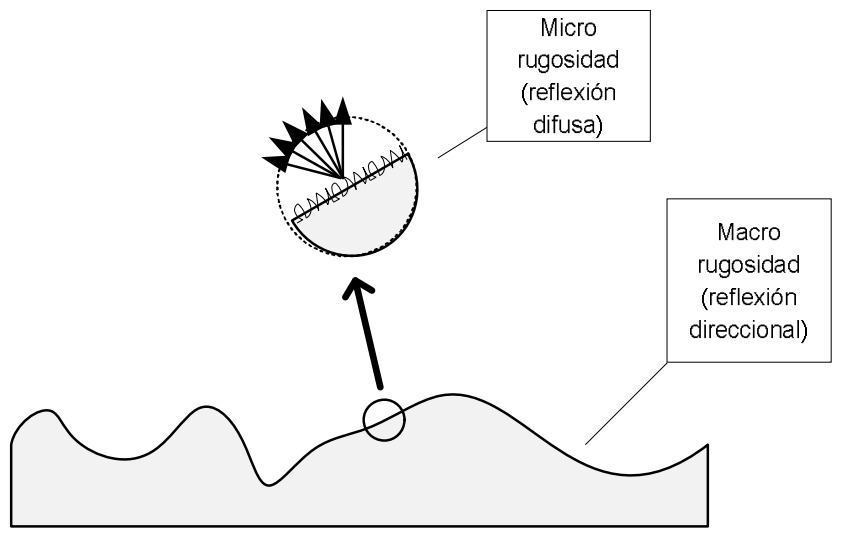

Figura 71. Textura de la superficie del camino. ${ }^{3}$

Este modelo explicativo es útil tan solo para describir el fenómeno, ya que no permite cuantificarlo. No obstante, a partir de él se puede deducir que al alisar la superficie, el tránsito disminuiría las macro rugosidades. De esta forma, el uso cambiaría la relación entre micro y macro texturas, favoreciendo el predominio de las primeras. La calzada evolucionaría entonces desde su estado inicial más especular, hacia otro, estacionario y más difuso. Sería así previsible que las uniformidades en luminancia (general, Uo y longitudinales, Ul) se incrementaran y la iluminación mejorara durante los primeros meses de uso. Se puede agregar, además, que el rodamiento dejaría sedimentos oscuros que, junto a las secuelas de la suciedad, reducirían la luz reflejada por el pavimento. Este efecto, sumado a la natural disminución del flujo luminoso en lámparas (depreciación de luminarias $^{4}$ ), haría decaer con el tiempo al valor medio de luminancia.

Los estudios para verificar la teoría anteriormente descripta han sido escasos y se remontan hacia fines de la década del '80. En su trabajo sobre caracterización de calzadas, Bodmann y Schmit ${ }^{5}$ estudiaron la evolución de los pavimentos de más de una decena de instalaciones en una región de Alemania. La investigación se prolongó por cerca de dos años y si bien los datos obtenidos no fueron concluyentes, se observó una disminución importante del factor especular S1 durante los primeros meses de uso, seguida por una estabilización final (figura 72).

\footnotetext{
${ }^{3}$ Imagen extraída de: Ixtaina, P., Armas, A., Bannert, B., "Use effects on the reflection of macro textured surfaces”, Journal of Applied Engineering Sciences, 6(19), 2016a, pp. 51-56.

${ }^{4}$ De Boer, J., Cohu, M., Schreuder, D., Public Lighting, op. cit.; De Boer, J., Van Bommel, W., Road Lighting, op. cit.; CIE, Calculation and measurement of luminance and illuminance in road lighting, Publicación 30-2 - TC 4.6, Francia, 1982.

5 Bodmann, H., Schmidt, H., "Road surface reflection and Road Lighting: Field investigations", Lighting Research and Technology, 21(4), 1989, pp. 159-170.
} 


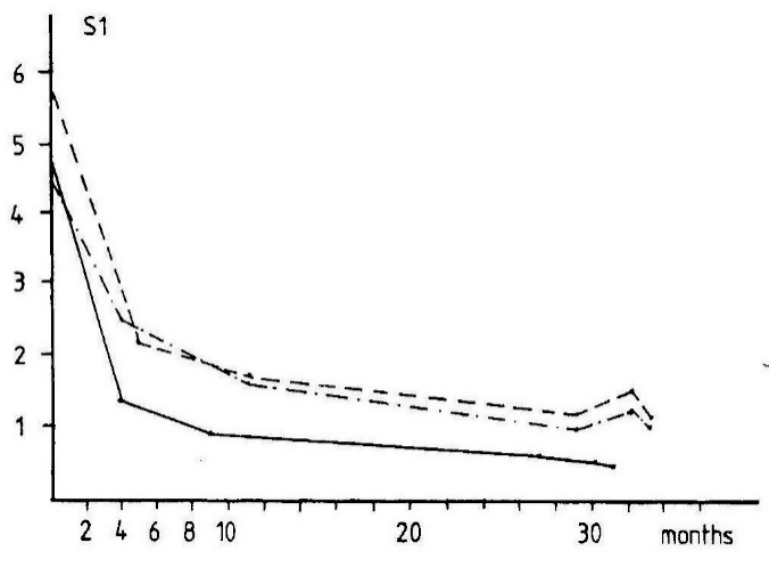

Figura 72. Evolución de S1. ${ }^{6}$

Las evaluaciones de las instalaciones efectuadas en el mismo trabajo fueron coherentes con esta baja en el grado de especularidad de los pavimentos, evidenciando una mejora de las uniformidades en luminancia (figura 73).
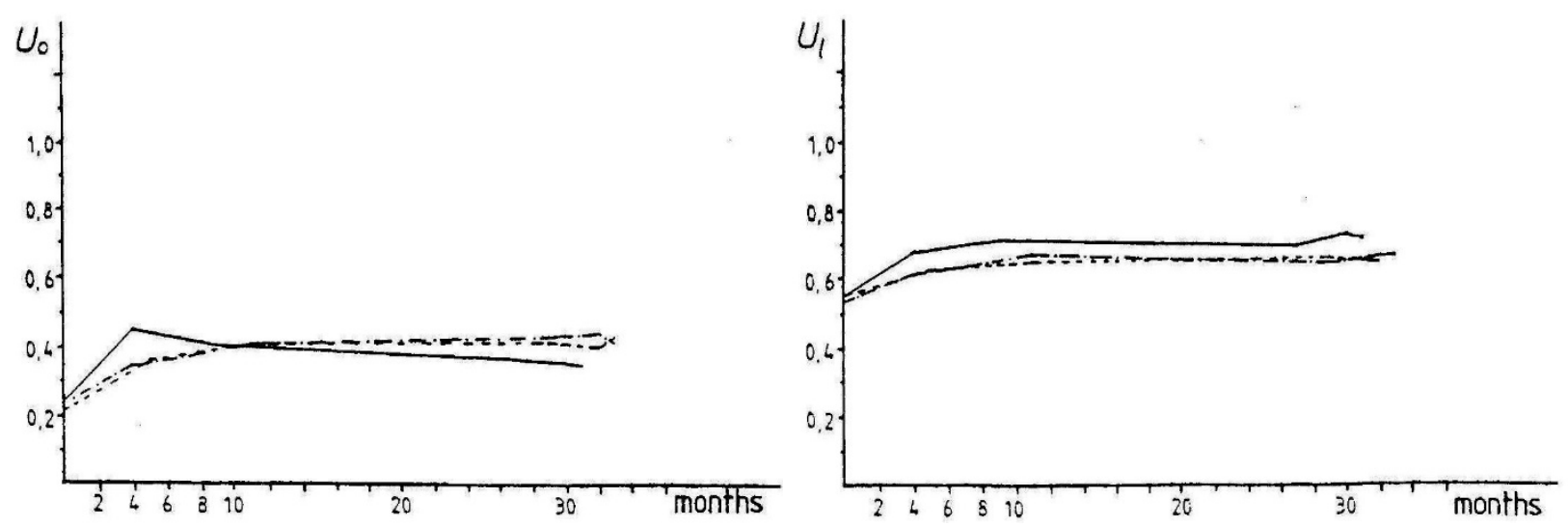

Figura 73. Evolución de las uniformidades. ${ }^{7}$

Asimismo, los estudios de Bodmann detectaron una caída del coeficiente de luminancia medio durante los primeros meses de uso, que puede asociarse directamente con una disminución global de la luz reflejada por el pavimento. En línea con este hecho, se observó la depreciación del valor medio de luminancia (figura 74).

${ }^{6}$ Ibídem.

${ }^{7}$ Ibídem. 

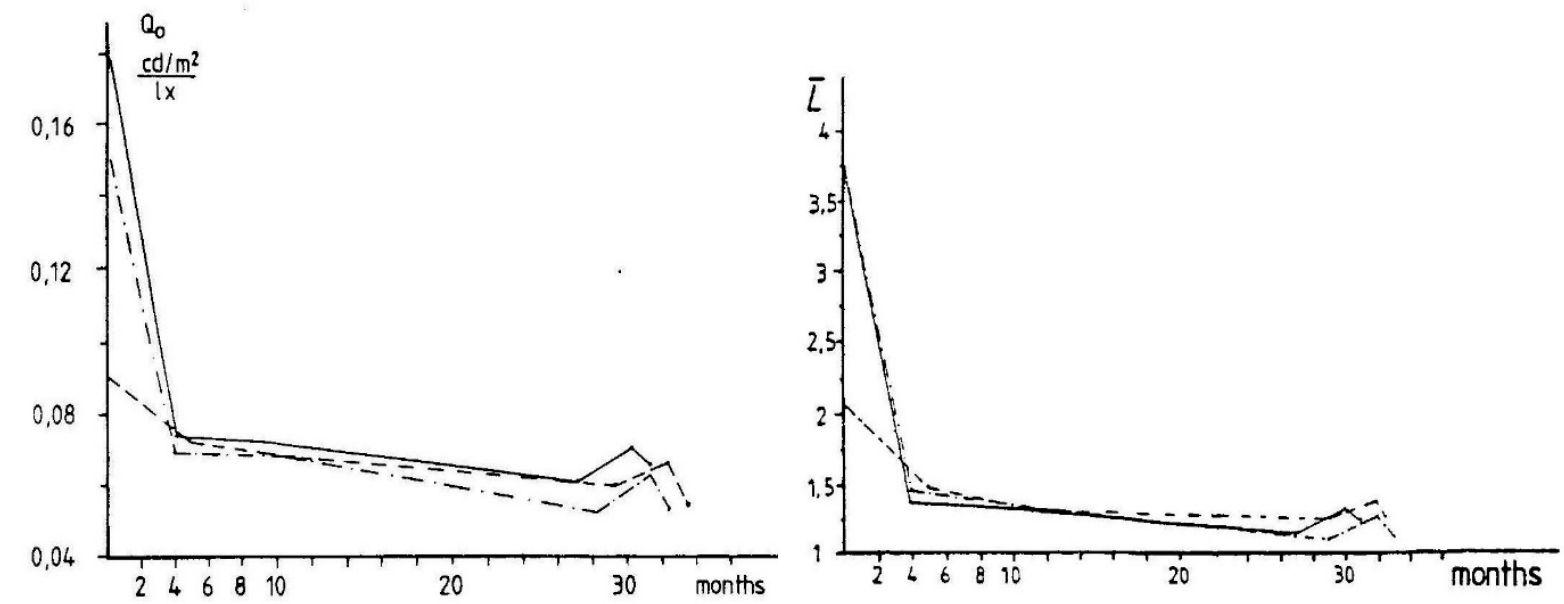

Figura 74. Evolución del coeficiente de luminancia medio y la luminancia media de la instalación. ${ }^{8}$

Finalmente, las superficies analizadas se estabilizaron dentro de un período máximo no mayor a seis meses. Estos resultados coincidieron con los de trabajos previos que, si bien abarcaron un período mayor de tiempo, involucraron un número no muy significativo de muestras. ${ }^{9}$

\section{3.- Estudios locales}

Se debe resaltar es que los resultados mostrados en el punto anterior difícilmente puedan extenderse a calzadas de locales (zona metropolitana, Argentina) dado que, por un lado, los estudios datan de hace casi 40 años, lapso que sin dudas implica cambios en las tecnologías para la formulación de los compuestos asfálticos, materiales y técnicas de aplicación. ${ }^{10}$ Por otro, los pavimentos de las autopistas locales son mayoritariamente del tipo "macro texturados" (drenantes), superficies que han sido las principalmente usadas en los últimos quince años.

Por lo dicho anteriormente, es necesario plantear que la tendencia de las investigaciones europeas no tendría razón para verificarse en nuestra región. En este sentido, los estudios realizados por el autor muestran tendencias en un sentido opuesto a las del Viejo Continente. ${ }^{11}$ Se realizó una evaluación semestral, durante tres años, de los parámetros de calidad según la Técnica de Luminancia (luminancia media, uniformidades general y longitudinal) y de Iluminancia. La investigación se inició en el momento de inauguración de la ruta, conjugando instalación de alumbrado y pavimento nuevos.

${ }^{8}$ Ibídem.

9 Ziegler, W., Reflexiontrockener und feuchterStrassenbelage Dissertation, Universitat Karlsruhe, 1980; Federal Highway Administration, Reflective characteristics of roadway pavements during wet weather, reporte FHWA/RD-86/104, 1986.

${ }^{10}$ El tema ya fue abordado en los capítulos precedentes, en donde se expuso material suficiente para demostrar que es altamente improbable que los materiales base empleados en una región de Europa en los '80 sean asimilables los aplicados en nuestra zona.

${ }^{11}$ Ixtaina, P., Armas, A., Bannert, B., "Use effects on the...”, op. cit.; Ixtaina, P. R., Vidal, P. A., "Changes with use and age of reflection properties of road surface new types", CIE Midterm Meeting and International Lighting Congress, León, 2005. 
Si bien las conclusiones muestran una paulatina disminución de los valores medios de luminancia (figura 75), la caída promedio fue notoriamente más suave que en el caso europeo. La uniformidad general Uo no presentó grandes cambios y sí fue notoria una baja importante de la uniformidad longitudinal con el uso, atribuible a un incremento de la especularidad de la calzada (figura 76).

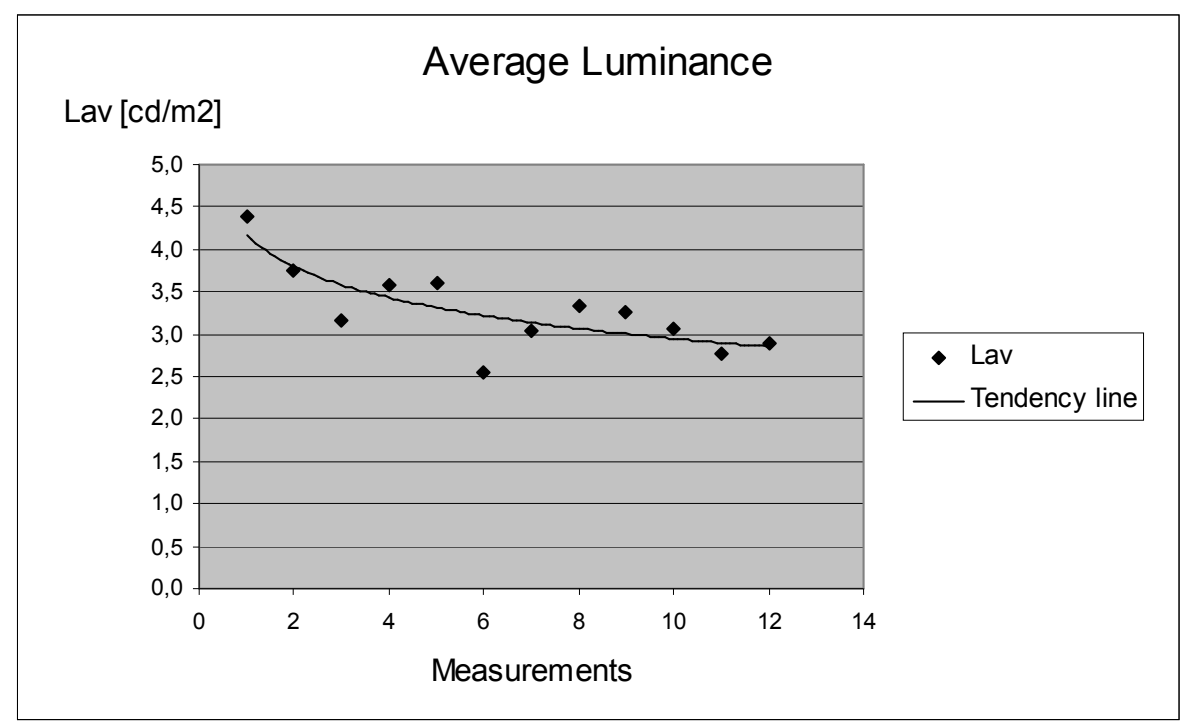

Figura 75. Evolución de la luminancia media. ${ }^{12}$

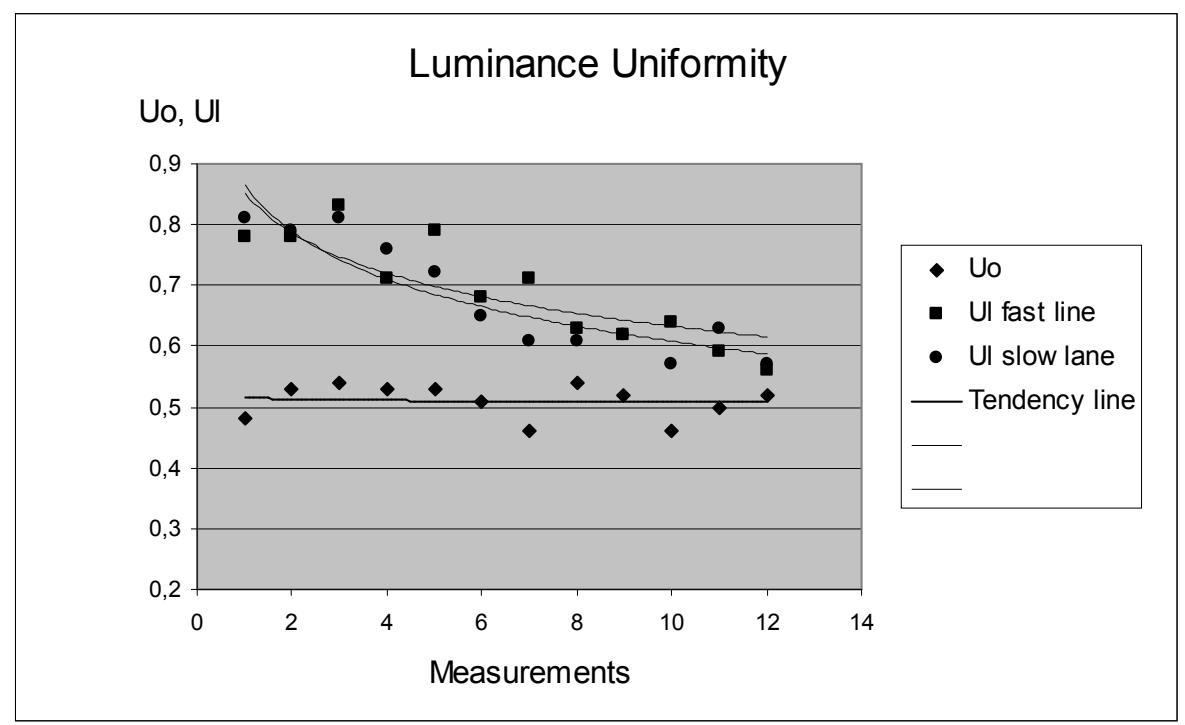

Figura 76. Evolución de las uniformidades. ${ }^{13}$

\section{4.- Medición de calzadas}

A modo de complemento del estudio anterior, se realizó para esta Tesis una evaluación directa de matrices de reflexión. Todas las tablas-r analizadas se obtuvieron mediante

${ }^{12}$ Imagen extraída de: Ixtaina, P. R., Vidal, P. A., “Changes with use and age of...”, op. cit.

${ }^{13}$ Ibídem. 
mediciones "in-situ", con reflectómetro de calzadas de acuerdo a lo descripto en el capítulo $3 .^{14}$

El primer grupo de calzadas con las que se trabajó se corresponde con las condiciones iniciales de los pavimentos macro texturados y fue medido en el período 2001/2003 en tres zonas de evaluación. Primero, la concesión de la llamada Autopista del Oeste, en este caso los tramos estudiados se ubicaron en las inmediaciones del $\mathrm{km} 60$, sentido a CABA (en la cercanías de Luján). Luego, las otras dos zonas estuvieron en la concesión de la Autopista del Sol, una sobre el acceso Norte, en la salida del peaje ruta 202 y sobre el Ramal Campana, inmediaciones del km 50.

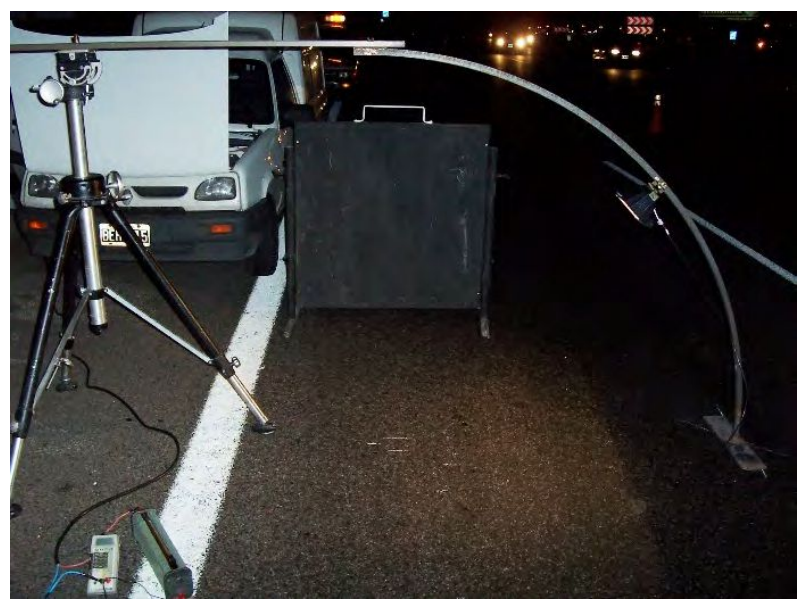

Figura 77. Montaje del Reflectómetro, autopista del Oeste (foto del autor).

Las tablas obtenidas, denominadas tipo HSI, coincidieron con las primeras aplicaciones locales de los nuevos compuestos "drenantes". Su estado puede considerarse como "inicial", ya que en ese momento contaban con entre cuatro meses (autopista del Oeste) y un año de uso (Acceso Norte). La evaluación incluyó una sección con pavimento en estado nuevo, prácticamente sin rodamiento.

La característica destacable de estas tablas-r es su alta especularidad que las torna no asimilables a las calzadas tradicionalmente consideradas para el diseño como R3 o R4 según el ya mencionado sistema CIE.

El segundo conjunto de evaluaciones se realizó por requerimiento de la empresa Autopistas Urbanas SA, AUSA, a cargo de la concesión de las autopistas que cruzan la ciudad de Buenos Aires y abarcan las trazas denominadas 25 de Mayo, Perito Moreno, Ilia, Av. Cantilo y Av. Lugones. El estudio abarcó un primer conjunto de mediciones sobre la autopista 25 de Mayo, en la zona de vínculo con la traza a La Plata (2005). El segundo (2009) fue más amplio: comprendió pruebas sobre las autopistas 25 de Mayo, km 8; Perito Moreno, inmediaciones del peaje Avellaneda; Ilia, sentido Centro (frente al circuito KDT) y 9 de Julio Sur (sobre calle Suárez). Las superficies obtenidas se denominaron tipo HSE, todas calzadas del tipo macro texturadas y con entre 3 y 8 años

\footnotetext{
${ }^{14}$ Vidal, P. A., Ixtaina, P. R., "Application of a New Pavement Reflectometer", 2nd Balkan Conference and Fair in Lighting Balkan-Light'02, Proceedings, Estambul, 2002; Ixtaina P., Vidal P., "Argentinean road surface characterization", International CIE Symposium on road surface photometric characteristics: Measurement Systems and Results, Torino, Italia, 2008.
} 
de uso, lo que permitió inferir que se encontraban en un estado que podría considerarse como estacionario.
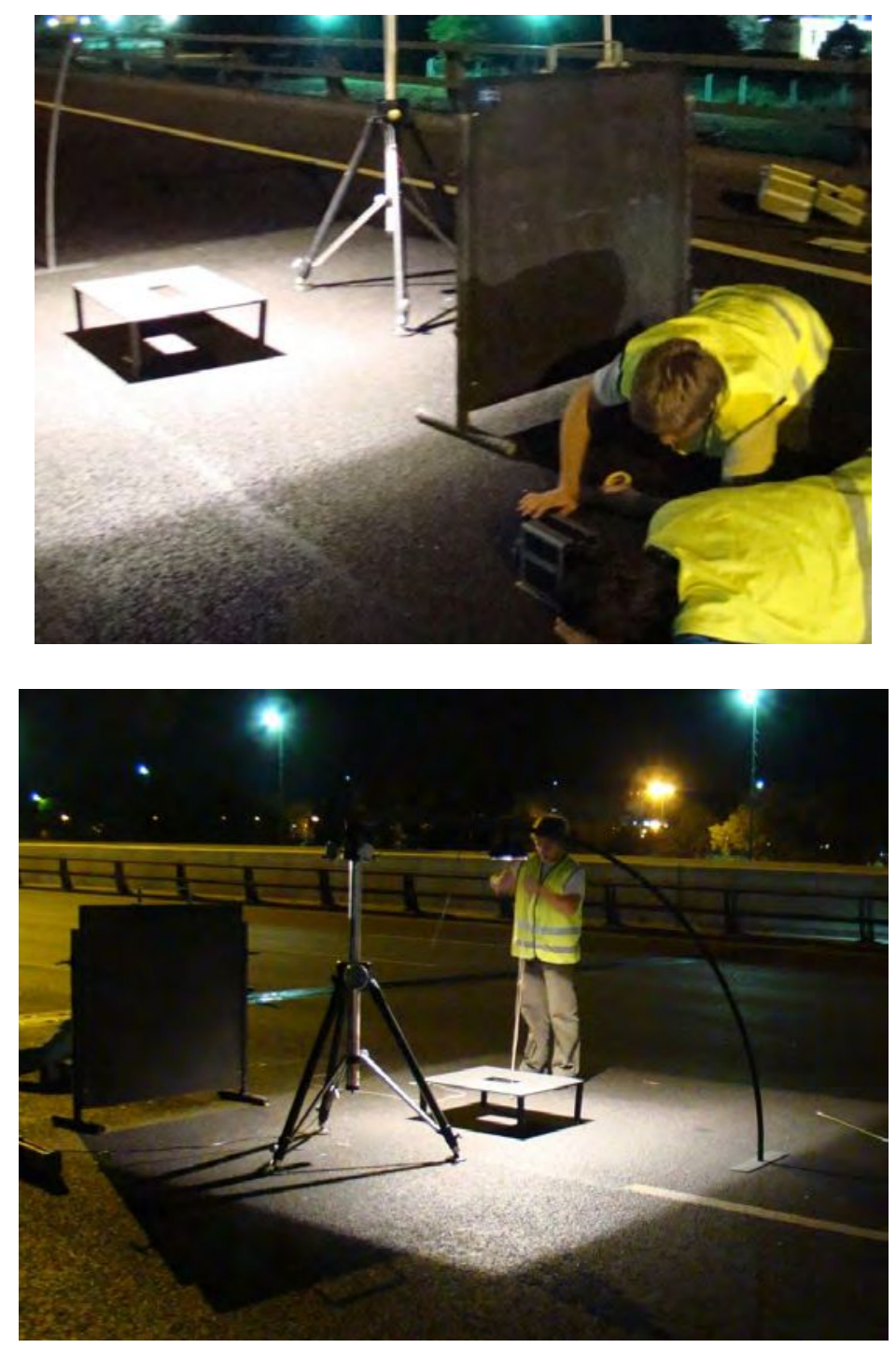

Figuras 78 y 79. Aspectos de la prueba en la autopista Ilía (fotos del autor).

\section{5.- Resultados}

\section{1- Superficies nuevas o con poco uso}

Estas son las denominadas HSI, basadas en seis sectores, con un total de casi veinte aplicaciones del reflectómetro. Las formas de reflexión pudieron agruparse en:

a) Superficies tipo la HSI-1, de muy alta especularidad, coincidente con las de menor rodamiento entre las estudiadas. El esquema espacial de reflexión y los valores típicos de los parámetros Qo, S1 y AD encontrados se indican en la figura 80. 


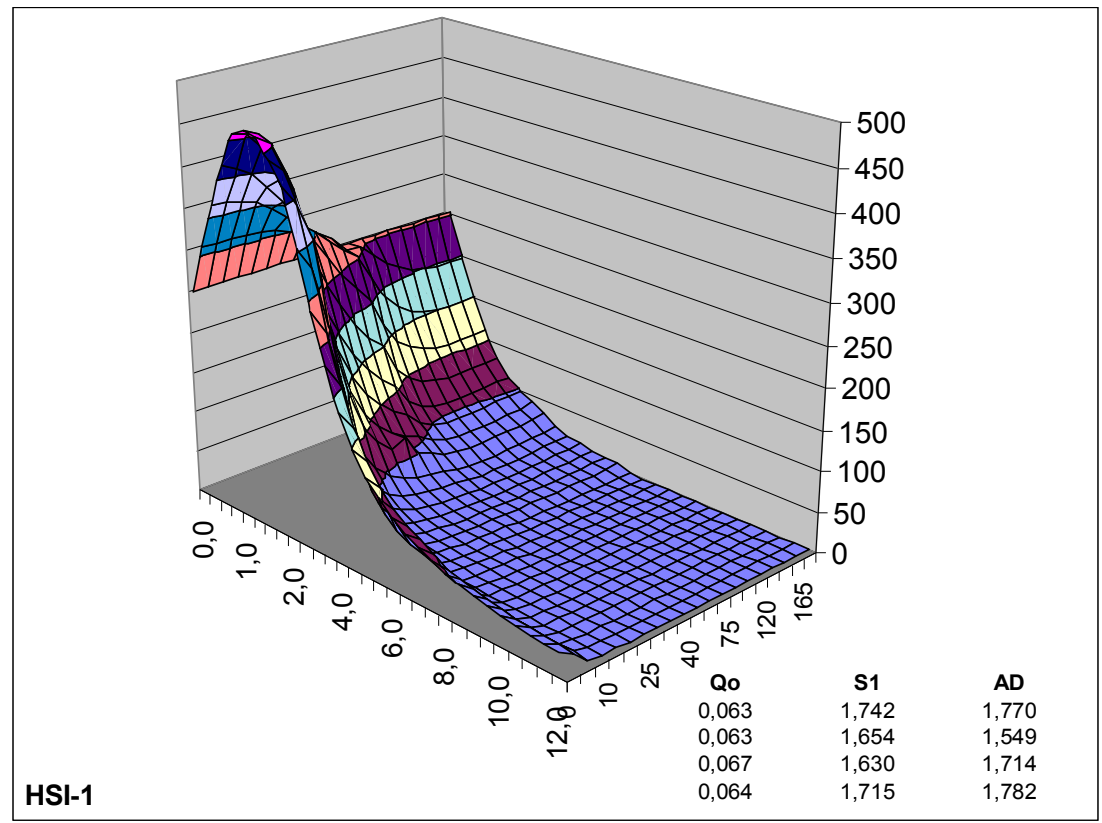

Figura 80. Gráfico representativo de pavimentos con poco rodamiento, HSI-1.

b) El segundo conjunto, HSI-2, con forma ligeramente más suave, se corresponde con pavimentos con cerca de un año de uso (figura 81).

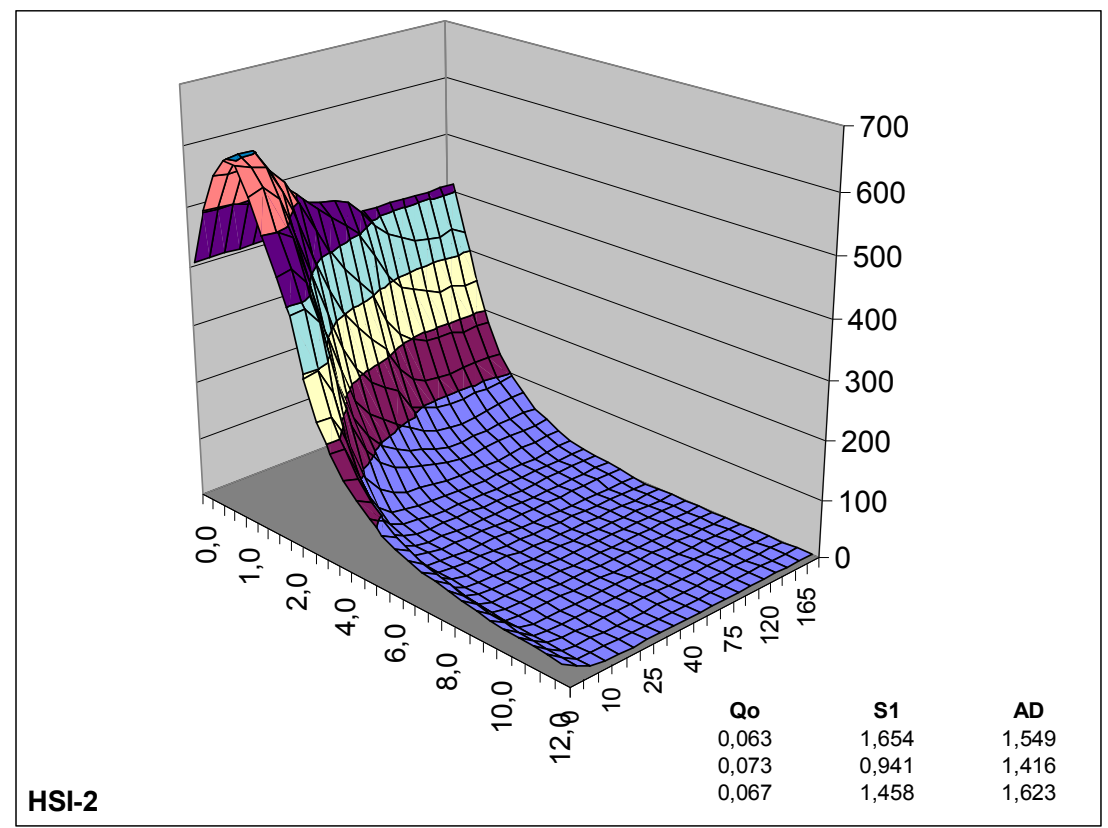

Figura 81. Gráfico representativo de pavimentos con casi un año de uso, HSI-2.

En la comparación entre ambos grupos se destacan dos cuestiones de interés: por un lado, hay una coincidencia en los valores de los coeficientes de luminancia media Qo, asimilable al concepto cualitativo de grado de claridad. Pese a ello, los pavimentos que se corresponden con la clasificación HSI-1, resultaban a simple vista, notoriamente más oscuros con, incluso, aspecto húmedo. La forma de la matriz posee un "pico" de reflexión 
más acentuado que la HSI-2, quizá responsable de su aspecto húmedo. Al mismo tiempo, ambas superficies deben considerarse como de alta especularidad, tal el sentido de los valores de $\mathrm{S} 1$ y $\mathrm{AD}$ cercanos a 1,5 - 1,6.

\section{2- Superficies con elevada carga de tránsito}

El estudio realizado entre 2005 y 2009 abarcó cerca de una decena de zonas de camino, con alrededor de 30 aplicaciones del reflectómetro. Las superficies fueron también concretos asfálticos macro texturados, $\mathrm{y}$, como se dijo, contaban con hasta ocho años de uso sin recapados o modificaciones en las zonas analizadas. La figura 82 muestra, a modo de ejemplo, una de las superficies encontradas (Av. 9 de Julio Sur). ${ }^{15}$

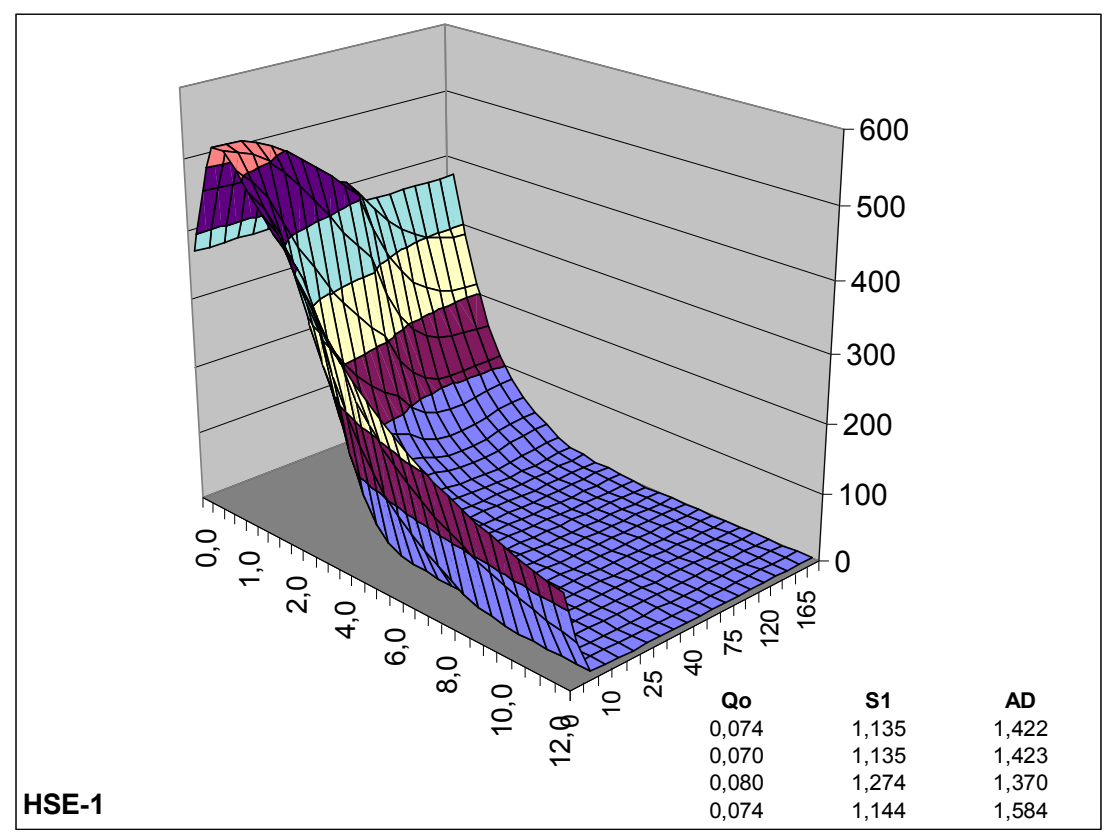

Figura 82. Tabla representativa de pavimentos en estado estacionario.

Como puntos característicos se observaron un leve incremento de Qo con respecto a las tablas obtenidas para superficies en estado inicial, lo que podría traducirse en una superficie levemente más clara.

Con respecto al grado de especularidad, la forma dista de ser "difusa". Si observamos el índice CIE (S1), sus valores son menores que en los pavimentos tipo HSI (S1 $1,1 / 1,2)$. Sin embargo, el índice del sistema LAL-CIC se mantiene elevado $(A D \approx 1,4 / 1,5)$ lo que indicaría un grado de especularidad no muy distinto que las calzadas en estado inicial estudiadas.

\section{6.- Comentarios finales}

${ }^{15}$ El detalle de esta investigación junto con la totalidad de resultados se encuentra publicado en: Ixtaina, P., Presso, "Estudio de Calzadas en las Autopistas Urbanas de la ciudad de Buenos Aires", Actas del X Congreso Panamericano de Iluminación, Valparaíso (Chile), octubre de 2010, pp. 108-113. 
El recorrido presentado en este capítulo permite dar cuenta de que en Argentina las variaciones en las calzadas provocadas por el uso y envejecimiento de los pavimentos no se da de la misma forma que expresan estudios anteriores realizados en el extranjero.

Se muestran evidencias de que es posible hablar de un "estado estacionario" para superficies con cerca de tres años de rodamiento. El período inicial de estabilización resultó más largo que el obtenido en las investigaciones europeas sobre pavimentos tradicionales (entre 4 y 6 meses). Asimismo, el hecho de obtener mediciones con resultados coincidentes dentro de lapsos de más de 4 años, permite hablar además de un estado estacionario que se mantendría por largos períodos.

A diferencia de las investigaciones de Bodmann et al, ${ }^{16}$ no se pude inferir el paso de una matriz más "especular" hacia otra más "difusa". Los resultados obtenidos indican que las superficies nuevas y usadas se mantienen prácticamente con la misma especularidad, pero alterándose la posición y forma del pico de reflexión. Posiblemente esto explique lo encontrado en el estudio previo del autor, ${ }^{17}$ esto es, el importante cambio en Ul con el uso sin una alteración significativa de Uo.

Como dato a destacar para diseñadores y proyectistas, las formas encontradas no son coincidentes con los estándares CIE R3 o R4, lo que remarca la importancia de emplear en los diseños tablas-r que representen correctamente las superficies de nuestra región. Esta constatación permite afirmar finalmente, que el cambio al que se refiere influye mucho en la variación de los parámetros lumínicos durante la vida de la instalación y resulta necesario particularizarlos lo mejor posible, teniendo en cuenta los contextos de fabricación y aplicación de la carpeta asfáltica.

${ }^{16}$ Bodmann, H., Schmidt, H., "Road surface reflection...", op. cit.

${ }^{17}$ Ixtaina, P. R., Vidal, P. A., "Changes with use and...”, op. cit. 
Capítulo 6 - Eficiencia, ahorro de energía y su vínculo con las propiedades de las calzadas 
Resulta importante reconocer que el acabado conocimiento de las propiedades reflectivas de las calzadas conduce a diseños más exactos, potenciando con ello las posibilidades de obtener el nivel justo (normalizado) de alumbrado y de optimizar así el consumo energético. También es fácil observar que pavimentos "claros" reflejarán más la luz, aumentarán la luminancia media de la calzada y mejorarán le eficiencia energética de la instalación. Todos estos conceptos no son nuevos y de hecho existen antecedentes del agregado de "aclarantes" a los compuestos asfálticos para consumir menos energía. ${ }^{1}$

El presente capítulo se concentrará en las particularidades de las superficies de autopistas de nuestra zona que fueron estudiadas. Los abordajes que se describirán demuestran las posibilidades ciertas de un 30\% de ahorro energético, si el pavimento se escoge adecuadamente.

Antes, resulta conveniente introducir algunos conceptos básicos que atañen al comportamiento de un sistema de alumbrado vial, tanto desde el punto energético como de los parámetros lumínicos resultantes. Se asume, entonces, que la instalación de alumbrado artificial está destinada a brindar las mejores condiciones de percepción a los conductores de vehículos en calles o rutas, con un mínimo consumo energético. ${ }^{2}$

\section{1.- Caracterización del consumo energético en instalaciones de alumbrado vial}

\section{1.- Densidad de potencia}

La densidad de potencia $\mathrm{P}_{\mathrm{D}}$ describe la potencia instalada por unidad de área de camino. Si P es la potencia por luminaria (potencia de fuente luminosa-lámpara o LEDmás la del equipo auxiliar), $\mathrm{P}_{\mathrm{D}}$ se define como $[3,4]$ :

$$
P_{D}=\frac{P}{A}=\frac{P}{S a_{N}}
$$

El área elemental (A) es la porción de calzada entre columnas: ancho de calzada $\left(\mathrm{a}_{\mathrm{N}}\right)$ por separación entre luminarias $(\mathrm{S}$, figura 83$)$.

\footnotetext{
${ }^{1}$ De Boer, J., Cohu, M., Schreuder, D., Public Lighting. Philips technical Library, Países Bajos, 1967.

${ }^{2}$ De Boer, J., Cohu, M., Schreuder, D., Public Lighting, op. cit.; Instituto Argentino de Racionalización de Materiales, IRAM AADL J 2022-2, Alumbrado Público, Vías de Tránsito - Clasificación y Niveles de Iluminación, Buenos Aires, 1995.
} 


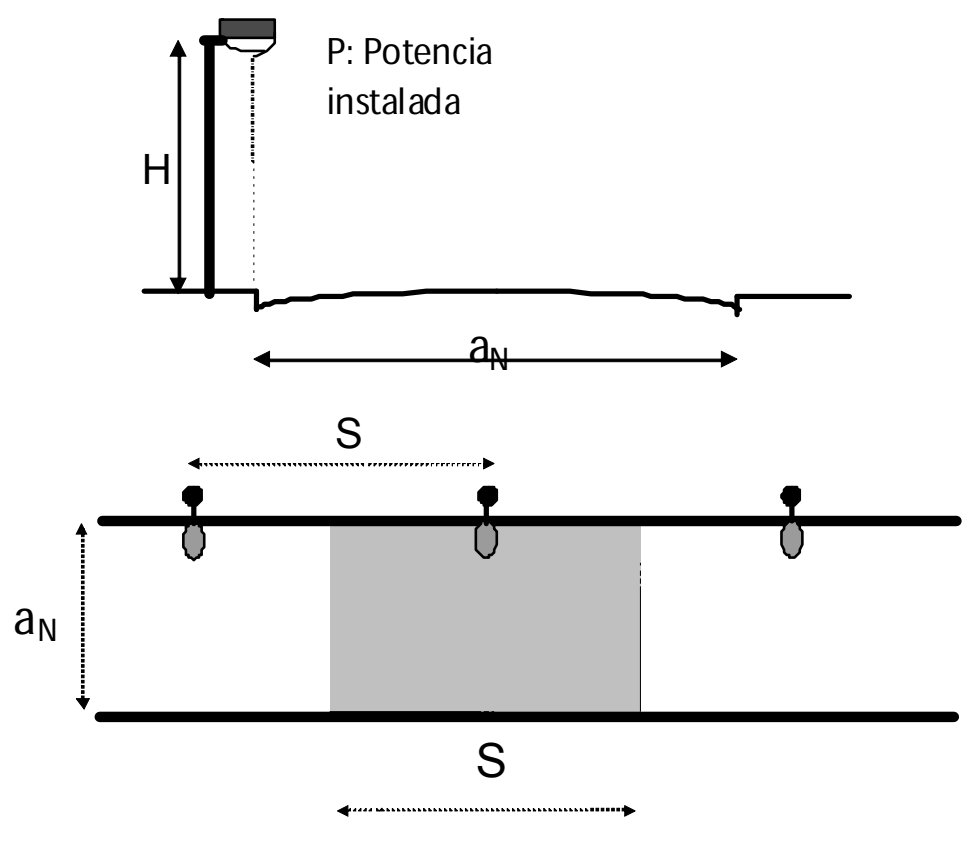

Figura 83. Esquema elemental de la calzada. ${ }^{3}$

\section{2.- Densidad de potencia normalizada}

Para cuantificar la densidad de potencia en forma relativa al nivel de alumbrado producido, se define la densidad de potencia normalizada $\mathrm{P}_{\mathrm{N}}$ :

$$
P_{N}=\frac{P_{D}}{L_{m}}=\frac{P}{S a_{N} L_{m}}
$$

En la ecuación (2), $\mathrm{L}_{\mathrm{m}}$ es la luminancia media sobre la calzada, quedando expresada la densidad normalizada en $\mathrm{W} / \mathrm{m}^{2} / \mathrm{cd} / \mathrm{m}^{2}$.

\section{3.- Factor de utilización y pérdidas}

El rendimiento de la luminaria en la instalación $(\mu \mathrm{u})$ es uno de los términos básicos en el cálculo de sistemas de alumbrado. Con él es posible estimar la cantidad de flujo luminoso emitido por las luminarias $\left(\phi_{\text {Lum }}\right)$, que llega realmente a la calzada $\left(\phi_{\mathrm{u}}\right)$ :

$$
\mu \mathrm{u}=\frac{\phi_{\mathrm{u}}}{\phi_{\mathrm{Lum}}}
$$

Por otro lado, el flujo emitido por la luminaria dependerá lógicamente del flujo de la lámpara y de cuánto "retenga" de éste la luminaria, o sea, su rendimiento, conocido como LOR (LOR, luminary output radio):

$$
\mathrm{LOR}=\frac{\phi_{\text {Lum }}}{\phi \mathrm{Lamp}}
$$

\footnotetext{
${ }^{3}$ Esquema extraído de: Ixtaina, P., et al, “Argentinean Experience in Highways Led Lighting”, Energy and Environment Research, 7(1), 2017, pp. 31-37.
} 
De este modo, el flujo que llega a la calzada $\left(\phi_{\mathrm{u}}\right)$ puede expresarse como:

$$
\phi \mathrm{u}=\mu \mathrm{u} \operatorname{LOR} \phi \mathrm{Lamp}
$$

Por otro lado, parte del flujo emitido por la luminaria ( $\phi$ Lum) no llegará a la calzada, lo que puede considerarse como pérdidas $\left(\phi_{\mathrm{p}}\right)$ :

$$
\phi_{\mathrm{p}}=\phi_{\mathrm{Lum}}-\phi_{\mathrm{u}}
$$

Definiendose el coeficiente de pérdidas k como:

$$
\phi_{\mathrm{p}}=\mathrm{k} \phi_{\mathrm{u}}
$$

\section{4.- Coeficiente de luminancia}

Tal como se vio en el capítulo 3, la luminancia $L$ de una superficie elemental $\Delta S$ sobre la calzada (figura 84), queda determinada por la ecuación (7):

$$
\mathrm{L}=\frac{\mathrm{I}(\mathrm{C}, \gamma)}{\mathrm{H}^{2}} \mathrm{q}(\alpha, \beta, \delta, \gamma) \cos ^{3}(\gamma)
$$

$\mathrm{I}(\mathrm{C}, \gamma)$ es la intensidad luminosa de la luminaria en dirección al punto en el que se calcula la luminancia, $\mathrm{H}$ la altura de montaje de la luminaria y $\mathrm{q}$ es el coeficiente de luminancia del pavimento.

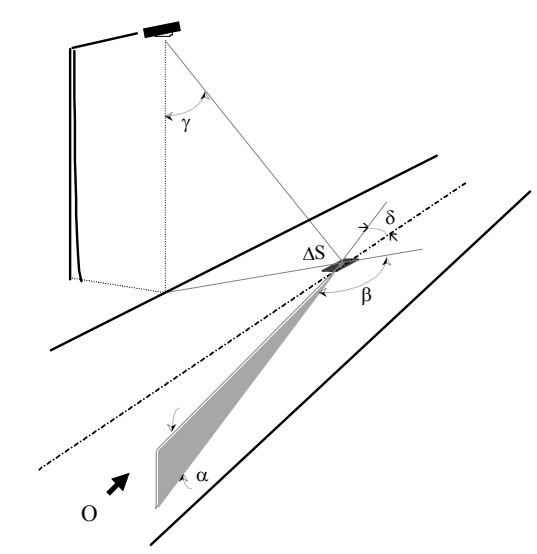

Figura 84. Geometría básica para el análisis de la visión en rutas. ${ }^{4}$

Si E es la iluminancia puntual sobre la calzada, la ecuación (8) puede reescribirse como:

$$
\mathrm{L}=\mathrm{q}(\beta ; \gamma) \mathrm{E}
$$

El coeficiente de luminancia cumple la función de factor de proporcionalidad, para cada punto de la calzada, entre iluminancia y luminancia. Con este sentido, se define el

\footnotetext{
${ }^{4}$ De Boer, J., Cohu, M., Schreuder, D., Public Lighting, op. cit.; CIE, Calculation and measurement of luminance and illuminance in road lighting, Publicación 30-2 - TC 4.6, Francia, 1982.
} 
coeficiente de luminancia medio Qo, que cuantifica el grado de "claridad" de la superficie del camino: ${ }^{5}$

$$
\mathrm{Q}_{0}=\frac{1}{\Omega_{0}} \int_{\Omega_{0}} \mathrm{qd} \Omega
$$

En la ecuación $10, \Omega$ representa el ángulo sólido que subtiende al elemento $\Delta \mathrm{s}$ de la figura 84. Mayores valores de Qo, asociados con pavimentos más "claros", permitirán obtener un incremento de la luminancia media, para un mismo sistema de iluminación.

Como se discutió en el capítulo 3, el cálculo del coeficiente de luminancia medio requiere de la tabla-r real del pavimento, que por lo general no es conocida. Sin embargo, si se cuenta con una cantidad suficiente de evaluaciones simultáneas de luminancias e iluminancias puntuales en varias secciones de una vía de tránsito, es posible utilizar el factor q00 -relación entre luminancia media e iluminancia media-, como una aproximación empírica al grado de claridad de la calzada: ${ }^{6}$

$$
\mathrm{q}_{00}=\frac{\mathrm{Lm}}{\mathrm{Em}}
$$

Debe quedar claro que no existe ninguna relación teórica entre las definiciones de $\mathrm{Q}_{0}$ y q00. Pese a ello, la baja dispersión obtenida en el análisis de un número importante de evaluaciones, permite suponer un buen desempeño de este coeficiente como indicador del grado de claridad de la calzada. ${ }^{7}$

Combinando las expresiones (5), (9) y (11), podemos expresar la relación final que existe entre la luminancia que obtendremos sobre la calzada y la potencia consumida por la luminaria:

$$
L=q_{00} \mu_{u} \frac{L O R \eta_{L} \eta_{B} P_{L u m}}{S a_{N}}
$$

En (12), $\mu \mathrm{u}$ es el rendimiento de la luminaria en la instalación ya mencionado, $\mathrm{q}_{00}$ el coeficiente de luminancia media, LOR es el rendimiento de la luminaria, $\eta \mathrm{L}$ es la

\footnotetext{
${ }^{5}$ Keitz, H. A. E, Light calculations and measurements, N. V. Philips, Holanda, 1974; van Bommel, W., de Boer, J., Road Lighting, Philips Technical Library, Países Bajos, 1982; Erbay, A., "Reflection properties of road surfaces", ILTUB, Berlin, 1974b.

${ }^{6}$ Ixtaina, P. et al, "Effects of led lighting on the luminance coefficient", Light \& Engineering, 25(4), 2017a, pp. 115-122; Ixtaina, P., Vidal, P., "Road surface lightness and energy efficiency in road lighting", Proceedings of the 12th European Lighting Conference "LUX EUROPA 2013", Cracovia (Polonia), 2013. ${ }^{7}$ Ibídem.
} 
eficacia de la lámpara, $\eta \mathrm{B}$ es el rendimiento del balasto y PLum la potencia de la luminaria.

\section{5.- Eficiencia en instalciones viales: valores típicos y esperables}

En el trabajo ya citado, P. Pracki ${ }^{8}$ elaboró una propuesta de clasificación energética para instalaciones de alumbrado vial. Su estudio se basó en la simulación, mediante software, de varias alternativas de instalación. Las combinaciones logradas generaron un rango de valores posibles de $\mathrm{P}_{\mathrm{D}}$ y $\mathrm{P}_{\mathrm{N}}$ que posteriormente se utilizaron para calificar energéticamente a la instalación.

Las tablas 7 y 8 , extraídas de la referencia citada, sintetizan la propuesta de clasificación.

\begin{tabular}{|c|c|c|}
\hline \multicolumn{2}{|c|}{ Clase de eficiencia energética } & $\mathbf{P}_{\mathbf{N}}\left[\mathbf{W} / \mathbf{m}^{2} / \mathbf{c d} / \mathbf{m}^{\mathbf{2}}\right]$ \\
\hline A & La mayor eficiencia energética & $<0,2$ \\
\hline B & Muy eficiente & $0,2-0,4$ \\
\hline C & Eficiente & $0,4-0,6$ \\
\hline D & Eficiencia intermedia & $0,6-0,8$ \\
\hline E & Poco eficiente & $0,8-1,0$ \\
\hline F & Muy poco eficiente & $1,0-1,2$ \\
\hline G & La menor eficiencia energética & $>1,2$ \\
\hline
\end{tabular}

Tabla 7. Clasificación en función de la potencia normalizada. ${ }^{9}$

\begin{tabular}{|c|c|c|c|c|c|c|}
\hline \multirow{2}{*}{ Clase } & \multicolumn{7}{|c|}{ Po $\left[\mathbf{W} / \mathbf{m}^{\mathbf{2}}\right]$} \\
\cline { 2 - 7 } & ME1 & ME2 & ME3 & ME4 & ME5 & ME6 \\
\hline A & $<0,4$ & $<0,3$ & $<0,2$ & $<0,15$ & $<0,1$ & $<0,06$ \\
\hline B & $0,4-0,8$ & $0,3-0,6$ & $0,2-0,4$ & $0,15-0,3$ & $0,1-0,2$ & $0,06-0,12$ \\
\hline C & $0,8-1,2$ & $0,6-0,9$ & $0,4-0,6$ & $0,3-0,45$ & $0,2-0,3$ & $0,12-0,18$ \\
\hline D & $1,2-1,6$ & $0,9-1,2$ & $0,6-0,8$ & $0,45-0,6$ & $0,3-0,4$ & $0,18-0,24$ \\
\hline E & $1,6-2,0$ & $1,2-1,5$ & $0,8-1,0$ & $0,6-0,75$ & $0,4-0,5$ & $0,24-0,30$ \\
\hline F & $2,0-2,4$ & $1,5-1,8$ & $1,0-1,2$ & $0,75-0,9$ & $0,5-0,6$ & $0,30-0,36$ \\
\hline G & $>2,4$ & $>1,8$ & $>1,2$ & $>0,9$ & $>0,6$ & $>0,36$ \\
\hline
\end{tabular}

Tabla 8. Clasificación en función de la densidad de potencia. ${ }^{10}$ la CIE. ${ }^{11}$

La clasificación de la tabla 8 , se basa en las exigencias de alumbrado que plantea

La clasificación anterior fue elaborada teniendo en cuenta las eficacias típicas de lámparas convencionales: incandescente, mercurio alta presión, sodio alta y baja presión, y halogenuros metálicos. La eficacia de la lámpara se articuló con distintas posibilidades

\footnotetext{
${ }^{8}$ Pracki, P., “A proposal to classify road lighting energy efficiency”, Lighting Research and Technology, 43, 2011, pp. 271-280.

9 Tabla extraída de: Pracki, P., “A proposal to classify...”, op. cit.

${ }^{10}$ Ibídem.

11 International Organization for Standardization ISO, "Guide to the expression of uncertainty in measurement", Suiza, 1993.
} 
de configuración del sistema de alumbrado, combinando los esquemas de montaje típicos (por ejemplo, columnas en un lateral, en el centro de una calzada de doble vía, etc.) con diseños realizados respetando conceptos de "eficiencia energética": luminarias apantalladas (para evitar emisiones por fuera del área a iluminar), fotometrías acordes a la geometría del trazado, etc.). El estudio no incorporó luminarias LED, que recién comenzaban a instalarse en la fecha de publicación del trabajo. Como se verá en puntos posteriores, la eficacia actual de tales dispositivos debería modificar la clasificación de Pracki.

\section{2.- Eficiencia en instalaciones argentinas}

El presente punto toma como base las investigaciones realizadas por el autor como parte de su actividad científica dentro del LAL - CIC. Estas tareas tienen como base la evaluación de instalaciones viales de autopistas, actividad que posibilitó recabar información real sobre sistemas de alumbrado diseñados y evaluados por la Técnica de Luminancia. Se efectuaron a partir de un Convenio entre la CIC y el OCCOVI (Organismo de Control de Concesiones Viales), vigente desde mediados de 2001 y han permitido contar con acceso a los diferentes corredores viales con personal técnico y de seguridad vial.

La gran mayoría de los diseños por luminancia concretados en el país se concentran en la Red de Accesos a la ciudad de Buenos Aires, que comprende cuatro concesiones viales: Acceso Norte, con dos ramales principales, Acceso Oeste, Autopista Ezeiza Cañuelas como ingreso sur y finalmente la Autopista La Plata Buenos Aires, que vincula la capital nacional con la provincial. Esta red de autopistas, cuya traza íntegra posee iluminación artificial, se completa con las también iluminadas Autopistas Urbanas de la Ciudad de Buenos Aires, que la cruzan, y la Avenida General Paz, que la circunda. El conjunto involucra alrededor de $400 \mathrm{~km}$ de autopistas cuyos sistemas de alumbrado se encuentran en la actualidad en diversas etapas de reconversión a tecnología LED.

La red está compuesta por instalaciones típicas de autopista, con reserva central (cantero o división tipo "New Jersey"), entre dos y seis carriles por mano y vanos de entre 50 y hasta $65 \mathrm{~m}$. Las alturas de montaje de las luminarias rondan los $16 \mathrm{~m}$ y en la mayoría de los casos los niveles de alumbrado se obtienen con cuatro luminarias por columnas, orientadas dos para cada sentido de circulación. La iluminación se rige por la normativa nacional ya mencionada en el capítulo 2: IRAM AADL J 2022-2, que sigue a la Recomendación CIE 30.2.

\section{1.- Parámetros típicos. Instalaciones con lámparas de descarga.}

El uso de luminarias equipadas con lámparas de sodio alta presión, $400 \mathrm{~W}$ de potencia, fue la regla para los diseños originales de las instalaciones de alumbrado descriptas, que se diseñaron y construyeron desde 1996 (Acc. Norte y Oeste) hasta 2000/2001 (tramo Hudson - La Plata de la autopista La Plata-Buenos Aires. El mismo tipo de luminarias se usó en las autopistas urbanas, construidas a mediados de la década del '80 (las cuales fueron objeto de planes de mantenimiento /recambio de lámparas y limpieza de luminarias) y a mediados de los '90. Las autopistas fueron consideradas calzadas tipo A, estableciéndose $2,7 \mathrm{~cd} / \mathrm{m} 2$ como luminancia media inicial, uniformidad general Uo mayor a 0,4 y uniformidades longitudinales mayores a 0,7 . 
En instalaciones con alturas de montaje considerable, del orden de $16 \mathrm{~m}$, como es el caso de las autopistas argentinas, una parte importante de la luz emitida por las luminarias alcanza las banquinas y zonas aledañas. En tales casos, las pérdidas son altas y por ende $\mathrm{k}$ (ver ecuación 7) dificilmente resulta menor a la unidad. Con respecto al rendimiento de la luminaria usada, $\mathrm{LOR}=0,75$ fue el valor mínimo admitido por el programa PRONUREE de Argentina para el financiamiento de instalaciones de alumbrado. ${ }^{12} \mathrm{LOR}=0,75$ es un rendimiento típico para refractores de vidrio prismardo del tipo de los empleados hasta el año 2000. Valores más altos se obtuvieron en luminarias con ópticas cuidadas y cierres transparentes (policarnobatos o vidrios planos transparentes), hasta valores en el rango $0,78-0,82$, típico de las últimas instalaciones diseñadas con tecnología de lámparas de descarga.

La tabla 9 muestra los parámetros energéticos de las luminarias empleadas en esta etapa y la tabla 10 resume la eficacia global resultante con estos equipos.

\begin{tabular}{|l|c|}
\hline Luminarias - LOR & $0,75-0,85$ \\
\hline Lámpara $-\eta_{\mathrm{L}}$ & $120 \mathrm{~lm} / \mathrm{W}-140 \mathrm{~lm} / \mathrm{W}$ \\
\hline Balasto $-\eta_{\mathrm{B}}$ & $0,90-0,95$ \\
\hline
\end{tabular}

Tabla 9. Parámetros típicos de luminarias convencionales.

\begin{tabular}{|c|l|}
\hline LOR $\mu_{\mathbf{L}} \mu_{\mathbf{B}}$ & \\
\hline $0,75 \times 120 \mathrm{~lm} / \mathrm{W} \times 0,90$ & $81 \mathrm{~lm} / \mathrm{W}$ \\
\hline $0,85 \times 140 \mathrm{~lm} / \mathrm{W} \times 0,95$ & $113 \mathrm{~lm} / \mathrm{W}$ \\
\hline
\end{tabular}

Tabla 10. Eficacias para luminarias convencionales.

En cuanto a eficacias de lámparas, $120 \mathrm{~lm} / \mathrm{W}$ corresponde a una lámpara de sodio alta presión de uso corriente, disponible comercialmente desde mediados de los '90. 140 $\mathrm{lm} / \mathrm{W}$ es un valor típico para lámparas SAP de última generación (conocidas comunmente como sodio Plus o sodio mejorado) y que surgieron entre 2001 y 2003. También estos valores son representativos de la tecnología denominada "sodio cerámico", de luz blanca. Estas últimas alcanzaron valores cercanos a $160 \mathrm{~lm} / \mathrm{W}$, según consta en pruebas realizadas en instalaciones entre 2010 y 2011. ${ }^{13}$ Pese a su alta eficacia, fueron desplazadas comercialmente por el surgimiento de la tecnología LED en el alumbrado público.

La tabla 11 resume resultados energéticos de instalaciones evaluadas entre 2006 y 2010, que se corresponden con los corredores viales ya mencionados; se agrupan aquellas secciones con geometría y tipo de instalación similares. Los datos mostrados son promedios para aquellas secciones que contaban además con un número mínimo de cuatro evaluaciones en zonas aledañas. Todas las instalaciones poseían lámparas de vapor de

\footnotetext{
${ }^{12} \mathrm{https}$ //www.argentina.gob.ar/energia/ahorro-y-eficiencia-energetica/edificaciones-sectorpublico/alumbrado-publico-eficiente

${ }^{13}$ Ixtaina, P., et al, "Argentinean Experience in...", op. cit.; Ixtaina, P., Vidal, P., Ferreyra, "Eficiencia energética en instalaciones de alumbrado vial". Memorias del XI Congreso Iberoamericano de Iluminación “LUXAMÉRICA 2012”, Cartagena (Colombia), octubre de 2012, pp. 159-163.
} 
sodio alta presión y no fue posible determinar si se correspondían con la tecnología tradicional o mejorada.

\begin{tabular}{|c|c|c|c|c|c|c|c|}
\hline Instalación & $\begin{array}{c}\text { Altura } \\
\mathbf{d e} \\
\text { montaje }\end{array}$ & $\begin{array}{c}\mathbf{S x A}_{\mathbf{N}} \\
\text { [m2] }\end{array}$ & $\begin{array}{c}\text { Potencia } \\
\mathbf{d e} \\
\text { lámpara }\end{array}$ & $\begin{array}{c}\text { Potencia } \\
\text { total }\end{array}$ & $\begin{array}{c}\mathbf{L m} \\
\text { [cd/m2] }\end{array}$ & $\begin{array}{c}\mathbf{P}_{\mathbf{N}} \\
\text { [W/cd] }\end{array}$ & $\begin{array}{c}\mathbf{P}_{\mathbf{N}} \\
\text { [W/cd] } \\
\text { [nicial } \\
\text { estimada) }\end{array}$ \\
\hline $\begin{array}{c}\text { Central, dos } \\
\text { luminarias por } \\
\text { columna }\end{array}$ & $12 \mathrm{~m}$ & 399 & $400 \mathrm{~W}$ & $440 \mathrm{~W}$ & 4,34 & 0,25 & 0,20 \\
\hline $\begin{array}{c}\text { Central, cuatro } \\
\text { luminarias por } \\
\text { columna }\end{array}$ & $16 \mathrm{~m}$ & 945 & $400 \mathrm{~W}$ & $880 \mathrm{~W}$ & 2.32 & 0,40 & 0,32 \\
\hline $\begin{array}{c}\text { Central, dos } \\
\text { luminarias por } \\
\text { columna }\end{array}$ & $14 \mathrm{~m}$ & 420 & $400 \mathrm{~W}$ & $440 \mathrm{~W}$ & 2,74 & 0,38 & 0,30 \\
\hline $\begin{array}{c}\text { Central, cuatro } \\
\text { luminarias por } \\
\text { columna }\end{array}$ & $16 \mathrm{~m}$ & 840 & $400 \mathrm{~W}$ & $880 \mathrm{~W}$ & 2,16 & 0,49 & 0,39 \\
\hline $\begin{array}{c}\text { Central, cuatro } \\
\text { luminarias por } \\
\text { columna }\end{array}$ & $16 \mathrm{~m}$ & 864 & $400 \mathrm{~W}$ & $880 \mathrm{~W}$ & 3,37 & 0,37 & 0,30 \\
\hline $\begin{array}{c}\text { Central, dos } \\
\text { luminarias por } \\
\text { columna }\end{array}$ & $16 \mathrm{~m}$ & 420 & $600 \mathrm{~W}$ & $660 \mathrm{~W}$ & 3,38 & 0,46 & 0,37 \\
\hline $\begin{array}{c}\text { Central, cuatro } \\
\text { luminarias por } \\
\text { columna }\end{array}$ & $16 \mathrm{~m}$ & 385 & $400 \mathrm{~W}$ & $880 \mathrm{~W}$ & 3,08 & 0,74 & 0,60 \\
\hline
\end{tabular}

Tabla 11. Eficacias de instalaciones con lámparas de descarga.

Al momento de su evaluación todas las instalaciones estaban "depreciadas", es decir, contaban con al menos 4 años de uso o desde el último recambio de lámpara. Por estos motivos, en la última columna de la tabla se incluyen valores de $\mathrm{P}_{\mathrm{N}}$ estimados como iniciales, a los que se les ha considerado una depreciación de la instalación de un $20 \%$. Queda claro que no es posible asegurar que estos valores sean reales, pero sin duda constituyen una buena aproximación para estimar la eficacia inicial de la instalación.

\section{2.- Instalaciones con luminarias LED}

El rasgo distintivo de las luminarias LED para alumbrado público es la imposibilidad de diferenciar características funcionales de los LEDs o módulos LED de la propia luminaria. En efecto, el cuerpo o carcasa, que en luminarias tradicionales tiene como función principal brindar protección mecánica a la lámpara, aquí se comporta centralmente como disipador. De este modo, es imposible caracterizar al emisor luminoso aislado de su cuerpo o carcasa y por lo tanto, las características de emisión y potencia son únicas e indivisibles para cada módulo LED, su luminaria y equipo auxiliar (driver).

El flujo emitido por la luminaria, que para luminarias con lámpara de descarga caracterizábamos con (ver 9):

$$
\phi_{\text {Lum }}=\mathrm{LOR} \eta_{\mathrm{L}} \eta_{\mathrm{B}} \mathrm{P}_{\text {Lum }}
$$


Es ahora el resultado de uma medición fotométrica, al igual que la eficacia global de la luminaria LED:

$$
\eta_{\text {LUM }}=\phi_{\text {LUM }} / P_{\text {LUM }}
$$

La figura 85 muestra eficacias de luminarias LED estudiadas em el LAL. Los datos presentados se corresponden con luminarias comercializadas y también prototipos o pruebas previas que posiblemente no llegaron al mercado. Este comentario vale principalmente para los períodos 2011/2013. Las barras del diagrama no tienen una correspondencia cierta con una cantidad de luminarias estudiadas, solo muestran características de cada período. La media de pruebas ronda las 15/20 fotometrías anuales, exceptuando 2013, año en que la media fue superada justamente por la asistencia a empresas que ensayaron numerosos prototipos.

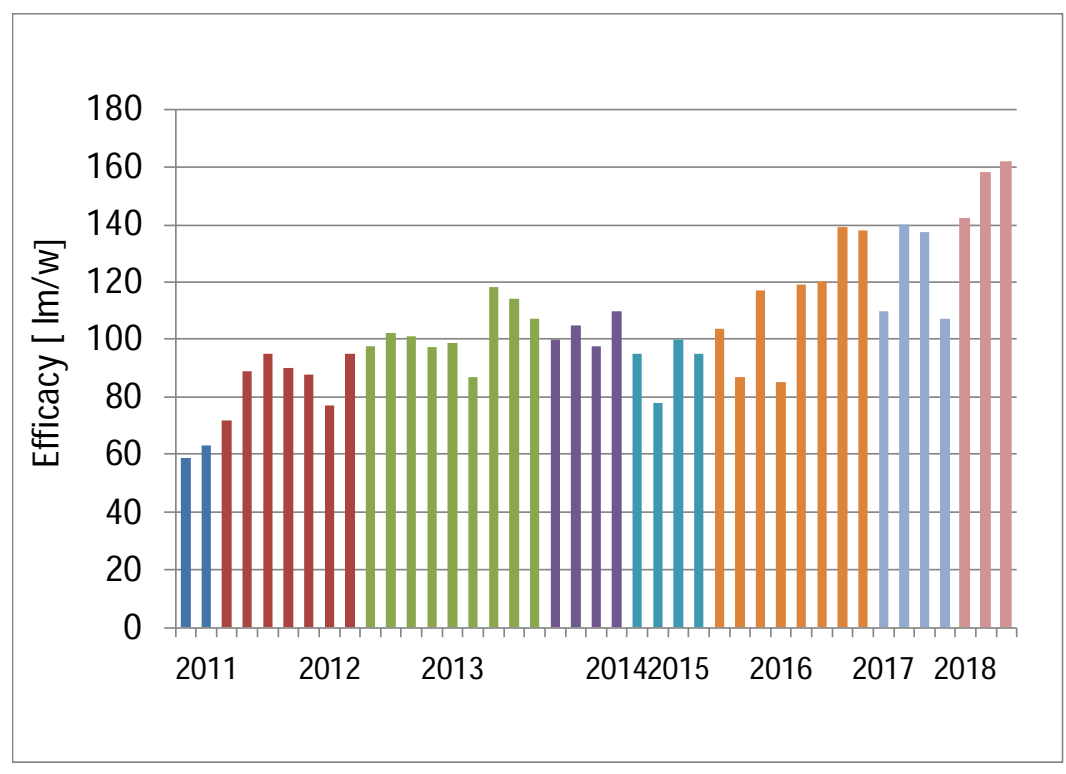

Figura 85. Eficacias de luminarias LED. ${ }^{14}$

Comparados con los valores indicados em la tabla 10, se observa que la eficacia de las luminarias LED supera claramente a las lámpras SAP a partir de 2015/16, siendo la diferencia actual (2018) del orden de un 30\%.

Finalmente, se presentan en la tabla 12 evaluaciones realizadas a instalaciones de autopistas reconvertidas a LED entre 2012 y 2014. En todos los casos, el pasaje a LED mantuvo los mismos parámetros geométricos (vanos, alturas de montaje, etc.) esto es, la reconversión solo incluyó recambio de luminarias. Vale aclarara que estas mismas instalaciones fueron con anterioridad evaluadas con lámparas convencionales y formaron parte de lo resumido en la tabla 11.

\begin{tabular}{|c|c|c|c|c|c|c|}
\hline Instalación & $\begin{array}{c}\text { Altura } \\
\text { de } \\
\text { montaje }\end{array}$ & $\begin{array}{c}\text { SxA } \\
{[\mathbf{m} 2]}\end{array}$ & $\begin{array}{c}\text { Potencia } \\
\text { de } \\
\text { lámpara }\end{array}$ & $\begin{array}{c}\text { Potencia } \\
\text { total }\end{array}$ & $\begin{array}{c}\mathbf{L m} \\
{[\mathbf{c d} / \mathbf{m} 2]}\end{array}$ & $\begin{array}{c}\mathbf{P}_{\mathrm{N}} \\
{[\mathbf{W} / \mathbf{c d}]}\end{array}$ \\
\hline
\end{tabular}

\footnotetext{
${ }^{14}$ Actualización de lo presentado en: Ixtaina, P., et al, “Argentinean Experience in...”, op. cit.
} 


\begin{tabular}{|c|c|c|c|c|c|c|}
\hline $\begin{array}{c}\text { Central, cuatro } \\
\text { luminarias por } \\
\text { columna }\end{array}$ & $16 \mathrm{~m}$ & 624 & $208 \mathrm{~W}$ & $416 \mathrm{~W}$ & 2,30 & 0,29 \\
\hline $\begin{array}{c}\text { Central, cuatro } \\
\text { luminarias por } \\
\text { columna }\end{array}$ & $15 \mathrm{~m}$ & 310 & $135 \mathrm{~W}$ & $270 \mathrm{~W}$ & 3,79 & 0,23 \\
\hline $\begin{array}{c}\text { Central, cuatro } \\
\text { luminarias por } \\
\text { columna }\end{array}$ & $16 \mathrm{~m}$ & 840 & $285 \mathrm{~W}$ & $570 \mathrm{~W}$ & 2,15 & 0,38 \\
\hline $\begin{array}{c}\text { Central, cuatro } \\
\text { luminarias por } \\
\text { columna }\end{array}$ & $16 \mathrm{~m}$ & 710 & $285 \mathrm{~W}$ & $570 \mathrm{~W}$ & 2,11 & \\
\hline
\end{tabular}

Tabla 12. Instalación reconvertidas a led..$^{15}$

\section{3.- Influencia del pavimento}

Con el objetivo de evaluar el efecto de las propiedades reflectivas de la calzada en el balance energético de la instalación, el coeficiente qoo (3) fue calulado para más de 300 mediciones simultáneas de luminancia e iluminancia relizadas por el LAL en un período de más de 10 años (1998-2012). Todas las mediciones formaron parte de los acuerdos LAL- OCCOVI y LAL-AUSA ya mencionados en el capítulo 4 y se efecturon bajo la normativa nacional IRAM. ${ }^{16}$

El conjunto de mediciones fue agrupado por secciones, tomando en cuenta los siguienes aspectos:

- Cada sección tenía el mismo tipo de asfalto y se computaron zonas con superficies homogéna ( $\sin$ bacheos o alteraciones).

- Dentro del período estudiado, la sección no tuvo repavimentaciones o cambos de compuesto.

- $\quad$ Cada sección mantuvo una geometría de instalación de alumbrado fija.

La figura 86 muestra los resultados de una sección típica. Los valores de luminancia e iluminancia del gráfico (Lav y Eav) son los valores promedio de cada sección analizada. La buena correlación entre Lav y Eav obtenida en cada caso permitió asignar un coeficiente qoo para cada una.

\footnotetext{
15 Tabla extraída de: Ixtaina, P., et al, "Argentinean Experience in...”, op. cit.
}

${ }^{16}$ Instituto Argentino de Racionalización de Materiales, 1995, op. cit. 


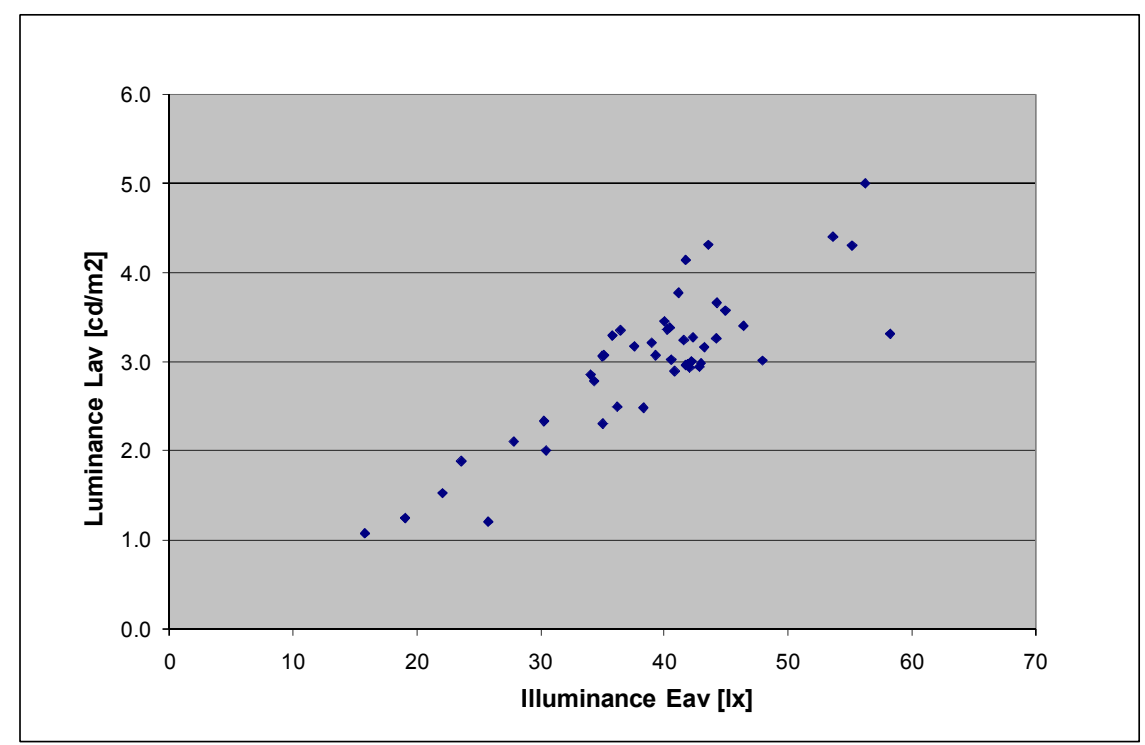

Figura 86. Luminancias e iluminancias típicos para una sección. ${ }^{17}$

Para estimar la dispersión obtenida para qoo, se calculó la incertidumbre estándar y expandida, siguiendo las pautas de ISO GUM. ${ }^{18}$ Se consideró una dispersión estadística para los valores calculados qoo (incertidumbre de tipo A) junto con el componente de incertidumbre debido a errores de medición (tipo B). Los resultados para la sección analizada previamente (y mostrada en la figura 4) se pueve ver en la tabla 13.

\begin{tabular}{|c|c|c|c|c|c|c|}
\hline $\begin{array}{l}\text { Source of } \\
\text { Uncertainty }\end{array}$ & Type & \multicolumn{2}{|c|}{ Uncertainty value } & $\begin{array}{l}\text { Sensitivity } \\
\text { coefficient }\end{array}$ & $\begin{array}{l}\text { Probability } \\
\text { Distribution }\end{array}$ & $\begin{array}{l}\text { Standard } \\
\text { uncertainty }\end{array}$ \\
\hline Repetibility & A & p. $\sigma / \sqrt{ } n$ & $\begin{array}{l}0,0043 \\
\mathrm{~cd} / \mathrm{m} 21 \mathrm{x}\end{array}$ & 1 & Normal & $\begin{array}{c}0,0043 \\
\mathrm{~cd} / \mathrm{m} 21 \mathrm{x}\end{array}$ \\
\hline $\begin{array}{c}\text { Eav } \\
\text { measurement }\end{array}$ & B & $\mathrm{E}_{\mathrm{Eav}} / \sqrt{3}$ & $1,12 \mathrm{~lx}$ & $\begin{array}{c}0,002 \\
\mathrm{Cd} / \mathrm{m} 21 \times 2\end{array}$ & Rectangular & $\begin{array}{c}0,0022 \\
\mathrm{~cd} / \mathrm{m} 21 \mathrm{x}\end{array}$ \\
\hline $\begin{array}{c}\text { Lav } \\
\text { measurement }\end{array}$ & B & $\mathrm{E}_{\text {Lav }} / \sqrt{3}$ & $\begin{array}{c}0,12 \\
\mathrm{~cd} / \mathrm{m} 2\end{array}$ & $0,0261 / 1 \mathrm{x}$ & Rectangular & $\begin{array}{l}0,0031 \\
\mathrm{~cd} / \mathrm{m} 21 \mathrm{x}\end{array}$ \\
\hline Qoo & Combined & - & - & - & Normal & $\begin{array}{l}0,0057 \\
\mathrm{~cd} / \mathrm{m} 21 \mathrm{x}\end{array}$ \\
\hline & & & & & $077 \pm 0.0$ & $\mathrm{~m} 21 \mathrm{x}$ \\
\hline
\end{tabular}

Notas:

$\sigma:$ Desviación estándar del qoo promedio

P: Coeficiente de Student para $95 \%$ de covertura

$\mathrm{N}$ :Grados de libertad

E: Error de medida

Tabla 13. Evaluación estadística. ${ }^{19}$

Es importante destacar que la repetibilidad (incertidumbre de tipo A) y la incertidumbre de medición combinada (tipo B) tienen un orden de magnitud similar. Esto permite

\footnotetext{
${ }^{17}$ Gráfico extraído de: Ixtaina, P., Vidal, P., "Road surface lightness...”, op. cit.

${ }^{18}$ International Organization for Standardization ISO, 1993, op. cit.

19 Tabla extraída de: Ixtaina, P., Vidal, P., "Road surface lightness...”, op. cit.
} 
considerar a qoo como una buena aproximación a Qo. Es una relación que se mantuvo para todas las secciones estudiadas, como se muestra en la tabla 14.

\begin{tabular}{|c|c|c|c|}
\hline \multicolumn{2}{|c|}{ Motorway } & \multicolumn{2}{c|}{ q00 [cd/m2Ix] } \\
\hline M1 & La Plata BA City s1 & $0.077 \pm 0.006$ \\
\hline M2 & La Plata BA City s2 & $0.085 \quad \pm \quad 0.006$ \\
\hline M3 & BA - South Access & $0.079 \quad \pm \quad 0.008$ \\
\hline M4 & BA - West Access & $0.082 \quad \pm \quad 0.009$ \\
\hline M5 & Panamericana Motorway & $0.107 \quad \pm \quad 0.012$ \\
\hline M6 & BA - Tigre Section & $0.101 \quad \pm \quad 0.010$ \\
\hline M7 & Ezeiza Cañuelas Access & $0.061 \quad \pm \quad 0.006$ \\
\hline
\end{tabular}

Tabla 14. Resultado del análisis para todas las secciones estudiadas.

\subsection{Eficiencia energética de la instalación.}

El coeficiente de luminancia media (grado de "claridad"), evaluado con qoo y la eficiencia energética están directamente relacionados. Si se supone un factor de uso $\mu \mathrm{u}$ fijo, la densidad de la potencia normalizada $\mathrm{P}_{\mathrm{N}}$ puede expresarse a partir de (2) y (12) como:

$$
P_{N}=\frac{P_{D}}{L_{a v}}=\frac{P_{D}}{\text { Eav qoo }}=\frac{\text { Cte }}{\text { qoo }}
$$

En esta condición, el aumento de qoo es seguido por un cambio de la potencia normalizada en la misma proporción pero en dirección inversa.

Ahora bien, si en la etapa de diseño se usa, como ya se vio, el presupuesto erróneo de representar la superficie de la calzada por un pavimento estándar R3, su coeficiente de luminancia queda fijo (Qo de la tabla-r R3 es 0,07 cd/m21x). Con esto, los cambios en la eficiencia energética debido a la luminosidad real de la superficie de la carretera no se consideran en la etapa del proyecto. La Figura 87 muestra las diferencias entre los qoo obtenidos en nuestro estudio y el coeficiente de luminancia promedio para la superficie estándar R3. 


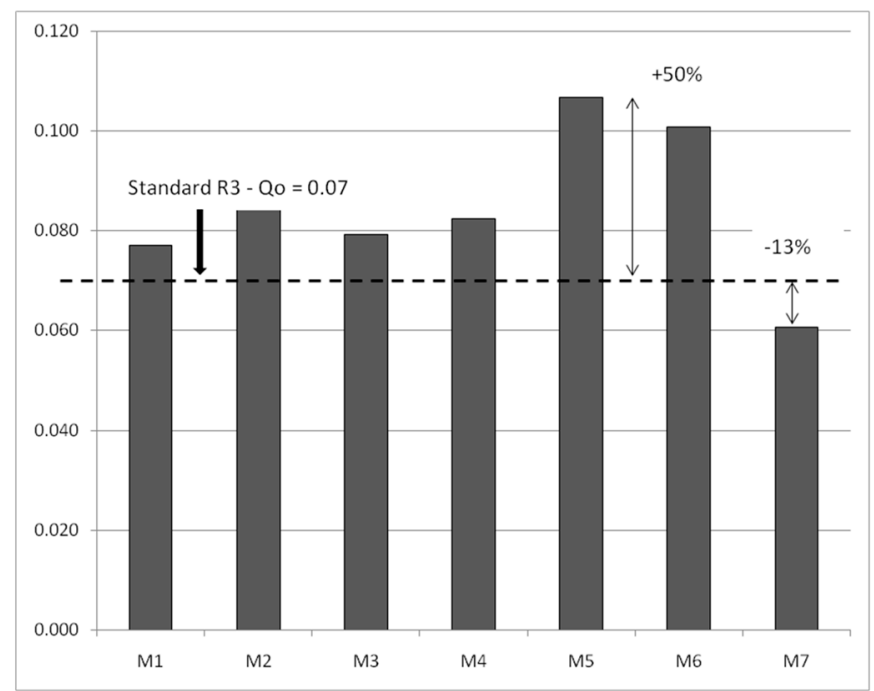

Figura 87. Coefcientes qoo obtenidos comparados con el Qo del estándar R3.

La figura 87 muestra las diferencias en "claridades" que son desechadas con las prácticas habituales. Por ejemplo, la sección de autopista identificada como M5 posee un 50\% más en qoo respecto al Qo del R3, o sea, el flujo luminoso necesario para llegar al nivel de luminancia media de diseño (Lav) podría reducirse proporcionalmente, con el consiguiente aumento en la eficiencia. Por supuesto, la situación opuesta también puede presentarse: una superficie "oscura" (calzada de la sección M7) daría un parámetro de Lav deficiente a la par que reduce la eficiencia del sistema.

\section{4.- Algunas conclusiones}

Este capítuo aporta un nuevo elemento para resaltar la importancia de conocer las características reflectivas reales de la superficie de la carretera de la instalación, concepto generalmente ignorado en la mayoría de los diseños. En este caso, se hizo referencia a un solo aspecto del fenómeno: la diferencia de "luminosidad" entre las superficies de las carreteras consideradas en el diseño y en las reales. Esta discrepancia fue uno de los factores que llevaron a sobre dimensionar la mayoría de las instalciones argentinas en la década del "90. Es un aspecto que puede calificarse como "energético", se suma a lo demostrado en capítulos previos y tiene como eje las "fallas" en el conocimiento de la "forma" de la matriz de reflexión; este es diferente de la del estándar y conduce a pérdidas o inexactitudes en las uniformidades en luminancia de las intalaciones evaluadas $[10,11] .^{20}$

\footnotetext{
${ }^{20}$ Ixtaina, P. et al, "Effects of led lighting...", op. cit.; Ixtaina, P., Vidal, P., "Road surface lightness...", op. cit.
} 
Capítulo 7 - Reflexión en calzadas y espectro de la fuente luminosa 
En los capítulos precedentes se profundizó en las propiedades reflectivas de las calzadas, con perspectiva local, es decir, tomando en cuenta las características propias de la zona metropolitana de Buenos Aires, Argentina. Los estudios de campo junto con las experiencias realizadas permitieron corroborar la importancia de la correcta caracterización, su influencia en el consumo de energía y la necesidad de contar con datos de reflexión cuando se alcanzan las condiciones de estabilidad de las carpetas asfálticas. Lo anterior, como ya se dijo, verifica la hipótesis de partida: no es válida la extrapolación de datos de otras regiones y con ello se remarca el error de aplicar la Técnica de Luminancia sobre la base de pavimentos estándares europeos.

En este marco, este último capítulo aborda el vínculo entre reflexión en calzada y espectro de la fuente luminosa. Comienza describiendo algunos antecedentes, entre ellos estudios realizados en regiones (países nórdicos) que utilizan compuestos "aclarantes" en las mezclas asfálticas. Asimismo, y como rasgo central de la bibliografía encontrada, los espectros comparados hacen referencia a lámparas de descarga, típicas del alumbrado público y vial hasta los últimos años. Luego, se focalizará en los espectros LED, fuentes luminosas que claramente se imponen como exclusivas para el futuro cercano.

\section{1.- Coeficiente de lumínica. Evaluación del grado de claridad del pavimento}

Tal como se explicó antes, en el marco de la Técnica de Luminancia, las propiedades de reflexión de la superficie del camino se caracterizan por medio del coeficiente de luminancia "q", factor de proporcionalidad, para cada punto de la calzada, entre su iluminancia y la luminancia reflejada en la dirección de observación. Asimismo, se explicó que el factor de proporcionalidad o escala entre distintas tablas-r se obtiene a partir de la integral del coeficiente de luminancia " $q$ " sobre un ángulo sólido que subtienda a un elemento de calzada. El coeficiente resultante, coeficiente de luminancia medio Qo, evalúa de alguna manera el grado de "claridad" de la superficie del camino.

Como se indicó en el capítulo 6, el Qo del pavimento se vincula directamente con la eficiencia energética de la instalación. Una calzada "más clara" (y con Qo mayores) generará un incremento de las luminancias en dirección al observador para una misma distribución de iluminancias. Resulta entonces de interés estudiar si este "grado de claridad" puede cambiar con el espectro de la luz usada en el sistema de iluminación, así como los posibles efectos de "selectividad espectral" que influencien la reflexión de la luz blanca del led.

\section{1.- El coeficiente qoo}

Si se cuenta con una cantidad suficiente de evaluaciones simultáneas de luminancias e iluminancias puntuales en varias secciones de una vía de tránsito, es posible utilizar el factor qoo -relación entre luminancia media e iluminancia media-, como una aproximación empírica al grado de claridad de la calzada:

$$
\mathrm{q}_{00}=\frac{\mathrm{Lm}}{\mathrm{Em}}
$$

Aunque no existe ninguna relación teórica entre las definiciones de Qo y qoo, la baja dispersión obtenida en el análisis de un número importante de evaluaciones de luminancia 
e iluminancia, permite suponer un buen desempeño de este coeficiente como indicador del grado de claridad de la calzada, ${ }^{1}$ salvando la casi imposibilidad práctica de medir Qo sobre una calzada.

El un estudio previo, ${ }^{2}$ se verificaron diferencias de importancia en el "grado de claridad" de las calzadas de las autopistas de acceso y urbanas de la ciudad de Buenos Aires. Allí se tuvieron como base más de 300 evaluaciones simultáneas de luminancia e iluminancia, efectuadas en autopistas de la mencionada zona, para el período 1998-2012. Todas las mediciones se llevaron a cabo en instalaciones con lámparas de vapor de sodio y siguieron los procedimientos de medición estandarizados por la recomendación argentina. ${ }^{3}$

\section{2.- Grado de claridad bajo iluminación led}

Dicho lo anterior, se propone verificar si existe algún cambio en el coeficiente de luminancia medio atribuible a la luz blanca del led, es decir, si es posible hallar algún tipo de selectividad espectral en la reflexión sobre la calzada.

\section{1- Antecedentes}

Ekrias $^{4}$ estudió la reflexión espectral de once tipos de compuestos asfálticos de Finlandia, combinando muestras de superficies "naturales" y con agregados de pigmentos colorantes o clarificadores. Sus mediciones se basaron en muestras circulares de $100 \mathrm{~mm}$ de diámetro, ángulos de incidencia $\beta=20^{\circ}$ y elevación $\gamma=55^{\circ}$. El ángulo de observación $\alpha$ fue de $35^{\circ}$, mayor al estándar CIE de $1^{\circ}$. La figura 88 presenta algunas de las muestras empleadas en dicha investigación que permiten visualizar un tamaño de piedra importante, con una proporción de compuesto asfáltico aglutinante notoriamente menor a la de los pavimentos en uso local (comparar con las muestras presentadas en el capítulo 4). Asimismo, algunas de las muestras manifiestan una tonalidad rojiza, fruto posiblemente de los agregados colorantes.

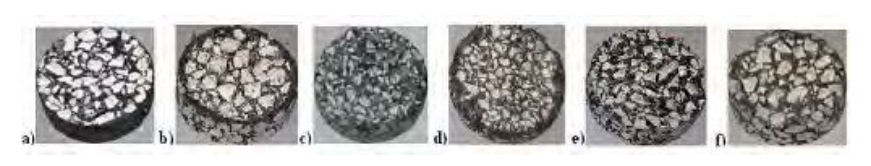

Figura 88. Muestras de pavimentos de estudio de Ekrias. ${ }^{5}$

La tonalidad mencionada se pone de manifiesto en los espectros obtenidos por Ekrias,

\footnotetext{
${ }^{1}$ Ixtaina, P., Vidal, P., "Road surface lightness and energy efficiency in road lighting", Proceedings of the 12th European Lighting Conference “LUX EUROPA 2013”, Cracovia (Polonia), 2013; Ixtaina, P., Armas, A., Bannert, B., "Use effects on the reflection of macro textured surfaces", Journal of Applied Engineering Sciences, 6(19), 2016a, pp. 51-56.

${ }^{2}$ Ixtaina, P., Vidal, P., "Road surface lightness...", op. cit.

${ }^{3}$ Instituto Argentino de Racionalización de Materiales, IRAM AADL J 2022-2, Alumbrado Público, Vias de Tránsito - Clasificación y Niveles de Iluminación, Buenos Aires, 1995.

${ }^{4}$ Ekrias, A., Ylinen, A., Eloholma, M., Halonen, L., "Effects of Pavement Lightness and Colour on Road Lighting Performance", CIE International Symposium on road surface photometric characteristics: Measurement Systems and Results, Italia, 2008.

${ }^{5}$ Imagen extraída de: Ekrias, A., Ylinen, A., Eloholma, M., Halonen, L., “Effects of...”, op. cit.
} 
que evidencian un ligero incremento en su reflectancia hacia la zona roja del espectro.

Por otra parte, los estudios de Adrian ${ }^{6}$ muestran resultados similares. Allí, las muestras recolectadas fueron concretos asfálticos y hormigones, sin especificación del uso de agregados colorantes. La figura 89 muestra un crecimiento en la reflectancia para longitudes de onda crecientes, similar al encontrado en Ekrias.

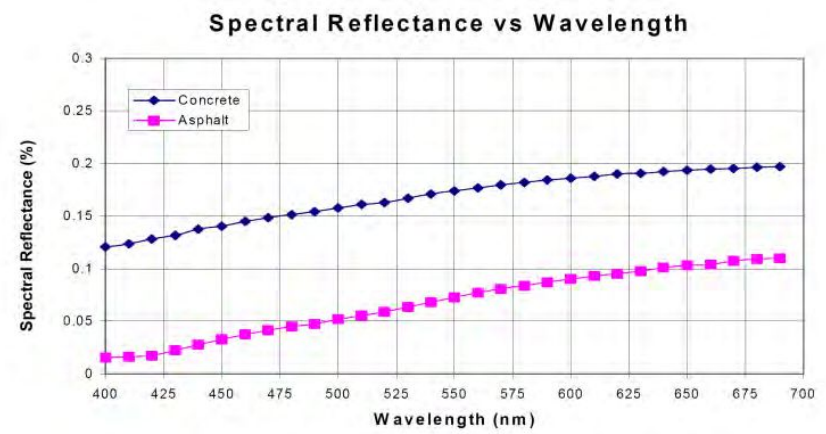

Figura 89. Resultados de los estudios espectrales de Adrian. ${ }^{7}$

Estudios americanos ${ }^{8}$ exhiben un incremento en la reflectancia hacia el rojo mayor, que resulta más notorio en superficies desgastadas por varios años de uso (figura 90). Es interesante notar la coincidencia entre investigaciones de lugares distantes (Estados Unidos, Europa), pese a la alta influencia regional en la composición del pavimento y al uso -o no uso- de colorantes agregados.

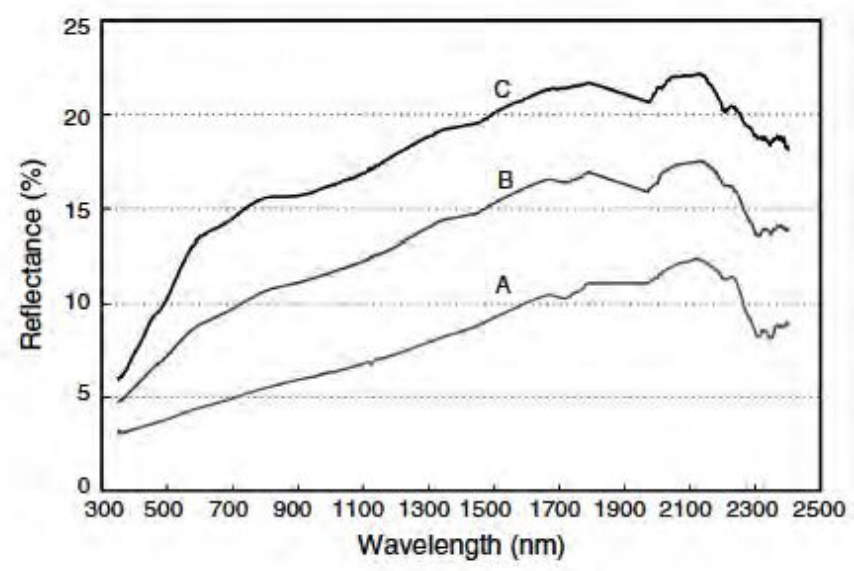

Figura 90. Estudios de Herold (EE.UU.). Pavimento C, con más de 10 años de uso. ${ }^{9}$

\section{2- qoo con Iluminación Led}

\footnotetext{
${ }^{6}$ Adrian, W., Jobanputra, R., "Influence of Pavement Reflectance on Lighting for Parking Lots", Portland and Cement Association, PCA R\&D Serial No 2458, 2005.

${ }^{7}$ Gráfico extraído de: Adrian, W., Jobanputra, R., "Influence of Pavement...”, op. cit.

${ }^{8}$ Herold, M. Remote Sensing of Impervious Surfaces, Ch. 12 - Spectral Characteristics of Asphalt Road Surfaces, Wageningen University, 2007.

${ }^{9}$ Imagen extraída de: Herold, M. Remote Sensing of..., op. cit.
} 
Siguiendo el modelo de experiencia descripta en un trabajo previo, ${ }^{10}$ se analizaron mediciones simultáneas de luminancia e iluminancia en instalaciones de autopistas con iluminación led. Estas evaluaciones se realizaron en caminos de la Red de Accesos a Buenos Aires y en otros de la ciudad (Red AUSA), entre 2013 y 2015. En todos los casos se siguió el procedimiento estandarizado en el país ${ }^{11}$ y las mediciones formaron parte de las transferencias tecnológicas del LAL. ${ }^{12}$

Los casos evaluados fueron instalaciones reconvertidas a led (6 áreas de medición, en la autopista 25 de Mayo y AU6, Perito Moreno) y pruebas de artefactos leds (Autopista La Plata-Buenos Aires y Ramal Campana de la Autopista Acceso Norte). Estas últimas se basaron en la medición de tramos "testigos", conformados por el reemplazo de -al menoslas luminarias de 4 columnas consecutivas de un sector de la autopista. El área de relevamiento se ubicó en el vano central ( 5 casos). Todas las pruebas consideradas tenían su correlato con artefactos que empleaban lámparas de descarga (Sodio Alta Presión, SAP) y se encontraban contabilizadas en el estudio. ${ }^{13}$ Asimismo, se procuró incluir solo aquellas áreas de evaluación que no tuvieran cambios extremos en la carpeta de rodamiento. La tabla 15 resume los casos analizados:

\begin{tabular}{|c|c|c|c|}
\hline Autopista & Áreas evaluadas & $\begin{array}{c}\text { Qoo (medio) } \\
{\left[\mathrm{cd} / \mathrm{m}^{2} \mid \mathrm{x}\right]}\end{array}$ & $\begin{array}{c}\text { Incertidumbre } \\
\text { estándar } \\
{\left[\mathrm{cd} / \mathrm{m}^{2} \mathrm{l}\right]}\end{array}$ \\
\hline $\begin{array}{c}\text { La Plata Bs As } \\
\text { Tramo1 }\end{array}$ & 8 & 0,0860 & 0,006 \\
\hline $\begin{array}{c}\text { La Plata Bs As } \\
\text { Tramo2 }\end{array}$ & 4 & 0,1080 & 0,010 \\
\hline Urbanas Bs As & 6 & 0,0828 & 0,005 \\
\hline Panamericana & 2 & 0,0640 & $-\ldots$ \\
\hline
\end{tabular}

Tabla 15. Instalaciones leds evaluadas.

En línea con la Organización Internacional de Estandarización (ISO) ${ }^{14}$ la incertidumbre estándar, considerando solo la parte atribuible a la componente tipo A, fue evaluada como:

$$
\mu=\frac{\sigma n-1}{\sqrt{n}}
$$

Pese a que los casos considerados fueron acotados, la incertidumbre resultó del mismo orden de magnitud que en el comentado análisis, ${ }^{15}$ por lo que las estimaciones de qoo para iluminación led pueden considerarse representativas de cada tipo de pavimento. Una excepción a este comentario es el último caso, sobre el que se hicieron dos mediciones, y se incluye solo a título ilustrativo.

\footnotetext{
${ }^{10}$ Ixtaina, P., Vidal, P., "Road surface lightness...”, op. cit.

${ }^{11}$ Instituto Argentino de Racionalización de Materiales, 1995, op. cit.

${ }^{12}$ Ver por ejemplo: Ixtaina, P., Informe Cientifico del Investigador 2013/14, Repositorio digital CIC [en línea] URL: http://digital.cic.gba.gob.ar/handle/11746/1869 [Consulta: 3 de abril de 2019]; Ixtaina, P., Informe Científico del Investigador 2015/16, Repositorio digital CIC, [en línea] URL: http://digital.cic.gba.gob.ar/handle/11746/6450 [Consulta: 3 de abril de 2019].

${ }^{13}$ Ixtaina, P., Vidal, P., "Road surface lightness...", op. cit.

14 International Organization for Standardization ISO, "Guide to the expression of uncertainty in measurement", Suiza, 1993.

${ }^{15}$ Ixtaina, P., Vidal, P., "Road surface lightness...”, op. cit.
} 


\section{3- Resultados}

La figura 91 muestra las relaciones luminancia media - iluminancia media, para cada una de las secciones estudiadas.

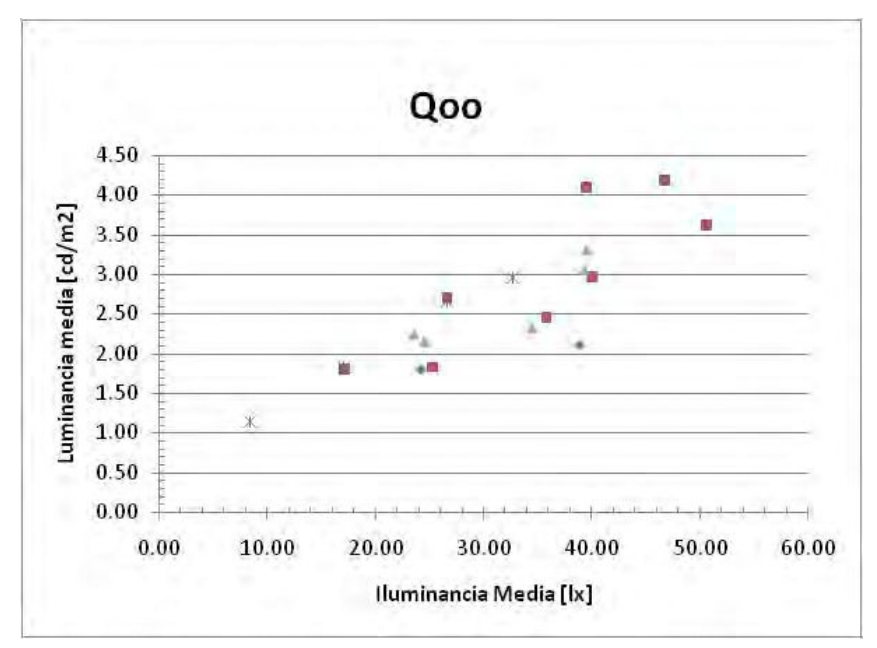

Figura 91. Relación luminancia - iluminancia para los tramos leds estudiados.

En la figura 92 se comparan, para cada zona, los valores de qoo obtenidos con iluminación tradicional (sodio alta presión) y las nuevas luminarias leds.

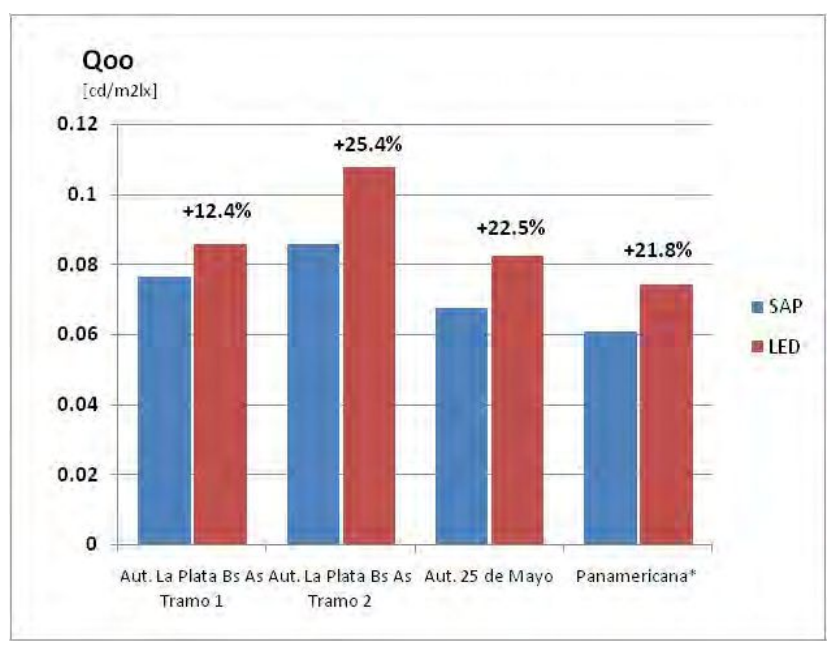

Figura 92. Comparación del qoo obtenido para instalaciones con sodio alta presión y leds.

Los resultados obtenidos indican un aparente incremento en el "grado de claridad" de cada superficie de calzada para el espectro de la luz led. En un primer análisis, este efecto es coincidente con los estudios previos, al menos en el hecho cualitativo de influencia del espectro de la fuente en la reflexión media del pavimento; en otras palabras, la reflexión no puede considerarse acromática. Sin embargo, no es claro el vínculo entre el incremento de qoo para el espectro led, con la tonalidad "rojiza" de los pavimentos estudiados por Ekrias, Adrian y Herold. Esta tendencia, combinada con el espectro predominantemente azul del led, induce a pensar en un resultado opuesto al encontrado en nuestro estudio. Coherente 
con este último razonamiento, en el texto de Ekrias, ${ }^{16}$ se menciona una mejora en Qo para superficies iluminadas con lámpara de sodio, con respecto al mismo pavimento bajo luz blanca (mercurio alta presión).

Por otra parte, no puede afirmarse que los pavimentos europeos o estadounidenses de las investigaciones citadas sean comparables a los actualmente en uso en Argentina, los cuales fueron objeto de los análisis propios. La figura 93 muestra probetas de tales calzadas, del tipo "drenante", en las que se observa una granulometría y color distintos de los presentados en la figura 88. Está claro que la comparación entre fotografías tiene solo un valor descriptivo, pero indica para las superficies locales una densidad (al menos superficial) mayor de aglutinante con piedras de menor tamaño. Asimismo, las imágenes no presentan evidencias de tonalidades rojizas.

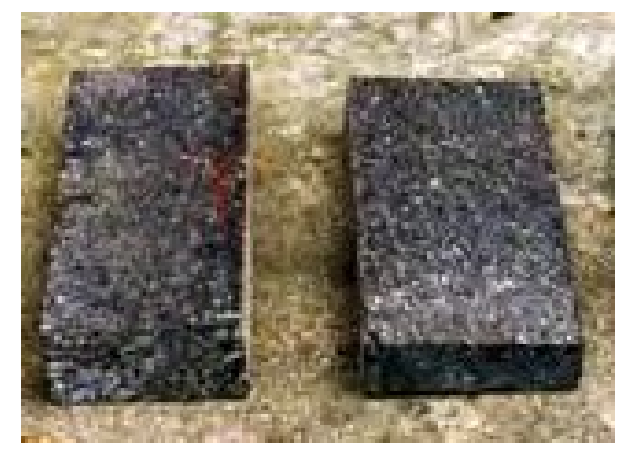

Figura 93. Muestras (probetas) de superficies drenantes, similares a las calzadas de las autopistas estudiadas (foto del autor).

\section{Estudio espectral}

\section{1- Esquema de medición}

Se trabajó sobre una probeta -similar a las presentes en la figura 93-de dimensiones estandarizadas para evaluación de muestras, ${ }^{17}$ montada en el equipo de medición de la tabla$\mathrm{r}$ del LAL-CIC (Reflectómetro de muestras, ver capítulo 4). La fuente luminosa se ubicó en $\beta=0^{\circ}$, utilizando tres ángulos de incidencia vertical: $\gamma=0^{\circ}, 15^{\circ}$ y $30^{\circ}$.

El espectro reflejado por la muestra se registró con un espectrómetro Avantes Starline, AvaSpec $2048,{ }^{18}$ con ángulo de observación estándar CIE, $\alpha=1^{\circ}$.

La figura 94 muestra un esquema del sistema de medición, las figuras 95 y 96, aspectos de la experiencia.

\footnotetext{
${ }^{16}$ Ekrias, A., Ylinen, A., Eloholma, M., Halonen, L., "Effects of...”, op. cit.

${ }^{17} \mathrm{CIE}$, Calculation and measurement of luminance and illuminance in road lighting, Publicación 30-2 TC 4.6, Viena, 1982.

18 "StarLine", Avantes. Enlightening Spectroscopy, [en línea] URL: http://www.avantes.com/products/spectrometers/starline [Consulta: 3 de abril de 2019].
} 


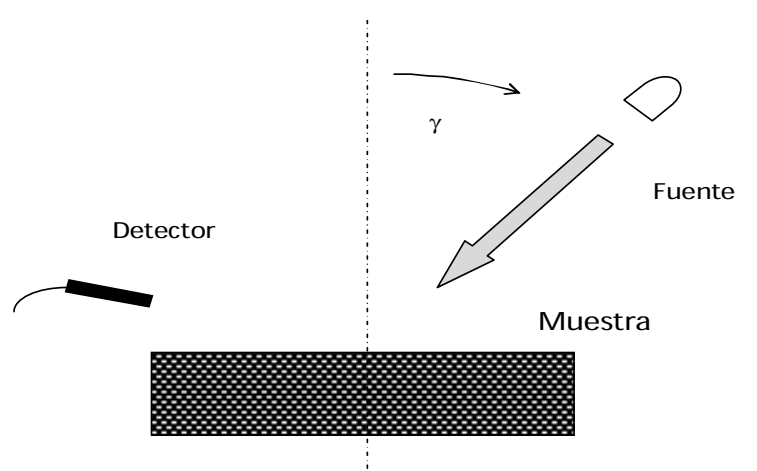

Figura 94. Esquema del sistema de medición.

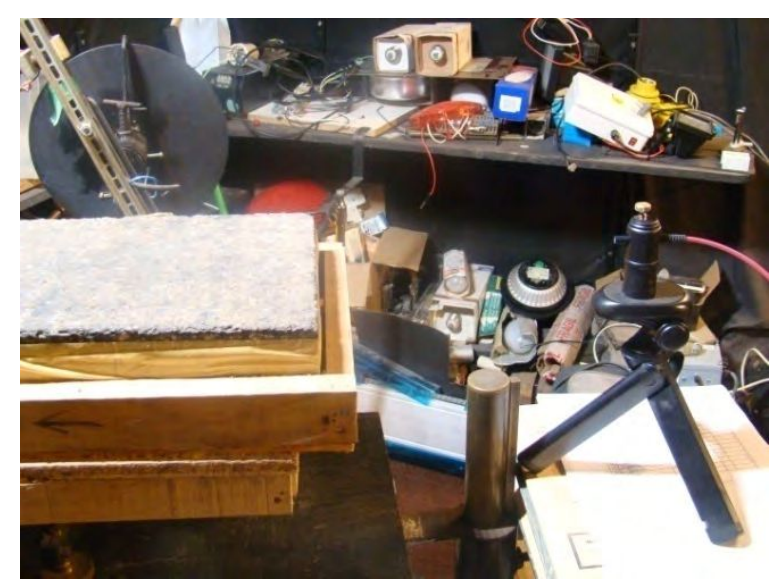

Figura 95. Aspectos de la experiencia. Detector (foto del autor).

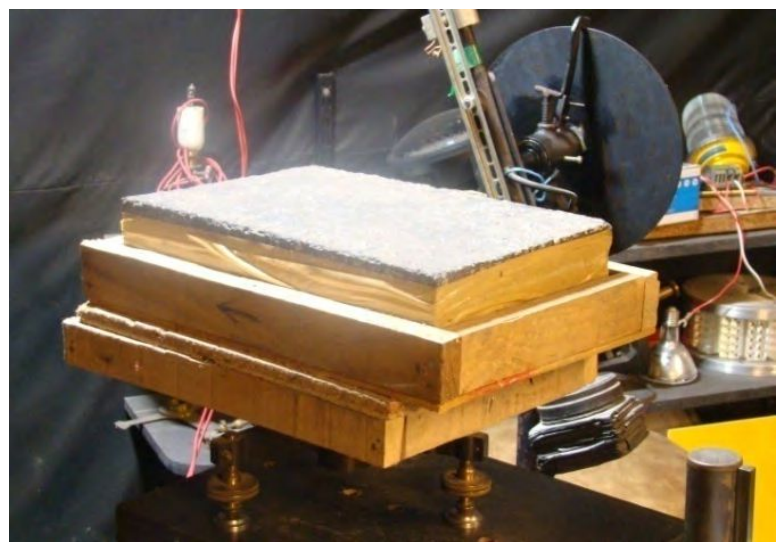

Figura 96. Aspectos de la experiencia. Muestra. (foto del autor).

En este proceso, se compararon dos fuentes luminosas. Por un lado, una lámpara de sodio alta presión (SAP), del tipo tubular clara, cuyo espectro se muestra en la figura 97. El espectro mostrado corresponde a una medición directa con el espectrómetro ya mencionado, enfocado en dirección normal al centro del "quemador", tubo de descarga de la lámpara. 


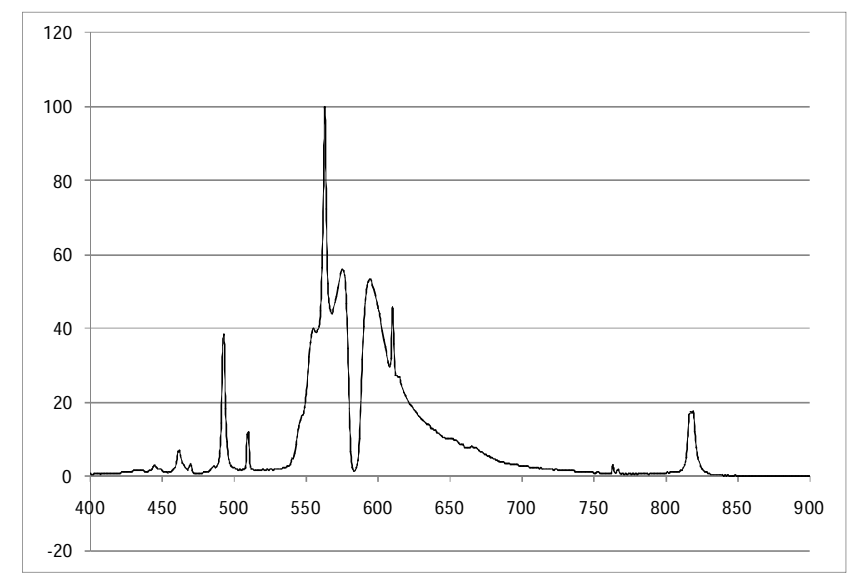

Figura 97. Espectro de la lámpara SAP (Medición con espectrómetro Avantes ${ }^{19}$ ).

En segundo lugar, como fuente led se empleó una placa con componentes SMD (LEDs montaje superficial, Surface Mounted Devices), sin lente refractora, y de características cromáticas $\mathrm{x}=0,362, \mathrm{y}=0,366, \mathrm{CCT}=4500 \mathrm{~K}$. La placa es una de las típicas usadas para la construcción de luminarias para alumbrado vial. Su espectro se representa en la figura 98. En forma similar a lo indicado, la medición se efectuó enfocando con el detector el haz central de la placa.

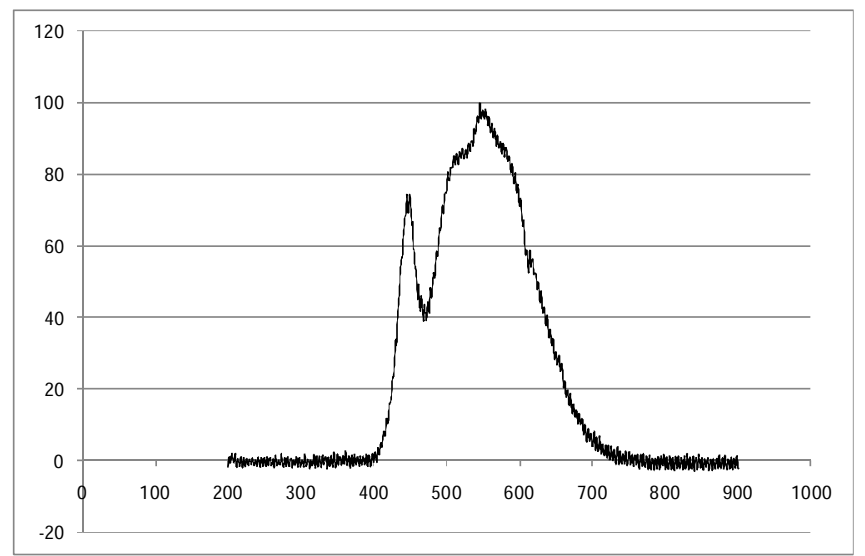

Figura 98. Espectro de la fuente led (medición con espectrómetro Avantes ${ }^{20}$ ).

\section{2- Resultados}

La comparación de los espectros directos y reflejados se efectuó a partir de re-escalarlos a valores porcentuales de sus respectivos máximos. La superposición de ambas curvas debería indicar (en el caso de no coincidencia) las zonas con diferencias en absorción espectral. La figura 99 compara los espectros para lámpara SAP, con incidencia $\gamma$ de $30^{\circ} \mathrm{y}$ es representativa de $\gamma 0^{\circ}$ y $15^{\circ}$. Se enfatizó en la zona del espectro con mayores diferencias, destacándose la región 560-580 $\mathrm{nm}$ y 590-630 nm, que presentan una absorción mayor que en el resto del espectro.

\footnotetext{
19 "StarLine", op. cit.

20 "StarLine", op. cit.
} 


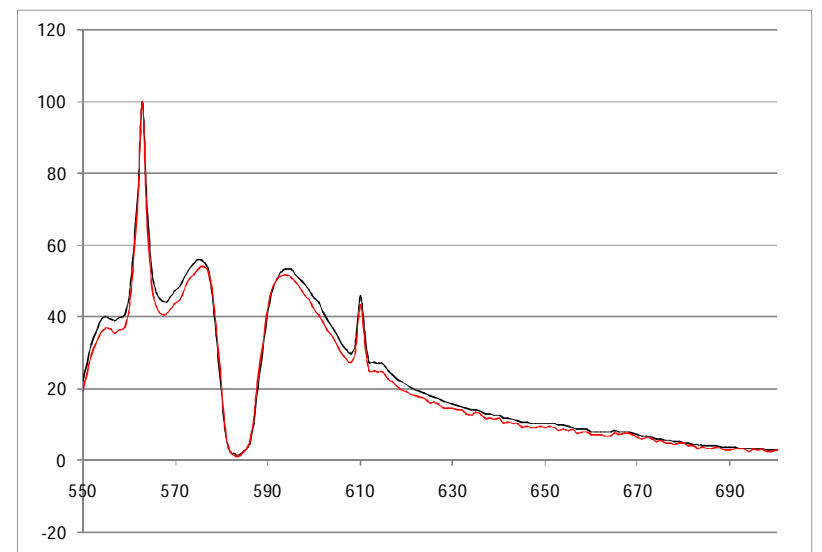

Figura 99. Espectro directo y reflejado para SAP.

En las zonas mencionadas, el cociente de ambas curvas (que debería centrarse en 1, por el re escalado), tiene tendencia a situarse en las inmediaciones de 0,9 , lo que indicaría una absorción un 10\% mayor en esta parte del espectro (figura 100).

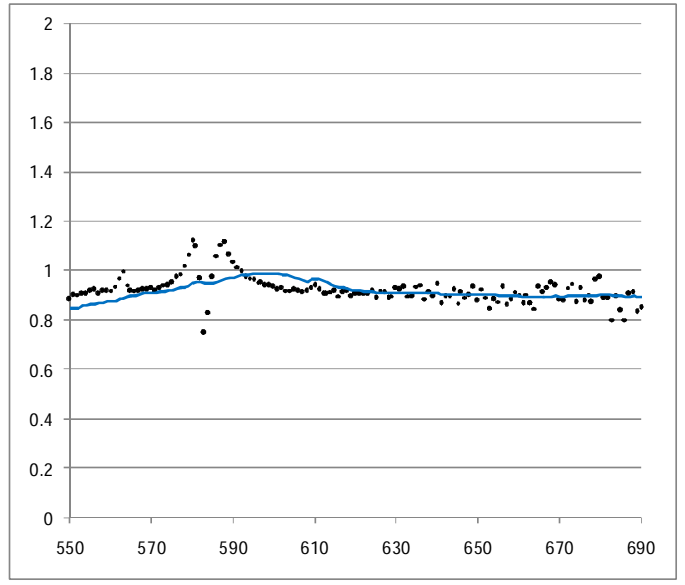

Figura 100. Relación espectro reflejado/directo.

La figura 101 presenta superpuestos, los espectros directos y reflejados para la fuente led. Exceptuando una pequeña diferencia en la región $450-500 \mathrm{~nm}$ y en torno a $650 \mathrm{~nm}$, ambas curvas parecen coincidentes, mostrando discrepancias menores que para el caso del sodio. La figura 102, que exhibe la relación espectro reflejado a directo, detalla este fenómeno.

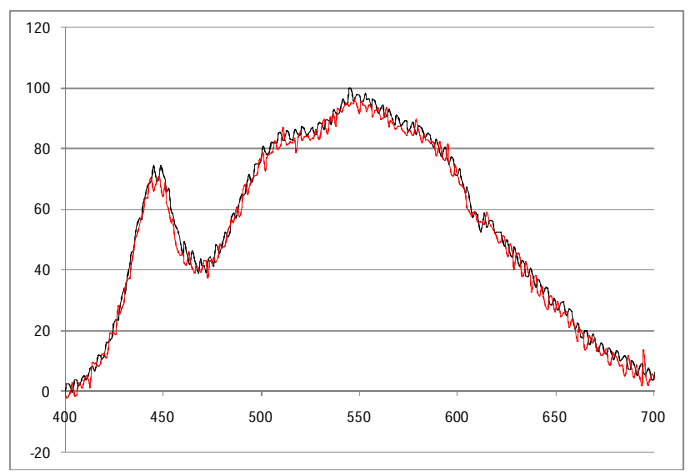


Figura 101. Espectro directo y reflejado para led.

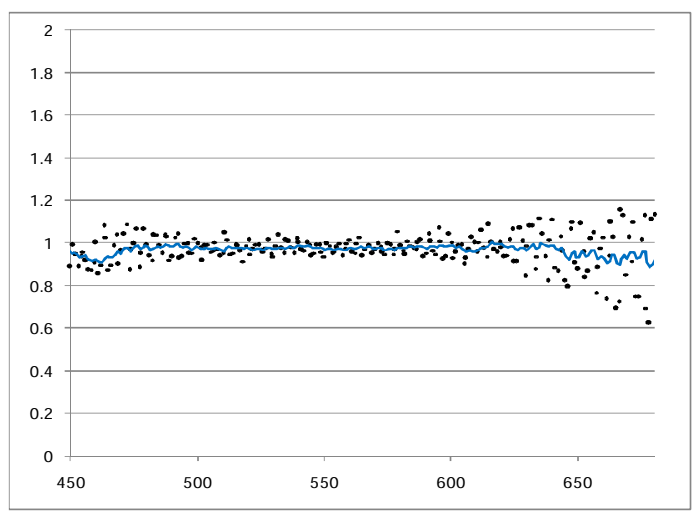

Figura 102. Relación espectro reflejado/directo.

En la zona visible del espectro, la figura 102 muestra una línea de tendencia muy cercana a 1 .

Para valorar el efecto "fotópico" de estas diferencias y poder cuantificar con un número único, representativo de la reflexión media en la zona visible (valor admitido solo para las condiciones de medición: $\alpha=1^{\circ}, \beta=0^{\circ}$ y $\delta=30^{\circ}$ ), se calcularon los factores F1 y F2 proporcionales a la emisión y reflexión fotópica y definidos como (ver capítulo 2):

$$
\begin{aligned}
& \mathrm{F} 1=\int \operatorname{Gdir}(\lambda) \mathrm{V}(\lambda) \mathrm{d} \lambda \\
& \mathrm{F} 2=\int \operatorname{Gref}(\lambda) \mathrm{V}(\lambda) \mathrm{d} \lambda
\end{aligned}
$$

En (3) y (4) $V(\lambda)$ es la curva de sensibilidad espectral normalizada del ojo ${ }^{21}$ y $G(\lambda)$ son los espectros medidos, "dir" directo y "ref" reflejado por la muestra en las condiciones ya mencionadas. La figura 103 muestra a modo de ejemplo, $\operatorname{Gref}(\lambda), \mathrm{V}(\lambda)$ y el producto, para el caso de lámpara de sodio.

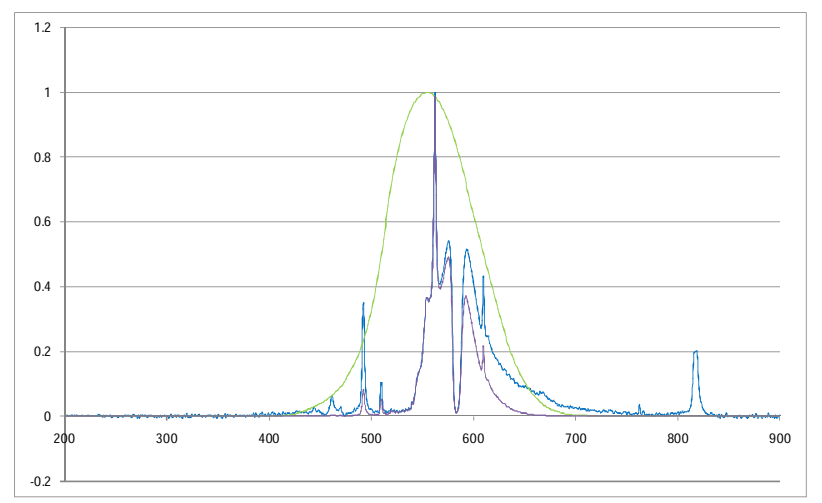

Figura 103. Espectro SAP reflejado y curva $V(\lambda)$.

La tabla 16 resume los resultados de los cálculos realizados. Se identifica una diferencia

21 CIE, Appendix 7: Photopic spectral luminous efficiency function, [en línea] URL: https://onlinelibrary.wiley.com/doi/pdf/10.1002/0470024275.app7 [Consulta: 3 de abril de 2019]. 
a favor del reflejo led ("ganancia") cercana al 4\% para el pavimento estudiado, de acuerdo con las condiciones de observación e iluminación ya estipuladas.

\begin{tabular}{|c|c|c|c|}
\hline Espectro & $\mathbf{F}=\int \mathbf{G}(\boldsymbol{\lambda}) \mathbf{V}(\boldsymbol{\lambda}) \mathbf{d} \boldsymbol{\lambda}$ & $\begin{array}{c}\text { Diferencia relativa } \\
(\mathrm{F} 2-\mathrm{F} 1 / \mathrm{F} 1)\end{array}$ & $\begin{array}{c}\text { Ganancia } \\
\text { Reflejo LED a } \\
\text { Sodio }\end{array}$ \\
\hline Sodio Directo & 25,00 & $-5,92 \%$ & \\
\hline Sodio reflejado & 23,52 & $3,8 \%$ \\
\hline LED directo & 85,30 & $-2,12 \%$ & \\
\hline LED reflejado & 83,49 & & \\
\hline
\end{tabular}

Tabla 16. Resultados comparación fotópica.

\section{4.- Conclusiones}

Este capítulo se centró en demostrar que la reflexión en la calzada depende del espectro de la fuente luminosa y que esta dependencia conlleva valores que deben tenerse en cuenta en el diseño de la instalación en virtud de su vínculo con la eficiencia energética y el uso de LEDs. El estudio explicitado aquí permite afirmar que el espectro led aumenta la reflexión y el efecto es mayor cuanto más frío es el led, existiendo una notoria selectividad hacia el azul para las calzadas analizadas. Asimismo, un abordaje más reciente, que empleó otro sistema experimental y nuevos espectros leds, ${ }^{22}$ arrojó resultados similares.

Cabe destacar que los resultados encontrados son coincidentes con los de investigaciones realizadas en Europa y Estados Unidos, si se tiene en cuenta la existencia de una sutil dependencia del reflejo en calzadas con el espectro de la luz incidente. Esto implicó, en tales pruebas, a una leve coloración hacia el verde-rojo, que surge en todos los antecedentes a pesar de las diferentes técnicas de análisis empleadas y del tipo y composición de los asfaltos observados. En esta Tesis, sin embargo, el incremento reflectivo fue más notorio para la región verde- azul del espectro.

El estudio con calzadas reales -en uso en la zona metropolitana de Buenos Airespermitió correlacionar esta "selectividad espectral" con un incremento (ganancia) en el grado de claridad cuando se ilumina con leds, frente al espectro del sodio alta presión. Las mejoras de qoo encontradas en estas autopistas resultaron del orden de un $20 \%$ promedio, mientras que, en el estudio espectral sobre una muestra, el incremento pudo estimarse en alrededor de un 4\%. Si bien en este último caso se trabajó sobre una muestra de un pavimento algo diferente a los que están en uso actualmente, la coincidencia en la tendencia, indica un nuevo punto a favor de la tecnología led y su vínculo con la eficiencia energética.

\footnotetext{
${ }^{22}$ Ixtaina, P., et al, "Spectral Reflectance of Argentinean Road Surfaces", Proceedings of the Lux Europa 2017, European Lighting Conference, Ljubljana, Slovenia, 2017b, pp. 612-617.
} 
Capítulo 8 - Análisis ulterior y conclusiones 


\section{1.- Marco actual: la iluminación LED}

La luminotecnia viene cursando en los últimos años un cambio de paradigma generado por la irrupción del LED como fuente luminosa. Si bien el descubrimiento de la generación de luz a partir de una corriente en un semiconductor data de mediados del siglo pasado, el desarrollo de dispositivos LED con alta capacidad de emisión, junto con eficiencias comparables y superiores a algunas de las lámparas en uso, ha producido una verdadera revolución en el diseño y construcción de artefactos para iluminación.

El tema de las fuentes luminosas, sus tipos y formas de funcionamiento, no ha sido abordado en esta Tesis, ya que los postulados de las hipótesis presentadas, con la sola excepción de lo expuesto en el capítulo 7, resultan independientes del tipo de emisor empleado. Sin embargo, resulta conveniente enmarcar los resultados obtenidos en este cambio de paradigma.

Lo que se puede considerar hoy como lámparas eléctricas tradicionales, se divide en dos grandes grupos: incandescentes y de descarga. En las primeras, la luz es la parte visible de la radiación térmica de un filamento y su temperatura se eleva por el paso de una corriente eléctrica. En las lámparas de descarga, la luz es producida por la excitación a nivel atómico de un gas o vapor contenido entre dos electrodos (plasma), que genera emisiones radiantes. Las características finales de la luz obtenida dependen del tipo de gas empleado, de la presión a la que se encuentra y de la presencia o no de una película de material fluorescente en la pared interior de la lámpara. ${ }^{1}$

En los LEDs, la luz es emitida directamente a partir de fotones generados en el interior de una juntura semiconductora. Por no ser necesarios mecanismos intermedios (calor o movimientos y excitación de átomos en un plasma), es de esperar una emisión más eficiente que en las lámparas tradicionales. En efecto, leds individuales y en condiciones experimentales han superado los $300 \mathrm{~lm} / \mathrm{W},{ }^{2}$ mientras que las lámparas de descarga más eficientes (sodio baja presión) tienen un límite de $180 \mathrm{~lm} / \mathrm{W}$, alcanzado en la década del ' $50 .{ }^{3}$

La alta eficiencia del LED, promocionada incluso en forma exagerada por motivos comerciales, se reduce considerablemente cuando el dispositivo emisor forma parte de un producto concreto (luminaria). El efecto es más notable en las luminarias para alumbrado público cuyo diseño y tecnología tuvo desde sus orígenes la función central de alumbrar con mínimo consumo de energía. ${ }^{4}$ En este campo, la diferencia no es tan notoria, ${ }^{5}$ sin embargo, factores técnicos y comerciales están extendiendo el uso de equipamiento LED para estos fines. Por un lado, eficacias entre un 30 y un $40 \%$ superiores a las lámparas de sodio alta presión (predominantes en el alumbrado público), con posibilidades ciertas de

\footnotetext{
${ }^{1}$ Illuminating Enineering Society (IES), The Lighting Handbook, 9na. edición, McGraw-Hill, Estados Unidos, 2011; Coaton, J. R., Marsden, A. M., Lamps and lighting, 4ta edición, Wiley, NewYork, 1997; Ixtaina, P., "Lámparas. Apuntes de clase", Cátedra de Dispositivos Eléctricos, Facultad de Ingeniería, Universidad Nacional de La Plata, 2009, mímeo.

${ }^{2}$ Mottier, P., LEDs for Lighting Applications, John Wiley \& Sons, Inc, 2009; Cangeloso, S., LED Lighting, O’Reilly Media, Inc, 2012.

${ }^{3}$ Philips Professional, Product Catalog, [en línea] URL: http://www.lighting.philips.com/main/prof [Consulta: 6 de abril de 2019].

${ }^{4}$ van Bommel, W., Road Lighting - Fundamentals, Technology and Apllication, Springer, 2015.

5 Ixtaina, P., Armas, A., "Iluminación led en autopistas argentinas", Memorias del XIII Congreso Panamericano de Iluminación LUXAMÉRICA 2016, Chile, 2016.
} 
incrementarse en los próximos años; componentes electrónicos con menor tasa de fallas; menor sensibilidad a golpes y vibraciones (frente a la fragilidad propia de las lámparas). Se suma a lo anterior la generación de luz blanca con su contribución a la estética de alumbrado público y un costo de producción con tendencia decreciente, característica propia de un dispositivo totalmente electrónico con componentes previamente desarrollados. Lo anterior permite afirmar que las luminarias LED serán exclusivas en pocos años más, quedando las lámparas tradicionales relegadas a aplicaciones muy particulares.

Los párrafos anteriores hacen referencia al cambio de fuente luminosa, que es lo central del pasaje global a tecnología LED. Aunque resulte obvio, es necesario remarcar que el uso de LEDs no altera las leyes de la luminotecnia, ni los métodos de cálculo y diseño. Conviene aquí resaltar el concepto de reconversión de una instalación de alumbrado, muy en boga actualmente de la mano del pasaje a LED. Por reconversión de una instalación se entiende "su mejora con eje en el aumento de la eficiencia del alumbrado, logrando con ello un ahorro de energía a partir de la incorporación de una nueva tecnología. ${ }^{6} \mathrm{Si}$ bien el término no se empleó siempre, estos procesos no son nuevos: se considera una primera reconversión cuando irrumpe el alumbrado público eléctrico, en la que se emplean lámparas incandescentes para el reemplazo de farolas o mecheros de gas o aceite. Entre las décadas del ' 50 y 60, en las principales capitales de Argentina, se produce la primera reconversión "moderna", con el pasaje a lámparas de descarga en lugar de las fuentes incandescentes. Se emplean lámparas vapor de mercurio alta presión, lo que introduce un cambio en eficacia comparable al actual, con un incremento de casi un $400 \%$ (de $12 \mathrm{~lm} / \mathrm{W}$ a casi $60 \mathrm{~lm} / \mathrm{W}$ ).

Posteriormente, desde fines de los ' 80 , se introduce en el mercado la lámpara de sodio alta presión y se desarrolla una nueva reconversión, con un importante fomento por parte del Estado (plan Luces Bonaerenses, PRONUREE, etc.), logrando que estas lámparas se impongan en el alumbrado público. Mejoras tecnológicas generaron a su vez un aumento paulatino en su eficacia, hasta alcanzar los 120/140 lm/W actuales.

La mayoría de estas reconversiones estuvieron acompañadas por luminarias mejoradas, nuevas ópticas y cuidados diseños de la instalación, orientados a optimizar la eficiencia global del sistema. Paradójicamente, vemos que esta política no es general en las reconversiones leds actuales. En realidad, lo predominante es un recambio de luminarias que muchas veces resulta en instalaciones sobredimensionadas, iluminaciones que no verifican los parámetros de calidad normalizados y que muchas veces no logran ahorros sustanciales de energía. Aquí lo masivo ${ }^{7}$ predomina con su faceta económica por sobre el aspecto técnico, con una importante responsabilidad de los profesionales de la ingeniería que descuidan la parte central de toda reconversión, que es la etapa de diseño.

\footnotetext{
${ }^{6}$ Ixtaina, P., Bannert, B., Bufo, "Eficiencia energética en reconversiones Led del alumbrado público", Memorias de las XIII Jornadas Argentinas de Luminotecnia, Mendoza, 2017c.

${ }^{7}$ Ver, por ejemplo: Cermele, L. "En La Plata se inició el recambio de 14 mil luminarias a luces led", Radio Provincia, 31 de octubre de 2018, [en línea] URL: http://provinciaradio.com.ar/index.php/2018/10/31/enla-plata-se-inicio-el-recambio-de-14-mil-luminarias-a-luces-led/ [Consulta: 3 de abril de 2019]; "Las luminarias Led cubrirán casi el 50\% del alumbrado público de Paraná”, El Diario, 23 de enero de 2019, [en línea] URL: https://www.eldiario.com.ar/18694-las-luminarias-led-cubriran-casi-el-50-del-alumbradopublico-de-parana/ [Consulta: 3 de abril de 2019].
} 
Resulta entonces necesario remarcar que una luminaria LED puede emitir más que una de igual potencia con tecnología de descarga (aspecto que debe ser seriamente verificado) y generar una iluminación "blanca" seguramente más atractiva que el sodio. Pero esto de ninguna manera implica que su reemplazo en una instalación redunde en una reconversión del alumbrado. Por el contario, solo conociendo su "forma" de emisión, la geometría de instalación, el tipo de arteria, etc., es posible realizar un diseño efectivo que permita optimizar su desempeño para ese tipo de calzada. Lo anterior es justamente el diseño de la instalación, acción que debe fomentarse y, en este sentido, la docencia en luminotecnia es de gran importancia.

\section{2.- Producto de la tesis}

La aplicación de la técnica de luminancia está comprendida en la problemática, aunque en este caso, lo delicado de la situación (riesgos vinculados a la circulación vehicular a alta velocidad) impone más cuidados en el momento de la reconversión. Parte de lo producido en la Tesis ya ha sido aplicado en los dos casos de reconversiones de iluminaciones de autopistas ya realizados (y descriptos en los capítulos anteriores): autopistas de la red AUSA y La Plata-Buenos Aires. Está implementación tuvo como eje la hipótesis central: "Las calzadas locales son particulares de esta región y no pueden aplicarse datos (tablas-r) obtenidas de estudios europeos". Sobre este punto remarcamos:

a.- Se demostró, a partir de mediciones de luminancia, que los valores de diseños no se verificaban al momento de evaluar la instalación nueva. La principal discrepancia entre el proyecto y su evaluación, se encontraba en la uniformidad (el valor medido resultó siempre más bajo que el de proyecto). Estos resultados permitieron presuponer que las calzadas locales eran más especulares que las tabla-r estandarizadas.

b.- Se desarrolló un método, que incluyó un equipo de medición, para obtener aproximaciones a la tabla-r real a partir de registros en el lugar. El método y el equipo fueron ajustados y calibrados en laboratorio y posteriormente se efectuaron aplicaciones concretas en autopistas. La técnica propuesta fue validada internacionalmente mediante su presentación en una reunión específica de la Comisión Internacional de Alumbrado CIE, que convocó a los máximos especialista en el tema.

c.- En el marco de una transferencia tecnológica solicitada por concesionarios viales, se realizaron aplicaciones del Reflectómetro de Calzadas en las autopistas de accesos y urbanas de la ciudad de Buenos Aires. Esta investigación permitió generar una base de datos con información real de las propiedades reflectivas de las calzadas de la región.

Los puntos b y c permiten completar la Tesis en el sentido de brindar todos los elementos necesarios para la aplicación de la técnica de luminancia con datos locales, sin necesidad de recurrir a las tablas-r de la estandarización europea. En línea con lo expuesto en el punto 1 , se brindan elementos para realizar diseños de instalaciones viales por luminancia con adecuada exactitud. Este cálculo es independiente del tipo de fuente luminosa y tal como se describió en los capítulos 4 y 5 , fue realizado con buenos resultados para la reconversión a LED de las autopistas de la Red AUSA (2013/14) y la Autopista Buenos Aires La Plata (2014). Es importante remarcar que, en ambos cálculos, los proyectistas emplearon tablas-r de la "clasificación local", específicamente las obtenidas en mediciones en la autopista 25 de Mayo. Posteriormente se implementaron "zonas testigo", instalando las nuevas luminarias en tramos de con entre 4 y 5 columnas 
modificados y sobre estás "áreas de evaluación" se realizaron mediciones de normalizadas de iluminancia y luminancia. Los resultados obtenidos verificaron los datos de proyecto, lo que afirma el trabajo realizado.

Finalmente, en el desarrollo de los capítulos 6 y 7, se brindaron elementos que deben entenderse como base para futuras investigaciones. Por un lado, se verificó la hipótesis que afirmaba la influencia del coeficiente de luminancia medio de la superficie de la calzada en la eficiencia energética de la instalación de alumbrado y se comprobó además que los pavimentos de la zona estudiada poseen discrepancias entre ellos de magnitud importante. Resta analizar cómo su estructura interna (piedra, aglutinante, método de aplicación) se manifiesta en esta "mayor claridad", con la idea de avanzar hacia nuevas autopistas y rutas con pavimentos más eficientes energéticamente. Por otro lado, la influencia del espectro LED avanza en el mismo sentido: cuál es la combinación óptima entre espectro (por ejemplo, cuál es la temperatura de color de la fuente LED) más adecuada para interactuar en forma eficiente con la superficie de la calzada.

El consumo energético del alumbrado público vial de una ciudad ronda (dependiendo del grado de industrialización) entre un 20 y $30 \%$ del total. ${ }^{8}$ También es muy importante la energía consumida por la iluminación de trazas de autopistas. En ambos casos, no es necesario explayarse sobre las ventajas del alumbrado en la vida moderna: seguridad vial, seguridad personal, mejora en la efectividad del transporte, activación económica de zonas comerciales, etc. A la par, la energía se vuelve -y se volverá- un bien escaso que es necesario preservar. Se espera haber producido elementos para contribuir a diseños de futuras iluminaciones con adecuada exactitud, posibilitando así la optimización de los factores que afectan a la eficiencia energética y a la seguridad vial.

\footnotetext{
${ }^{8}$ Ixtaina, P., Vidal, P., "Reconversión Energética en instalaciones de alumbrado público. La experiencia de Olavarría (Argentina)", Anales del 7mo Congreso Panamericano de Iluminación, LUXAMERICA 2004, Lima (Perú), 2004.
} 


\section{Bibliografía}

Adrian, W., Jobanputra, R., "Influence of Pavement Reflectance on Lighting for Parking Lots", Portland and Cement Association, PCA R\&D Serial No 2458, 2005.

Alcalde, H., Bazalar Vidal, P., "Investigación de pavimentos de la provincia de Buenos Aires", Memorias de las V Jornadas Argentinas de Iluminación, Rosario, 1982.

Bardier, D., De la Visión al Conocimiento, Tradinco editores, Montevideo, 2001.

Bazalar, P, Ixtaina, P., "Alumbrado vial en base al criterio de luminancia. Comentario sobre los errores en una instalación de alumbrado público unilateral real", Revista Luminotecnia, 48, 1995.

Bazalar, P., Ixtaina, P., "Seguridad de las personas en rutas de tránsito rápido. Investigaciones en el Laboratorio de la CIC sobre reflectancia lumínica tendientes a mejorar los actuales métodos en uso", Anales del III Congreso Panamericano de Luminotecnia, 1995a.

Blackwell H. R., "Contrast thresholds of the human eye", Journal of the Optical Society of America, 36(11), 1946.

Bodmann, H., Schmidt, H., "Road surface reflection and Road Lighting: Field investigations", Lighting Research and Technology, 21(4), 1989, pp. 159-170.

Burghout, F., "Two class system for the classification of dry road surfaces with regard to light reflection", CIE Journal 5(1), pp. 22-43, 1986.

Cangeloso, S., LED Lighting, O’Reilly Media Inc, Sebastopol, 2012.

Cermele, L. "En La Plata se inició el recambio de 14 mil luminarias a luces led", Radio Provincia, 31 de octubre de 2018, [en línea] URL: http://provinciaradio.com.ar/index.php/2018/10/31/en-la-plata-se-inicio-el-recambio-de-14mil-luminarias-a-luces-led/ [Consulta: 3 de abril de 2019].

CEN/TR 13201-1 Technical Report, Road Lighting - Part1: Selection of lighting classes, Bruselas, 2004.

CIE, Calculation and measurement of luminance and illuminance in road lighting, Publicación 30, 1976.

CIE, Calculation and measurement of luminance and illuminance in road lighting, Publicación 30-2 - TC 4.6, Francia, 1982.

CIE, Discomfort Glare in the Interior Working Environment, publicación 55, Francia, 1983.

CIE, Recommendations for the lighting of roads for motor and pedestrian traffic, publicación 115, TC-4.15, Vienna, 1995.

CIE, Road surface and road marking reflection characteristics, publicación TC 4.24 y 4.25-144, Francia, 2001.

CIE, International Lighting Vocabulary, publicación 017/E ILV, Austria, 2011.

CIE, Appendix 7: Photopic spectral luminous efficiency function, [en línea] URL: https://onlinelibrary.wiley.com/doi/pdf/10.1002/0470024275.app7 [Consulta: 3 de abril de 2019]. Viena, 1931.

Coaton, J. R., Marsden, A. M., Lamps and lighting, 4ta edición, Wiley, NewYork, 1997.

Conti, F., "Fisiología de la Visión", Fisiología Médica (capítulo 18), Mc Graw Hill, C. de México, 2010.

De Boer J., Burghout F., Van Heemskerck Veeckens, J. F. T., "Appraisal of the quality of public lighting based on road surface luminance and glare", 14va Sesión del CIE, Bruselas, P5923, 1959.

De Boer, J., Cohu, M., Schreuder, D., Public Lighting. Philips technical Library, Países Bajos, 1967.

De Boer, J., Fischer, D., Interior Lighting, Philips Technical Library, Deventer, 1978.

De Boer, J., Van Bommel, W., Road Lighting, Philips technical Library, Países Bajos, 1980.

Ekrias, A., Ylinen, A., Eloholma, M., Halonen, L., "Effects of Pavement Lightness and Colour on Road Lighting Performance", CIE International Symposium on road surface photometric characteristics: Measurement Systems and Results, Italia, 2008.

Erbay, A., Atlas of the reflection properties of road surfaces, ILTUB, Berlin, 1974.

Federal Highway Administration, Reflective characteristics of roadway pavements during wet weather, reporte FHWA/RD-86/104, 1986. 
Herold, M. Remote Sensing of Impervious Surfaces, Ch. 12 - Spectral Characteristics of Asphalt Road Surfaces, Wageningen University, The Netherlands, 2007.

Holladay, L., "The fundamentals of glare and visibility", Journal of the Optical Society of America, 12(4), 1926.

Hopkinson, R. G., Discomfort glare in lighted streets, Transactions of the IES, 5(1), Londres, 1940.

Illuminating Enineering Society (IES), The Lighting Handbook, 9na. edición, McGraw-Hill, N. Y., 2011.

Instituto Argentino de Racionalización de Materiales, IRAM AADL J 2022-2, Alumbrado Público, Vias de Tránsito - Clasificación y Niveles de Iluminación, Buenos Aires, 1995.

Instituto Argentino de Racionalización de Materiales, IRAM AADL J 2022-2, Alumbrado Público, Vias de Tránsito - Clasificación y niveles de iluminación, Buenos Aires, 2009.

International Organization for Standardization ISO, "Guide to the expression of uncertainty in measurement", Suiza, 1993.

ISO 23539, 2005 (E)/CIE S 010, Photometry - The CIE system of physical photometry, 2004.

ISO/CIE 19476, 2014 (E), Characterization of the Performance of Illuminance Meters and Luminance Meters, 2014.

Ixtaina, P., "Lámparas. Apuntes de clase", Cátedra de Dispositivos Eléctricos, Facultad de Ingeniería, Universidad Nacional de La Plata, 2009, mímeo.

Ixtaina, P., Informe Cientifico del Investigador 2013/14, Repositorio digital CIC [en línea] URL: http://digital.cic.gba.gob.ar/handle/11746/1869 [Consulta: 3 de abril de 2019].

Ixtaina, P., Informe Científico del Investigador 2015/16, Repositorio digital CIC, [en línea] URL: http://digital.cic.gba.gob.ar/handle/11746/6450 [Consulta: 3 de abril de 2019].

Ixtaina, P., Vidal, P., "Reconversión Energética en instalaciones de alumbrado público. La experiencia de Olavarría (Argentina)", Anales del $7 m o$ Congreso Panamericano de Iluminación, LUXAMERICA 2004, Lima (Perú), 2004.

Ixtaina, P. R., Vidal, P. A., "Changes with use and age of reflection properties of road surface new types", CIE Midterm Meeting and International Lighting Congress, León, 2005.

Ixtaina P., Vidal P., "Argentinean road surface characterization", International CIE Symposium on road surface photometric characteristics: Measurement Systems and Results, Torino, Italia, 2008.

Ixtaina, P., Presso, M. "Estudio de Calzadas en las Autopistas Urbanas de la ciudad de Buenos Aires", Actas del X Congreso Panamericano de Iluminación, Valparaíso (Chile), Octubre de 2010, pp. 108-113.

Ixtaina, P., Vidal, P., Ferreyra, J. "Eficiencia energética en instalaciones de alumbrado vial". Memorias del XI Congreso Iberoamericano de Iluminación "LUXAMÉRICA 2012", Cartagena (Colombia), octubre de 2012, pp. 159-163.

Ixtaina, P., Vidal, P., "Road surface lightness and energy efficiency in road lighting", Proceedings of the 12th European Lighting Conference "LUX EUROPA 2013", Cracovia (Polonia), 2013.

Ixtaina, P., Armas, A., Bannert, B., "Use effects on the reflection of macro textured surfaces", Journal of Applied Engineering Sciences, 6(19), 2016a, pp. 51-56.

Ixtaina, P., Armas, A., "Iluminación led en autopistas argentinas", Memorias del XIII Congreso Panamericano de Iluminación LUXAMÉRICA 2016, Chile, 2016.

Ixtaina, P., et al, "Argentinean Experience in Highways Led Lighting”, Energy and Environment Research, 7(1), 2017, pp. 31-37.

Ixtaina, P. et al, "Effects of led lighting on the luminance coefficient", Light \& Engineering, 25(4), 2017a, pp. 115-122.

Ixtaina, P., et al, "Spectral Reflectance of Argentinean Road Surfaces", Proceedings of the Lux Europa 2017, European Lighting Conference, Ljubljana, Slovenia, 2017b, pp. 612-617.

Ixtaina, P., Bannert, B., Bufo, N. "Eficiencia energética en reconversiones Led del alumbrado público", Memorias de las XIII Jornadas Argentinas de Luminotecnia, Mendoza, 2017c.

Keitz, H. A. E, Light calculations and measurements, N. V. Philips, Holanda, 1974. 
"Las luminarias Led cubrirán casi el 50\% del alumbrado público de Paraná", El Diario, 23 de enero de 2019, [en línea] URL: https://www.eldiario.com.ar/18694-las-luminarias-ledcubriran-casi-el-50-del-alumbrado-publico-de-parana/ [Consulta: 3 de abril de 2019].

Laurent, M., "Characterisation of Road Surfaces using Memphis mobile gonio reflectometer", CIE International Symposium on road surface photometric characteristics: Measurement Systems and Results, Italia, 2008.

Moon, P., The Scientific Basis of Illuminating Engineering, McGraw Hill Book Company, Londres, 1936.

Mottier, P., LEDs for Lighting Applications, John Wiley \& Sons, Inc, N.J., 2009.

Muzet, V., "Coluroute: a mobile goniophotomer to characterize the road surface photometry", CIE International Symposium on road surface photometric characteristics: Measurement Systems and Results, Italia, 2008.

Narváez, A., "La microscopía", Nociones básicas de óptica (capítulo 2), [en línea] URL: http://www.medic.ula.ve/histologia/anexos/microscopweb/MONOWEB/inicio.htm [Consulta: 9/4/19].

Pracki, P., "A proposal to classify road lighting energy efficiency", Lighting Research and Technology, 43, 2011, pp. 271-280.

Range, H., "Road Reflectometer for the simplified determination of the properties of lighted road surfaces", Lichttechnik, 25, p. 389, 1973.

Rossi, G., "Synthesis of the discussions on "photometric measurements of Road surfaces", $C I E$ symposium on road surface photometric characteristics - Measurement systems and results. Turin, 2008.

Sørensen K., Road Surface reflection data. Lysteknisk Laboratorium, The Danish illuminating Engineering Laboratory. Lyngby, Dinamarca, Reporte $N^{\circ} 10,1975$.

Superintendencia de Riesgos del Trabajo. Higiene y seguridad en el trabajo. Protocolo para la Medición de la Iluminación en el Ambiente Laboral. Resolución 84/2012. Ministerio de Justicia y Derechos Humanos. Presidencia de la Nación [en línea] URL: http://servicios.infoleg.gob.ar/infolegInternet/anexos/190000-194999/193616/norma.htm [Consulta: 2 de abril de 2019].

Tomsak, R., Levine, M., Handbook of neuro-ophtalmology, Butterworth-Heinemann, Oxford, 2003.

van Bommel, W., de Boer, J., Road Lighting, Philips Technical Library, Países Bajos, 1982.

van Bommel, W., Road Lighting - Fundamentals, Technology and Apllication, Springer, Nuenen, 2015.

Vidal, P. A., Ixtaina, P. R., "New System of identification of de reflection indicatrices of pavements", note to CIE TC 4-25, Francia, 1996.

Vidal, P. A., Ixtaina, P. R., "Application of a New Pavement Reflectometer", 2nd Balkan Conference and Fair in Lighting Balkan-Light'02, Proceedings, Estambul, 2002.

Vidal, P. A., Ixtaina, P. R., "New method for characterising the reflection properties of road surfaces", CIE 25 ${ }^{\text {th }}$ Session, San Diego, 2003.

Walsh, J., Photometry, Constable \& Company Ltd., Londres, 1958.

Wordenweber, B., Boyce, P., et al, Automotive Lighting and Human Vision, Springer, 2007.

Ziegler, W., Reflexiontrockener und feuchterStrassenbelage Dissertation, Universitat Karlsruhe, Karlsruhe, 1980.

\section{Sitios web}

Commission Internationale de l'Eclairage (CIE), [en línea] URL: http://www.cie.co.at [Consulta: 2 de abril de 2019].

"Company", Photo Research. A Line of Light \& Color Measurement Soutions, [en línea] URL: http://www.photoresearch.com/content/company [Consulta: 2 de abril de 2019].

CIE, "Division 4 Technical Committees", Commission Internationale de l'Eclairage (CIE), [en línea] URL: http://cie.co.at/technical-work/division4/technical-committees [Consulta: 2 de abril de 2019]. 
Everfine [en línea] URL: http://www.everfine.net/ [Consulta: 2 de abril de 2019].

"Informes Científicos del Investigador", Repositorio Digital CIC, [en línea] URL: https://digital.cic.gba.gob.ar/discover?query=informes + cient $\% \mathrm{C} 3 \% \mathrm{ADficos} \& 1 \mathrm{r}=$ [Consulta: 2 de abril de 2019].

"Instruments for Photometry", LMT Lichtmesstechnik GMB, [en línea] URL: http://www.lmtberlin.de/en/ophoto.html [Consulta: 2 de abril de 2019].

Laboratorio de Acústica y Luminotecnia - Comisión de investigaciones Científicas de la provincia de Buenos Aires, [en línea] URL: http://lal.cic.gba.gob.ar/ [Consulta: 2 de abril de 2019].

Laboratorio de Entrenamiento Multidisciplinario para la Investigación Tecnológica (LEMIT), [en línea] URL: http://www.lemit.gov.ar/ [Consulta: 2 de abril de 2019].

"Lux meter HT3424", HIOKI, [en línea], URL: https://www.hioki.com/en/products/detail/?product key $=5659$ [Consulta: 2 de abril de 2019].

"Luminance Meter LMT 1009", LMT Lichtmesstechnik GMB, [en línea] URL: http://www.lmtberlin.de/en/11009.html [Consulta: 3 de abril de 2019].

"Memorias del LAL. Memorias CIC PBA", Repositorio Digital CIC, [en línea] URL: https://digital.cic.gba.gob.ar/handle/11746/1812 [Consulta: 2 de abril de 2019].

Philips Professional, Product Catalog, [en línea] URL: http://www.lighting.philips.com/main/prof [Consulta: 6 de abril de 2019].

"Photometer Heads", LMT Lichtmesstechnik GMB, [en línea] URL: http://www.lmtberlin.de/en/pheads.html [Consulta: 2 de abril de 2019].

"Photometric Sensor Catalog", Gamma Scientific, [en línea] URL: http://www.gammasci.com/products/photometric-sensors/ [Consulta: 2 de abril de 2019].

"StarLine", Avantes. Enlightening Spectroscopy, [en línea] URL: http://www.avantes.com/products/spectrometers/starline [Consulta: 3 de abril de 2019].

"Subsecretaría de Ahorro y Eficiencia Energética", Plan Alumbrado Eficinete PLAE URL: https://www.argentina.gob.ar/energia/ahorro-y-eficiencia-energetica/edificaciones-sectorpublico/alumbrado-publico-eficiente\#3 [Consulta: 10 de abril de 2019]. 
Anexo I - Capítulo 4. Características reflectivas de las tablas-r
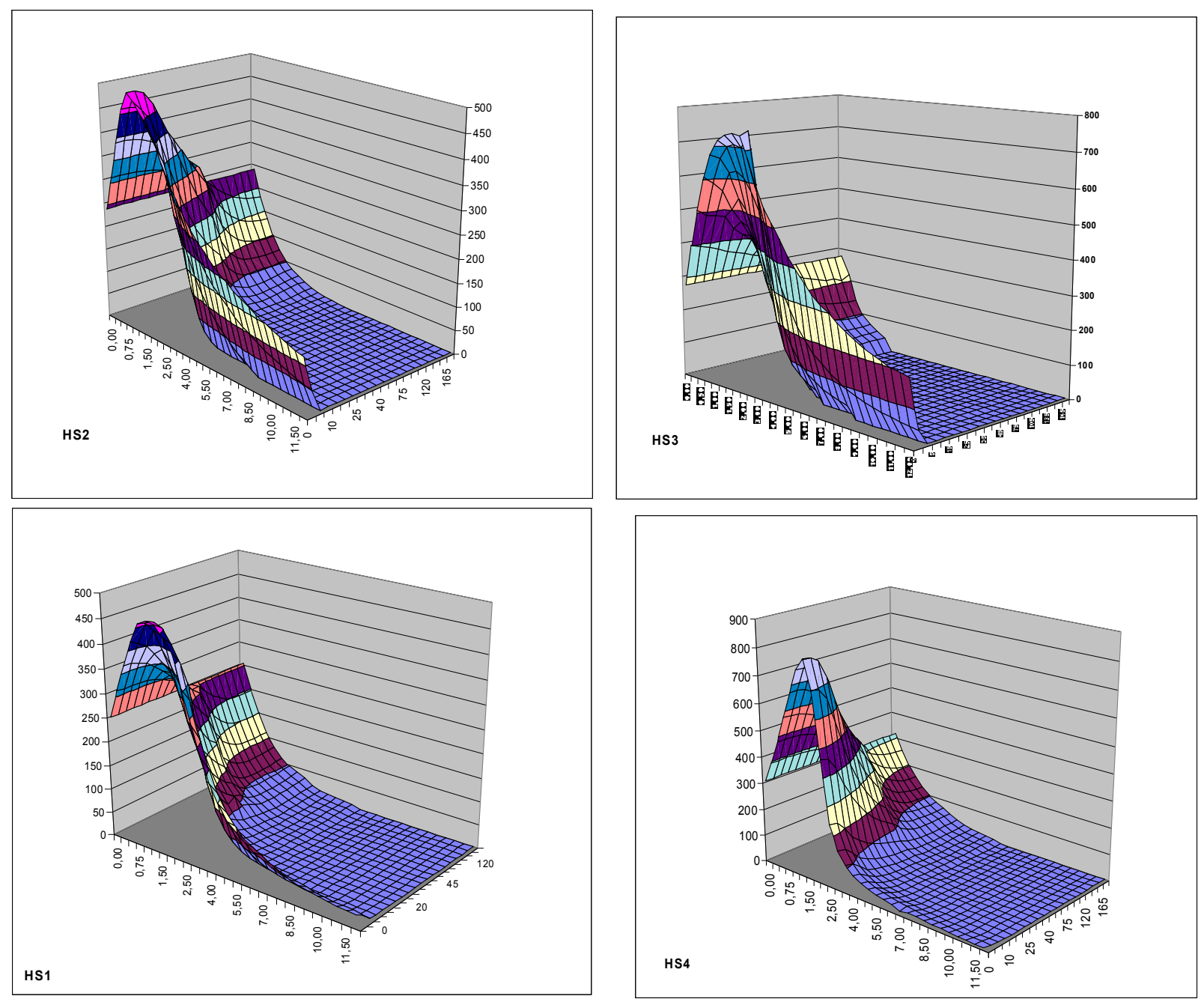

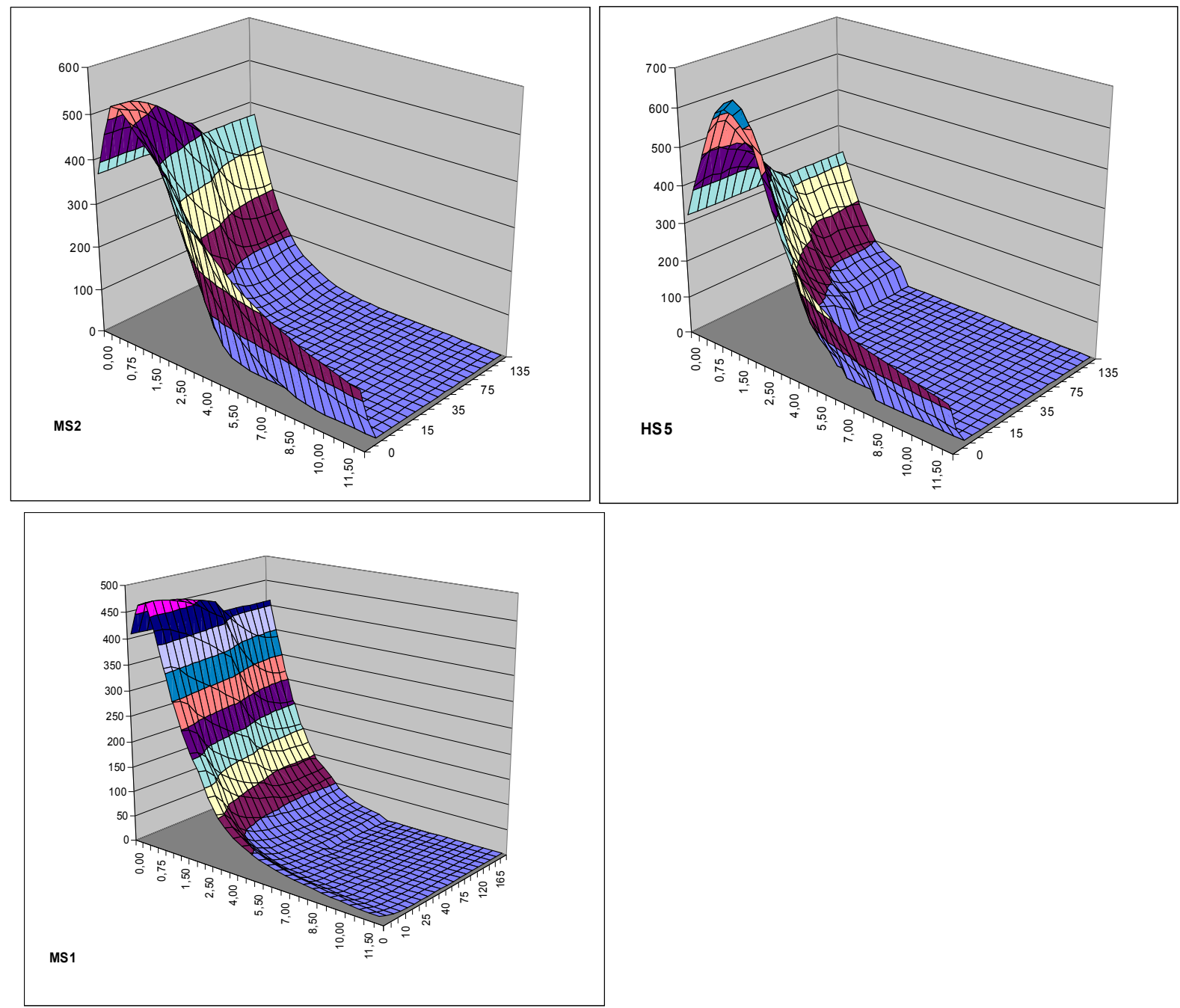